\title{
Xpert MTB/RIF Ultra and Xpert MTB/RIF assays for extrapulmonary tuberculosis and rifampicin resistance in adults (Review)
}

Kohli M, Schiller I, Dendukuri N, Yao M, Dheda K, Denkinger CM, Schumacher SG, Steingart KR

Kohli M, Schiller I, Dendukuri N, Yao M, Dheda K, Denkinger CM, Schumacher SG, Steingart KR.

Xpert MTB/RIF Ultra and Xpert MTB/RIF assays for extrapulmonary tuberculosis and rifampicin resistance in adults.

Cochrane Database of Systematic Reviews 2021, Issue 1. Art. No.: CD012768.

DOI: 10.1002/14651858.CD012768.pub3.

www.cochranelibrary.com

Xpert MTB/RIF Ultra and Xpert MTB/RIF assays for extrapulmonary tuberculosis and rifampicin resistance in adults (Review) 
HEADER

ABSTRACT

PLAIN LANGUAGE SUMMARY

SUMMARY OF FINDINGS

BACKGROUND

Figure 1.

OBJECTIVES

METHODS

RESULTS

Figure 2.

Figure 3.

Figure 4.

Figure 5.

Figure 6.

Figure 7.

Figure 8.

Figure 9.

Figure 10.

Figure 11.

Figure 12.

Figure 13.

Figure 14.

Figure 15.

Figure 16.

Figure 17.

DISCUSSION

AUTHORS' CONCLUSIONS

ACKNOWLEDGEMENTS

REFERENCES

CHARACTERISTICS OF STUDIES

ADDITIONAL TABLES

WHAT'S NEW

HISTORY

CONTRIBUTIONS OF AUTHORS

DECLARATIONS OF INTEREST

SOURCES OF SUPPORT

DIFFERENCES BETWEEN PROTOCOL AND REVIEW

INDEX TERMS

\section{TABLE OF CONTENTS}




\title{
Xpert MTB/RIF Ultra and Xpert MTB/RIF assays for extrapulmonary tuberculosis and rifampicin resistance in adults
}

\author{
Mikashmi Kohli ${ }^{1}$ Ian Schiller², Nandini Dendukuri², Mandy Yao², Keertan Dheda ${ }^{3,4}$, Claudia M Denkinger ${ }^{5,6}$, Samuel G Schumacher ${ }^{5}$, \\ Karen R Steingart ${ }^{7}$ \\ ${ }^{1}$ Department of Epidemiology, Biostatistics and Occupational Health, McGill University, Montreal, Canada. ${ }^{2}$ Centre for Outcomes \\ Research, McGill University Health Centre - Research Institute, Montreal, Canada. ${ }^{3}$ Centre for Lung Infection and Immunity Unit, \\ Department of Medicine and UCT Lung Institute, University of Cape Town, Cape Town, South Africa. ${ }^{4}$ Faculty of Infectious and Tropical \\ Diseases, Department of Immunology and Infection, London School of Hygiene and Tropical Medicine, London, UK. ${ }^{5}$ FIND, Geneva, \\ Switzerland. ${ }^{6}$ Division of Tropical Medicine, Centre for Infectious Diseases, University Hospital Heidelberg, Heidelberg, Germany. \\ ${ }^{7}$ Honorary Research Fellow, Department of Clinical Sciences, Liverpool School of Tropical Medicine, Liverpool, UK
}

Contact address: Karen R Steingart, karen.steingart@gmail.com.

Editorial group: Cochrane Infectious Diseases Group.

Publication status and date: New search for studies and content updated (no change to conclusions), published in Issue 1, 2021.

Citation: Kohli M, Schiller I, Dendukuri N, Yao M, Dheda K, Denkinger CM, Schumacher SG, Steingart KR. Xpert MTB/RIF Ultra and Xpert MTB/RIF assays for extrapulmonary tuberculosis and rifampicin resistance in adults. Cochrane Database of Systematic Reviews 2021, Issue 1. Art. No.: CD012768. DOI: 10.1002/14651858.CD012768.pub3.

Copyright (c) 2021 The Authors. Cochrane Database of Systematic Reviews published by John Wiley \& Sons, Ltd. on behalf of The Cochrane Collaboration. This is an open access article under the terms of the Creative Commons Attribution-Non-Commercial Licence, which permits use, distribution and reproduction in any medium, provided the original work is properly cited and is not used for commercial purposes.

\section{A B S T R A C T}

\section{Background}

Xpert MTB/RIF Ultra (Xpert Ultra) and Xpert MTB/RIF are World Health Organization (WHO)-recommended rapid nucleic acid amplification tests (NAATs) widely used for simultaneous detection of Mycobacterium tuberculosis complex and rifampicin resistance in sputum. To extend our previous review on extrapulmonary tuberculosis (Kohli 2018), we performed this update to inform updated WHO policy (WHO Consolidated Guidelines (Module 3) 2020).

\section{Objectives}

To estimate diagnostic accuracy of Xpert Ultra and Xpert MTB/RIF for extrapulmonary tuberculosis and rifampicin resistance in adults with presumptive extrapulmonary tuberculosis.

\section{Search methods}

Cochrane Infectious Diseases Group Specialized Register, MEDLINE, Embase, Science Citation Index, Web of Science, Latin American Caribbean Health Sciences Literature, Scopus, ClinicalTrials.gov, the WHO International Clinical Trials Registry Platform, the International Standard Randomized Controlled Trial Number Registry, and ProQuest, 2 August 2019 and 28 January 2020 (Xpert Ultra studies), without language restriction.

\section{Selection criteria}

Cross-sectional and cohort studies using non-respiratory specimens. Forms of extrapulmonary tuberculosis: tuberculous meningitis and pleural, lymph node, bone or joint, genitourinary, peritoneal, pericardial, disseminated tuberculosis. Reference standards were culture and a study-defined composite reference standard (tuberculosis detection); phenotypic drug susceptibility testing and line probe assays (rifampicin resistance detection). 


\section{Data collection and analysis}

Two review authors independently extracted data and assessed risk of bias and applicability using QUADAS-2. For tuberculosis detection, we performed separate analyses by specimen type and reference standard using the bivariate model to estimate pooled sensitivity and specificity with $95 \%$ credible intervals (Crls). We applied a latent class meta-analysis model to three forms of extrapulmonary tuberculosis. We assessed certainty of evidence using GRADE.

\section{Main results}

69 studies: 67 evaluated Xpert MTB/RIF and 11 evaluated Xpert Ultra, of which nine evaluated both tests. Most studies were conducted in China, India, South Africa, and Uganda. Overall, risk of bias was low for patient selection, index test, and flow and timing domains, and low $(49 \%)$ or unclear (43\%) for the reference standard domain. Applicability for the patient selection domain was unclear for most studies because we were unsure of the clinical settings.

\section{Cerebrospinal fluid}

Xpert Ultra (6 studies)

Xpert Ultra pooled sensitivity and specificity (95\% Crl) against culture were $89.4 \%$ (79.1 to 95.6) (89 participants; low-certainty evidence) and $91.2 \%$ (83.2 to 95.7) (386 participants; moderate-certainty evidence). Of 1000 people where 100 have tuberculous meningitis, 168 would be Xpert Ultra-positive: of these, 79 (47\%) would not have tuberculosis (false-positives) and 832 would be Xpert Ultra-negative: of these, $11(1 \%)$ would have tuberculosis (false-negatives).

\section{Xpert MTB/RIF (30 studies)}

Xpert MTB/RIF pooled sensitivity and specificity against culture were $71.1 \%$ (62.8 to 79.1 ) (571 participants; moderate-certainty evidence) and $96.9 \%$ (95.4 to 98.0) (2824 participants; high-certainty evidence). Of 1000 people where 100 have tuberculous meningitis, 99 would be Xpert MTB/RIF-positive: of these, 28 (28\%) would not have tuberculosis; and 901 would be Xpert MTB/RIF-negative: of these, 29 (3\%) would have tuberculosis.

\section{Pleural fluid}

Xpert Ultra (4 studies)

Xpert Ultra pooled sensitivity and specificity against culture were $75.0 \%$ (58.0 to 86.4 ) (158 participants; very low-certainty evidence) and $87.0 \%$ (63.1 to 97.9) (240 participants; very low-certainty evidence). Of 1000 people where 100 have pleural tuberculosis, 192 would be Xpert Ultra-positive: of these, $117(61 \%)$ would not have tuberculosis; and 808 would be Xpert Ultra-negative: of these, 25 (3\%) would have tuberculosis.

\section{Xpert MTB/RIF (25 studies)}

Xpert MTB/RIF pooled sensitivity and specificity against culture were $49.5 \%$ (39.8 to 59.9) (644 participants; low-certainty evidence) and 98.9\% (97.6 to 99.7) (2421 participants; high-certainty evidence). Of 1000 people where 100 have pleural tuberculosis, 60 would be Xpert MTB/RIF-positive: of these, 10 (17\%) would not have tuberculosis; and 940 would be Xpert MTB/RIF-negative: of these, 50 (5\%) would have tuberculosis.

\section{Lymph node aspirate}

Xpert Ultra (1 study)

Xpert Ultra sensitivity and specificity (95\% confidence interval) against composite reference standard were $70 \%$ (51 to 85 ) (30 participants; very low-certainty evidence) and 100\% (92 to 100) (43 participants; low-certainty evidence). Of 1000 people where 100 have lymph node tuberculosis, 70 would be Xpert Ultra-positive and 0 (0\%) would not have tuberculosis; 930 would be Xpert Ultra-negative and 30 (3\%) would have tuberculosis.

\section{Xpert MTB/RIF (4 studies)}

Xpert MTB/RIF pooled sensitivity and specificity against composite reference standard were $81.6 \%$ (61.9 to 93.3) (377 participants; lowcertainty evidence) and $96.4 \%$ (91.3 to 98.6) (302 participants; low-certainty evidence). Of 1000 people where 100 have lymph node tuberculosis, 118 would be Xpert MTB/RIF-positive and 37 (31\%) would not have tuberculosis; 882 would be Xpert MTB/RIF-negative and $19(2 \%)$ would have tuberculosis.

In lymph node aspirate, Xpert MTB/RIF pooled specificity against culture was $86.2 \%$ ( 78.0 to 92.3 ), lower than that against a composite reference standard. Using the latent class model, Xpert MTB/RIF pooled specificity was $99.5 \%$ (99.1 to 99.7 ), similar to that observed with a composite reference standard. 


\section{Rifampicin resistance}

Xpert Ultra (4 studies)

Xpert Ultra pooled sensitivity and specificity were $100.0 \%$ (95.1 to 100.0), (24 participants; low-certainty evidence) and $100.0 \%$ (99.0 to 100.0) (105 participants; moderate-certainty evidence). Of 1000 people where 100 have rifampicin resistance, 100 would be Xpert Ultrapositive (resistant): of these, zero ( $0 \%$ ) would not have rifampicin resistance; and 900 would be Xpert Ultra-negative (susceptible): of these, zero $(0 \%)$ would have rifampicin resistance.

\section{Xpert MTB/RIF (19 studies)}

Xpert MTB/RIF pooled sensitivity and specificity were $96.5 \%$ (91.9 to 98.8 ) (148 participants; high-certainty evidence) and $99.1 \%$ ( 98.0 to 99.7) (822 participants; high-certainty evidence). Of 1000 people where 100 have rifampicin resistance, 105 would be Xpert MTB/RIF-positive (resistant): of these, 8 ( $8 \%$ ) would not have rifampicin resistance; and 895 would be Xpert MTB/RIF-negative (susceptible): of these, 3 (0.3\%) would have rifampicin resistance.

\section{Authors' conclusions}

Xpert Ultra and Xpert MTB/RIF may be helpful in diagnosing extrapulmonary tuberculosis. Sensitivity varies across different extrapulmonary specimens: while for most specimens specificity is high, the tests rarely yield a positive result for people without tuberculosis. For tuberculous meningitis, Xpert Ultra had higher sensitivity and lower specificity than Xpert MTB/RIF against culture. Xpert Ultra and Xpert MTB/RIF had similar sensitivity and specificity for rifampicin resistance. Future research should acknowledge the concern associated with culture as a reference standard in paucibacillary specimens and consider ways to address this limitation.

\section{PLAIN LANGUAGE SUMMARY}

How accurate are tests (Xpert Ultra and Xpert MTB/RIF) for diagnosing tuberculosis outside the lungs (extrapulmonary tuberculosis) and rifampicin resistance?

\section{Why is using Xpert tests for extrapulmonary tuberculosis important?}

Tuberculosis is one of the top 10 causes of death worldwide. Tuberculosis mainly affects the lungs (pulmonary) but may occur in other parts of the body (extrapulmonary). When people receive proper and timely treatment, tuberculosis is usually curable. One problem involved in managing tuberculosis is that the bacteria become resistant to antibiotics. Not recognizing tuberculosis early may result in delayed diagnosis and treatment and increased illness and death. An incorrect tuberculosis diagnosis may result in increased anxiety and unnecessary treatment.

\section{What is the aim of this review?}

To update the evidence on accuracy of Xpert tests for diagnosing extrapulmonary tuberculosis and rifampicin resistance in adults. Rifampicin is an important tuberculosis drug. We included tuberculous meningitis and pleural, lymph node, bone or joint, genitourinary, peritoneal, pericardial, and disseminated tuberculosis.

\section{What was studied in this review?}

Xpert Ultra and Xpert MTB/RIF are rapid tests for simultaneously diagnosing tuberculosis and rifampicin resistance. We combined study results to determine:

- sensitivity: people with tuberculosis (rifampicin resistance) correctly diagnosed as having the condition.

- specificity: people without tuberculosis (rifampicin resistance) correctly identified as not having the condition.

The closer sensitivity and specificity are to $100 \%$, the better the test. We measured Xpert results against culture and a composite reference standard (neither is a perfect reference standard because extrapulmonary tuberculosis is paucibacillary (few bacteria)).

\section{What are the main results in this review?}

69 studies tested lymph node, pleural, and cerebrospinal fluid, and other specimens from people with presumptive extrapulmonary tuberculosis. Studies were conducted in 28 different countries.

For every 1000 people tested, if 100 had tuberculosis according to the reference standards:

\section{cerebrospinal fluid}

-Xpert Ultra (6 studies):

. $89 \%$ sensitivity: 168 people would test positive, including 79 without tuberculosis

. 91\% specificity: 832 people would test negative, including 11 with tuberculosis 
- Xpert MTB/RIF (30 studies):

. $71 \%$ sensitivity: 99 people would test positive, including 28 without tuberculosis

. 97\% specificity: 901 people would test negative, including 29 with tuberculosis

\section{pleural fluid}

- Xpert Ultra (4 studies):

- $75 \%$ sensitivity: 192 people would test positive, including 117 without tuberculosis

. $87 \%$ specificity: 808 people would test negative, including 25 with tuberculosis

- Xpert MTB/RIF (25 studies):

. $50 \%$ sensitivity: 60 people would test positive, including 10 without tuberculosis

. 99\% specificity: 940 would test negative, including 50 with tuberculosis

\section{lymph node fluid}

- Xpert Ultra (1 study):

- $70 \%$ sensitivity: 70 people would test positive (all have tuberculosis)

- 100\% specificity: 930 people would test negative, including 30 with tuberculosis

-Xpert MTB/RIF (4 studies):

- $82 \%$ sensitivity:118 people would test positive, including 37 without tuberculosis

-96\% specificity: 882 people would test negative, including 19 with tuberculosis

\section{rifampicin resistance}

-Xpert Ultra (4 studies):

-100\% sensitivity: 100 people would test positive (all have rifampicin resistance)

- 100\% specificity: 900 people would test negative (none have rifampicin resistance)

- MTB/RIF test (19 studies):

- 97\% sensitivity: 105 people would test positive, including eight without rifampicin resistance

. 99\% specificity: 895 people would test negative, including three with rifampicin resistance

\section{Who do the results of this review apply to?}

People thought to have extrapulmonary tuberculosis.

\section{How confident are we in our results?}

Fairly confident for Xpert MTB/RIF in cerebrospinal fluid and less so in lymph node fluid. Less confident for Xpert Ultra, as there were few studies and few people tested. Both reference standards are imperfect, which may affect accuracy estimates.

\section{What are the implications of this review?}

The Xpert tests may be helpful in diagnosing extrapulmonary tuberculosis. Sensitivity varies across different extrapulmonary specimens, while for most specimens, specificity is high, the test rarely yielding a positive result for people without tuberculosis (verified by culture). For tuberculous meningitis, Xpert Ultra had higher sensitivity than Xpert MTB/RIF and lower specificity than Xpert MTB/RIF. The tests had similar accuracy for diagnosing rifampicin resistance.

\section{How up-to-date is this review?}

28 January 2020. 


\section{SUMMARY OF FINDINGS}

\section{Summary of findings 1. Xpert Ultra and Xpert MTB/RIF in cerebrospinal fluid}

Participants: people presumed to have tuberculous meningitis

Prior testing: people who received Xpert Ultra or Xpert MTB/RIF testing may first have undergone a health examination (history and physical examination) and possibly received a chest radiograph

Role: initial test, replacement for usual practice

Settings: primarily tertiary care centres (the index test was often run in reference laboratories)

Index tests: Xpert Ultra and Xpert MTB/RIF

Reference standard: solid or liquid culture

Studies: cross-sectional studies

Limitations: participants were evaluated exclusively as inpatients at a tertiary care centre, or, if the clinical setting was not reported, Xpert was performed at a reference laboratory rather than at primary care facilities and local hospitals

Xpert Ultra pooled sensitivity ( $95 \% \mathrm{Crl}$ ): $89.4 \%$ (79.1 to 95.6 ); pooled specificity (95\% CrI): $91.2 \%$ ( 83.2 to 95.7 )

Xpert MTB/RIF pooled sensitivity (95\% CrI): $71.1 \%$ (62.8 to 79.1); pooled specificity (95\% CrI): $96.9 \%$ (95.4 to 98.0)

\begin{tabular}{|c|c|c|c|c|c|}
\hline \multirow[t]{2}{*}{ Xpert Ultra result } & \multicolumn{3}{|c|}{1000 people tested for TB using Xpert Ultra(95\% CrI) } & \multirow{2}{*}{$\begin{array}{l}\text { Number } \\
\text { of par- } \\
\text { ticipants } \\
\text { (studies) }\end{array}$} & \multirow{2}{*}{$\begin{array}{l}\text { Certain- } \\
\text { ty of the } \\
\text { evidence } \\
\text { (GRADE) }\end{array}$} \\
\hline & Prevalence of $2.5 \%$ & Prevalence of $10 \%$ & Prevalence of $20 \%$ & & \\
\hline \multirow[t]{2}{*}{ True-positives (participants with TB meningitis) } & 22 & 89 & 178 & $89(6)$ & $\oplus \oplus \ominus \ominus$ \\
\hline & (20 to 24$)$ & (79 to 96$)$ & (158 to 191$)$ & & Low $^{a}$ \\
\hline \multirow{2}{*}{$\begin{array}{l}\text { False-negatives (participants incorrectly classified as not having } \\
\text { TB meningitis) }\end{array}$} & 3 & 11 & 22 & & \\
\hline & (1 to 5$)$ & (4 to 21 ) & (9 to 42 ) & & \\
\hline \multirow[t]{2}{*}{ True-negatives (participants without TB meningitis) } & 889 & 821 & 730 & $386(6)$ & $\oplus \oplus \oplus \ominus$ \\
\hline & (811 to 933 ) & (749 to 861 ) & (666 to 766$)$ & & Moderateb \\
\hline \multirow{2}{*}{$\begin{array}{l}\text { False-positives (participants incorrectly classified as having TB } \\
\text { meningitis) }\end{array}$} & 86 & 79 & 70 & & \\
\hline & (42 to 164 ) & (39 to 151 ) & (34 to 134$)$ & & \\
\hline
\end{tabular}




\begin{tabular}{|c|c|c|c|c|c|c|}
\hline \multirow{2}{*}{\multicolumn{2}{|c|}{ 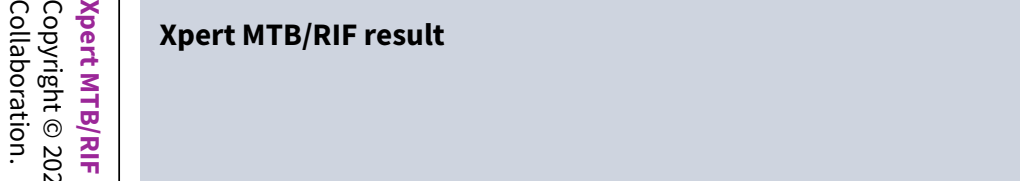 }} & \multicolumn{3}{|c|}{1000 people tested for TB using XpertMTB/RIF ( $95 \% \mathrm{CrI}$ ) } & \multirow{2}{*}{$\begin{array}{l}\text { Number } \\
\text { of par- } \\
\text { ticipants } \\
\text { (studies) }\end{array}$} & \multirow{2}{*}{$\begin{array}{l}\text { Certain- } \\
\text { ty of the } \\
\text { evidence } \\
\text { (GRADE) }\end{array}$} \\
\hline & & Prevalence of $2.5 \%$ & Prevalence of $10 \%$ & Prevalence of $20 \%$ & & \\
\hline 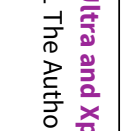 & True-positives (participants with TB meningitis) & $\begin{array}{l}18 \\
\text { (16 to } 20)\end{array}$ & $\begin{array}{l}71 \\
\text { (63 to 79) }\end{array}$ & $\begin{array}{l}142 \\
\text { (126 to } 158)\end{array}$ & $571(30)$ & $\begin{array}{l}\oplus \oplus \oplus \odot \\
\text { Moderate }\end{array}$ \\
\hline 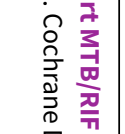 & $\begin{array}{l}\text { False-negatives (participants incorrectly classified as not having } \\
\text { TB meningitis) }\end{array}$ & $\begin{array}{l}7 \\
\text { (5 to 9) }\end{array}$ & $\begin{array}{l}29 \\
\text { (21 to } 37 \text { ) }\end{array}$ & $\begin{array}{l}58 \\
\text { (42 to } 74 \text { ) }\end{array}$ & & \\
\hline 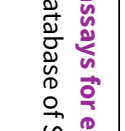 & True-negatives (participants without TB meningitis) & $\begin{array}{l}945 \\
\text { (930 to 956) }\end{array}$ & $\begin{array}{l}872 \\
\text { (859 to 882) }\end{array}$ & $\begin{array}{l}775 \\
\text { (763 to } 784 \text { ) }\end{array}$ & $2824(30)$ & $\begin{array}{l}\oplus \oplus \oplus \oplus \\
\text { High }\end{array}$ \\
\hline 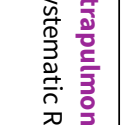 & $\begin{array}{l}\text { False-positives (participants incorrectly classified as having TB } \\
\text { meningitis) }\end{array}$ & $\begin{array}{l}30 \\
\text { (19 to } 45)\end{array}$ & $\begin{array}{l}28 \\
\text { (18 to } 41)\end{array}$ & $\begin{array}{l}25 \\
\text { (16 to } 37 \text { ) }\end{array}$ & & \\
\hline
\end{tabular}

Abbreviations: Crl: credible interval; TB: tuberculosis

We included plausible prevalence estimates for the target condition suggested by the WHO. For Xpert Ultra, the median prevalence of tuberculosis in the included studies was $35.2 \%$. For Xpert MTB/RIF, the median prevalence of tuberculosis in the included studies was $15.2 \%$.

Credible limits were estimated based on those around the point estimates for pooled sensitivity and specificity.

$a$ There were few participants in this analysis. The very wide $95 \% \mathrm{Crl}$ around true-positives and false-negatives may lead to different decisions, depending on which credible limits are assumed. We downgraded two levels for imprecision.

bThe wide $95 \% \mathrm{Crl}$ around true-negatives and false-positives would likely lead to different decisions, depending on which credible limits are assumed. We downgraded one level for imprecision.

cThe wide $95 \% \mathrm{Crl}$ around true-positives and false-negatives may lead to different decisions, depending on which credible limits are assumed. We downgraded one level for imprecision.

\section{GRADE certainty of the evidence}

High: we are very confident that the true effect lies close to that of the estimate of the effect.

Moderate: we are moderately confident in the effect estimate: the true effect is likely to be close to the estimate of the effect, but there is a possibility that it is substantially different.

Low: our confidence in the effect estimate is limited: the true effect may be substantially different from the estimate of the effect.

Very low: we have very little confidence in the effect estimate: the true effect is likely to be substantially different from the estimate of effect.

The results presented in this table should not be interpreted in isolation from results of the individual included studies contributing to each summary test accuracy measure.

\section{Summary of findings 2. Xpert Ultra and Xpert MTB/RIF in pleural fluid}

Participants: people presumed to have pleural tuberculosis 
Prior testing: people who received Xpert Ultra or Xpert MTB/RIF testing may first have undergone a health examination (history and physical examination) and received a chest radiograph

Role: initial test, replacement for usual practice, which may include more invasive tests, such as pleural biopsy

Settings: primarily tertiary care centres (the index test was often run in reference laboratories)

Index tests: Xpert Ultra and Xpert MTB/RIF

Reference standard: solid or liquid culture

Studies: cross-sectional studies

Limitations: in most studies, participants were evaluated at a tertiary care centre, or if the clinical setting was not reported, the test was performed at a reference laborato-

Xpert Ultra pooled sensitivity ( $95 \% \mathrm{Crl}$ ): $\mathbf{7 5 . 0} \%$ ( 58.0 to 86.4 ); pooled specificity (95\% CrI): $87.0 \%$ (63.1 to 97.9 )

Xpert MTB/RIF pooled sensitivity (95\% CrI): $49.5 \%$ (39.8 to 59.9); pooled specificity (95\% CrI): $98.9 \%$ (97.6 to 99.7)

\begin{tabular}{|c|c|c|c|c|c|}
\hline \multirow[t]{2}{*}{ Xpert Ultra result } & \multicolumn{3}{|c|}{1000 people tested for TB using Xpert Ultra $(95 \% \mathrm{Crl})$} & \multirow{2}{*}{$\begin{array}{l}\text { Number } \\
\text { of par- } \\
\text { ticipants } \\
\text { (studies) }\end{array}$} & \multirow{2}{*}{$\begin{array}{l}\text { Certainty of } \\
\text { the evidence } \\
\text { (GRADE) }\end{array}$} \\
\hline & Prevalence of $2.5 \%$ & Prevalence of $\mathbf{1 0 \%}$ & Prevalence of $\mathbf{2 0 \%}$ & & \\
\hline True-positives (patients with pleural TB) & $\begin{array}{l}19 \\
(14 \text { to } 22)\end{array}$ & $\begin{array}{l}75 \\
(58 \text { to } 86)\end{array}$ & $\begin{array}{l}150 \\
(116 \text { to } 173)\end{array}$ & \multirow[t]{2}{*}{$158(4)$} & $\begin{array}{l}\oplus \odot \odot \ominus \\
\text { Very lowa,b,c }\end{array}$ \\
\hline $\begin{array}{l}\text { False-negatives (patients incorrectly classified as not having } \\
\text { pleural TB) }\end{array}$ & $\begin{array}{l}6 \\
\text { (3 to } 11)\end{array}$ & $\begin{array}{l}25 \\
(14 \text { to } 42)\end{array}$ & $\begin{array}{l}50 \\
(27 \text { to } 84)\end{array}$ & & \\
\hline True-negatives (patients without pleural TB) & $\begin{array}{l}848 \\
(615 \text { to } 955)\end{array}$ & $\begin{array}{l}783 \\
\text { (568 to } 881)\end{array}$ & $\begin{array}{l}696 \\
(505 \text { to } 783)\end{array}$ & \multirow[t]{2}{*}{$240(4)$} & $\begin{array}{l}\oplus \Theta \Theta \Theta \\
\text { Very lowa,d,e }\end{array}$ \\
\hline $\begin{array}{l}\text { False-positives (patients incorrectly classified as having pleur- } \\
\text { al TB) }\end{array}$ & $\begin{array}{l}127 \\
(20 \text { to } 360)\end{array}$ & $\begin{array}{l}117 \\
\text { (19 to } 332)\end{array}$ & $\begin{array}{l}104 \\
\text { (17 to } 295)\end{array}$ & & \\
\hline \multirow[t]{2}{*}{ Xpert MTB/RIF result } & \multicolumn{3}{|c|}{1000 people tested for TB using Xpert MTB/RIF (95\% CrI) } & Number & Certainty of \\
\hline & Prevalence of $2.5 \%$ & Prevalence of $\mathbf{1 0} \%$ & Prevalence of $\mathbf{2 0 \%}$ & $\begin{array}{l}\text { ticipants } \\
\text { (studies) }\end{array}$ & (GRADE) \\
\hline True-positives (patients with pleural TB) & 12 & 50 & 99 & $644(25)$ & $\begin{array}{l}\oplus \oplus \odot \odot \\
\text { Lowf,g,h }\end{array}$ \\
\hline
\end{tabular}




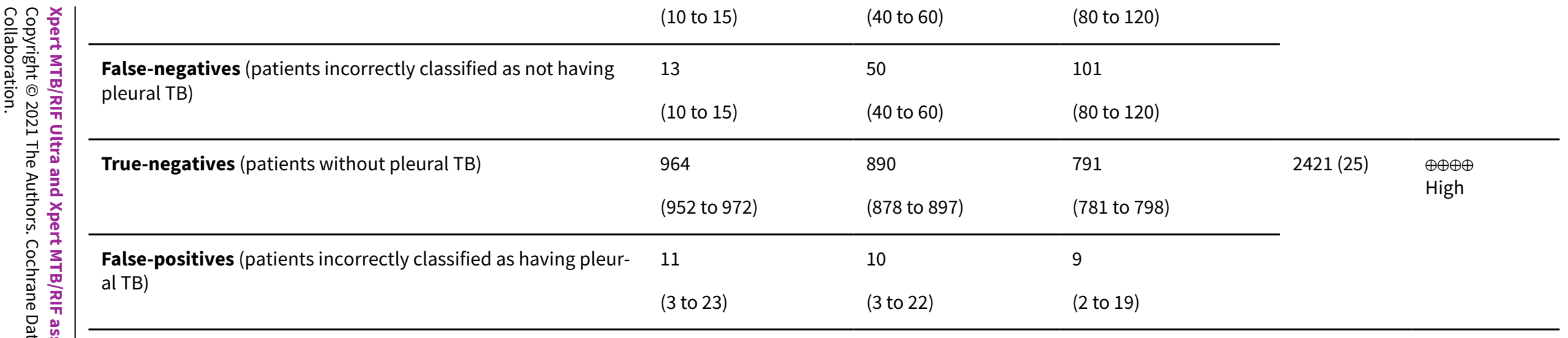

\section{Abbreviations: Crl: credible interval; TB: tuberculosis.}

We included plausible prevalence estimates for the target condition suggested by the WHO. For Xpert Ultra, the median prevalence of tuberculosis in the included studies was $46.2 \%$. For Xpert MTB/RIF, the median prevalence of tuberculosis in the included studies was $19.8 \%$.

aWe were interested in how Xpert Ultra performed in patients presumed to have extrapulmonary tuberculosis who were evaluated as they would be in routine practice. However, most studies did not report information on the clinical setting. We downgraded one level for indirectness.

bFor individual studies, sensitivity estimates ranged from $48 \%$ to $84 \%$. We could not explain the heterogeneity by study quality or other factors. We downgraded one level for inconsistency.

cThere was a low number of participants contributing to this analysis for the observed sensitivity. As we had already downgraded for inconsistency, we downgraded one level for imprecision.

dFor individual studies, specificity estimates ranged from $65 \%$ to $100 \%$. We could not explain the heterogeneity by study quality or other factors. We downgraded one level for inconsistency.

eWe thought the wide $95 \% \mathrm{Crl}$ around false-positives and true-negatives would likely lead to different decisions depending on which confidence limits are assumed. As we had already downgraded for inconsistency, we downgraded one level for imprecision.

fWe were interested in how Xpert MTB/RIF performed in participants presumed to have extrapulmonary tuberculosis who were evaluated as they would be in routine practice. However, most studies did not report information on the clinical setting. We downgraded one level for indirectness.

gFor individual studies, sensitivity estimates ranged from $10 \%$ to $100 \%$. We could not explain the heterogeneity by study quality or other factors. We downgraded one level for inconsistency.

hAs we had already downgraded for inconsistency, we did not downgrade further for imprecision.

\section{GRADE certainty of the evidence}

High: we are very confident that the true effect lies close to that of the estimate of the effect.

Moderate: we are moderately confident in the effect estimate: the true effect is likely to be close to the estimate of the effect, but there is a possibility that it is substantially different.

Low: our confidence in the effect estimate is limited: the true effect may be substantially different from the estimate of the effect.

Very low: we have very little confidence in the effect estimate: the true effect is likely to be substantially different from the estimate of effect.

The results presented in this table should not be interpreted in isolation from results of the individual included studies contributing to each summary test accuracy measure.

\section{Summary of findings 3. Xpert Ultra and Xpert MTB/RIF in lymph node aspirate}

Participants: people presumed to have lymph node tuberculosis 
Prior testing: people who received Xpert testing may first have undergone a health examination (history and physical examination) and possibly received a chest radiograph

Role: initial test, replacement for usual practice, which may include more invasive tests, such as biopsy of affected organs

Settings: primarily tertiary care centres (the index test was often run in reference laboratories)

Index tests: Xpert Ultra and Xpert MTB/RIF

Reference standard: composite reference standard

Studies: cross-sectional studies

Limitations: in most studies, participants were evaluated at a tertiary care centre, or if the clinical setting was not reported, the test was performed at a reference laboratory performed at a reference laboratory

Xpert Ultra sensitivity (95\% Cl): $70 \%$ (51 to 85 ); specificity: $100 \%$ (92 to 100 )

Xpert MTB/RIF pooled sensitivity (95\% CrI): $81.6 \%$ (61.9 to 93.3 ); pooled specificity: $96.4 \%$ (91.3 to 98.6$)$

\begin{tabular}{|c|c|c|c|c|c|}
\hline \multirow[t]{2}{*}{ Xpert Ultra result } & \multicolumn{3}{|c|}{$\begin{array}{l}1000 \text { people tested for TB using Xpert Ultra } \\
(95 \% \mathrm{Cl})\end{array}$} & \multirow{2}{*}{$\begin{array}{l}\text { Number } \\
\text { of par- } \\
\text { ticipants } \\
\text { (studies) }\end{array}$} & \multirow{2}{*}{$\begin{array}{l}\text { Certainty of } \\
\text { the evidence } \\
\text { (GRADE) }\end{array}$} \\
\hline & Prevalence of $2.5 \%$ & Prevalence of $10 \%$ & Prevalence of $\mathbf{2 0 \%}$ & & \\
\hline True-positives (patients with lymph node TB) & 17 (13 to 21$)$ & 70 (51 to 85$)$ & $140(102$ to 170$)$ & \multirow[t]{2}{*}{$30(1)$} & \multirow{2}{*}{$\begin{array}{l}\oplus \odot \odot \ominus \\
\text { Very lowa,b }\end{array}$} \\
\hline $\begin{array}{l}\text { False-negatives (patients incorrectly classified as not having } \\
\text { lymph node TB) }\end{array}$ & $8(4$ to 12$)$ & 30 (15 to 49$)$ & 60 (30 to 98$)$ & & \\
\hline True-negatives (patients without lymph node TB) & 975 (897 to 975$)$ & $900(828$ to 900$)$ & 800 (736 to 800$)$ & \multirow[t]{2}{*}{$43(1)$} & \multirow{2}{*}{$\begin{array}{l}\oplus \ominus \ominus \ominus \\
\text { Very lowa,c }\end{array}$} \\
\hline $\begin{array}{l}\text { False-positives (patients incorrectly classified as having lymph } \\
\text { node TB) }\end{array}$ & 0 (0 to 78$)$ & 0 (0 to 72$)$ & 0 (0 to 64$)$ & & \\
\hline \multirow[t]{2}{*}{ Xpert MTB/RIF result } & \multicolumn{3}{|c|}{1000 people tested for TB using Xpert MTB/RIF ( $95 \% \mathrm{Crl}$ ) } & \multirow{2}{*}{$\begin{array}{l}\text { Number } \\
\text { of par- } \\
\text { ticipants } \\
\text { (studies) }\end{array}$} & \multirow{2}{*}{$\begin{array}{l}\text { Certainty of } \\
\text { the evidence } \\
\text { (GRADE) }\end{array}$} \\
\hline & Prevalence of $2.5 \%$ & Prevalence of $10 \%$ & Prevalence of $\mathbf{2 0 \%}$ & & \\
\hline True-positives (patients with lymph node TB) & 20 (16 to 23 ) & $81(62$ to 92$)$ & $162(124$ to 184$)$ & \multirow[t]{2}{*}{$377(4)$} & \multirow{2}{*}{$\begin{array}{l}\oplus \oplus \ominus \ominus \\
\text { Lowd,e }\end{array}$} \\
\hline $\begin{array}{l}\text { False-negatives (patients incorrectly classified as not having } \\
\text { lymph node TB) }\end{array}$ & 5 (2 to 9$)$ & 19 (8 to 38$)$ & $38(16$ to 76$)$ & & \\
\hline True-negatives (patients without lymph node TB) & 935 (878 to 958$)$ & 863 (811 to 885$)$ & 767 (721 to 786$)$ & $302(4)$ & $\begin{array}{l}\oplus \oplus \ominus \ominus \\
\text { Lowd,e }\end{array}$ \\
\hline
\end{tabular}


Abbreviations: Crl: credible interval; TB: tuberculosis.

We included plausible prevalence estimates for the target condition suggested by the WHO. For Xpert Ultra, the prevalence of tuberculosis in the included study was $41 \%$. For Xpert MTB/RIF, the median prevalence of tuberculosis in the included studies was $55.5 \%$.

aWe identified only one study, which was conducted at a tertiary referral centre in South Africa, a high TB burden country. Most participants (84\%) were seen as outpatients. With only one study, applicability to other settings comes with some uncertainty. We downgraded one level for indirectness.

bThere were very few participants contributing to this analysis. The $95 \% \mathrm{Cl}$ was very wide. We downgraded two levels for imprecision.

CThere were very few participants contributing to this analysis. The $95 \% \mathrm{Cl}$ was wide. We downgraded two levels for imprecision.

dThe composite reference standard was defined by the primary study authors and therefore, was not uniform. We downgraded one level for risk of bias.

eFor indirectness, regarding applicability, for the patient population, we considered most studies to have unclear concern. We were interested in how Xpert MTB/RIF performed in patients presumed to have extrapulmonary TB who were evaluated as they would be in routine practice. However, none of the studies reported this information. We downgraded one level for indirectness.

\section{GRADE certainty of the evidence}

High: we are very confident that the true effect lies close to that of the estimate of the effect.

Moderate: we are moderately confident in the effect estimate: the true effect is likely to be close to the estimate of the effect, but there is a possibility that it is substantially different.

Low: our confidence in the effect estimate is limited: the true effect may be substantially different from the estimate of the effect.

Very low: we have very little confidence in the effect estimate: the true effect is likely to be substantially different from the estimate of effect.

The results presented in this table should not be interpreted in isolation from results of the individual included studies contributing to each summary test accuracy measure.

\section{Summary of findings 4. Xpert Ultra and Xpert MTB/RIF for rifampicin resistance}

Participants: people with tuberculosis detected by Xpert Ultra or Xpert MTB/RIF

Role: initial test, replacement test for standard practice, which includes culture-based drug susceptibility testing or line probe assay

Settings: primarily tertiary care centres, the index test was often run in central (reference laboratories), where drug susceptibility testing for the reference standard could be performed

Index tests: Xpert Ultra and Xpert MTB/RIF

Reference standard: culture-based drug susceptibility testing using solid or liquid media or line probe assay

Studies: cross-sectional studies

Xpert Ultra pooled sensitivity (95\% CrI): $100.0 \%$ (95.1 to 100.0); pooled specificity (95\% CrI): $100.0 \%$ (99.0 to 100.0)

Xpert MTB/RIF pooled sensitivity (95\% CrI): $96.5 \%$ (91.9 to 98.8 ); pooled specificity (95\% CrI): $99.1 \%$ (98.0 to 99.7) 


\begin{tabular}{|c|c|c|c|c|c|}
\hline & Prevalence of $2 \%$ & Prevalence of $10 \%$ & Prevalence of $15 \%$ & & \\
\hline True-positives (patients correctly classified as rifampicin resistant) & 20 (19 to 20$)$ & $100(95$ to 100$)$ & $150(143$ to 150$)$ & \multirow[t]{2}{*}{$24(4)$} & \multirow{2}{*}{$\begin{array}{l}\oplus \oplus \oplus \ominus \\
\text { Lowa,b }^{a}\end{array}$} \\
\hline $\begin{array}{l}\text { False-negatives (patients incorrectly classified as rifampicin suscepti- } \\
\text { ble) }\end{array}$ & $0(0$ to 1$)$ & $0(0$ to 5$)$ & $0(0$ to 7$)$ & & \\
\hline $\begin{array}{l}\text { True-negatives (patients correctly classified as rifampicin suscepti- } \\
\text { ble) }\end{array}$ & 980 (979 to 980$)$ & $900(899$ to 900$)$ & $850(849$ to 850$)$ & \multirow[t]{2}{*}{$105(4)$} & \multirow{2}{*}{$\begin{array}{l}\oplus \oplus \oplus \odot \\
\text { Moderate }^{a}\end{array}$} \\
\hline False-positives (patients incorrectly classified as rifampicin resistant) & $0(0$ to 1$)$ & $0(0$ to 1$)$ & $0(0$ to 1$)$ & & \\
\hline \multirow[t]{2}{*}{ Xpert MTB/RIF result } & \multicolumn{3}{|c|}{$\begin{array}{l}1000 \text { people tested for rifampicin resistance using Xpert MTB/ } \\
\text { RIF ( } 95 \% \text { Crl) }\end{array}$} & \multirow{2}{*}{$\begin{array}{l}\text { Number } \\
\text { of par- } \\
\text { ticipants } \\
\text { (studies) }\end{array}$} & \multirow{2}{*}{$\begin{array}{l}\text { Certain- } \\
\text { ty of the } \\
\text { evidence } \\
\text { (GRADE) }\end{array}$} \\
\hline & Prevalence of $2 \%$ & Prevalence of $10 \%$ & Prevalence of $15 \%$ & & \\
\hline True-positives (patients correctly classified as rifampicin resistant) & $19(18$ to 20$)$ & 97 (92 to 99) & 145 (138 to 148$)$ & \multirow[t]{2}{*}{$148(19)$} & \multirow{2}{*}{$\begin{array}{l}\oplus \oplus \oplus \oplus \\
\mathrm{High}\end{array}$} \\
\hline $\begin{array}{l}\text { False-negatives (patients incorrectly classified as rifampicin suscepti- } \\
\text { ble) }\end{array}$ & 1 (0 to 2$)$ & $3(1$ to 8$)$ & $5(2$ to 12$)$ & & \\
\hline $\begin{array}{l}\text { True-negatives (patients correctly classified as rifampicin suscepti- } \\
\text { ble) }\end{array}$ & 971 (960 to 977$)$ & $892(882$ to 897$)$ & 842 (833 to 847$)$ & \multirow[t]{2}{*}{$822(19)$} & \multirow[t]{2}{*}{$\begin{array}{l}\oplus \oplus \oplus \oplus \\
\text { High }\end{array}$} \\
\hline False-positives (patients incorrectly classified as rifampicin resistant) & $9(3$ to 20$)$ & $8(3$ to 18$)$ & 8 (3 to 17$)$ & & \\
\hline
\end{tabular}

\section{Abbreviations: Crl: credible interval; TB: tuberculosis.}

We included plausible prevalence estimates for the target condition suggested by the WHO. For Xpert Ultra, the median prevalence of rifampicin resistance in the included studies was $19.2 \%$. For Xpert MTB/RIF, the median prevalence of rifampicin resistance in the included studies was $11.9 \%$.

${ }^{a}$ All these studies were conducted in China (high TB-burden country). Applicability to other settings comes with some uncertainty and therefore we downgraded one level for indirectness.

bThere was a low number of participants contributing to this analysis for the observed sensitivity. We downgraded one level for imprecision.

\section{GRADE certainty of the evidence}

High: we are very confident that the true effect lies close to that of the estimate of the effect.

Moderate: we are moderately confident in the effect estimate: the true effect is likely to be close to the estimate of the effect, but there is a possibility that it is substantially different.

Low: our confidence in the effect estimate is limited: the true effect may be substantially different from the estimate of the effect.

Very low: we have very little confidence in the effect estimate: the true effect is likely to be substantially different from the estimate of effect.

The results presented in this table should not be interpreted in isolation from results of the individual included studies contributing to each summary test accuracy measure. 


\section{B A C K G R O U N D}

Tuberculosis (TB) causes tremendous suffering worldwide and has surpassed HIV/AIDS as the world's leading infectious cause of death. The World Health Organization (WHO) estimates that globally in 2019, 10.0 million (range, 8.9 to 11.0 million) people fell ill with tuberculosis. In 2019, around 1.2 million HIV-negative people died from tuberculosis and 208,000 HIV-positive people died from tuberculosis (WHO Global TB Report 2020). When people receive proper treatment, tuberculosis is treatable and curable. The WHO estimates that from 2000 to 2019 more than 60 million lives were saved by diagnosing and treating tuberculosis. However, the COVID-19 pandemic threatens the gains made over recent years. A modelling study by the WHO suggests that there could be between 200,000 and 400,000 additional tuberculosis deaths in 2020 if, over a period of three months, $25 \%$ to $50 \%$ fewer people were detected and treated with tuberculosis (WHO Global TB Report 2020).

Of the 7.1 million new cases of tuberculosis notified to the WHO in $2019,16 \%$ were cases of extrapulmonary tuberculosis, (range, $8 \%$ in the WHO Western Pacific Region to $24 \%$ in the WHO Eastern Mediterranean Region) (WHO Global TB Report 2020). Among countries in the European Union, extrapulmonary tuberculosis was responsible for $19 \%$ of all notified cases (range, $6 \%$ to $44 \%$ ) (Sandgren 2013). A large retrospective analysis from China found that of 19,279 hospitalised tuberculosis patients, around $33 \%$ had extrapulmonary tuberculosis (Pang 2019). The number of people affected by extrapulmonary tuberculosis is likely to be higher, given that, according to the WHO, extrapulmonary tuberculosis is notified as pulmonary tuberculosis when the two forms exist together, and diagnosing extrapulmonary tuberculosis is challenging, as described below. Additionally, extrapulmonary tuberculosis accounts for an increasing proportion of tuberculosis cases in some countries, in part because of host and genetic considerations, and the association of extrapulmonary tuberculosis and HIV (Golden 2005; Pai 2016; Perkins 2007; Webster 2014). Based on surveillance and epidemiological data, extrapulmonary tuberculosis affects a greater proportion of children than adults (Nelson 2004).

Drug-resistant tuberculosis is a serious threat to global health. For the purpose of surveillance and treatment, drug-resistant tuberculosis is classified as rifampicin-resistant tuberculosis, multidrug-resistant tuberculosis (MDR-TB), and extensively drugresistant tuberculosis. MDR-TB is defined as resistance to at least isoniazid and rifampicin, the two most important first-line anti-tuberculosis drugs. Extensively drug-resistant tuberculosis is defined as MDR-TB plus resistance to at least one drug in the following two classes of medicines used in treatment of MDR-TB: fluoroquinolones and second-line injectable agents. In 2019, there were approximately half a million new cases of rifampicin-resistant tuberculosis (of which $78 \%$ had MDR-TB), with India (27\%), China $(14 \%)$ and the Russian Federation (9\%) accounting for the largest burden (WHO Global TB Report 2020). In 2019, 12,350 cases of extensively drug-resistant tuberculosis were reported (WHO Global TB Report 2020).

In 2014, the World Health Assembly unanimously approved the WHO End TB Strategy, a 20-year strategy devised to end the global tuberculosis epidemic (WHO END TB 2014). Early diagnosis of tuberculosis, including universal drug susceptibility testing (DST) and systematic screening of contacts and high-risk groups, is a part of pillar one of the strategy.

\section{Target condition being diagnosed}

\section{Extrapulmonary TB}

Tuberculosis is caused by infection with Mycobacterium tuberculosis (M tuberculosis) bacteria. Tuberculosis predominantly affects the lungs (pulmonary tuberculosis). Extrapulmonary tuberculosis refers to tuberculosis in parts of the body other than the lungs. Extrapulmonary tuberculosis is known to affect virtually every part of the body, with lymph nodes and the pleura being the most common sites (Sharma 2004). Although active pulmonary tuberculosis is transmissible by droplets spread by coughing, extrapulmonary tuberculosis is thought to result from hematogenous spread (spread by way of the bloodstream) from an initial lung infection and is not infectious. Extrapulmonary tuberculosis can occur alone or together with pulmonary tuberculosis.

The various forms of extrapulmonary tuberculosis cause signs and symptoms related to the structures affected. Table 1 describes the forms of extrapulmonary tuberculosis included in this review, as well as the respective specimens that may be collected for diagnosis.

Diagnosis of extrapulmonary tuberculosis is challenging for several reasons. Many forms of extrapulmonary tuberculosis require invasive diagnostic sampling; gathering adequate specimens can pose risk of harm to the patient and can be costly. Most forms of extrapulmonary tuberculosis are paucibacillary (tuberculosis disease caused by a small number of bacteria), making diagnosis by various tests less sensitive. Culture, for example, has reduced sensitivity in paucibacillary disease. In addition, culture takes several weeks for results and requires a highly-equipped laboratory. Limitations are also associated with histology, which relies on highly-trained operators, and characteristic morphology is shared with other diseases. As a result of these difficulties, diagnosis of extrapulmonary tuberculosis is often made on the grounds of clinical suspicion alone, and many people receive the wrong diagnosis, leading to unnecessary tuberculosis treatment or poor outcomes from untreated extrapulmonary tuberculosis.

Tuberculosis treatment regimens must contain multiple drugs to which the organisms are sensitive to cure tuberculosis and avoid selection for drug resistance. WHO tuberculosis treatment guidelines recommend the same drug regimens for extrapulmonary and pulmonary disease, with notable mention of tuberculous meningitis and bone or joint tuberculosis, for which longer treatment regimens are recommended (WHO 2010; WHO 2017; WHO Compendium 2018). For patients with tuberculous meningitis or tuberculous pericarditis, the use of adjuvant corticosteroid therapy is recommended in addition to appropriate tuberculosis treatment regimens (WHO 2017; WHO Compendium 2018). Other tuberculosis treatment guidelines include Sharma 2017b (India), and those issued by the American Thoracic Society, the Centers for Disease Control and Prevention (CDC), and the Infectious Diseases Society of America (Nahid 2016). The drugs used to treat MDR-TB are less potent and more toxic than the drugs used to treat drug-susceptible tuberculosis, historically requiring two years or more of therapy. However, in December 2019, based on new evidence on the management of drug-resistant tuberculosis, the WHO issued recommendations that all patients with MDR-TB or rifampicin-resistant tuberculosis, including those who are also 
resistant to fluoroquinolones, may benefit from effective all-oral treatment regimens, either shorter or longer (WHO Consolidated Guidelines (Module 4) 2020).

\section{Rifampicin resistance}

Rifampicin inhibits bacterial DNA-dependent RNA polymerase, encoded by the RNA polymerase gene (rpoB) (Hartmann 1967). Resistance to this drug has been associated mainly with mutations in a limited region of the rpoB gene (Telenti 1993). Rifampicin resistance may occur alone or in association with resistance to isoniazid and other drugs. In settings with a high burden of MDR-TB, the presence of rifampicin resistance alone may serve as a proxy for MDR-TB (WHO 2011). People with drug-resistant tuberculosis can transmit the infection to others.

\section{Index test(s)}

The index tests, Xpert MTB/RIF and Ultra Xpert MTB/RIF (Xpert Ultra, the newest version) (Cepheid Inc, Sunnyvale, USA), are nucleic acid amplification tests (NAATs) (i.e. molecular tests) used for diagnosing tuberculosis and rifampicin-resistant tuberculosis. Xpert MTB/RIF and Xpert Ultra cartridges are used with the GeneXpert system (Cepheid 2018; Cepheid 2019). Xpert MTB/RIF and Xpert Ultra are able to detect both $M$ tuberculosis complex and rifampicin resistance within two hours after starting the test, with minimal hands-on technical time. Unlike conventional NAATs, with Xpert MTB/RIF and Xpert Ultra, sample processing and polymerase chain reaction (PCR) amplification and detection are integrated into a single, self-enclosed test unit, the GeneXpert cartridge. Following sample loading, all steps in the assay are completely automated and self-contained. In addition, the assays' sample reagent, used to liquefy sputum, has potent tuberculocidal (the ability to kill tuberculosis bacteria) properties and so largely eliminates biosafety concerns during the test procedure (Banada 2010). Except as described below for Ultra trace call results, a single Xpert MTB/RIF or Xpert Ultra run will provide both detection of tuberculosis and detection of rifampicin resistance. One cannot deselect testing for rifampicin resistance and only run the assay for tuberculosis detection.

The development of Xpert MTB/RIF was a major step toward improving detection of tuberculosis and rifampicin resistance globally (Boehme 2010; Small 2011). Since Xpert MTB/RIF was released, there have been four generations ( $G 1, G 2, G 3$, and G4) of the test involving different software and cartridge combinations. Although in comparison with smear microscopy Xpert MTB/RIF has increased sensitivity for pulmonary tuberculosis (Steingart 2014), the test has suboptimal sensitivity in people with smearnegative and HIV-associated tuberculosis. A Cochrane Review on the diagnostic accuracy of Xpert MTB/RIF for pulmonary tuberculosis found pooled sensitivity and specificity (95\% credible Interval (CrI)) of $85 \%(82 \%$ to $88 \%)$ and $98 \%$ (97\% to $98 \%)$, (70 studies, 37,237 unselected participants; high-certainty evidence) (Horne 2019). However, Xpert MTB/RIF sensitivity was decreased in people with smear-negative culture-positive disease, pooled sensitivity of $67 \%$ (62\% to $72 \%$ ), and people living with HIV, pooled sensitivity of $81 \%$ ( $75 \%$ to $86 \%$ ) (Horne 2019). Xpert MTB/RIF versions have also had some limitations in detecting rifampicin resistance.

In order to overcome limitations with Xpert MTB/RIF, Cepheid developed Xpert Ultra, a re-engineered assay that uses a newly- developed cartridge but may be run on the same device after a software upgrade. To improve sensitivity for tuberculosis detection, Xpert Ultra incorporates two different multi-copy amplification targets and a larger DNA reaction chamber than Xpert MTB/RIF (WHO Xpert Ultra 2017). A laboratory study reported that the limit of detection (the lowest number of colony-forming units (CFUs) per sample that can be reproducibly distinguished from negative samples with 95\% confidence) using Xpert Ultra improved to 15.6 $\mathrm{CFU} / \mathrm{mL}$ of sputum compared to $112.6 \mathrm{CFU} / \mathrm{mL}$ for Xpert MTB/RIF (Chakravorty 2017). Xpert Ultra has added a new result category, 'trace call', that corresponds to the lowest bacillary load for $M$ tuberculosis detection (WHO Xpert Ultra 2017). This new category is reported as 'MTB trace DETECTED'. Interpreting a trace call result requires a reassessment of clinical symptoms and history of prior tuberculosis. No rifampicin resistance results are available (indeterminate) for people with trace results. As with Xpert MTB/RIF (Miotto 2012), Xpert Ultra detects both live and dead bacteria.

To address limitations in rifampicin resistance detection, Xpert Ultra uses melting temperature-based analysis, in lieu of realtime PCR analysis with Xpert MTB/RIF. Melting temperaturebased analysis allows Xpert Ultra to better distinguish resistanceconferring mutations from silent mutations with improved diagnostic accuracy for rifampicin resistance detection (Global Laboratory Initiative 2017).

For sputum specimens, the test procedure may be used either directly on raw sputum specimens or sputum pellets created after decontaminating and concentrating the sputum (Blakemore 2010). In both cases, the test material is combined with the assay sample reagent (sodium hydroxide and isopropanol), mixed by hand or vortex, and incubated at room temperature for 15 minutes. After the incubation step, $2 \mathrm{~mL}$ of the treated specimen are transferred to the cartridge and the run is initiated (Helb 2010). According to the manufacturer, as with Xpert MTB/RIF, Xpert Ultra may be used with fresh sputum specimens, which may be either unprocessed sputum or processed sputum sediments. The sample reagent:sample volume ratio is 2:1 for unprocessed sputum and 3:1 for sputum pellets. The manufacturer does not specifically mention the use of the index tests with frozen specimens (Cepheid 2018; Cepheid 2019). As with Xpert MTB/RIF, Xpert Ultra using the GeneXpert sytem requires an uninterrupted and stable electrical power supply, temperature control, and yearly calibration of the cartridge modules (Global Laboratory Initiative 2019). Like previous Xpert cartridge generations, Xpert Ultra can be performed by operators with minimal technical expertise (Theron 2014a). The time to run the assay is shorter for Xpert Ultra (around 65 to 87 minutes) than for Xpert MTB/RIF (112 minutes) (Global Laboratory Initiative 2017). Currently, the manufacturer, Cepheid Incorporated (Sunnyvale, CA, USA), has made no claim for the use of Xpert Ultra and Xpert MTB/RIF in non-sputum specimens (Cepheid 2019). However, there is a standard operating procedure provided by WHO for processing non-sputum specimens (WHO 2014).

\section{Clinical pathway}

Xpert Ultra and Xpert MTB/RIF are used for the diagnosis of extrapulmonary tuberculosis and rifampicin resistance. Figure 1 shows the clinical pathway and presents the context in which Xpert Ultra or Xpert MTB/RIF might be used (WHO Operational Handbook Diagnosis (Module 3) 2020). The target conditions were extrapulmonary tuberculosis, which includes several forms 
(e.g. tuberculous meningitis, pleural tuberculosis) and rifampicin resistance.

Figure 1. The clinical pathway describes how patients might present and the point in the pathway at which they would be considered for testing with Xpert Ultra or Xpert MTB/RIF. This algorithm for the use of a molecular WHO-recommended rapid diagnostic (WRD), which includes Xpert Ultra and Xpert MTB/ RIF, comes from the WHO operational handbook on tuberculosis (WHO Operational Handbook Diagnosis (Module 3) 2020). Copyright @ [2020] [World Health Organization]: reproduced with permission. Abbreviations: DST: drug susceptibility testing; INH: isoniazid; MDR-TB: multidrug-resistant TB; MTB: Mycobacterium tuberculosis; PLHIV: people living with HIV; RIF: rifampicin; TB: tuberculosis; WRD: WHO-recommended rapid diagnostic, which includes Xpert Ultra and Xpert MTB/ RIF.

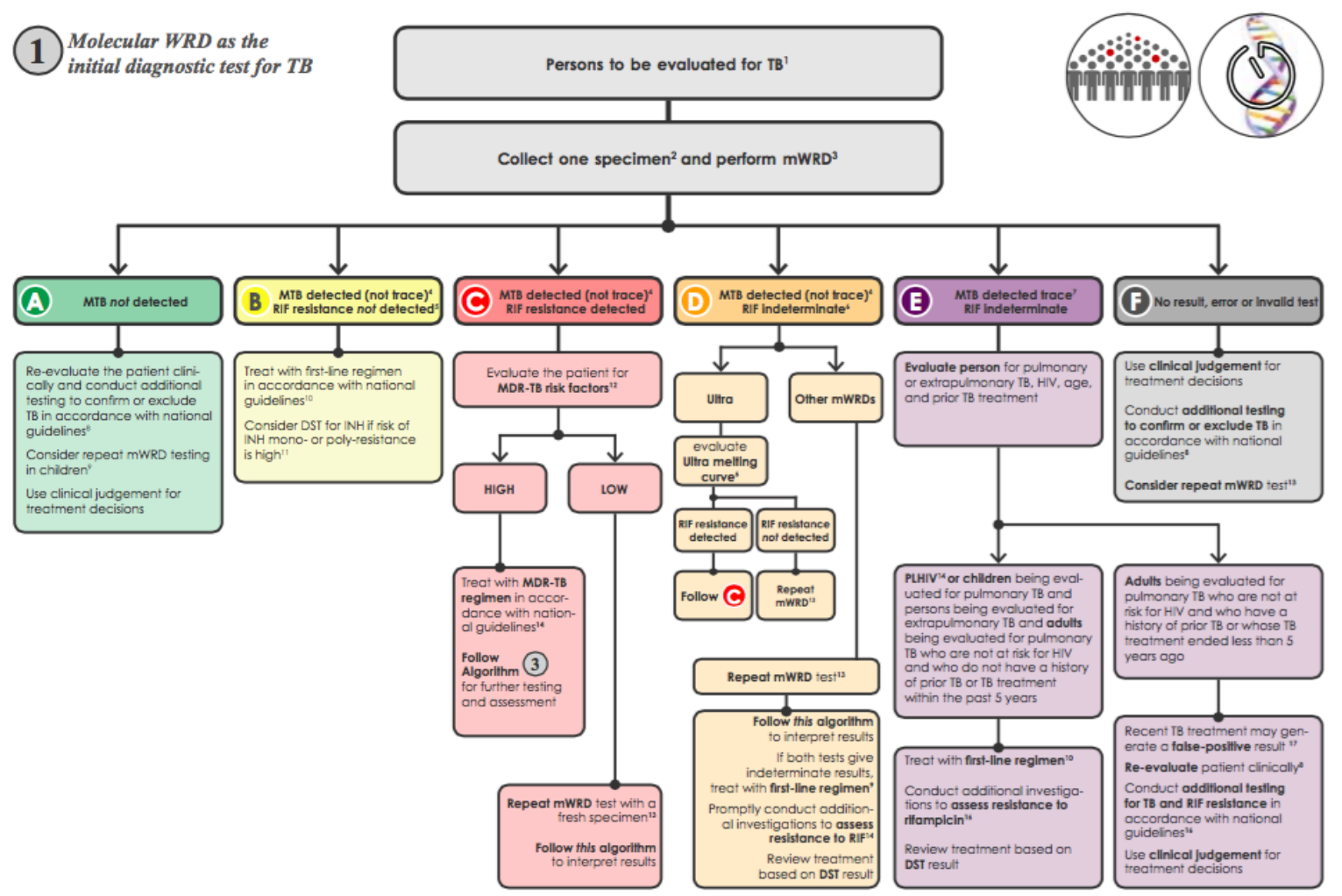

Before a specimen is tested, patients with presumptive extrapulmonary tuberculosis would have undergone a health examination (history and physical examination) and possibly a chest radiograph. The presentation of extrapulmonary tuberculosis varies depending on the body site affected, and it may imitate other diseases, such as cancer and bacterial and fungal infections. Signs and symptoms of extrapulmonary tuberculosis are often non-specific and may include fever, night sweats, fatigue, loss of appetite, and weight loss (as seen in pulmonary tuberculosis) or specific complaints related to the involved site (e.g. headache for tuberculous meningitis, back pain for tuberculosis of the spine). The clinical presentation of extrapulmonary disease may be acute but is more often subacute (falling between acute and chronic) or chronic, meaning that patients may have symptoms for days to months before they seek care.
We have described in Table 1 signs and symptoms of the forms of extrapulmonary tuberculosis included in this review. The clinician should take a careful history, noting history of tuberculosis exposure, prior tuberculosis disease, and medical conditions that increase the risk for tuberculosis disease (e.g. HIV, diabetes mellitus, low body weight). In comparison with HIV-negative people, HIV-positive people have higher rates of extrapulmonary tuberculosis or mycobacteraemia (tuberculosis bloodstream infection). HIV-positive patients with signs or symptoms of extrapulmonary tuberculosis should have specimens taken from the suspected site(s) of involvement to increase the likelihood of tuberculosis diagnosis. Tuberculous meningitis is the most severe form of tuberculosis. In tuberculous meningitis, diagnosis is often delayed, with appalling consequences for patients. For all forms of extrapulmonary tuberculosis, patients may be evaluated in primary- or secondary-care settings. However, 
if more complex or invasive tests are needed, patients may be referred to a tertiary medical centre (Iseman 2000; Reuter 2009; Sharma 2004).

The downstream consequences of testing include the following.

- True-positive (TP): patients would benefit from rapid diagnosis and appropriate treatment.

- True-negative (TN): patients would be spared unnecessary treatment and would benefit from reassurance and pursuit of an alternative diagnosis.

- False-positive (FP): patients would likely experience anxiety and morbidity caused by additional testing, unnecessary treatment, and possible adverse effects; possible stigma associated with a tuberculosis or MDR-TB diagnosis; and the chance that a falsepositive may halt further diagnostic evaluation.

- False-negative (FN): increased risk of morbidity and mortality and delayed treatment initiation for patients.

\section{Role of index test(s)}

We were interested in the following roles for testing.

\section{Xpert Ultra and Xpert MTB/RIF for detection of extrapulmonary tuberculosis}

Index test used as an initial test replacing usual practice (including conventional microscopy, culture or histopathology) for the diagnosis of extrapulmonary tuberculosis in adults with presumptive extrapulmonary tuberculosis (WHO Consolidated Guidelines (Module 3) 2020). An initial test does not mean that other tests will follow.

\section{Xpert Ultra and Xpert MTB/RIF for detection of rifampicin resistance}

Index test used as an initial test replacing culture and phenotypic DST for the diagnosis of rifampicin-resistant tuberculosis in adults with presumptive extrapulmonary tuberculosis (WHO Consolidated Guidelines (Module 3) 2020).

As mentioned, in high MDR-TB settings the presence of rifampicin resistance alone may serve as a proxy for MDR-TB. Xpert Ultra and Xpert MTB/RIF do not eliminate the need for subsequent culture and phenotypic DST, which are required to monitor treatment progress and to detect resistance to drugs other than rifampicin.

\section{Alternative test(s)}

For a comprehensive review of new tests not yet in widespread use, we refer the reader to Branigan 2019; Lewinsohn 2017; Unitaid 2017.

Smear microscopy (light microscopy (Ziehl-Neelsen), fluorescence microscopy, or light-emitting diode (LED) fluorescence microscopy) is the examination of smears for acid-fast bacilli (tuberculosis bacteria) under a microscope. Around 5000 to 10,000 organisms per $\mathrm{mL}$ must be present in the specimen for tuberculosis bacteria to be visible by microscopy (American Thoracic Society 2000). For extrapulmonary tuberculosis, microscopy can be performed in fluid or tissue specimens from sites of disease involvement, for example, in cerebrospinal fluid (CSF) in presumptive tuberculous meningitis or in lymph node tissue in presumptive lymph node tuberculosis. For most extrapulmonary sites, because there are usually few organisms, the sensitivity of smear microscopy is generally low.
Ranges from studies, some with selected cases, are quoted here: $0 \%$ to $10 \%$ in pleural fluid; $14 \%$ to $39 \%$ in pleural tissue; $2 \%$ to $30 \%$ in CSF; $<5 \%$ in peritoneal fluid; and $0 \%$ to $42 \%$ in pericardial fluid. In contrast, the specificity of smear microscopy tends to be quite high, as can be seen in pulmonary tuberculosis $(\geq 90 \%$ ) (Kilpatrick 1986; Lewinsohn 2017).

Mycobacterial culture is a method used to grow bacteria on nutrient-rich media. In comparison with microscopy, a positive culture requires only around 100 organisms per $\mathrm{mL}$ and therefore can detect lower numbers of tuberculosis bacteria (American Thoracic Society 2000). Additionally, culture is essential for species identification and DST. However, culture takes several weeks and requires a highly-equipped laboratory. Culture has reduced sensitivity in paucibacillary disease (reference standards have included culture from a different specimen, such as sputum, smear microscopy, NAATs, presence of granulomatous inflammation, clinical criteria, imaging studies, and response to anti-tuberculosis therapy, done alone or in various combinations): CSF $45 \%$ to $70 \%$; pleural fluid $23 \%$ to $58 \%$; urine $80 \%$ to $90 \%$; peritoneal tuberculosis $45 \%$ to $69 \%$; pericardial tuberculosis $50 \%$ to $65 \%$ (Lewinsohn 2017); lymph node tuberculosis (excisional biopsy) $18 \%$ to $93 \%$; and lymph node tuberculosis (fine-needle aspirate) $10 \%$ to $67 \%$ (Fontanilla 2011).

Histological examination involves examination of tissue specimens under a microscope. Diagnosis of extrapulmonary tuberculosis by histological examination is based on finding acid-fast bacilli and granulomatous inflammation, frequently with caseous (cheese-like) necrosis (necrotizing granulomas). The sensitivity of histology has been reported to vary for different forms of extrapulmonary tuberculosis (reference standards have included smear microscopy, culture, NAATs, clinical criteria, and imaging studies, done alone or in various combinations): $59 \%$ to $88 \%$ for lymph node tuberculosis (excisional biopsy) (Fontanilla 2011); 69\% to $97 \%$ in pleural tissue (closed pleural biopsy); $86 \%$ to $94 \%$ in urological tissue; $60 \%$ to $70 \%$ in endometrial curettage; $79 \%$ to $100 \%$ in peritoneal biopsy; and $73 \%$ to $100 \%$ in pericardial tissue (Lewinsohn 2017). Sensitivity has also been observed to vary for different diagnostic techniques. Diacon 2003 found thoracoscopy to be more sensitive (sensitivity of $100 \%$ ) than closed-needle biopsy (sensitivity of $66 \%$ ) for establishing a diagnosis of pleural tuberculosis (reference standards have included microscopy smear, culture, or presence of granulomatous inflammation with caseous necrosis). Specificity has been observed to be low because of the presence of granulomas in other diseases, both infectious and non-infectious (Lewinsohn 2017), although the presence of 'necrotizing' granulomatous inflammation increases specificity (Woodard 1982). Histological examination carries the additional concern that invasive procedures that are complex and costly may be required to obtain the necessary specimens (Golden 2005).

Cytopathological examination of fluid specimens (such as pleural and peritoneal fluid) may be performed, first to exclude cancer, and then to obtain material for additional analyses, such as measurement of levels of adenosine deaminase and free interferon-gamma (IFN- $\gamma$ ) and cell counts (Lewinsohn 2017; Wright 2009a). Advantages of these tests include that they are rapid and simple and can be performed in most clinical laboratories (Dinnes 2007). In pleural, pericardial, and peritoneal fluid, a predominance of lymphocytes, especially in the absence of mesothelial cells, is highly suggestive of tuberculosis (Wright 2009a). However, in HIV- 
positive people, this pattern may not be observed (Wright 2009a). Adenosine deaminase, an enzyme involved in purine metabolism, has been extensively studied for its potential role in the diagnosis of pleural tuberculosis, peritoneal tuberculosis, and tuberculous meningitis (Lewinsohn 2017). IFN- $\gamma$ is released after it is sensitized by $T$ cells in response to specific $M$ tuberculosis antigens. A recent review of the evidence using GRADE provides the following recommendations.

- $\quad$ "...cell counts and chemistries be performed on amenable fluid specimens (including include pleural, cerebrospinal, ascitic, and joint fluid) collected from sites of suspected extrapulmonary TB (conditional recommendation, very low-quality evidence).

- ...adenosine deaminase levels be measured, rather than not measured, on fluid collected from patients with suspected pleural TB, TB meningitis, peritoneal TB, or pericardial TB (conditional recommendation, low-quality evidence).

- ...free IFN- $\gamma$ levels be measured, rather than not measured, on fluid collected from patients with suspected pleural TB or peritoneal TB (conditional recommendation, low-quality evidence)" (Lewinsohn 2017).

NAAT is a molecular technique that can detect small quantities of genetic material (DNA or RNA) from micro-organisms, such as $M$ tuberculosis. The key advantage of NAATs is that they are rapid diagnostic tests, potentially providing results in a few hours. This is a particularly important feature of the test in life-threatening forms of extrapulmonary tuberculosis, such as tuberculous meningitis. A variety of molecular amplification methods are available, of which PCR is the most common. NAATs are available as commercial kits and in-house tests (based on a protocol developed in a laboratory) and are used routinely in high-income countries for tuberculosis detection. In-house PCR is widely used in low-income countries because these tests are less expensive than commercial kits. An older editorial summarizing three systematic reviews (140 studies) of commercial and in-house NAATs (other than Xpert MTB/RIF) for different forms of extrapulmonary tuberculosis found relatively low sensitivity and underscored concerns about the cost and feasibility of this technology in resource-limited areas (Pai 2008). Similarly, another systematic review found that NAATs have relatively low sensitivity for extrapulmonary tuberculosis but high specificity (e.g. for tuberculous meningitis, for pleural TB), indicating that these tests cannot be used reliably to rule out tuberculosis (Dinnes 2007). A recent evidence synthesis reported sensitivities of $72 \%$ to $88 \%$ in lymph node tissue, $28 \%$ to $81 \%$ in pleural fluid, $90 \%$ in pleural tissue, and $31 \%$ to $56 \%$ in CSF. Specificity ranged from $90 \%$ to $100 \%$ (Lewinsohn 2017).

Alternative molecular methods for DST include the commercial line-probe assays, GenoType MTBDRplus assay (MTBDRplus, Hain LifeScience, Nehren, Germany), and the Nipro NTM+MDRTB detection kit 2 (Nipro, Tokyo, Japan), which detect the presence of mutations associated with drug resistance to isoniazid and rifampicin (Nathavitharana 2017). MTBDRplus is the most widely studied line-probe assay. Advantages of line-probe assays are that they can provide a result for detection of tuberculosis and drug resistance in one to two days. Drawbacks are that lineprobe assays are expensive and need to be used in intermediate and central laboratories (Unitaid 2017). The WHO recommends that for persons with a sputum smear-positive specimen or a cultured tuberculosis isolate, commercial molecular line-probe assays may be used as the initial test instead of phenotypic culture-based DST to detect resistance to rifampicin and isoniazid (conditional recommendation, moderate certainty in the evidence for the test's accuracy) (WHO Consolidated Guidelines (Module 3) 2020). Other molecular assays for detection of tuberculosis and resistance to rifampicin and isoniazid along with instruments are in development (Walzl 2018).

Alere Determine ${ }^{\text {TM }}$ TB LAM Ag (AlereLAM) Alere Inc, (Waltham, USA) is a commercially-available point-of-care test for tuberculosis disease (pulmonary and extrapulmonary tuberculosis). The test detects lipoarabinomannan (LAM), a component of the bacterial cell wall, which is present in the urine of some people with tuberculosis. AlereLAM is performed by placing urine on one end of a test strip, with results appearing as a band on the strip if tuberculosis is present. The test is simple, requires no special equipment, and shows results in 25 minutes. This urine test has potential advantages over sputum-based testing due to ease of sample collection. The accuracy of urinary LAM detection is improved among people living with HIV with advanced immunosuppression (Bjerrum 2019). In two randomized trials, the use of Alere LAM in HIV-positive adult inpatients was shown to reduce mortality (Gupta-Wright 2018; Peter 2016). Based on evidence from the randomized trials and a Cochrane Review (Bjerrum 2019), the WHO currently recommends that AlereLAM should be used to assist in the diagnosis of active tuberculosis in HIV-positive adults, adolescents, and children (WHO Consolidated Guidelines (Module 3) 2020). The key change from the WHO 2015 guidelines is broadening the indication for use of LF-LAM among HIVpositive inpatients with signs and symptoms of active tuberculosis (pulmonary and extrapulmonary); the test is now recommended for all such patients, irrespective of their CD4 count. The full recommendations, which differ for inpatients and outpatients, are described here (WHO Consolidated Guidelines (Module 3) 2020).

Fujifilm SILVAMP TB LAM (FuijiLAM, co-developed by Foundation for Innovative New Diagnostics (FIND), Geneva, Switzerland and Fujifilm, Tokyo, Japan) is a new, urine-based, point-of-care test for tuberculosis diagnosis in people living with HIV. In an individual participant data meta-analysis that included five cohorts of people living with HIV, FujiLAM was found to have superior sensitivity, $70.7 \%$ ( $95 \% \mathrm{Cl} 59.0 \%$ to $80.8 \%$ ), compared to AlereLAM sensitivity of $42.3 \%$ (31.7\% to $51.8 \%$ ), against a microbiological reference standard; FujiLAM had lower specificity, 90.9\% (87.2 to 93.7), compared to AlereLAM specificity of $95.3 \%$ (92.2 to 97.7) (Broger 2020). At the time of writing, additional prospective clinical trials of FuijiLAM are ongoing to generate data for an updated WHO policy review.

\section{Rationale}

Xpert Ultra and Xpert MTB/RIF are rapid tests that may provide benefits for patients (earlier diagnosis and the opportunity to begin earlier, appropriate treatment), especially in high tuberculosisburden countries.

Since 2010, the WHO has recommended the use of Xpert MTB/RIF as the preferred initial diagnostic test for people thought to have MDR-TB or HIV-associated tuberculosis (strong recommendation, moderate-certainty evidence) (WHO Xpert MTB/RIF Policy 2011). In 2013, the WHO expanded the recommendations, stating that Xpert MTB/RIF may be used rather than conventional microscopy and culture as the initial diagnostic test in all adults suspected of having tuberculosis (conditional recommendation acknowledging 
resource implications, high-quality evidence) (WHO Xpert MTB/ RIF Policy Update 2013). The 2013 recommendations extended to the diagnosis of several forms of extrapulmonary tuberculosis, including tuberculous meningitis and lymph nodes and other tissue. In addition, the WHO recommended that following an Xpert MTB/RIF test that demonstrates rifampicin resistance, subsequent DST (e.g. using a line-probe assay to second-line drugs) remains essential to detect resistance to drugs other than rifampicin (WHO Xpert MTB/RIF Policy Update 2013). In 2017, based on a noninferiority analysis of Xpert Ultra compared with Xpert MTB/RIF (Dorman 2018), the WHO stated that recommendations on the use of Xpert MTB/RIF also apply to the use of Xpert Ultra as the initial diagnostic test for all adults and children with signs and symptoms of tuberculosis (WHO Xpert Ultra 2017).

In December 2019, the WHO convened a Guideline Development Group to update the recommendations on the use of molecular assays intended as initial tests for the diagnosis of pulmonary and extrapulmonary tuberculosis and rifampicin resistance. To extend the work of our previous Cochane Review (Kohli 2018), we performed this review update to inform the WHO policy (WHO Consolidated Guidelines (Module 3) 2020).

The Background and Methods sections of this review include some text that overlaps with some of our other reviews for Xpert MTB/RIF Ultra and Xpert MTB/RIF for diagnosing tuberculosis (Horne 2019; Kay 2020; Shapiro 2020; Vonasek 2020).

\section{O B J E C T IVES}

To estimate the diagnostic accuracy of Xpert Ultra and Xpert MTB/ RIF for a) extrapulmonary tuberculosis by site of disease and b) rifampicin resistance, in adults with presumptive extrapulmonary tuberculosis. Presumptive tuberculosis refers to a patient who presents with symptoms or signs suggestive of tuberculosis.

\section{Secondary objectives}

- To compare the diagnostic accuracy of Xpert Ultra and Xpert MTB/RIF for a) extrapulmonary tuberculosis by site of disease, and b) rifampicin resistance.

- To investigate the effects of potential sources of heterogeneity on test accuracy across the included studies.

For potential sources of heterogeneity, for extrapulmonary tuberculosis, we included smear status, HIV status, and prevalence of extrapulmonary tuberculosis. For cerebrospinal fluid (CSF), we considered the presence of a concentration step and specimen volume.

For rifampicin resistance, we planned to assess the impact of the prevalence of rifampicin resistance on accuracy estimates, but we had insufficient data for this analysis.

\section{METHODS}

\section{Criteria for considering studies for this review Types of studies}

We included cross-sectional and cohort studies. In addition, we had planned to include randomized controlled trials that evaluated the use of the index(s) test on patient health outcomes, but that also reported sensitivity and specificity. Although the study design was a randomized trial for the purpose of determining the impact of the test on participant outcomes, the study design was a crosssectional study for the purpose of determining the diagnostic accuracy of the index tests in this review. However, we did not identify any randomized controlled trials. We used abstracts to identify published studies and included these when they met the inclusion criteria. We only included studies that reported data comparing the index test(s) to an acceptable reference standard from which we could extract true-positive (TP), true-negative (TN), false-positive (FP), and false-negative (FN) values. We excluded case-control studies and case reports.

\section{Participants}

We included studies where at least $85 \%$ of the participants enrolled were adults aged 15 years or older with presumptive extrapulmonary tuberculosis from all settings and countries. Restricting the age group to adults differs from the original review, where we also included children (Kohli 2018). We did this because children are now included in a separate Cochrane Review (Kay 2020). We excluded studies where we could not disaggregate data on adults from those in children and studies where we could not tell the age of the participants enrolled.

We included non-respiratory specimens (such as CSF, pleural fluid, lymph node aspirate or tissue). We excluded sputum and other respiratory specimens, such as fluid obtained from bronchial alveolar lavage and tracheal aspiration. As we anticipated finding many studies, we set a bar to exclude smaller studies to reduce unnecessary work. We therefore required studies to provide data for at least five specimens for a given form of extrapulmonary tuberculosis included in the review. We excluded studies evaluating the use of Xpert Ultra and Xpert MTB/RIF to diagnose relapse of previously-treated extrapulmonary tuberculosis, so as to avoid the selection bias that may arise by limiting to a group that is already at elevated risk of extrapulmonary tuberculosis. We attempted to identify studies that included participants who were not taking anti-tuberculosis drugs or had taken anti-tuberculosis drugs for less than seven days.

\section{Index tests}

The index tests were Xpert Ultra and Xpert MTB/RIF.

Index test results are automatically generated (i.e. there is a single threshold), and the user is provided with a printable test result as follows.

\section{Xpert Ultra}

- MTB (M tuberculosis) DETECTED HIGH; RIF (rifampicin) Resistance DETECTED

- MTB DETECTED MEDIUM; RIF Resistance DETECTED

- MTB DETECTED LOW; RIF Resistance DETECTED

- MTB DETECTED VERY LOW; RIF Resistance DETECTED

- MTB DETECTED HIGH; RIF Resistance NOT DETECTED

- MTB DETECTED MEDIUM; RIF Resistance NOT DETECTED

- MTB DETECTED LOW; RIF Resistance NOT DETECTED

- MTB DETECTED VERY LOW; RIF Resistance NOT DETECTED

- MTB DETECTED HIGH; RIF Resistance INDETERMINATE

- MTB DETECTED MEDIUM; RIF Resistance INDETERMINATE

- MTB DETECTED LOW; RIF Resistance INDETERMINATE 
- MTB DETECTED VERY LOW; RIF Resistance INDETERMINATE

- MTB Trace DETECTED; RIF Resistance INDETERMINATE

- INVALID (the presence or absence of MTB cannot be determined)

- ERROR (the presence or absence of MTB cannot be determined)

- NO RESULT (the presence or absence of MTB cannot be determined)

Xpert Ultra incorporates a semi-quantitative classification for results: trace, very low, low, moderate, and high. 'Trace' corresponds to the lowest bacterial burden for detection of $M$ tuberculosis (Chakravorty 2017). We considered a trace result to mean MTB ( $M$ tuberculosis) DETECTED. However, no rifampicinresistance result was available for participants with trace results because the trace sample is always reported as 'INDETERMINATE' for rifampin resistance (Cepheid 2018).

\section{Xpert MTB/RIF}

- MTB (M tuberculosis) DETECTED; Rif (rifampicin) resistance DETECTED

- MTB DETECTED; Rif resistance NOT DETECTED

- MTB detected; Rif resistance INDETERMINATE

- MTB NOT DETECTED

- INVALID (the presence or absence of MTB cannot be determined)

- ERROR (the presence or absence of MTB cannot be determined)

- NO RESULT (the presence or absence of MTB cannot be determined)

\section{Target conditions}

The target conditions were extrapulmonary tuberculosis and rifampicin resistance. We included eight common forms of extrapulmonary tuberculosis and considered subcategories of the target condition as separate diagnostic classifications (CDC 2018; Sandgren 2013; Sharma 2004).

- Tuberculous meningitis.

- Pleural tuberculosis.

- Lymph node tuberculosis.

- Genitourinary tuberculosis.

- Bone or joint tuberculosis.

- Peritoneal tuberculosis.

- Pericardial tuberculosis.

- Disseminated tuberculosis.

Table 1 lists the forms of extrapulmonary tuberculosis and specimens used for diagnosis in the review. We excluded less common forms, such as cutaneous tuberculosis, ocular tuberculosis, female genital tuberculosis, and tuberculosis of the breast, ear, and paranasal sinuses (Sharma 2004).

\section{Reference standards}

\section{Detection of extrapulmonary tuberculosis}

We included two reference standards.
- Solid or liquid mycobacterial culture.

* 'Tuberculosis' was defined as a positive $M$ tuberculosis culture

* 'Not tuberculosis' was defined as a negative $M$ tuberculosis culture

- Composite reference standard.

* 'Tuberculosis' was defined as a positive $M$ tuberculosis culture or positive composite reference test.

* 'Not tuberculosis' was defined as a negative $M$ tuberculosis culture and a negative composite reference test.

The composite reference standard might be based on the results of microbiological tests, culture or NAAT other than Xpert Ultra and Xpert MTB/RIF; imaging studies; histology; and clinical characteristics, and include at least one component test that is positive, according to the definition of the primary study authors.

For pleural tuberculosis, we defined the composite reference standard as the presence of granulomatous inflammation or a positive culture. We proposed this definition because we found evidence to support including histopathological examination in the definition. Around $60 \%$ of patients undergoing pleural biopsy will show granulomatous inflammation (American Thoracic Society 2000). In a prospective cohort study of participants with clinical and radiological findings consistent with pleural tuberculosis, Conde 2003 found that histological examination of tissue obtained from pleural biopsy had a higher diagnostic yield $(78 \% ; 66 / 84)$ than that of culture $(62 \% ; 52 / 84)$.

Culture is considered the best reference standard for tuberculosis. However, culture may lead to misclassification of some cases of extrapulmonary tuberculosis as 'not tuberculosis', owing to the paucibacillary nature of the disease. This means that culture may have low sensitivity for extrapulmonary tuberculosis overall and further that culture sensitivity may differ for different forms of extrapulmonary tuberculosis (Lewinsohn 2017). This misclassification by culture may lead to biased estimates (overestimation or underestimation) of the diagnostic accuracy of Xpert Ultra and Xpert MTB/RIF. The extent of bias will depend on the frequency of errors by culture and the degree of correlation in errors by culture and the Xpert assays because culture and Xpert Ultra or Xpert MTB/RIF are likely to pick up cases with a higher bacterial load, and are likely to miss cases with a lower bacterial load. Ignoring this dependence could lead to an overestimation of the sensitivity of Xpert Ultra or Xpert MTB/RIF.

- Effect of low sensitivity of culture on Xpert sensitivity: the low sensitivity of culture means that index test FNs may be misclassified as TNs when culture is used as the reference standard. Therefore, when Xpert Ultra or Xpert MTB/RIF is evaluated against culture, the number of FNs (classified as negative by the index test and positive by the reference test) may be decreased and the sensitivity of the index test may be overestimated.

- Effect of low sensitivity of culture on Xpert specificity: the low sensitivity of culture means that index test TPs may be misclassified as FPs when culture is used as the reference standard. Therefore, when Xpert Ultra or Xpert MTB/RIF is evaluated against culture, the number of FPs (classified as positive by the index test and negative by the reference test) may be increased and specificity of the index test may be underestimated. 
In contrast to culture, a composite reference standard that includes culture, other tests, and clinical characteristics may correctly classify index test results as TPs (instead of as FPs with respect to culture), especially in people with paucibacillary disease in whom culture may be negative. However, because of the uncertainties that surround a clinical diagnosis of tuberculosis and, in some instances, the conditional dependence of the index tests and other tests in the composite reference standard (for example, for most of these tests, detection of tuberculosis depends on bacillary load), a reference standard that uses additional tests and clinical characteristics (in culture-negative people) may incorrectly classify people without tuberculosis as having tuberculosis (Naaktgeboren 2013). An additional challenge with including a composite reference standard is that the definition of the composite reference standard may vary across studies, making it difficult to interpret the accuracy estimates.

Thus both reference standards, culture and composite, are imperfect and may affect accuracy estimates. In an attempt to improve the estimation of diagnostic accuracy, we applied a latent class meta-analysis model to the three most commonly studied forms of extrapulmonary tuberculosis. This approach provides the sensitivity and specificity of culture in addition to the accuracy of the index tests, thus adjusting for imperfect culture accuracy.

\section{Detection of rifampicin resistance}

The reference standard was culture-based DST using solid or liquid media or line-probe assays, as recommended by the WHO (WHO 2012; WHO Consolidated Guidelines (Module 3) 2020).

\section{Search methods for identification of studies}

We attempted to identify all relevant studies, regardless of language or publication status (published, unpublished, in press, or ongoing). We monitored abstracts to see if these studies were published during the time we performed the review. We included only published studies in the review.

\section{Electronic searches}

For the original review, we searched the literature on 7 August 2017. For this review update, we searched the literature on 2 August 2019 and again on 28 January 2020, specifically for studies of Xpert Ultra (studies could include Xpert Ultra alone or both Xpert Ultra and Xpert MTB/RIF), using the search terms and strategy described in Appendix 1. We searched the following databases:

- Cochrane Infectious Diseases Group Specialized Register;

- MEDLINE (OVID, from 1966);

- Embase (OVID, from 1974);

- Science Citation Index - Expanded (from 1900);

- Conference Proceedings Citation Index - Science (CPCI-S, from 1990);

- BIOSIS Previews (from 1926), all three from the Web of Science;

- Scopus (Elsevier, from 1970);

- Latin American Caribbean Health Sciences Literature (LILACS) (BIREME, from 1982).

We also searched ClinicalTrials.gov, the WHO International Clinical Trials Registry (ICTRP) Platform (www.who.int/trialsearch), and the International Standard Randomized Controlled Trials Number (ISRCTN) registry (www.isrctn.com/) for trials in progress, and ProQuest Dissertations \& Theses A\&l (www.proquest.com/ pqdtglobal, from 1990) for dissertations.

To identify other systematic reviews and meta-analyses, we performed an additional search on 28 May 2020 in MEDLINE (PubMed), Embase (OVID), and the Cochrane Library, applying filters for systematic reviews (www.sign.ac.uk/search-filters.html) to search terms for Xpert and tuberculosis.

\section{Searching other resources}

We reviewed reference lists of included articles and any relevant review articles identified through the above methods. We also contacted researchers at FIND and other experts in the field of tuberculosis diagnostics for information on ongoing and unpublished studies.

\section{Data collection and analysis}

\section{Selection of studies}

We used Covidence to manage the selection of studies (Covidence 2017). Two review authors independently scrutinized titles and abstracts identified by electronic literature searching to identify potentially eligible studies. We selected any citation identified by either review author as potentially eligible for full-text review. The same review authors independently assessed full-text papers for study eligibility using predefined inclusion and exclusion criteria, and resolved any discrepancies by discussion. We recorded all studies excluded after full-text assessment and their reasons for exclusion in Characteristics of excluded studies. We illustrated the study selection process in a PRISMA diagram (Moher 2009).

\section{Data extraction and management}

Using a previously-developed form (Appendix 2), two review authors worked independently to extract data on the following characteristics.

- Author; publication year; country; setting (outpatient, inpatient, or both outpatient and inpatient); study design; manner of participant selection; number of participants enrolled; number of participants for whom results are available.

- Characteristics of participants: gender; age; HIV status; history of prior tuberculosis; receipt of anti-tuberculosis treatment.

- Index test.

- Target condition and subcategories.

- Type of reference standard.

- Quality Assessment of Studies of Diagnostic Accuracy - Revised (QUADAS-2) items.

- Details of specimen: type (such as CSF, pleural fluid, or lymph node aspirate or tissue); condition (fresh or frozen); smearpositive or smear-negative.

- Specimen preparation; homogenization step (for tissue specimens); concentration step and specimen volume (for CSF); adherence to WHO standard operating procedures.

- Number of TP, FP, FN, TN (i.e. true-positives, false-positives, false-negatives, and true-negatives), and trace results; number of inconclusive results for detection of extrapulmonary tuberculosis; number of indeterminate results for detection of rifampicin resistance.

- Number of missing or unavailable test results. 
We classified country income status as either low- and middleincome or high-income, according to the World Bank List of Economies (World Bank 2020).

We extracted TP, FP, FN, and TN values for the following specimens: CSF, pleural fluid and tissue, lymph node aspirate and tissue (the latter specimen acquired by surgical biopsy), bone or joint aspirate and tissue, urine, peritoneal fluid and tissue, pericardial fluid and tissue, and blood. We extracted these values for each of the specimen types separately. For example, we used one $2 \times 2$ table for lymph node aspirate, and another $2 \times 2$ table for lymph node tissue. In situations in which a participant contributed more than one specimen but of different types, we extracted data for all specimens. When a study included data for both raw specimens and concentrated sediment involving the same participants, we preferentially extracted data for raw specimens, except in the case of CSF, for which we extracted data for concentrated sediment as recommended by the WHO (WHO 2014). We extracted accuracy data according to the defined reference standards (see Reference standards). We did not encounter any situations in which a subset of participants in a study received the reference standard but others did not. Hence, there was no need to make corrections for verification bias in the statistical analysis (Begg 1983).

In most studies, the number of specimens was the same as the number of participants. However, in some studies, the number of specimens exceeded the number of participants or study authors reported only the number of specimens. In the previous review (Kohli 2018), we added post hoc a sensitivity analysis limiting inclusion to studies that used one specimen per participant. In this review, we performed a similar sensitivity analysis for Xpert Ultra.

We contacted authors of primary studies for missing data or clarifications. We entered all data into Microsoft Excel 2014.

\section{Assessment of methodological quality}

We used the QUADAS-2 tool, tailored to this review, to assess the quality of the included studies (Appendix 3) (Whiting 2011). QUADAS-2 consists of four domains: patient selection, index test, reference standard, and flow and timing. We assessed all domains for risk of bias and the first three domains for concerns about applicability. Two review authors independently completed QUADAS-2 and resolved disagreements through discussion. We present the results of this quality assessment in text, tables, and graphs.

We followed Cochrane policy, which states that "authors of primary studies will not extract data from their own study or studies. Instead, another author will extract these data, and check the interpretation against the study report and any available study registration details or protocol".

\section{Statistical analysis and data synthesis}

We performed descriptive analyses of the characteristics of included studies using Stata 15 (Stata 2017), and we present key study characteristics in the Characteristics of included studies table. We used data reported in the TP, FP, FN, and TN format to calculate sensitivity and specificity estimates and $95 \%$ confidence intervals (Cls) for individual studies. We present individual study results graphically by plotting the estimates of sensitivity and specificity (and their $95 \% \mathrm{Cls}$ ) in forest plots and receiver operating characteristic (ROC) space using Review Manager 5 (RevMan 5) (RevMan 2014).

When data were sufficient, we performed meta-analyses to estimate pooled sensitivity and specificity and corresponding 95\% credible interval ( $\mathrm{Crl}$, defined below) using an adaptation of the bivariate random-effects approach of Reitsma 2005, which uses the exact binomial likelihood for the observed proportions (Chu 2006). The bivariate random-effects approach allowed us to calculate the pooled estimates of sensitivity and specificity while dealing with potential sources of variation caused by (1) imprecision of sensitivity and specificity estimates within individual studies; (2) correlation between sensitivity and specificity across studies; and (3) variation in sensitivity and specificity between studies. The model has a hierarchical structure, with the logit sensitivity in individual studies assumed to come from a common probability distribution the mean of which is the pooled logit sensitivity, and the standard deviation is the between-study standard deviation, and likewise for the specificity. This structure allows for borrowing strength across studies. In the absence of sufficient studies, we simply present descriptive statistics. In addition, we determined predictive values at a pretest probability of $10 \%$, a value suggested by the WHO.

We performed separate analyses grouped by type of extrapulmonary specimen (e.g. CSF, pleural fluid, peritoneal fluid) rather than determine summary accuracy estimates for all forms of extrapulmonary tuberculosis combined, because we considered the former approach to be most clinically meaningful. In addition, we performed separate analyses by reference standard.

\section{Comparison of Xpert Ultra and Xpert MTB/RIF}

We performed comparative meta-analyses by restricting the analyses to only those studies that made direct comparisons between Xpert Ultra and Xpert MTB/RIF within the same participants (Takwoingi 2013). We extracted the median and the $95 \% \mathrm{Crl}$ for the difference in the pooled sensitivities and the difference in the pooled specificities, respectively, of Xpert Ultra versus Xpert MTB/RIF. We also calculated the probability that the difference exceeds zero in each case.

For analysis of Xpert MTB/RIF or Xpert Ultra accuracy for detection of rifampicin resistance, we include participants who (1) were culture-positive; (2) had a valid culture-based DST or lineprobe assay (LPA) result; (3) were Xpert MTB/RIF or Xpert Ultra tuberculosis-positive; and (4) had a valid Xpert MTB/RIF or Xpert Ultra result for rifampicin resistance, detected or not detected (susceptible).

- Sensitivity $=$ Xpert MTB/RIF (or Xpert Ultra) rifampicin resistance detected/phenotypic DST or LPA rifampicin-resistant.

- Specificity $=$ Xpert MTB/RIF (or Xpert Ultra) rifampicin resistance not detected/phenotypic DST or LPA rifampicin-susceptible.

For detection of rifampicin resistance, when a study included multiple types of specimens, we based our determination of Xpert Ultra and Xpert MTB/RIF and sensitivity and specificity on all available data in the study, including data for specimens that we did not include in the primary analyses for detection of extrapulmonary tuberculosis. For example, if a study provided data for several specimen types combined (e.g. all tissue specimens) and we could not disaggregate the data for a specific specimen type, we included 
all data (for all tissue specimens) in the analysis for rifampicin resistance detection. We did this because we did not expect the accuracy of Xpert Ultra or Xpert MTB/RIF for rifampicin resistance to vary by specimen type. We used the bivariate random-effects model to estimate pooled sensitivity and specificity.

We estimated all models using a Bayesian approach with lowinformation prior distributions using OpenBUGS software (Version 3.2.3) (Lunn 2009), along with $\mathrm{R}$ (R Core Team 2019). Under the Bayesian approach, all unknown parameters must be provided a prior distribution that defines the range of possible values of the parameter and the weight of each of those values, based on information external to the data. To allow observed data to dominate the final results, we chose to use low-information prior distributions. We defined prior distributions on the logodds scale over the pooled sensitivity and specificity parameters, their corresponding between-study standard deviations, and the correlation between the sensitivities and specificities across studies. For the pooled log odds of the sensitivity or the pooled log odds of the specificity, we used a normal prior distribution with mean 0 and a wide variance of 4 (or a precision of 0.25 ). This corresponds to a roughly uniform distribution over the pooled sensitivity and pooled specificity on the probability scale. For the between-study precision, we used a gamma distribution with a shape parameter of 2 and a rate parameter of 0.5 . This corresponds to a $95 \%$ prior $\mathrm{Crl}$ for the between-study standard deviation in the log odds of sensitivity or the log odds of specificity ranging from roughly 0.29 to 1.44 , corresponding to moderate to high values of between-study heterogeneity. Covariance terms followed a uniform prior distribution whose upper and lower limits were determined by the sensitivity of the two tests. The OpenBUGS model used appears in Appendix 4. It is known that meta-analysis models can be sensitive to the choice of prior distributions over between-study standard deviation parameters. We therefore carried out sensitivity analyses and considered alternative prior distributions that are less informative, allowing a wider range of possible values. To study the sensitivity of all results to the choice of prior distributions given above, we considered alternative prior distributions that were less informative, allowing a wider range of possible values. We increased the variance of the normal distributions over the pooled log odds of sensitivity or specificity to 100 . We used a uniform prior distribution ranging from 0 to 3 over the between-study standard deviation on the log odds scale (see programme in Appendix 4). We noted no appreciable change in pooled accuracy parameters but found that the posterior Crls and prediction intervals were slightly wider, as expected.

We combined information from the prior distribution with the likelihood of the observed data, in accordance with Bayes' theorem, using the OpenBUGS programme, which provides a sample from the posterior distribution of each unknown parameter. We were particularly interested in the pooled sensitivity and specificity of Xpert and between-study variance in the sensitivity and specificity of Xpert on the log-odds scale. Using a sample from the posterior distribution, we calculated various descriptive statistics of interest. We estimated the median pooled sensitivity and specificity and their $95 \% \mathrm{Crl}$. The median or the $50 \%$ quantile is the value below which $50 \%$ of the posterior sample lies. We report the median because the posterior distributions of some parameters may be skewed and the median would be considered a better point estimate of the unknown parameter than the mean in such cases. The $95 \% \mathrm{Crl}$ is the Bayesian equivalent of the classical (frequentist) 95\% confidence interval $(\mathrm{Cl})$ (we will indicate 95\% $\mathrm{Cl}$ for individual study estimates and $95 \% \mathrm{Crl}$ for pooled study estimates as appropriate). The $95 \% \mathrm{Crl}$ may be interpreted as an interval that has a $95 \%$ probability of capturing the true value of the unknown parameter, given observed data and prior information. We prepared summary receiver operating characteristic (SROC) curves for each meta-analysis model, using the methods described in Harbord 2007.

We also determined the predicted sensitivity and specificity of Xpert MTB RIF and Xpert Ultra and their 95\% Crls. Predicted values represent our best guess for sensitivity and specificity in a future study and will be close to the pooled estimates. However, their Crls may be different. If there is no heterogeneity at all between studies, the $\mathrm{Crl}$ around the predicted estimate will be the same as the $\mathrm{Crl}$ around the pooled estimate. On the other hand, if considerable heterogeneity is observed between studies, the $\mathrm{Crl}$ around the predicted estimate will be much wider than the $\mathrm{Cl}$ around the pooled estimate.

In addition, we performed latent class analysis for three forms of extrapulmonary tuberculosis: tuberculous meningitis, pleural tuberculosis, and lymph node tuberculosis, using data from the two-by-two tables comparing the index test to culture as a reference standard. Latent class analysis is a statistical modelling technique that allows estimation of test accuracy in the absence of an adequate reference standard to define the presence or absence of disease (Van Smeden 2014). The latent class metaanalysis model expands the traditional meta-analysis model in two ways: (1) we added parameters for the sensitivity and specificity of culture; and (2) we added covariance terms to adjust for the dependence between the index test and culture among diseasepositive and disease-negative participants in each study. We used hierarchical prior distributions over the logit sensitivity and logit specificity of culture. In other words, we assumed that the logit sensitivities in the individual studies come from a common probability distribution whose mean is the pooled mean logit sensitivity of culture and whose standard deviation is the betweenstudy standard deviation. Likewise for the specificities. We used the same low-information prior distributions over the pooled logit mean and between-study standard deviation parameters as we had for the corresponding parameters for the index test. We used uniform prior distributions for covariance terms over their ranges, which are determined by the sensitivities and the specificities of the two tests in each study (see Appendix 4 for the OpenBUGS model). We found that we did not need to augment observed data with prior information from other sources for most models. However, in a post hoc analysis Xpert MTB/RIF in lymph node aspirate in which we suspected a systematic bias in the performance of culture, we used informative prior distributions over the specificity of culture (ranging from $99 \%$ to $100 \%$ ) and the specificity of Xpert MTB/RIF (ranging from $98 \%$ to $100 \%$ ) (see Appendix 4). We added the SROC plots of the latent class meta-analyses to the SROC plots resulting from the models in which culture was treated as a perfect test, so they could be compared.

Based on work evaluating Xpert MTB/RIF for childhood tuberculosis (Schumacher 2016), we anticipated that latent class meta-analyses would lead to a decrease in the estimated pooled sensitivity of Xpert Ultra and Xpert MTB/RIF and an increase in the estimated pooled specificity of Xpert Ultra and Xpert MTB/RIF compared with the primary analyses. In other words, this method should help to 
correct the biases in Xpert Ultra and Xpert MTB/RIF sensitivity and specificity resulting from treating culture as a perfect reference standard, which we detailed earlier in the section on the reference standard.

\section{Approach to inconclusive index test results}

The proportion of inconclusive (non-determinate) rate for detection of pulmonary tuberculosis is the number of tests classified as 'invalid', 'error', or 'no result' divided by the total number of index tests performed. The proportion of inconclusive (indeterminate) rate for detection of rifampicin resistance is the number of tests classified as 'MTB DETECTED; Rif (rifampicin) resistance INDETERMINATE' divided by the total number of index test-positive results. For Xpert Ultra, we determined the proportion of inconclusive index test results $=$ number of inconclusive test results divided by the total number of tests. In our previous review, we used a Bayesian hierarchical model for a single proportion to estimate the pooled proportion of inconclusive MTB/RIF test results (Kohli 2018). We reported these findings again in this review update. As we found very few inconclusive results reported, we excluded these results from the quantitative analysis.

\section{Investigations of heterogeneity}

Initially, we investigated heterogeneity through visual examination of forest plots of sensitivities and specificities and through visual examination of the ROC space of the raw data. When data allowed, we evaluated potential sources of heterogeneity using subgroup analyses and bivariate meta-regression. We included the following covariates.

- HIV status.

- For tuberculous meningitis, concentration step used for preparing specimen (yes or no).

- CSF specimen volume used for Xpert MTB/RIF or Xpert Ultra testing.

We had planned to investigate smear status, history of tuberculosis, and whether WHO standard procedures for preparing tissue specimens were followed. However, we had insufficient data to do this.

The impact of the prevalence of extrapulmonary tuberculosis on sensitivity and specificity is an important consideration. In a post hoc meta-regression analysis, for Xpert MTB/RIF we explored this question for CSF, pleural fluid, and lymph node aspirate. For Xpert Ultra we explored this question for CSF. We did not conduct other analyses, owing to an insufficient number of studies. For detection of rifampicin resistance, owing to a small number of studies, we could not assess the impact of prevalence of rifampicin resistance on accuracy estimates.

\section{Nontuberculous mycobacteria}

Nontuberculous mycobacteria (NTM), such as M avium complex and $M$ intracellulare, constitute a multi-species group of human pathogens that are ubiquitous in water and soil. NTM can cause severe diseases that share clinical signs with tuberculosis but are treated differently. People living with HIV with severe immunosuppression are particularly vulnerable to infections caused by NTM (Gopinath 2010). Previous studies have shown that Xpert does not cross-react with other mycobacterial species (Blakemore 2010; Helb 2010). In our original review, we summarized data for NTM separately by determining the percentage of false-positive Xpert MTB/RIF results in specimens that grew NTMs (Kohli 2018). In this updated review, we therefore summarize data for NTM only for Xpert Ultra.

\section{Sensitivity analyses}

For Xpert Ultra testing in CSF, we performed sensitivity analyses to explore whether the overall findings were robust to potentially influential decisions. We did this by limiting inclusion in the metaanalysis to the following.

- Studies that used consecutive or random selection of participants.

- Studies in which the reference standard results were interpreted without knowledge of the index test results.

- Studies that included only one specimen per participant.

For Xpert Ultra, in CSF, we also planned to perform a sensitivity analysis by limiting studies to those that included only untreated participants. However, we were unable to confirm that studies met this criterion. We planned similar sensitivity analyses for pleural fluid and lymph node aspirate, but these analyses were not carried out owing to an insufficient number of studies. For all other specimen types, we had an insufficient number of studies for sensitivity analyses.

For Xpert MTB/RIF, in the original review we performed sensitivity analyses by type of extrapulmonary specimen and found that for most analyses, the sensitivity analyses made little difference to any of these findings (Kohli 2018). However, for Xpert MTB/RIF in CSF, in comparison with all studies, (sensitivity of $71.1 \%$ (60.9 to 80.4 ), and specificity of $98.0 \%$ (97.0 to 98.8$)$ ), studies that evaluated only one specimen per participant had lower pooled sensitivity at $63.5 \%$ (47.6 to 76.3 ) and lower pooled specificity at $96.1 \%$ (94.2 to 97.4 ).

\section{Assessment of reporting bias}

We did not perform a formal assessment of publication bias using methods such as funnel plots or regression tests because such techniques have not been helpful for diagnostic test accuracy studies (Macaskill 2010).

\section{Summary of findings and assessment of the certainty of the evidence}

We assessed the certainty of evidence using the GRADE approach for diagnostic studies (Balshem 2011; GRADEpro GDT 2015; Schünemann 2008; Schünemann 2016). As recommended, we rated the certainty of evidence as either high (not downgraded), moderate (downgraded by one level), low (downgraded by two levels), or very low (downgraded by more than two levels) based on five domains: risk of bias, indirectness, inconsistency, imprecision, and publication bias. For each outcome, the certainty of evidence started as high when there high-quality studies (cross-sectional or cohort studies) that enrolled participants with diagnostic uncertainty. If we found a reason for downgrading, we used our judgement to classify the reason as either serious (downgraded by one level) or very serious (downgraded by two levels).Two review authors discussed judgments and applied GRADE in the following way (Schünemann 2020a; Schünemann 2020b).

- Assessment of risk of bias. We used QUADAS-2 to assess risk of bias. 
- Indirectness. We assessed indirectness in relation to the population (including disease spectrum), setting, interventions, and outcomes (accuracy measures). We also used prevalence as a guide to whether there was indirectness in the population.

- Inconsistency. GRADE recommends downgrading for unexplained inconsistency in sensitivity and specificity estimates. We carried out prespecified analyses to investigate potential sources of heterogeneity and downgraded when we could not explain inconsistency in the accuracy estimates.

- Imprecision. We considered a precise estimate to be one that would allow a clinically meaningful decision. We considered the width of the $\mathrm{Crl}$ (or $\mathrm{Cl}$ ) and asked, "Would we make a different decision if the lower or upper boundary of the $\mathrm{Cr}$ (or $\mathrm{Cl}$ ) represented the truth?" In addition, we worked out projected ranges for TP, FN, TN, and FP for a given prevalence of tuberculosis and made judgements on imprecision from these calculations.

- Publication bias. We rated publication bias as undetected (not serious) for several reasons: the comprehensiveness of the literature search and extensive outreach to tuberculosis researchers to identify studies; the presence only of studies that produced precise estimates of high accuracy despite small sample size; and our knowledge about studies that were conducted but not published.

For the 'Summary of findings' tables for CSF and pleural fluid, we provide evidence using culture as the reference standard, which is considered the best reference standard for tuberculosis (Lewinsohn 2017). For lymph node aspirate, we provide evidence using a composite reference because, based on findings from the original review (Kohli 2018), we believe a composite reference standard is preferable for estimating accuracy.

\section{RES ULT S}

\section{Results of the search}

We identified and screened a total of 735 records for inclusion in this review update. Of these, we assessed 142 full-text papers against our inclusion criteria. We excluded 120 papers, mainly for the following reasons: study did not evaluate Xpert Ultra $(n=54)$; could not extract $2 \times 2$ values $(n=14)$; inappropriate reference standard $(n=13)$; could not extract data by specimen type $(n=12)$; did not include extrapulmonary specimen $(n=10)$ : duplicate data $(n=4)$; case-control study $(n=4)$; index test other than Xpert MTB/ RIF or Xpert Ultra ( $n=3)$; study included children $(n=2)$; screening study $(n=2)$; case report $(n=1)$; and fewer than five specimens for a given specimen type $(n=1)$. From our previous review, we included 47 studies.

Thus, we included 69 unique studies that met the inclusion criteria in this review update.

Sixty-seven studies evaluated Xpert MTB/RIF (Ablanedo-Terrazas 2014; Ajbani 2018; Al-Ateah 2012; Azevedo 2018; Bahr 2015; Bahr 2017; Bera 2015; Biadglegne 2014; Blaich 2014; Causse 2011; Che 2017; Chen 2019; Chin 2019; Christopher 2013; Cresswell 2018; Cresswell 2020; Dhasmana 2014; Dhooria 2016; Donovan 2020; Du 2015; El-Din 2019; Feasey 2013; Friedrich 2011; Ghariani 2015; Gu 2015; Hanif 2011; Heemskerk 2018; Hillemann 2011; Iram 2015; Kim 2015a; Li 2017; Liang 2019; Ligthelm 2011; Lusiba 2014; Malbruny 2011; Meldau 2014; Meldau 2019; Metcalf 2018; Nataraj 2016; Nhu 2014; Ozkutuk 2014; Pandie 2014; Patel 2013; Peñata 2016; Rakotoarivelo 2018; Rufai 2015; Rufai 2017a; Rufai 2017b; Safianowska 2012; Sarfaraz 2018; Scott 2014; Sharma 2014; Sharma 2016; Sharma 2018; Siddiqi 2019; Sun 2019; Suzana 2016; Tadesse 2015; Trajman 2014; Ullah 2017; Vadwai 2011; Van Rie 2013; Wang 2019; Wang 2020; Wu 2019; Zeka 2011; Zmak 2013). Eleven studies evaluated Xpert Ultra. Of these 11 studies, nine evaluated both Xpert MTB/RIF and Xpert Ultra (Bahr 2017; Chin 2019; Cresswell 2020; Donovan 2020; Meldau 2019; Sun 2019; Wang 2019; Wang 2020; Wu 2019) and two studies evaluated Xpert Ultra alone (Antel 2020; Perez-Risco 2018). All studies but two (one in Spanish: Peñata 2016, and one in Turkish: Ozkutuk 2014), were written in English. Figure 2 shows the flow of studies in the review. We recorded the excluded studies, including those listed in the previous Cochrane Review (Kohli 2018) and the reasons for their exclusion in the Characteristics of excluded studies table. 
Figure 2.

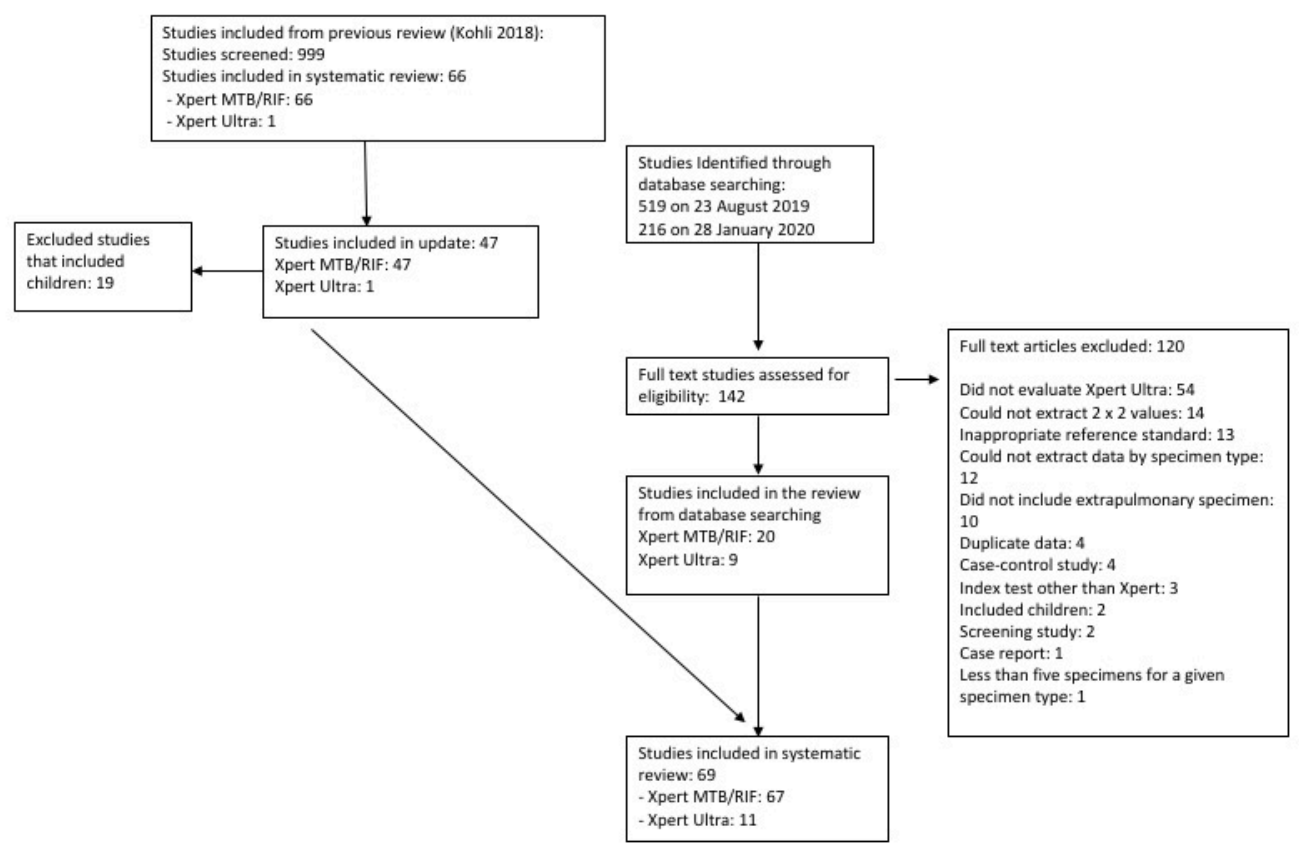

\section{Methodological quality of included studies}

Studies evaluating Xpert MTB/RIF and Xpert Ultra for detection of extrapulmonary tuberculosis

Figure 3 and Figure 4 show risk of bias and applicability concerns for each of the 69 studies included for tuberculosis detection. Risk of bias and applicability concerns are also presented specifically for studies evaluating Xpert Ultra and Xpert MTB/RIF for tuberculous meningitis (Appendix 5), pleural tuberculosis (Appendix 6), and lymph node tuberculosis (Appendix 7).

Figure 3. Risk of bias and applicability concerns graph for tuberculosis detection: review authors' judgements about each domain presented as percentages across included studies.

\begin{tabular}{|c|c|c|c|c|c|c|c|c|c|c|}
\hline Patient Selection & \begin{tabular}{|l|l|}
5 & 11 \\
\end{tabular} & \multicolumn{4}{|c|}{53} & \multicolumn{4}{|c|}{49} & 17 \\
\hline Index Test: Xpert MTB/RIF & \multicolumn{5}{|c|}{67} & & 5 & \multicolumn{3}{|c|}{45} \\
\hline Index Test: Xpert Ultra & \multicolumn{5}{|c|}{11} & 2 & \multicolumn{4}{|c|}{9} \\
\hline Reference Standard & \multicolumn{2}{|c|}{30} & & \multicolumn{2}{|l|}{34} & & \multicolumn{4}{|c|}{53} \\
\hline \multirow[t]{3}{*}{ Flow and Timing } & \multicolumn{5}{|c|}{68} & & & & & \\
\hline & $0 \%$ & $25 \%$ & $50 \%$ & $75 \%$ & $100 \%$ & $0 \%$ & $25 \%$ & $50 \%$ & $75 \%$ & $100 \%$ \\
\hline & \multicolumn{5}{|c|}{ Risk of Bias } & \multicolumn{5}{|c|}{ Applicability Concerns } \\
\hline High & \multicolumn{5}{|c|}{ Unclear } & \multicolumn{4}{|c|}{ Low } & \\
\hline
\end{tabular}


Figure 4. Risk of bias and applicability concerns summary for tuberculosis detection: review authors' judgements about each domain for each included study.

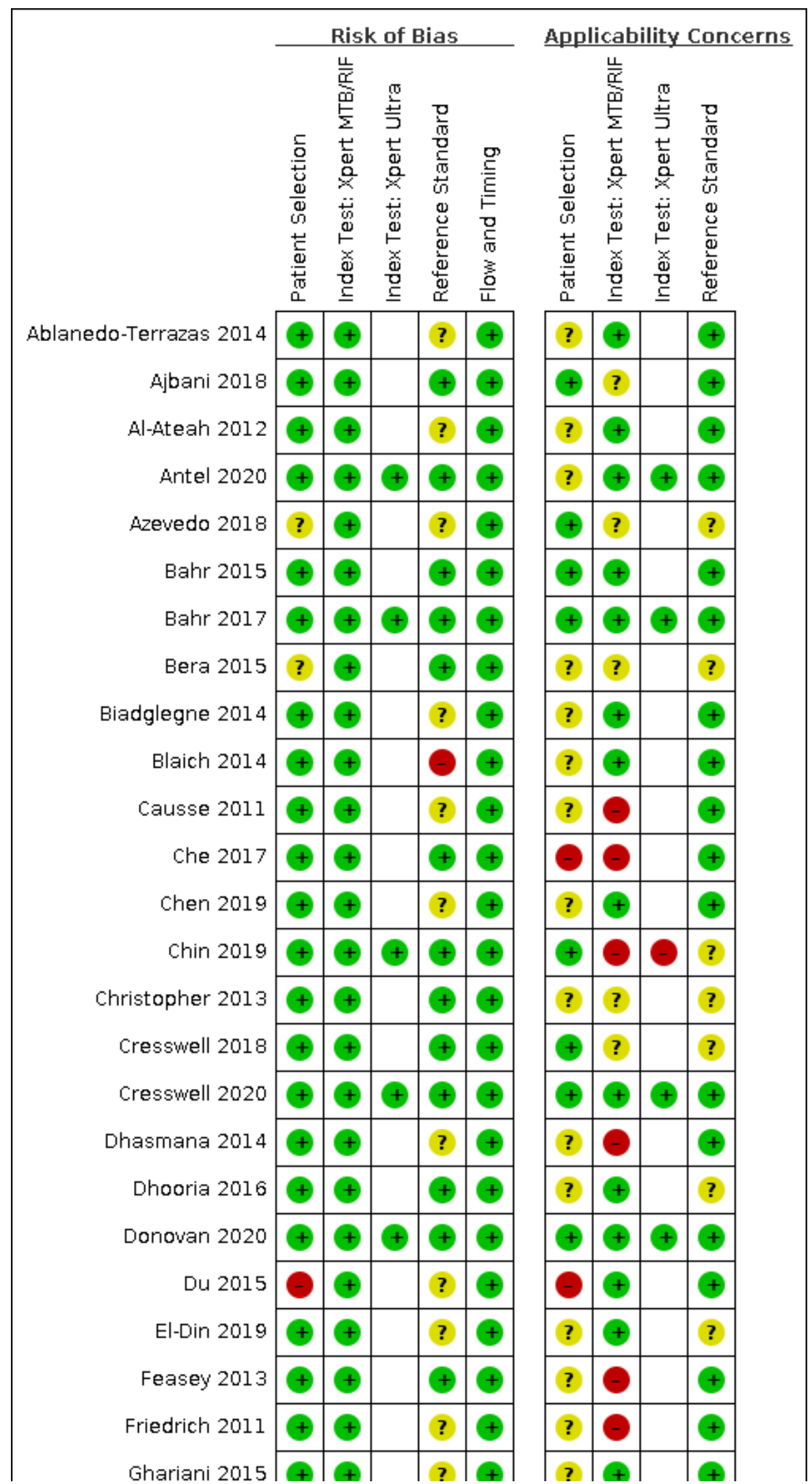


Figure 4. (Continued)

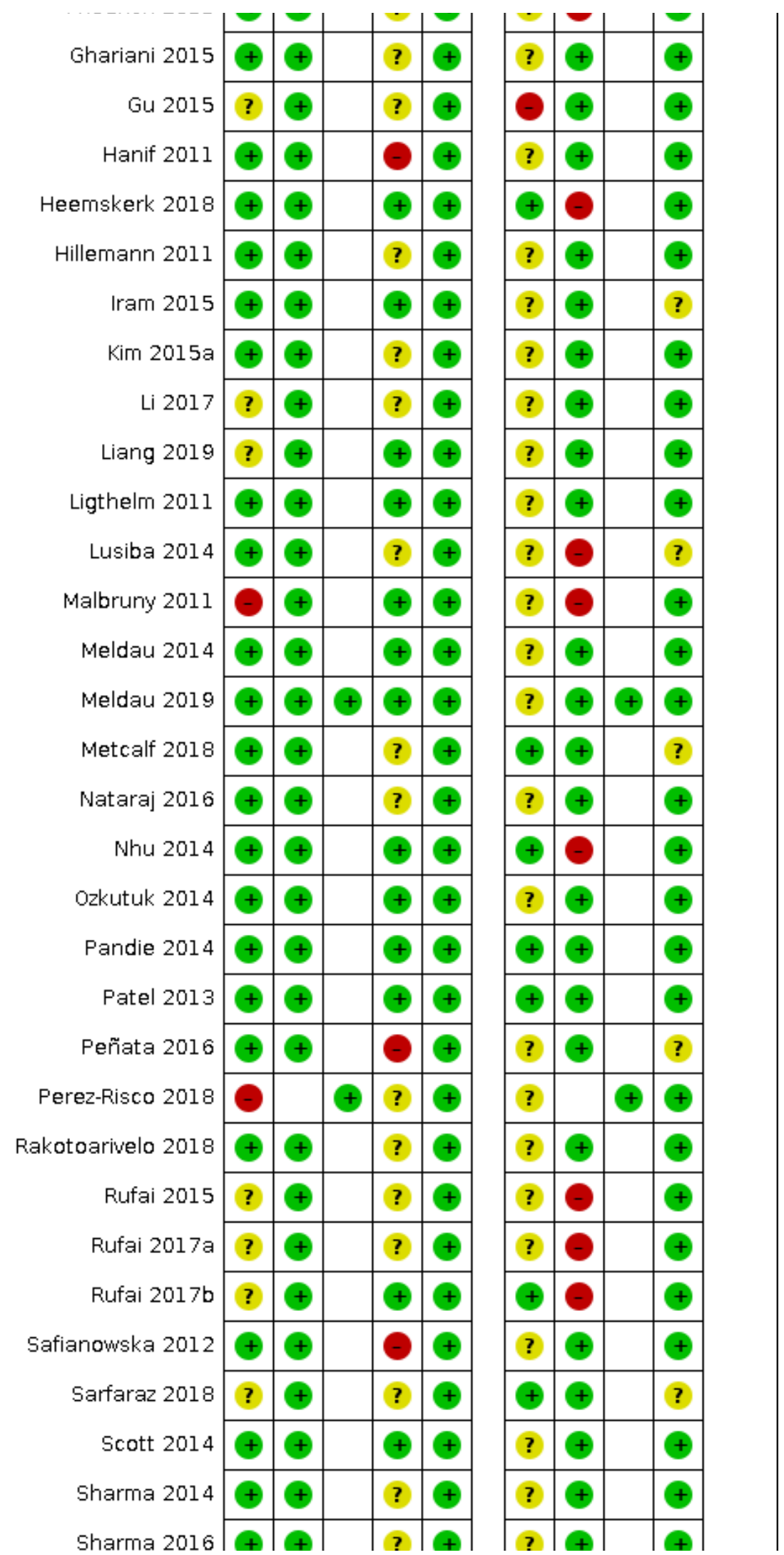


Figure 4. (Continued)

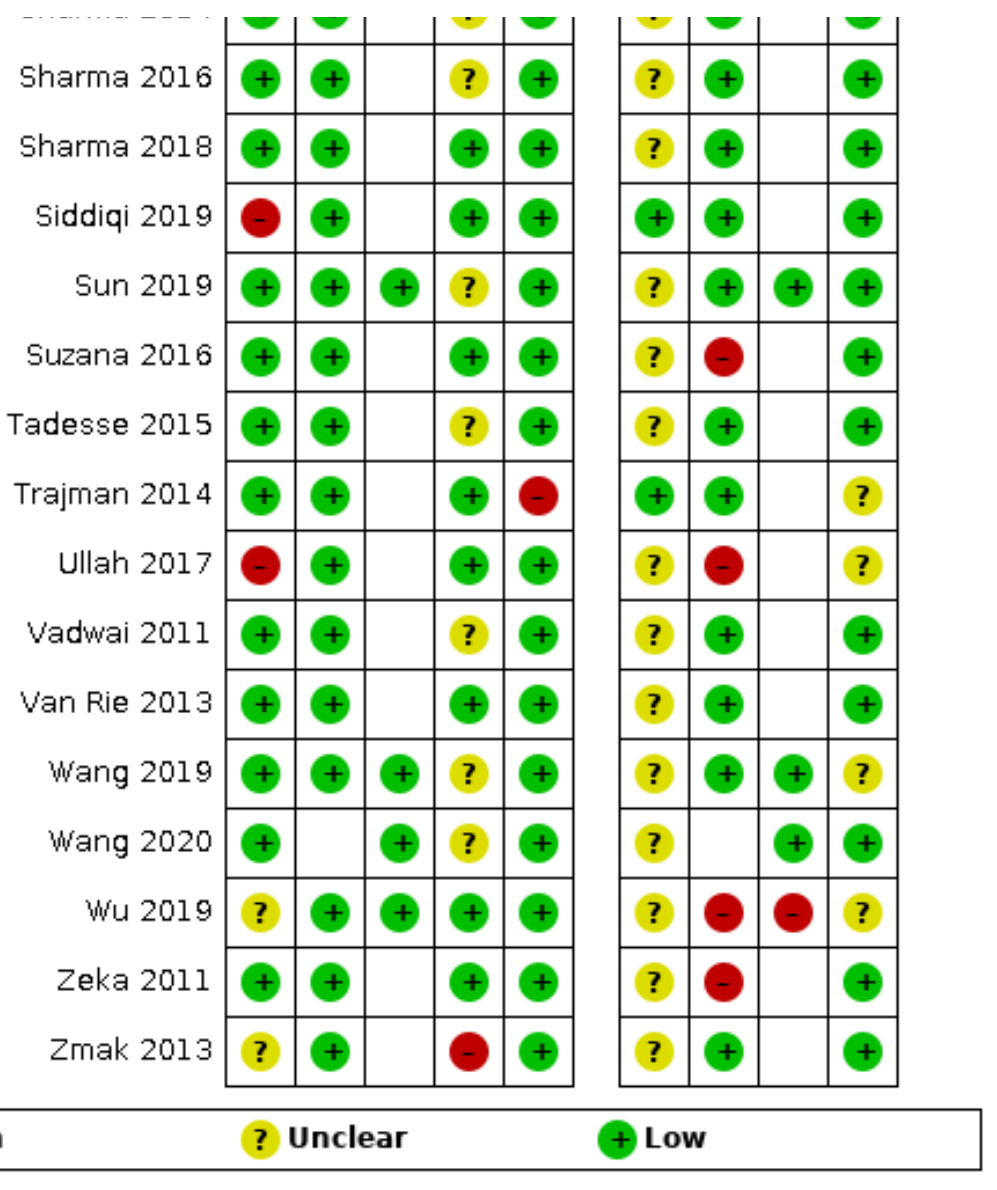

In the patient selection domain, we thought that 53 studies (77\%) had low risk of bias, and five studies (7\%) had high risk of bias for the following reasons: one study selected participants by convenience (Malbruny 2011) and four studies had inappropriate exclusions (Du 2015; Perez-Risco 2018; Siddiqi 2019; Ullah 2017). We thought that 11 studies (16\%) had unclear risk of bias for the following reasons: the manner of patient selection was unclear in ten studies (Azevedo 2018; Gu 2015; Li 2017; Liang 2019; Rufai 2015; Rufai 2017a; Rufai 2017b; Sarfaraz 2018; Wu 2019; Zmak 2013), and it was unclear whether the study avoided inappropriate exclusions: one study (Bera 2015). Regarding applicability (patient characteristics and setting), we thought that 17 studies (25\%) had low concern because participants were evaluated in local hospitals or primary health settings: three studies (Pandie 2014; Sarfaraz 2018; Trajman 2014), or in the case of tuberculous meningitis, tertiary centres: 14 studies (Ajbani 2018; Azevedo 2018; Bahr 2015; Bahr 2017; Chin 2019; Cresswell 2018; Cresswell 2020; Donovan 2020; Heemskerk 2018; Metcalf 2018; Nhu 2014; Patel 2013; Rufai 2017b Siddiqi 2019). Three studies (4\%) had high concern because participants were evaluated exclusively as inpatients at a tertiary care centre (Che 2017; Du 2015; Gu 2015); and 52 (75\%) studies had unclear or high concern because we could not tell the clinical setting or a high percentage of participants had received prior testing for tuberculosis (Antel 2020).

In the index test domain, we thought that all studies had low risk of bias because the results of the index tests (Xpert Ultra and
Xpert MTB/RIF) are automatically generated, the user is provided with printable test results, and the test threshold is prespecified. Regarding applicability, with respect to Xpert Ultra, we thought that 9/11 studies (82\%) had low risk of bias (Antel 2020; Bahr 2017; Cresswell 2020; Donovan 2020; Meldau 2019; Perez-Risco 2018; Sun 2019; Wang 2019; Wang 2020) and 2/11 studies (18\%) had high risk of bias because the index test was not performed according to WHO standard operating procedures (Chin 2019; Wu 2019). With respect to Xpert MTB/RIF, we thought that $45 / 67$ studies (67\%) had low concern because at least $75 \%$ of the specimen types in these studies were processed according to WHO recommendations; $17 / 67$ studies (25\%) had high concern because fewer than $50 \%$ of the specimen types in these studies were processed according to WHO recommendations (Causse 2011; Che 2017; Chin 2019; Dhasmana 2014; Feasey 2013; Friedrich 2011; Heemskerk 2018; Lusiba 2014; Malbruny 2011; Nhu 2014; Rufai 2015; Rufai 2017a; Rufai 2017b; Suzana 2016; Ullah 2017; Wu 2019; Zeka 2011). We thought that $5 / 67$ studies (7\%) had unclear concern because either the manner of specimen processing was not reported (four studies: Ajbani 2018; Azevedo 2018; Bera 2015; Cresswell 2018), or only $50 \%$ of the specimen types were processed according to WHO recommendations (one study: Christopher 2013).

In the reference standard domain, 34 studies (49\%) had low risk of bias because results of the reference standard were interpreted without knowledge of results of the index test and only non-sterile 
specimens were decontaminated (Ajbani 2018; Antel 2020; Bahr 2015; Bahr 2017; Bera 2015; Che 2017; Chin 2019; Christopher 2013 ; Cresswell 2018; Cresswell 2020; Dhooria 2016; Donovan 2020; Feasey 2013; Heemskerk 2018; Iram 2015; Liang 2019; Ligthelm 2011; Malbruny 2011; Meldau 2014; Meldau 2019; Nhu 2014; Ozkutuk 2014; Pandie 2014; Patel 2013; Rufai 2017b; Scott 2014; Sharma 2018; Siddiqi 2019; Suzana 2016; Trajman 2014; Ullah 2017; Van Rie 2013; Wu 2019; Zeka 2011). Five studies (7\%) had high risk of bias because results of the reference standard were interpreted with knowledge of results of the index test (Blaich 2014; Hanif 2011; Peñata 2016; Safianowska 2012; Zmak 2013). Thirty studies $(43 \%)$ had unclear risk of bias for the following reasons: seven studies did not report whether there was blinding of the reference standard (Azevedo 2018; El-Din 2019; Lusiba 2014; Metcalf 2018; Perez-Risco 2018; Wang 2019; Wang 2020); 21 studies decontaminated specimens generally considered to be sterile (AlAteah 2012; Biadglegne 2014; Causse 2011; Chen 2019; Dhasmana 2014; Du 2015; Friedrich 2011; Ghariani 2015; Gu 2015; Hillemann 2011; Kim 2015a; Li 2017; Nataraj 2016; Rakotoarivelo 2018; Rufai 2015; Rufai 2017a; Sharma 2014; Sharma 2016; Sun 2019; Tadesse 2015; Vadwai 2011); and two studies did not report blinding and decontaminated specimens generally considered to be sterile (Ablanedo-Terrazas 2014; Sarfaraz 2018).

Regarding applicability of the reference standard, we thought that 53 studies (77\%) had low concern because these studies performed a test to identify $M$ tuberculosis species (speciation) and 16 studies (23\%) had unclear concern because we could not tell whether the study performed speciation (Azevedo 2018; Bera 2015; Chin 2019; Christopher 2013; Cresswell 2018; Dhooria 2016; El-Din 2019; Iram 2015; Lusiba 2014; Metcalf 2018; Peñata 2016; Sarfaraz 2018; Trajman 2014; Ullah 2017; Wang 2019; Wu 2019).

In the flow and timing domain, we considered almost all studies to have low risk of bias, noting that all participants were accounted for in the analysis. One study included fewer than $50 \%$ of eligible participants in the analysis (Trajman 2014).

\section{Studies evaluating Xpert Ultra for detection of rifampicin resistance}

Figure 5 and Figure 6 show risk-of-bias and applicability concerns for each of the five studies included for rifampicin resistance detection.

Figure 5. Risk of bias and applicability concerns graph for rifampicin resistance detection in comparative studies of Xpert Ultra and Xpert MTB/RIF: review authors' judgements about each domain presented as percentages across included studies.

\begin{tabular}{|c|c|c|c|c|c|c|c|c|c|c|}
\hline \multirow{2}{*}{$\begin{array}{l}\text { Patient Selection } \\
\text { Index Test: Xpert MTB/RIF }\end{array}$} & 1 & \multicolumn{4}{|c|}{4} & \multicolumn{4}{|c|}{4} & 1 \\
\hline & \multicolumn{5}{|c|}{5} & & 2 & \multicolumn{3}{|c|}{3} \\
\hline Index Test: Xpert Ultra & \multicolumn{5}{|c|}{5} & & 2 & \multicolumn{3}{|c|}{3} \\
\hline Reference Standard & \multicolumn{3}{|c|}{3} & 2 & & \multicolumn{3}{|c|}{3} & \multicolumn{2}{|l|}{2} \\
\hline \multirow[t]{3}{*}{ Flow and Timing } & \multicolumn{5}{|c|}{5} & & & & & \\
\hline & $0 \%$ & $25 \%$ & $50 \%$ & $75 \%$ & $100 \%$ & $0 \%$ & $25 \%$ & $50 \%$ & $75 \%$ & $100 \%$ \\
\hline & \multicolumn{5}{|c|}{ Risk of Bias } & \multicolumn{5}{|c|}{ Applicability Concerns } \\
\hline High & \multicolumn{5}{|c|}{ Unclear } & \multicolumn{4}{|c|}{ Low } & \\
\hline
\end{tabular}


Figure 6. Risk of bias and applicability concerns summary for rifampicin resistance detection in comparative studies of Xpert Ultra and Xpert MTB/RIF: review authors' judgements about each domain for each included study.

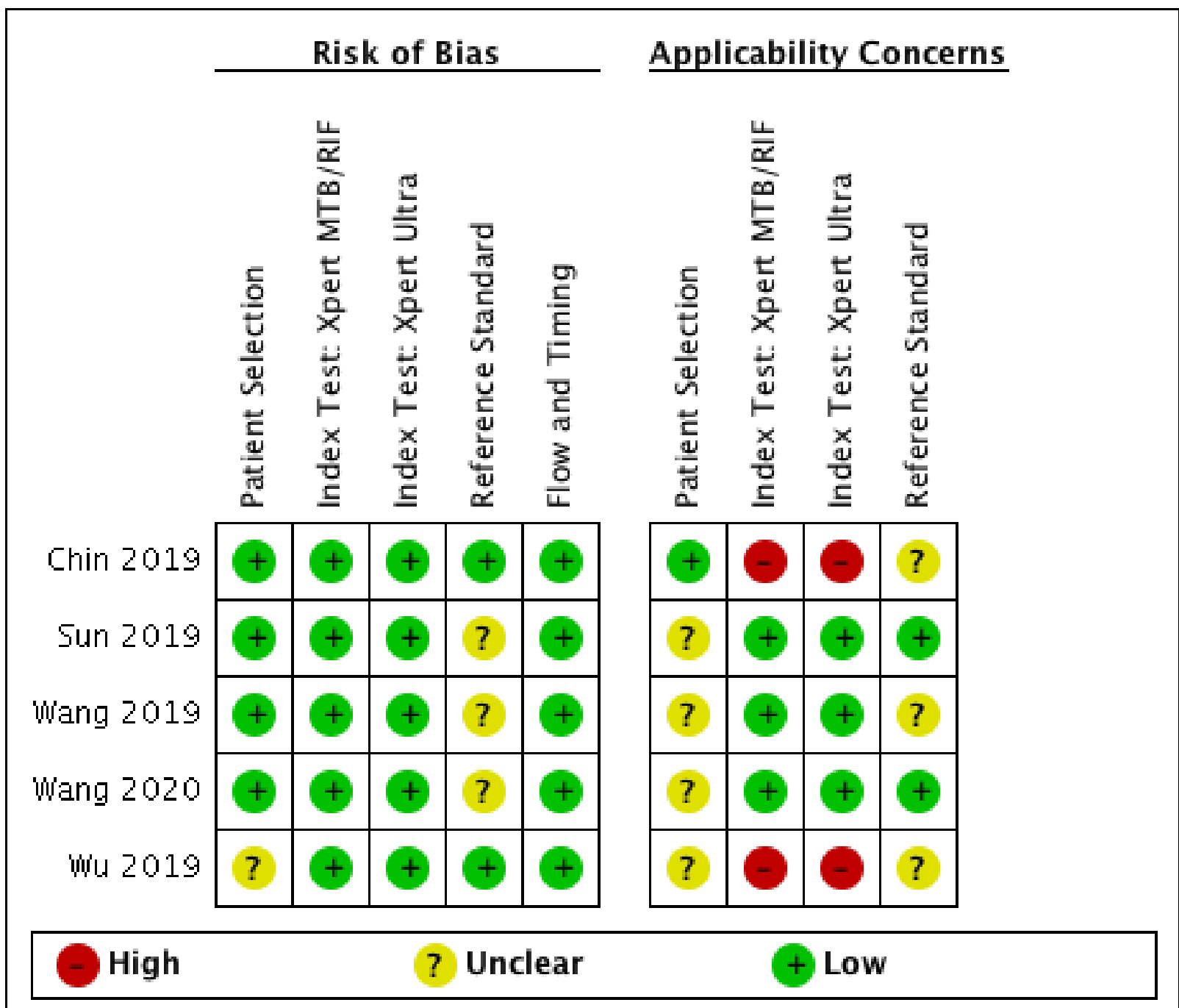

In the patient selection domain, we thought that four studies (80\%) had low risk of bias (Chin 2019; Sun 2019; Wang 2019; Wang 2020) and one study (20\%) had unclear risk of bias as the manner of patient selection was unclear (Wu 2019). We thought that one study $(20 \%)$ had low concern because participants were evaluated exclusively as inpatients at a tertiary care centre (Chin 2019) and four studies $(80 \%)$ had unclear concern because we could not tell the details of the clinical setting (Sun 2019; Wang 2019; Wang 2020; Wu 2019).

In the index test domain, we thought that all studies had low risk of bias because the results of the index tests are automatically generated, the user is provided with printable test results, and the test threshold is prespecified. For applicability, with respect to Xpert Ultra, we thought that three studies (60\%) had low concern because at least $75 \%$ of the specimen types in these studies were processed according to WHO recommendations (Sun 2019; Wang 2019; Wang 2020); two studies (40\%) had high concern because fewer than $50 \%$ of the specimen types in these studies were processed according to WHO recommendations (Chin 2019; Wu 2019).

In the reference standard domain, two studies (40\%) had low risk of bias because results of the reference standard were interpreted without knowledge of results of the index test and only non-sterile specimens were decontaminated (Chin 2019; Wu 2019). Three studies $(60 \%)$ had unclear risk of bias as it was unclear whether blinding of the reference standard was performed (Sun 2019; Wang 2019; Wang 2020). For applicability of the reference standard, we thought that all studies had low concern because detection of rifampicin resistance occurs only when the $M$ tuberculosis target is present within the specimen.

In the flow and timing domain, we considered all studies to have low risk of bias, noting that all participants were accounted for in the analysis. 


\section{Findings}

The 69 studies were conducted in 28 different countries. Most of the studies were conducted in China $(n=10)$, India $(n=13)$, South Africa $(n=10)$, and Uganda $(n=6)$. Seven studies exclusively or mainly included HIV-positive participants (Ablanedo-Terrazas 2014; Azevedo 2018; Bahr 2015; Bahr 2017; Cresswell 2020; Feasey 2013; Van Rie 2013). Most studies performed the index tests and culture on the same specimen type, except for one study in which Xpert MTB/RIF was performed on blood and culture was performed on sputum (Feasey 2013). Most studies did not report the exact number of cultures used to confirm a diagnosis of tuberculosis, but it is likely that many studies used a single culture. We present key characteristics of the included studies in the Characteristics of included studies table.

\section{Detection of extrapulmonary tuberculosis}

Xpert Ultra: of the 11 studies, the number evaluating different specimens was as follows: tuberculous meningitis (CSF) six studies; pleural tuberculosis (pleural fluid) four studies; lymph node tuberculosis (lymph node aspirate) one study; genitourinary tuberculosis (urine) one study; bone or joint tuberculosis (bone or joint aspirate) two studies; and peritoneal tuberculosis (peritoneal fluid) one study.

Xpert MTB/RIF: of the 67 studies, the number of studies evaluating different specimens was as follows: tuberculous meningitis (CSF) 33 studies; pleural tuberculosis (fluid) 27 studies; lymph node tuberculosis (aspirate 15 studies, biopsy 11 studies); genitourinary tuberculosis (urine) 15 studies; bone or joint tuberculosis (aspirate 12 studies, tissue 3 studies); peritoneal tuberculosis (fluid 17 studies, tissue 1 study); pericardial tuberculosis (fluid 14 studies, tissue 2 studies); and disseminated tuberculosis (blood 2 studies). Several studies included more than one type of specimen.

Table 2 presents Xpert Ultra and Xpert MTB/RIF pooled sensitivity and specificity estimates and predictive values by reference standard for all forms of extrapulmonary tuberculosis and specimen types included in the review.

\section{A: Xpert MTB/RIF and Xpert Ultra testing in cerebrospinal fluid for tuberculous meningitis}

\section{Xpert Ultra}

\section{Culture reference standard}

Six studies evaluated Xpert Ultra in cerebrospinal fluid (CSF) specimens against culture (Bahr 2017; Chin 2019; Cresswell 2020; Donovan 2020; Perez-Risco 2018; Wang 2020). Xpert Ultra sensitivity ranged from $80 \%$ to $100 \%$ and specificity ranged from $50 \%$ to $100 \%$ (Figure 7). Chin 2019 reported the lowest specificity (50\%). In this study, the investigators inoculated uncentrifuged CSF which could have led to lower culture positivity, thus resulting in a higher number of false positives. Perez-Risco 2018 (specificity 100\%) contributed only one participant to this analysis. In CSF, Xpert Ultra pooled sensitivity and specificity $(95 \% \mathrm{Crl})$ against culture were $89.4 \%$ ( 79.1 to 95.6 ) and $91.2 \%$ ( 83.2 to 95.7 ), (6 studies; 475 participants, 89 (18.7\%) with tuberculosis); Table 2.

Figure 7. Forest plots of Xpert Ultra sensitivity and specificity in cerebrospinal fluid by reference standard. The squares represent the sensitivity and specificity of one study, the black line its confidence interval. FN: falsenegative; FP: false-positive; TN: true-negative; TP: true-positive.

Cerebrospinal fluid, Xpert Ultra, culture

$\begin{array}{lrrrrrr}\text { Study } & \text { TP } & \text { FP } & \text { FN } & \text { TN } & \text { Sensitivity }(95 \% \mathrm{Cl}) & \text { Specificity }(95 \% \mathrm{Cl}) \text { Sensitivity }(95 \% \mathrm{Cl} \text { Specificity (95\% Cl) } \\ \text { Perez-Risco } 2018 & 3 & 0 & 0 & 1 & 1.00[0.29,1.00] & 1.00[0.03,1.00] \\ \text { Donovan 2020 } & 20 & 4 & 2 & 62 & 0.91[0.71,0.99] & 0.94[0.85,0.98] \\ \text { Bahr 2017 } & 9 & 12 & 1 & 107 & 0.90[0.55,1.00] & 0.90[0.83,0.95] \\ \text { Cresswell 2020 } & 24 & 15 & 3 & 162 & 0.89[0.71,0.98] & 0.92[0.86,0.95] \\ \text { Wang 2019 } & 19 & 0 & 3 & 17 & 0.86[0.65,0.97] & 1.00[0.80,1.00] \\ \text { Chin 2019 } & 4 & 3 & 1 & 3 & 0.80[0.28,0.99] & 0.50[0.12,0.88]\end{array}$

Cerebrospinal fluid, xpert Ultra, composite reference standard

$\begin{array}{lrrrrrr}\text { Study } & \text { TP } & \text { FP } & \text { FN } & \text { TN } & \text { Sensitivity }(95 \% \mathrm{Cl}) & \text { Specificity }(95 \% \mathrm{Cl}) \\ \text { Cresswell 2020 } & 39 & 0 & 12 & 153 & 0.76[0.63,0.87] & 1.00[0.98,1.00] \\ \text { Bahr 2017 } & 16 & 5 & 7 & 101 & 0.70[0.47,0.87] & 0.95[0.89,0.98] \\ \text { Donovan 2020 } & 25 & 0 & 18 & 60 & 0.58[0.42,0.73] & 1.00[0.94,1.00] \\ \text { Wang 2019 } & 19 & 0 & 24 & 17 & 0.44[0.29,0.60] & 1.00[0.80,1.00]\end{array}$

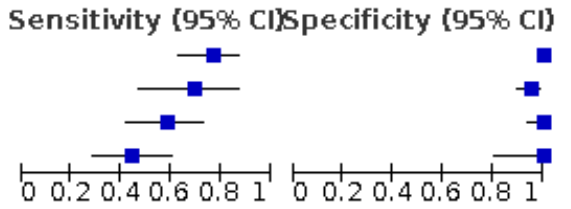

\section{Composite reference standard}

In CSF, Xpert Ultra pooled sensitivity and specificity against a composite reference standard were $62.7 \%$ (45.7 to 77.0$)$ and $99.1 \%$ (96.6 to 99.9), (4 studies; 496 participants); Table 2, Figure 7.

\section{Latent class meta-analysis}

We had insufficient data to obtain robust parameter estimates using the latent class model for Xpert Ultra in CSF. 


\section{Xpert MTB/RIF}

\section{Culture reference standard}

Thirty-three studies evaluated Xpert MTB/RIF in CSF specimens against culture (Ajbani 2018; Al-Ateah 2012; Azevedo 2018; Bahr 2015; Bahr 2017; Blaich 2014; Causse 2011; Chin 2019; Cresswell 2018; Cresswell 2020; Donovan 2020; Hanif 2011; Hillemann 2011; Kim 2015a; Li 2017; Malbruny 2011; Metcalf 2018; Nataraj 2016; Nhu
2014; Ozkutuk 2014; Patel 2013; Peñata 2016; Rakotoarivelo 2018; Rufai 2017b; Safianowska 2012; Sharma 2014; Siddiqi 2019; Suzana 2016; Ullah 2017; Vadwai 2011; Wang 2019; Zeka 2011; Zmak 2013). Xpert MTB/RIF sensitivity ranged from $0 \%$ to $100 \%$ and specificity ranged from $78 \%$ to $100 \%$ (Figure 8 ). For sensitivity, we thought that differences in CSF volume and processing could partly explain the heterogeneity. Three studies (Al-Ateah 2012; Hillemann 2011; Safianowska 2012) did not contribute data to the meta-analysis because sensitivity was not estimable. 
Figure 8. Forest plots of Xpert MTB/RIF sensitivity and specificity in cerebrospinal fluid by reference standard. The squares represent the sensitivity and specificity of one study, the black line its confidence interval. FN: falsenegative; FP: false-positive; TN: true-negative; TP: true-positive.

Cerebrospinal fluid, Xpert MTB/RIF, culture

Study

Al-Ateah 2012

Hillemann 2011

Safianowska 2012

Ajbani 2018

Blaich 2014

Malbruny 2011

Hanif 2011

Pe\& 241:ata 2016

Zeka 2011

Zmak 2013

Metcalf 2018

Nhu 2014

Causse 2011

Patel 2013

Donovan 2020

Li 2017

Kim 2015a

Cresswell 2020

Rakotoarivelo 2018

Sharma 2014

Suzana 2016

Chin 2019

Azevedo 2018

Bahr 2017

Bahr 2015

Rufai $2017 \mathrm{~b}$

Siddiqi 2019

Ullah 2017

Cresswell 2018

Wang 2019

Ozkutuk 2014

Vadwai 2011
Nataraj 2016
TP FP FN TN Sensitivity $(95 \% \mathrm{Cl})$ SP

$\begin{array}{rrrrr}0 & 0 & 0 & 14 & \text { Not estimable } \\ 0 & 0 & 0 & 19 & \text { Not estimable } \\ 0 & 0 & 0 & 6 & \text { Not estimable } \\ 2 & 2 & 0 & 9 & 1.00[0.16,1.00] \\ 2 & 0 & 0 & 2 & 1.00[0.16,1.00] \\ 1 & 0 & 0 & 14 & 1.00[0.03,1.00] \\ 1 & 0 & 0 & 4 & 1.00[0.03,1.00] \\ 6 & 1 & 0 & 148 & 1.00[0.54,1.00] \\ 3 & 0 & 0 & 28 & 1.00[0.29,1.00] \\ 1 & 2 & 0 & 43 & 1.00[0.03,1.00] \\ 35 & 5 & 1 & 119 & 0.97[0.85,1.00] \\ 6 & 1 & 1 & 29 & 0.86[0.42,1.00] \\ 103 & 6 & 18 & 252 & 0.85[0.78,0.91] \\ 5 & 0 & 1 & 44 & 0.83[0.36,1.00] \\ 22 & 1 & 5 & 31 & 0.81[0.62,0.94] \\ 18 & 2 & 5 & 63 & 0.78[0.56,0.93] \\ 3 & 3 & 1 & 67 & 0.75[0.19,0.99] \\ 3 & 0 & 1 & 250 & 0.75[0.19,0.99] \\ 19 & 6 & 8 & 171 & 0.70[0.50,0.86] \\ 9 & 4 & 4 & 60 & 0.69[0.39,0.91] \\ 15 & 3 & 7 & 205 & 0.68[0.45,0.86] \\ 2 & 3 & 1 & 53 & 0.67[0.09,0.99] \\ 3 & 1 & 2 & 5 & 0.60[0.15,0.95] \\ 3 & 0 & 2 & 96 & 0.60[0.15,0.95] \\ 6 & 4 & 4 & 115 & 0.60[0.26,0.88] \\ 7 & 5 & 5 & 63 & 0.58[0.28,0.85] \\ 27 & 11 & 25 & 204 & 0.52[0.38,0.66] \\ 55 & 26 & 52 & 417 & 0.51[0.42,0.61] \\ 2 & 4 & 2 & 22 & 0.50[0.07,0.93] \\ 17 & 17 & 22 & 62 & 0.44[0.28,0.60] \\ 8 & 0 & 14 & 17 & 0.36[0.17,0.59] \\ 1 & 1 & 2 & 107 & 0.33[0.01,0.91] \\ 0 & 0 & 3 & 16 & 0.00[0.00,0.71]\end{array}$

Specificity $(95 \% \mathrm{Cl})$ Sensitivity $(95 \%$ Cl $)$ Specificity $(95 \% \mathrm{Cl})$ $1.00[0.77,1.00]$ $1.00[0.82,1.00]$ $1.00[0.54,1.00]$ $0.82[0.48,0.98]$ $1.00[0.16,1.00]$ $1.00[0.77,1.00]$ $1.00[0.40,1.00]$ $0.99[0.96,1.00]$ $1.00[0.88,1.00]$ $0.96[0.85,0.99]$ $0.96[0.91,0.99]$ $0.97[0.83,1.00]$ $0.98[0.95,0.99]$ $1.00[0.92,1.00]$ $0.97[0.84,1.00]$ $0.97[0.89,1.00]$ $0.96[0.88,0.99]$ $1.00[0.99,1.00]$ $0.97[0.93,0.99]$ $0.94[0.85,0.98]$ $0.99[0.96,1.00]$ $0.95[0.85,0.99]$ $0.83[0.36,1.00]$ $1.00[0.96,1.00]$ $0.97[0.92,0.99]$ $0.93[0.84,0.98]$ $0.95[0.91,0.97]$ $0.94[0.92,0.96]$ $0.85[0.65,0.96]$ $0.78[0.68,0.87]$ $1.00[0.80,1.00]$ $0.99[0.95,1.00]$ $1.00[0.79,1.00]$

Cerebrospinal fluid, Xpert MTB/RIF, composite reference standard

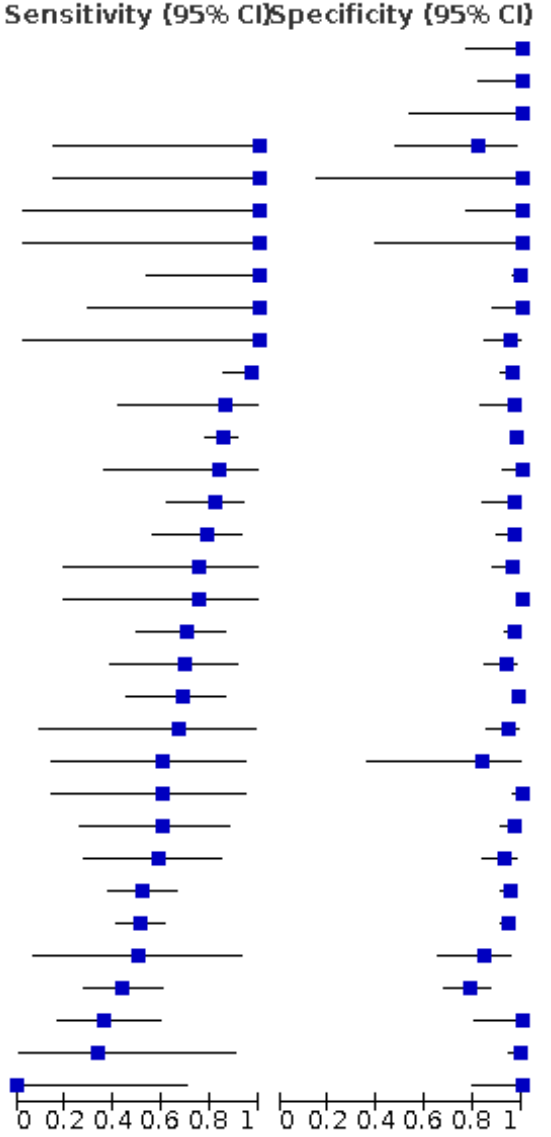

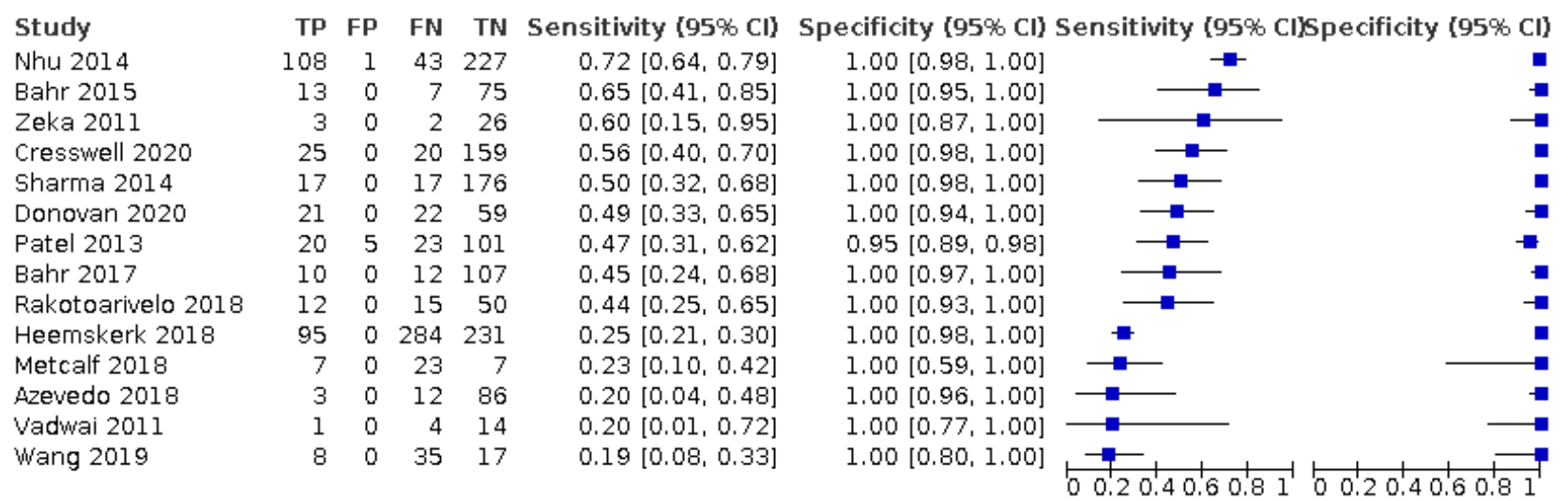

In CSF, Xpert MTB/RIF pooled sensitivity and specificity $(95 \% \mathrm{Crl})$ against culture were $71.1 \%$ (62.8 to 79.1$)$ and $96.9 \%(95.4$ to 98.0), respectively (30 studies; 3395 participants, 571 (16.8\%) with tuberculosis); Table 2, Figure 8.

\section{Composite reference standard}

In CSF, Xpert MTB/RIF pooled sensitivity and specificity against a composite reference standard were $42.3 \%$ (32.1 to 52.8 ) and $99.8 \%$ (99.3 to 100.0), (14 studies; 2203 participants); Table 2, Figure 8. 


\section{Latent class meta-analysis}

Based on the latent class meta-analysis model, Xpert MTB/ RIF pooled sensitivity and specificity $(95 \% \mathrm{Crl})$ for tuberculous meningitis were $74.7 \%$ (65.5 to 84.0 ) and $99.5 \%$ (99.1 to 99.7) (30 studies; 3395 participants); Table 3 . The pooled sensitivity of culture at $80.8 \%$ ( 72.5 to 88.5 ) was estimated to be lower than $100 \%$. The pooled specificity of culture was estimated to be $99.2 \%$ ( 98.7 to 99.5); Table 3.

\section{Xpert Ultra versus Xpert MTB/RIF}

In comparative accuracy studies evaluating both index tests, Xpert Ultra pooled sensitivity and specificity $(95 \% \mathrm{Crl})$ against culture were $89.0 \%$ (77.9 to 95.2$)$ and $91.0 \%$ (82.7 to 95.6$)$ and Xpert MTB/RIF pooled sensitivity and specificity were $62.2 \%$ (43.7 to 78.1 ) and $96.8 \%$ (93.4 to 98.6), (5 studies; 471 participants), direct comparison, Table 2; Figure 9; Figure 10. For CSF, the difference between the sensitivities of Xpert Ultra and Xpert MTB/RIF was $26.2 \%$ (9.1 to 44.4$)$. We estimated the probability that the pooled sensitivity of Xpert Ultra exceeds that of Xpert MTB/RIF was 0.997 . The difference between the specificities of Xpert Ultra and Xpert MTB/RIF was $-5.6 \%(-12.9$ to -0.1$)$. We estimated the probability that the pooled specificity of Xpert Ultra was less than that of Xpert MTB/RIF was 0.978; Table 4.

Figure 9. Forest plots of Xpert Ultra and Xpert MTB/RIF sensitivity and specificity in cerebrospinal fluid, comparative studies. The squares represent the sensitivity and specificity of one study, the black line its confidence interval. FN: false-negative; FP: false-positive; TN: true-negative; TP: true-positive.

Cerebrospinal fluid, xpert Ultra, culture

$\begin{array}{lrrrrr}\text { Study } & \text { TP } & \text { FP } & \text { FN } & \text { TN } & \text { Sensitivity }(95 \% \mathrm{Cl}) \\ \text { Bahr 2017 } & 9 & 12 & 1 & 107 & 0.90[0.55,1.00] \\ \text { Chin 2019 } & 4 & 3 & 1 & 3 & 0.80[0.28,0.99] \\ \text { Cresswell 2020 } & 24 & 15 & 3 & 162 & 0.89[0.71,0.98] \\ \text { Donovan 2020 } & 20 & 4 & 2 & 62 & 0.91[0.71,0.99] \\ \text { Wang 2019 } & 19 & 0 & 3 & 17 & 0.86[0.65,0.97]\end{array}$

Cerebrospinal fluid, Xpert MTB/RIF, culture
Study

Bahr 2017

Chin 2019

Cresswell 2020

Donovan 2020

Wang 2019

$\begin{array}{rrrrr}\text { TP } & \text { FP } & \text { FN } & \text { TN } & \text { Sensitivity }(95 \% \mathrm{Cl}) \\ 6 & 4 & 4 & 115 & 0.60[0.26,0.88] \\ 3 & 1 & 2 & 5 & 0.60[0.15,0.95] \\ 19 & 6 & 8 & 171 & 0.70[0.50,0.86] \\ 18 & 2 & 5 & 63 & 0.78[0.56,0.93] \\ 8 & 0 & 14 & 17 & 0.36[0.17,0.59]\end{array}$

Specificity $(95 \% \mathrm{Cl})$ Sensitivity $(95 \%$ Cl)Specificity $(95 \% \mathrm{Cl})$
$0.90[0.83,0.95]$

$0.50[0.12,0.88]$

$0.92[0.86,0.95]$

$0.94[0.85,0.98]$

$1.00[0.80,1.00]$

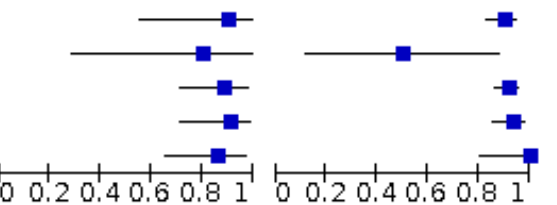

Specificity $(95 \% \mathrm{Cl})$ Sensitivity $(95 \%$ Cl$)$ Specificity $(95 \% \mathrm{Cl})$ $0.97[0.92,0.99]$

$0.83[0.36,1.00]$

$0.97[0.93,0.99]$

$0.97[0.89,1.00]$

$1.00[0.80,1.00]$

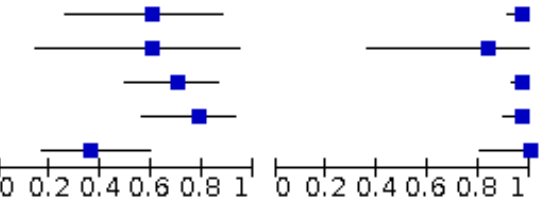


Figure 10. Summary plots of the sensitivity and specificity of Xpert Ultra (A) (5 studies) and Xpert MTB/RIF (B) (5 studies) in cerebrospinal fluid for detection of tuberculous meningitis. Each individual study is represented by a shaded square. The size of the square is proportional to the sample size of the study such that larger studies are represented by larger squares. The filled circle is the median pooled estimate for sensitivity and specificity. The solid curves represent the $95 \%$ credible region around the summary estimate; the dashed curves represent the $95 \%$ prediction region.

(A) Xpert Ultra

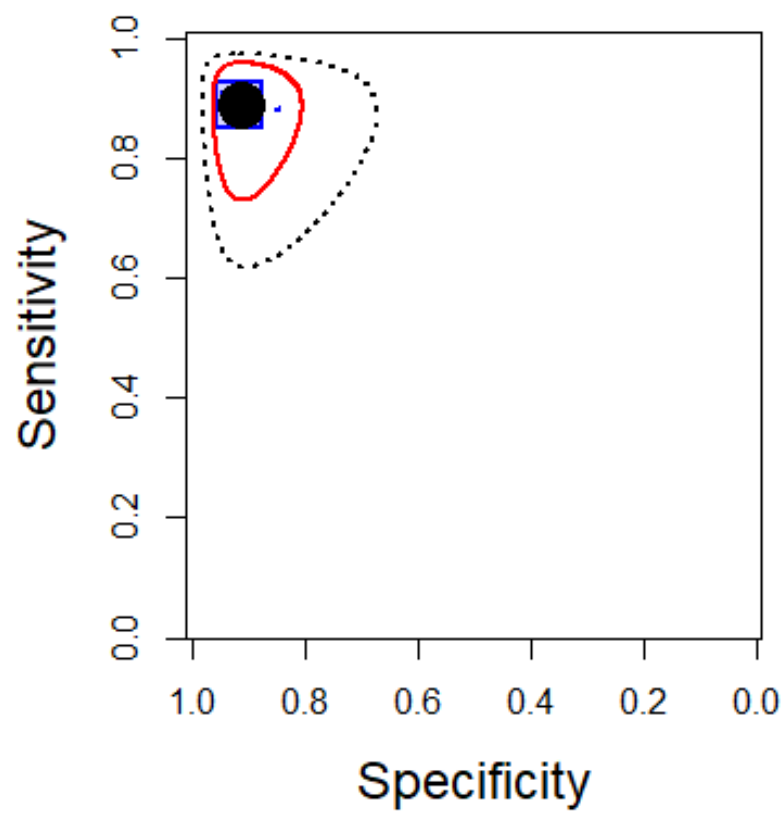

Investigations of heterogeneity

Xpert Ultra versus Xpert MTB/RIF testing in people living with HIV

We identified two studies that directly compared Xpert Ultra and Xpert MTB/RIF, both against culture, in people living with HIV. Sensitivity $(95 \% \mathrm{Cl}$ ) was $90 \%$ (55 to 100$)$ (Bahr 2017) and $89 \%$
(B) Xpert MTB/RIF

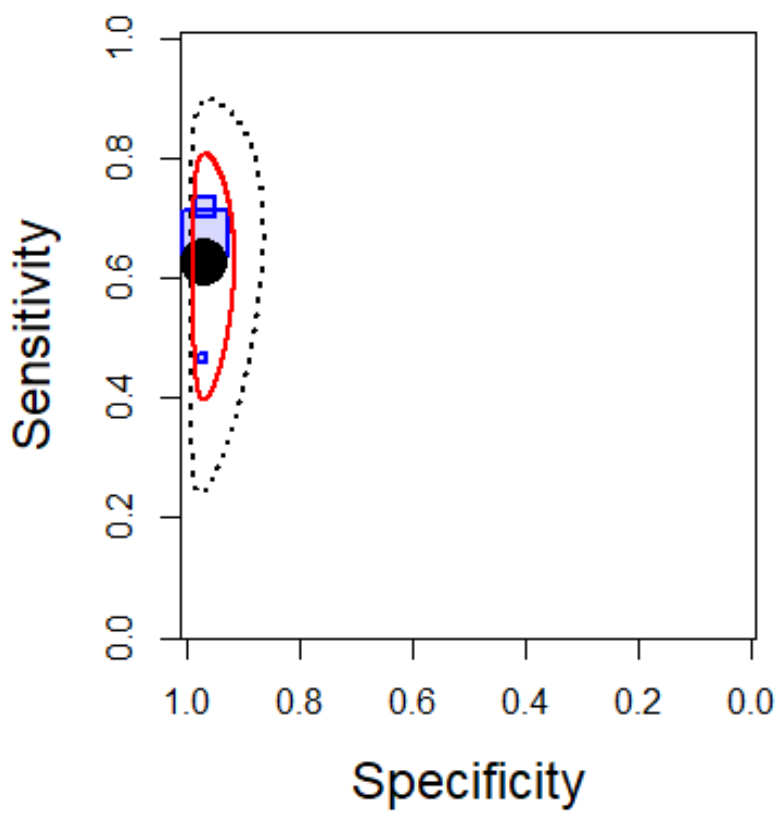

(71 to 98) (Cresswell 2020) for Xpert Ultra and 60\% (26 to 88) (Bahr 2017) and 70\% (50 to 86) (Cresswell 2020) for Xpert MTB/RIF. Specificity $(95 \% \mathrm{Cl}$ ) was $90 \%$ (83 to 95$)$ (Bahr 2017) and 92\% (86 to 95) (Cresswell 2020) for Xpert Ultra and $97 \%$ (95\% Cl 92 to 99) (Bahr 2017) and 97\% (93 to 99) (Cresswell 2020) for Xpert MTB/RIF; Figure 11.

Figure 11. Forest plots of Xpert Ultra and Xpert MTB/RIF sensitivity and specificity in cerebrospinal fluid in HIVpositive people, with respect to culture. The squares represent the sensitivity and specificity of one study, the black line its confidence interval. FN: false-negative; FP: false-positive; TN: true-negative; TP: true-positive. .

Cerebrospinal fluid, Xpert Ultra, HIV positive

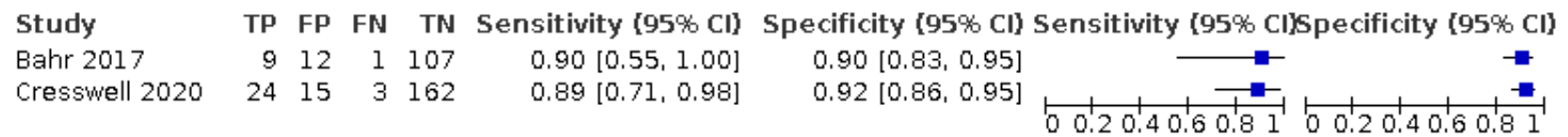

Cerebrospinal fluid, Xpert MTB/RIF, HIV positive

$\begin{array}{lrrrrrr}\text { Study } & \text { TP } & \text { FP } & \text { FN } & \text { TN } & \text { Sensitivity }(95 \% \mathrm{Cl}) & \text { Specificity }(95 \% \mathrm{Cl}) \text { Sensitivity (95\% Cl)Specificity (95\% Cl) } \\ \text { Bahr 2017 } & 6 & 4 & 4 & 115 & 0.60[0.26,0.88] & 0.97[0.92,0.99] \\ \text { Cresswell } 2020 & 19 & 6 & 8 & 171 & 0.70[0.50,0.86] & 0.97[0.93,0.99]\end{array}$




\section{Specimen concentration}

\section{Xpert Ultra}

We found that concentrating CSF improved both Xpert Ultra sensitivity and specificity. Xpert Ultra pooled sensitivity in concentrated specimens was $90.5 \%$ (76.7 to 97.0) (3 studies; 421 participants) versus $88.4 \%$ (67.8 to 97.5 ) (3 studies; 54 participants) in unconcentrated specimens. Xpert Ultra pooled specificity in concentrated specimens was $91.9 \%$ (84.5 to 96.1 ) versus $88.6 \%$ (58.4 to 99.0 ) in unconcentrated specimens; Table 5. The probability that Xpert Ultra sensitivity and specificity are higher with concentrated CSF compared to unconcentrated CSF were 0.630 and 0.653 , respectively.

\section{Xpert MTB/RIF}

We found that concentrating CSF improved both Xpert MTB/ RIF sensitivity and specificity. Xpert MTB/RIF pooled sensitivity in concentrated specimens was $77.6 \%$ (67.2 to 85.9$)(14,2279$ participants) versus $59.4 \%$ (48.3 to 70.5$)$ (17,1123 participants) in unconcentrated specimens. Xpert MTB/RIF pooled specificity in concentrated specimens was $97.4 \%$ (96.1 to 98.4 ) versus $96.8 \%$ (94.0 to 98.7) in unconcentrated specimens, Table 5. The probability that Xpert MTB/RIF sensitivity and specificity are higher with concentrated CSF compared to unconcentrated CSF were 0.989 and 0.696 , respectively.

\section{Cerebrospinal fluid collection volumes}

\section{Xpert Ultra}

Two studies reported the volume of CSF collected for Xpert Ultra testing, $3 \mathrm{~mL}$ in both studies. Sensitivities were similar: 90\% (55 to 100) in Bahr 2017 and 89\% (71 to 98) in Cresswell 2020. Specificities were also similar 90\% (83 to 95) in Bahr 2017 and 92\% (86 to 95) in cresswell 2020.

\section{Impact of tuberculosis prevalence on sensitivity and specificity}

For Xpert Ultra, we found lower sensitivity in settings with higher tuberculosis prevalence (threshold 30\%) than in those with lower tuberculosis prevalence: pooled sensitivity (95\% Crl) of $88.3 \%$ (68.3 to 97.0 ) versus $90.8 \%$ (77.3 to 96.9 ). We found lower specificity in settings with higher tuberculosis prevalence than in those with lower tuberculosis prevalence: pooled specificity of $88.0 \%$ (64.3 to 97.9) versus $91.9 \%$ ( 82.5 to 96.6 ). In both analyses, the $95 \%$ Crls overlapped; Table 6.

Similarly, for Xpert MTB/RIF, we found lower sensitivity in settings with higher tuberculosis prevalence than in those with lower tuberculosis prevalence: pooled sensitivity of $67.0 \%$ (49.0 to 81.5 ) versus $72.0 \%$ (62.4 to 81.2 ). We found lower specificity in settings with higher tuberculosis prevalence than in those with lower tuberculosis prevalence: pooled specificity of $94.1 \%$ (86.8 to 97.9 ) versus $97.3 \%$ (95.9 to 98.3 ). In both analyses, the $95 \%$ Crls overlapped; Table 6 . When we repeated the analysis at lower tuberculosis prevalence (threshold 10\%), in the case of specificity, accuracy in the two groups was significantly different (probability of specificity being lower in the high tuberculosis prevalence group $=0.999)$; Table 6 .

\section{Sensitivity analyses}

Overall, the sensitivity analyses made little difference to the findings; Table 7

\section{Inconclusive Xpert Ultra and Xpert MTB/RIF results}

\section{Xpert Ultra}

None of the studies evaluating Xpert Ultra for tuberculous meningitis reported this information.

\section{Xpert MTB/RIF}

We previously reported that for CSF, of 2096 tests performed, the pooled proportion of inconclusive Xpert MTB/RIF results was $0.9 \%$ (95\% Crl 0.3 to 1.9) (Kohli 2018).

\section{B: Xpert Ultra and Xpert MTB/RIF testing in pleural fluid for pleural tuberculosis}

\section{Xpert Ultra}

\section{Culture reference standard}

Four studies evaluated Xpert Ultra in pleural fluid with respect to culture (Perez-Risco 2018; Wang 2019; Wang 2020; Wu 2019). Xpert Ultra sensitivity ranged from $48 \%$ to $84 \%$ and specificity ranged from $65 \%$ to $100 \%$; Figure 12. 
Figure 12. Forest plots of Xpert Ultra and Xpert MTB/RIF sensitivity and specificity in pleural fluid and tissue by reference standard. The squares represent the sensitivity and specificity of one study, the black line its confidence interval. FN: false-negative; FP: false-positive; TN: true-negative; TP: true-positive.

Pleural fluid, Xpert Ultra, culture

Study

Wang 2020

Wang 2019

Wu 2019

Perez-Risco 2018
TP FP FN TN

$\begin{array}{llll}46 & 1 & 9 & 83\end{array}$

$\begin{array}{llll}48 & 18 & 11 & 33\end{array}$

$\begin{array}{llll}17 & 30 & 6 & 72\end{array}$

Pleural fluid, Xpert Ultra, composite reference standard
(95\% Cl) Specificity $(95 \% \mathrm{Cl})$

$0.84[0.71,0.92]$

$0.81[0.69,0.90]$

$0.74[0.52,0.90]$

$0.48[0.26,0.70]$
$0.99[0.94,1.00]$

$0.65[0.50,0.78]$

$0.71[0.61,0.79]$

$1.00[0.29,1.00]$

$\begin{array}{lrrrrrr}\text { Study } & \text { TP } & \text { FP } & \text { FN } & \text { TN } & \text { Sensitivity }(95 \% \mathrm{Cl}) & \text { Specificity }(95 \% \mathrm{Cl}) \\ \text { Wang 2019 } & 66 & 1 & 42 & 22 & 0.61[0.51,0.70] & 0.96[0.78,1.00] \\ \text { Meldau 2019 } & 18 & 1 & 30 & 83 & 0.38[0.24,0.53] & 0.99[0.94,1.00]\end{array}$

Pleural fluid, Xpert MTB/RIF, culture

$\begin{array}{lrrrrr}\text { Study } & \text { TP } & \text { FP } & \text { FN } & \text { TN } & \text { Sensitivity }[95 \% \text { Cl) } \\ \text { Hillemann 2011 } & 0 & 2 & 0 & 103 & \text { Not estimable } \\ \text { Iram 2015 } & 0 & 0 & 0 & 11 & \text { Not estimable } \\ \text { Christopher 2013 } & 0 & 4 & 0 & 83 & \text { Not estimable } \\ \text { Causse 2011 } & 4 & 0 & 0 & 30 & 1.00[0.40,1.00] \\ \text { Al-Ateah 2012 } & 3 & 0 & 0 & 10 & 1.00[0.29,1.00] \\ \text { Hanif 2011 } & 3 & 0 & 0 & 8 & 1.00[0.29,1.00] \\ \text { Pe\&茾24l;ata 2016 } & 2 & 0 & 0 & 46 & 1.00[0.16,1.00] \\ \text { Nataraj 2016 } & 24 & 3 & 4 & 136 & 0.86[0.67,0.96] \\ \text { Che 2017 } & 12 & 0 & 4 & 62 & 0.75[0.48,0.93] \\ \text { Rakotoarivelo 2018 } & 7 & 0 & 4 & 39 & 0.64[0.31,0.89] \\ \text { Suzana 2016 } & 4 & 4 & 3 & 42 & 0.57[0.18,0.90] \\ \text { Friedrich 2011 } & 5 & 0 & 4 & 15 & 0.56[0.21,0.86] \\ \text { Rufai 2015 } & 23 & 0 & 19 & 119 & 0.55[0.39,0.70] \\ \text { Wang 2020 } & 28 & 1 & 27 & 83 & 0.51[0.37,0.65] \\ \text { Vadwai 2011 } & 5 & 0 & 5 & 19 & 0.50[0.19,0.81] \\ \text { Wu 2019 } & 11 & 3 & 11 & 94 & 0.50[0.28,0.72] \\ \text { Wang 2019 } & 28 & 9 & 29 & 42 & 0.49[0.36,0.63] \\ \text { Scott 2014 } & 59 & 21 & 66 & 336 & 0.47[0.38,0.56] \\ \text { Du 2015 } & 24 & 1 & 31 & 70 & 0.44[0.30,0.58] \\ \text { Sharma 2014 } & 37 & 8 & 54 & 265 & 0.41[0.30,0.51] \\ \text { Ozkutuk 2014 } & 2 & 0 & 3 & 227 & 0.40[0.05,0.85] \\ \text { Li 2017 } & 10 & 18 & 15 & 178 & 0.40[0.21,0.61] \\ \text { Meldau 2014 } & 5 & 6 & 11 & 54 & 0.31[0.11,0.59] \\ \text { Kim 2015a } & 5 & 0 & 44 & 339 & 0.10[0.03,0.22] \\ \text { Malbruny 2011 } & 0 & 0 & 2 & 10 & 0.00[0.00,0.84] \\ \text { Safianowska 2012 } & 0 & 0 & 2 & 30 & 0.00[0.00,0.84] \\ \text { Zmak 2013 } & 0 & 0 & 1 & 41 & 0.00[0.00,0.97] \\ \text { Zeka 2011 } & 0 & 0 & 4 & 52 & 0.00[0.00,0.60]\end{array}$

Specificity $(95 \% \mathrm{Cl})$ Sensitivity $[95 \%$ Cl $\}$ Specificity $(95 \% \mathrm{Cl}$ ) $0.98[0.93,1.00]$

$1.00[0.72,1.00]$ $0.95[0.89,0.99]$ $1.00[0.88,1.00]$ $1.00[0.69,1.00]$ $1.00[0.63,1.00]$ $1.00[0.92,1.00]$ $0.98[0.94,1.00]$ $1.00[0.94,1.00]$ $1.00[0.91,1.00]$ $0.91[0.79,0.98]$ $1.00[0.78,1.00]$ $1.00[0.97,1.00]$ $0.99[0.94,1.00]$ $1.00[0.82,1.00]$ $0.97[0.91,0.99]$ $0.82[0.69,0.92]$ $0.94[0.91,0.96]$ $0.99[0.92,1.00]$ $0.97[0.94,0.99]$ $1.00[0.98,1.00]$ $0.91[0.86,0.94]$ $0.90[0.79,0.96]$ $1.00[0.99,1.00]$ $1.00[0.69,1.00]$ $1.00[0.88,1.00]$ $1.00[0.91,1.00]$ $1.00[0.93,1.00]$

Pleural fluid, Xpert MTB/RIF, composite reference standard

$\begin{array}{lrrrrr}\text { Study } & \text { TP } & \text { FP } & \text { FN } & \text { TN } & \text { Sensitivity }(95 \% \text { Cl) } \\ \text { Wu 2019 } & 37 & 0 & 71 & 23 & 0.34[0.25,0.44] \\ \text { Sharma 2018 } & 16 & 0 & 32 & 30 & 0.33[0.20,0.48] \\ \text { Lusiba 2014 } & 25 & 1 & 62 & 28 & 0.29[0.20,0.39] \\ \text { Meldau 2019 } & 14 & 1 & 35 & 83 & 0.29[0.17,0.43] \\ \text { Friedrich 2011 } & 5 & 0 & 15 & 5 & 0.25[0.09,0.49] \\ \text { Meldau 2014 } & 9 & 1 & 31 & 47 & 0.23[0.11,0.38] \\ \text { Liang 2019 } & 22 & 0 & 133 & 64 & 0.14[0.09,0.21] \\ \text { Christopher 2013 } & 4 & 0 & 26 & 61 & 0.13[0.04,0.31] \\ \text { Trajman 2014 } & 1 & 1 & 32 & 51 & 0.03[0.00,0.16] \\ \text { El-Din 2019 } & 1 & 0 & 45 & 12 & 0.02[0.00,0.12]\end{array}$

Specificity $(95 \% \mathrm{Cl})$ $1.00[0.85,1.00]$ $1.00[0.88,1.00]$ $0.97[0.82,1.00]$ $0.99[0.94,1.00]$ $1.00[0.48,1.00]$ $0.98[0.89,1.00]$ $1.00[0.94,1.00]$ $1.00[0.94,1.00]$ $0.98[0.90,1.00]$ $1.00[0.74,1.00]$

Pleural tissue, Xpert MTB/RIF, culture

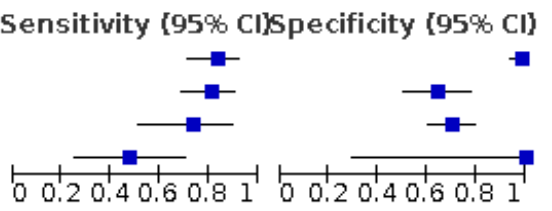

Sensitivity $[95 \%$ Cl]Specificity $[95 \% \mathrm{Cl}]$

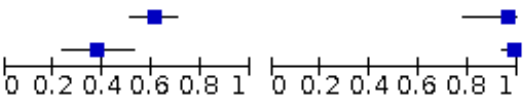

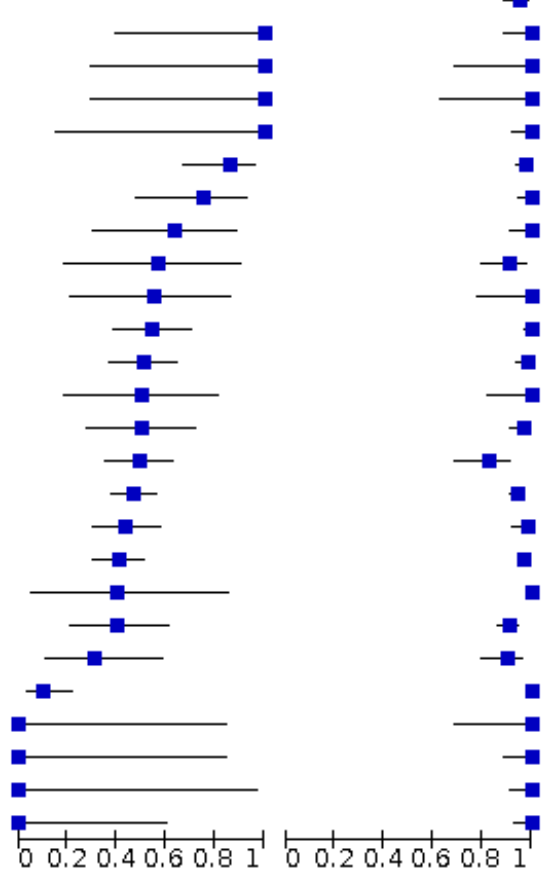

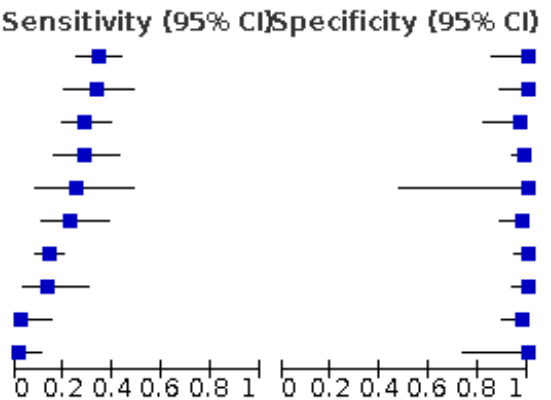

$\begin{array}{lrrrrrr}\text { Study } & \text { TP } & \text { FP } & \text { FN } & \text { TN } & \text { Sensitivity }(95 \% \mathrm{Cl}) & \text { Specificity }(95 \% \mathrm{Cl}) \\ \text { Suzana 2016 } & 0 & 0 & 0 & 7 & \text { Not estimable } & 1.00[0.59,1.00] \\ \text { Du 2015 } & 47 & 2 & 8 & 69 & 0.85[0.73 .0 .941 & 0.97[0.90 .1 .00]\end{array}$

Sensitivity $[95 \%$ Cl]Specificity $(95 \% \mathrm{Cl})$ 
Figure 12. (Continued)

$\begin{array}{lrrrrrr}\text { Suzana 2016 } & 0 & 0 & 0 & 7 & \text { Not estimable } & 1.00[0.59,1.00] \\ \text { Du 2015 } & 47 & 2 & 8 & 69 & 0.85[0.73,0.94] & 0.97[0.90,1.00] \\ \text { Christopher 2013 } & 0 & 1 & 14 & 40 & 0.00[0.00,0.23] & 0.98[0.87,1.00] \\ \text { Ozkutuk 2014 } & 0 & 0 & 2 & 24 & 0.00[0.00,0.84] & 1.00[0.86,1.00]\end{array}$

Pleural tissue, Xpert MTB/RIF, composite reference standard

$\begin{array}{lrrrrrr}\text { Study } & \text { TP } & \text { FP } & \text { FN } & \text { TN } & \text { Sensitivity }(95 \% \mathrm{Cl}) & \text { Specificity }(95 \% \mathrm{Cl}) \\ \text { Christopher } 2013 & 0 & 1 & 14 & 40 & 0.00[0.00,0.23] & 0.98[0.87,1.00]\end{array}$

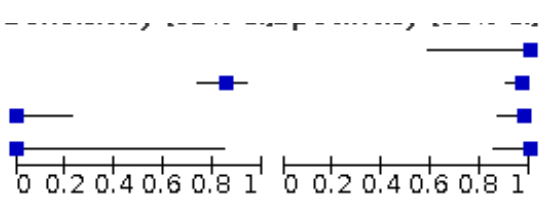

Sensitivity $(95 \%$ Cl)Specificity $(95 \% \mathrm{Cl})$

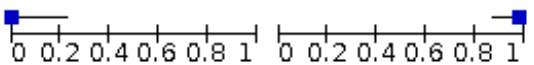

In pleural fluid, Xpert Ultra pooled sensitivity and specificity against culture were $75.0 \%$ (58.0 to 86.4) and $87.0 \%$ (63.1 to 97.9), (4 studies; 398 participants, 158 (39.7\%) with tuberculosis); Table 2; Appendix 8.

\section{Composite reference standard}

Two studies evaluated Xpert Ultra in pleural fluid with respect to a composite reference standard (Meldau 2019; Wang 2019); Figure 12 ; Appendix 8 . Sensitivity ranged from $38 \%$ to $61 \%$, and specificity ranged from $96 \%$ to $99 \%$. We could not explain the variability in the sensitivity estimates and did not perform a meta-analysis.

\section{Latent class meta-analysis}

We had insufficient data to obtain robust parameter estimates using the latent class model for Xpert Ultra in pleural fluid.

\section{Xpert MTB/RIF}

\section{Culture reference standard}

Twenty-eight studies evaluated Xpert MTB/RIF in pleural fluid with respect to culture (Al-Ateah 2012; Causse 2011; Che 2017; Christopher 2013; Du 2015; Friedrich 2011; Hanif 2011; Hillemann 2011; Iram 2015; Kim 2015a; Li 2017; Malbruny 2011; Meldau 2014; Nataraj 2016; Ozkutuk 2014; Peñata 2016; Rakotoarivelo 2018; Rufai 2015; Safianowska 2012; Scott 2014; Sharma 2014; Suzana 2016; Vadwai 2011; Wang 2019; Wang 2020; Wu 2019; Zeka 2011; Zmak 2013). Xpert MTB/RIF sensitivity ranged from $0 \%$ to $100 \%$ and specificity ranged from $82 \%$ to $100 \%$ (Figure 12). Three studies (Christopher 2013; Hillemann 2011; Iram 2015) did not contribute data to the meta-analysis because sensitivity was not estimable.

In pleural fluid, Xpert MTB/RIF pooled sensitivity and specificity against culture were $49.5 \%$ (39.8 to 59.9 ) and $98.9 \%$ (97.6 to 99.7 ) (25 studies; 3065 participants, 644 (21.0\%) with tuberculosis); Table 2; Appendix 8.

\section{Composite reference standard}

In pleural fluid, Xpert MTB/RIF pooled sensitivity and specificity against a composite reference standard were $18.9 \%$ (11.5 to 27.9 ) and 99.3\% (98.1 to 99.8), (10 studies; 1024 participants) Table 2; Figure 12.

\section{Latent class meta-analysis}

Based on the latent class meta-analysis model, Xpert MTB/RIF pooled sensitivity and specificity $(95 \% \mathrm{Crl})$ in pleural fluid were
$53.1 \%$ (42.8 to 64.1 ) and $99.6 \%$ (99.3 to 99.8 ) (25 studies; 3065 participants) Table 3. Xpert MTB/RIF pooled sensitivity was slightly higher and its pooled specificity comparable to what was obtained when culture was treated as having perfect accuracy, with pooled sensitivity of $49.5 \%$ (39.8 to 59.9 ) and pooled specificity of $98.8 \%$ (97.6 to 99.7). The pooled sensitivity of culture at $89.5 \%$ (80.5 to 96.3) was estimated to be lower than $100 \%$. The pooled specificity of culture was estimated to be $99.0 \%$ (98.2 to 99.5 ).

\section{Xpert Ultra versus Xpert MTB/RIF}

We had insufficient data for this analysis.

\section{Impact of tuberculosis prevalence on sensitivity and specificity}

For Xpert Ultra, we had insufficient data for this analysis.

For Xpert MTB/RIF, we found higher sensitivity in settings with higher tuberculosis prevalence than in those with lower tuberculosis prevalence: pooled sensitivity ( $95 \% \mathrm{Crl}$ ) of $20.7 \%$ (11.2 to 33.7 ) versus $15.5 \%$ (6.5 to 30.1 ). We found similar specificity in settings with higher tuberculosis prevalence and in those with lower tuberculosis prevalence: pooled specificity of $99.6 \%$ (97.9 to 99.9) versus $99.0 \%$ (96.9 to 99.8). In both analyses, the $95 \%$ Crls overlapped; Table 6.

\section{Sensitivity analyses}

For Xpert Ultra, we had insufficient data for these analyses.

\section{Inconclusive Xpert Ultra and Xpert MTB/RIF results}

\section{Xpert Ultra}

Of the total 1013 tests performed, the percentage of inconclusive Xpert Ultra results was $0.3 \%$. Only one study reported this information (Wang 2019).

\section{Xpert MTB/RIF}

We previously reported that for pleural fluid, of 1416 tests performed the pooled proportion of inconclusive Xpert MTB/RIF results was $1.2 \%$ (95\% Crl 0.4 to 2.6) (Kohli 2018). 
C: Xpert Ultra and Xpert MTB/RIF testing in pleural tissue for pleural tuberculosis

\section{Xpert Ultra}

\section{Culture reference standard}

We did not identify any studies evaluating Xpert Ultra in pleural tissue against culture.

\section{Composite reference standard}

We did not identify any studies evaluating Xpert Ultra in pleural tissue against a composite reference standard.

\section{Xpert MTB/RIF}

Culture reference standard

Four studies evaluated Xpert MTB/RIF in pleural tissue with respect to culture (Christopher 2013; Du 2015; Ozkutuk 2014; Suzana 2016).
Xpert MTB/RIF sensitivity ranged from $0 \%$ to $85 \%$ and specificity ranged from $97 \%$ to $100 \%$; Figure 12 . One study reported zero tuberculosis cases (Suzana 2016). We did not perform a metaanalysis.

\section{Composite reference standard}

In pleural tissue, Xpert MTB/RIF sensitivity and specificity against a composite reference standard were $0 \%$ (0 to 23 ) and $98 \%$ ( 87 to 100 ) (1 study; 55 participants; Christopher 2013); Figure 12.

D: Xpert MTB/RIF and Xpert Ultra testing in lymph node aspirate for lymph node tuberculosis

Xpert Ultra

\section{Culture reference standard}

In lymph node aspirates, Xpert Ultra sensitivity and specificity against culture were $78 \%$ (40 to 97 ) and $78 \%$ (66 to 87 ), (1 study; 73 participants; 9 (12.3\%) with tuberculosis; Antel 2020); Figure 13.

Figure 13. Forest plots of Xpert Ultra and Xpert MTB/RIF sensitivity and specificity in lymph node aspirate by reference standard. The squares represent the sensitivity and specificity of one study, the black line its confidence interval. FN: false-negative; FP: false-positive; TN: true-negative; TP: true-positive.

Lymph node aspirate, Xpert Ultra, culture

Study TP FP FN TN Sensitivity $(95 \% \mathrm{Cl})$ Specificity $(95 \% \mathrm{Cl})$

Antel $2020 \quad 7 \quad 14 \quad 2 \quad 50 \quad 0.78[0.40,0.97] \quad 0.78[0.66,0.87]$

Lymph node aspirate, Xpert Ultra, composite reference standard

Sensitivity $[95 \%$ Cl]Specificity $(95 \% \mathrm{Cl})$ $\stackrel{1}{1} \stackrel{1}{1} \frac{1}{0.20 .40 .60 .81}$

Study TP FP FN TN Sensitivity $(95 \% \mathrm{Cl})$ Specificity $(95 \% \mathrm{Cl})$

Antel $2020 \quad 21 \quad 0 \quad 9 \quad 43 \quad 0.70[0.51,0.85] \quad 1.00[0.92,1.00]$

Sensitivity $[95 \%$ Cl $)$ Specificity $(95 \% \mathrm{Cl})$

Lymph node aspirate, Xpert MTB/RIF, culture

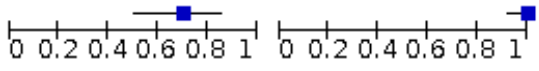

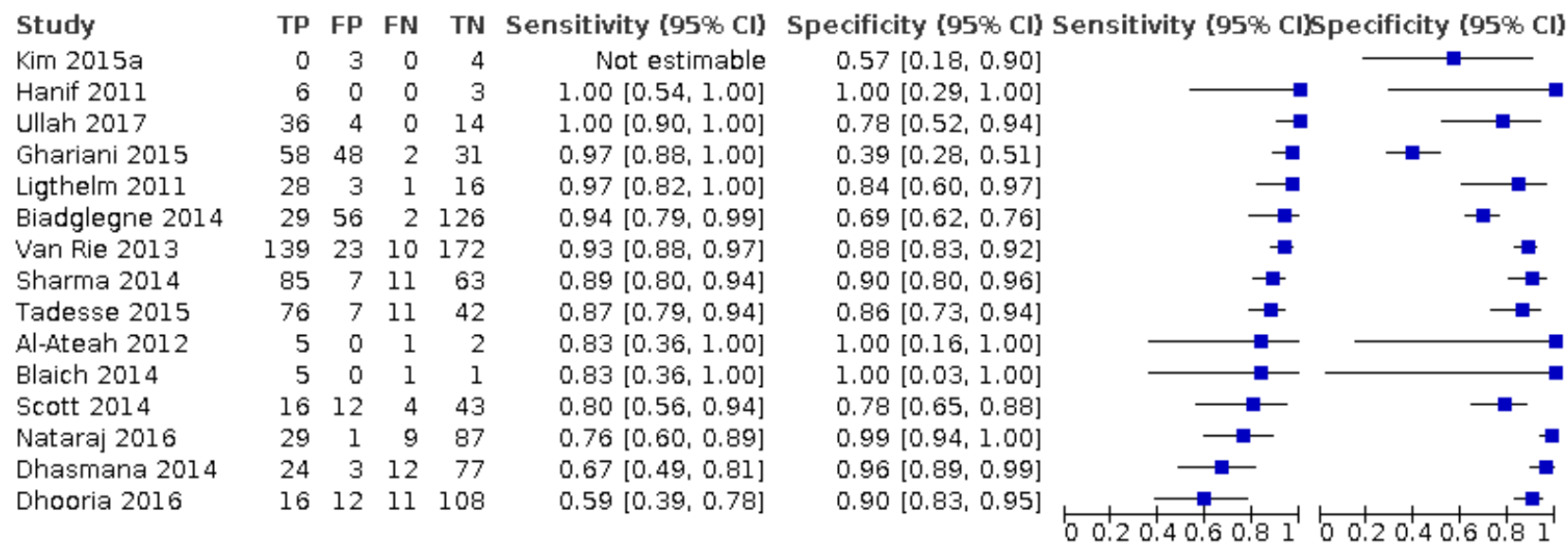

Lymph node aspirate, Xpert MTB/RIF, composite reference standard

Study TP FP FN TN Sensitivity $(95 \% \mathrm{Cl})$ Specificity $(95 \% \mathrm{Cl})$

Ligthelm $2011 \quad 29 \quad 2 \quad 1 \quad 16 \quad 0.97[0.83,1.00] \quad 0.89[0.65,0.99]$

Tadesse $2015 \quad 81 \quad 4 \quad 11 \quad 40 \quad 0.88[0.80,0.94] \quad 0.91[0.78,0.97]$

$\begin{array}{lllllll}\text { Van Rie } 2013 & 160 & 2 & 42 & 144 & 0.79[0.73,0.85] & 0.99[0.95,1.00]\end{array}$

$\begin{array}{lrrrrr}\text { Dhooria } 2016 & 26 & 2 & 27 & 92 & 0.49\end{array}[0.35,0.63] \quad 0.98[0.93,1.00]$

Sensitivity $(95 \%$ Cl]Specificity $(95 \% \mathrm{Cl})$

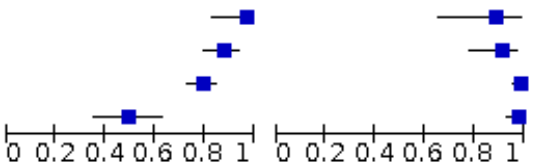




\section{Composite reference standard}

In lymph node aspirates, Xpert Ultra sensitivity and specificity against a composite reference standard were $70 \%$ (51 to 85) and $100 \%$ (92 to 100$)$, (1 study; 73 participants; 9 (12.3\%) with tuberculosis; Antel 2020); Figure 13. Of note, with a composite reference standard, specificity was higher $(100 \%)$ than that observed when using culture as the reference standard $(78 \%)$.

\section{Latent class meta-analysis}

We had insufficient data to obtain robust parameter estimates using the latent class model for Xpert Ultra in lymph node aspirate.

\section{Xpert MTB/RIF}

\section{Culture reference standard}

Fifteen studies evaluated Xpert MTB/RIF in lymph node aspirates with for culture (Al-Ateah 2012; Biadglegne 2014; Blaich 2014; Dhasmana 2014; Dhooria 2016; Ghariani 2015; Hanif 2011; Kim 2015a; Ligthelm 2011; Nataraj 2016; Scott 2014; Sharma 2014; Tadesse 2015; Ullah 2017; Van Rie 2013). Xpert MTB/RIF sensitivity ranged from $59 \%$ to $100 \%$ and specificity from $57 \%$ to $100 \%$; Figure 13. Xpert MTB/RIF specificity in lymph node aspirates was considerably more heterogeneous than in CSF and pleural fluid. The variability in Xpert MTB/RIF specificity in lymph node aspirates was unexpected and may be the result of a systematic, unexplained bias in some studies. One study did not contribute data to the metaanalysis because sensitivity was not estimable (Kim 2015a).

In lymph node aspirates, Xpert MTB/RIF pooled sensitivity and specificity against culture were $88.9 \%(82.7$ to 93.6$)$ and $86.2 \%$ (78.0 to 92.3) (14 studies; 1588 participants, 627 (39.5\%) with tuberculosis); Table 2.

\section{Composite reference standard}

In lymph node aspirates, Xpert MTB/RIF pooled sensitivity and specificity against a composite reference standard were 81.6\% (61.9 to 93.3 ) and $96.4 \%$ (91.3 to 98.6), (4 studies; 679 participants); Table 2; Figure 13. Of note, with a composite reference standard, specificity was less variable and pooled specificity higher than that observed when using culture as the reference standard (86.0\%).

\section{Latent class meta-analysis}

Based on the latent class meta-analysis model, Xpert MTB/RIF pooled sensitivity and specificity $(95 \% \mathrm{Crl})$ in lymph node aspirate were $91.3 \%$ (84.9 to 96.3) and 99.5\% (99.1 to 99.7) (14 studies; 1588 participants); Table 3. Xpert MTB/RIF pooled sensitivity and pooled specificity were higher than when culture was treated as having perfect accuracy, with pooled sensitivity of $88.9 \%$ (82.7 to 93.6 ) and pooled specificity of $86.2 \%$ ( 78.0 to 92.3 ). The pooled sensitivity of culture at $84.9 \%$ ( 74.0 to 92.8 ) was estimated to be lower than $100 \%$. The pooled specificity of culture was estimated to be $98.8 \%(97.7$ to 99.4); Table 3. The latent class meta-analysis resulted in high precision in the specificity of Xpert MTB/RIF across studies. This was the result of adjustments for the imperfect and heterogeneous accuracy of culture across studies.

\section{Xpert Ultra versus Xpert MTB/RIF}

We had insufficient data for this analysis.

Impact of tuberculosis prevalence on sensitivity and specificity

For Xpert Ultra, we had insufficient data for this analysis.

For Xpert MTB/RIF, we found higher sensitivity in settings with higher tuberculosis prevalence than in those with lower tuberculosis prevalence: pooled sensitivity $(95 \% \mathrm{Crl})$ of $93.1 \%$ (88.9 to 96.3 ) versus $72.2 \%$ (64.9 to 87.2 ). We found lower specificity in settings with higher tuberculosis prevalence than in those with lower tuberculosis prevalence: pooled specificity of $83.2 \%(69.5$ to 92.1 ) versus $90.0 \%$ (78.3 to 95.9 ). In the case of sensitivity, accuracy in the two groups was significantly different (probability of sensitivity being lower in the high tuberculosis prevalence group $=0.999)$; Table 6 .

\section{Sensitivity analyses}

For Xpert Ultra, we had insufficient data for these analyses.

\section{Inconclusive Xpert MTB/RIF and Xpert Ultra results}

Xpert Ultra

None of the studies reported this information.

\section{Xpert MTB/RIF}

We previously reported that for lymph node aspirates, in the 1134 tests performed, the pooled proportion of inconclusive Xpert MTB/ RIF results was $1.0 \%$ (95\% Crl 0.4 to 2.0 ) (Kohli 2018).

\section{E: Xpert MTB/RIF and Xpert Ultra in lymph node biopsies for lymph node tuberculosis}

\section{Xpert Ultra}

\section{Culture reference standard}

In lymph node biopsies, Xpert Ultra sensitivity and specificity against culture were $90 \%$ (55 to 100) and $87 \%$ (77 to 94) (Antel 2020) and $100 \%$ (75 to 100$)$ and $38 \%$ (22 to 55) (Wu 2019), (2 studies; 131 participants, 23 (17.6\%) with tuberculosis); Figure 14. 
Figure 14. Forest plots of Xpert Ultra and Xpert MTB/RIFsensitivity and specificity in lymph node biopsy by reference standard. The squares represent the sensitivity and specificity of one study, the black line its confidence interval. FN: false-negative; FP: false-positive; TN: true-negative; TP: true-positive.

Lymph node biopsy, Xpert Ultra, culture

$\begin{array}{lrrrrrr}\text { Study } & \text { TP } & \text { FP } & \text { FN } & \text { TN } & \text { Sensitivity }(95 \% \mathrm{Cl}) & \text { Specificity }[95 \% \mathrm{Cl}) \\ \text { Wu 2019 } & 13 & 23 & 0 & 14 & 1.00[0.75,1.00] & 0.38[0.22,0.55] \\ \text { Antel } 2020 & 9 & 9 & 1 & 62 & 0.90[0.55,1.00] & 0.87[0.77,0.94]\end{array}$

Lymph node biopsy, Xpert Ultra, composite reference standard

Study TP FP FN TN Sensitivity $(95 \% \mathrm{Cl})$ Specificity $(95 \% \mathrm{Cl})$

Antel $2020 \quad 16 \quad 2 \quad 6 \quad 55 \quad 0.73[0.50,0.89] \quad 0.96[0.88,1.00]$

Lymph node biopsy, Xpert MTB/RIF, culture

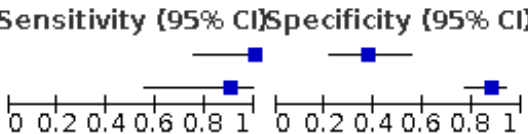

Sensitivity (95\% Cl)Specificity $(95 \% \mathrm{Cl})$

Lymph node biopsy, Xpert MTB/RIF, composite

\begin{abstract}
$1.00[0.29,1.00]$
$0.95[0.75,1.00]$

$0.94[0.71,1.00]$

$0.89[0.67,0.99]$

$0.88[0.75,0.95]$

$0.79[0.49,0.95]$

$0.79[0.49,0.95]$

$0.75[0.19,0.99]$

$0.71[0.29,0.96]$

$0.66[0.53,0.77]$

$0.50[0.12,0.88]$
\end{abstract}

Specificity $(95 \% \mathrm{Cl})$ Sensitivity $(95 \%$ Cl $)$ Specificity $(95 \% \mathrm{Cl})$ $0.00[0.00,0.84]$ $0.59[0.43,0.73]$ $1.00[0.95,1.00]$ $0.31[0.11,0.59]$ $0.93[0.83,0.98]$ $0.83[0.52,0.98]$ $0.53[0.36,0.69]$ $0.67[0.09,0.99]$ $0.92[0.83,0.97]$ $0.80[0.74,0.86]$ $0.93[0.81,0.99]$

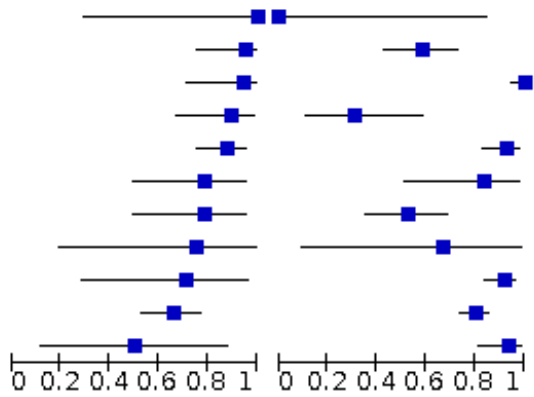

Sensitivity (95\% CI)Specificity (95\% Cl)

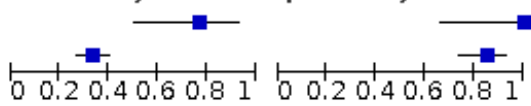

\section{Composite reference standard}

In lymph node biopsies, Xpert Ultra sensitivity and specificity against a composite reference standard were $73 \%$ (50 to 89 ) and 96\% (88 to 100) (Antel 2020), (1 study; 81 participants); Figure 14.

\section{Xpert MTB/RIF}

\section{Culture reference standard}

Eleven studies evaluated Xpert MTB/RIF in lymph node biopsies against culture (Blaich 2014; Causse 2011; Ghariani 2015; Kim 2015a; Ozkutuk 2014; Peñata 2016; Sarfaraz 2018; Sharma 2014; Suzana 2016; Wu 2019; Zeka 2011). Xpert MTB/RIF sensitivity ranged from $50 \%$ to $100 \%$ and specificity ranged from $0 \%$ to $100 \%$; Figure 14 . We could not explain the heterogeneity in accuracy estimates by study quality, small numbers, or other factors.

In lymph node biopsies, Xpert MTB/RIF pooled sensitivity and specificity against culture were $82.4 \%$ (73.5 to 89.7 ) and $80.3 \%$
(60.3 to 91.5), (11 studies; 786 participants, 220 (28.0\%) with tuberculosis); Table 2.

\section{Composite reference standard}

In lymph node biopsies, Xpert MTB/RIF sensitivity and specificity against a composite reference standard were $33 \%$ (27 to 40 ) and $85 \%$ ( 73 to 93 ) (Sarfaraz 2018) and $76 \%$ (50 to 93) and specificity of $100 \%$ (66 to 100) (Zeka 2011) (2 studies; 288 participants); Figure 14.

\section{F: Xpert Ultra and Xpert MTB/RIF testing in urine for genitourinary tuberculosis}

\section{Xpert Ultra}

\section{Culture reference standard}

In urine, Xpert Ultra sensitivity and specificity against culture were $100 \%$ (74 to 100 ) and 100\% (74 to 100), (1 study; 24 participants, 12 (50\%) with tuberculosis) (Perez-Risco 2018); Figure 15. 
Figure 15. Forest plots of Xpert MTB/RIF and Xpert Ultra sensitivity and specificity in urine by reference standard. The squares represent the sensitivity and specificity of one study, the black line its confidence interval. FN: falsenegative; FP: false-positive; TN: true-negative; TP: true-positive.

Urine, Xpert Ultra, culture

Study TP FP FN TN Sensitivity $(95 \% \mathrm{Cl})$ Specificity $(95 \% \mathrm{Cl})$ Perez-Risco $2018 \quad 12 \quad 0 \quad 0 \quad 12 \quad 1.00[0.74,1.00] \quad 1.00[0.74,1.00]$

Sensitivity $(95 \%$ Cl)Specificity $(95 \% \mathrm{Cl})$

Urine, Xpert MTB/RIF, culture

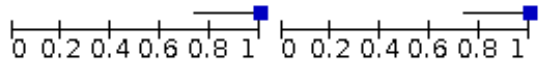

$\begin{array}{lrrrrr}\text { Study } & \text { TP } & \text { FP } & \text { FN } & \text { TN } & \text { Sensitivity }(95 \% \text { Cl) } \\ \text { Causse 2011 } & 0 & 0 & 0 & 58 & \text { Not estimable } \\ \text { Nataraj 2016 } & 0 & 0 & 0 & 12 & \text { Not estimable } \\ \text { Safianowska 2012 } & 0 & 0 & 0 & 1 & \text { Not estimable } \\ \text { Malbruny 2011 } & 0 & 2 & 0 & 1 & \text { Not estimable } \\ \text { Zmak 2013 } & 0 & 0 & 0 & 50 & \text { Not estimable } \\ \text { Blaich 2014 } & 1 & 0 & 0 & 0 & 1.00[0.03,1.00] \\ \text { Hanif 2011 } & 1 & 0 & 0 & 1 & 1.00[0.03,1.00] \\ \text { Kim 2015a } & 4 & 1 & 0 & 101 & 1.00[0.40,1.00] \\ \text { Hillemann 2011 } & 5 & 1 & 0 & 70 & 1.00[0.48,1.00] \\ \text { Suzana 2016 } & 2 & 2 & 0 & 3 & 1.00[0.16,1.00] \\ \text { Chen 2019 } & 34 & 28 & 2 & 238 & 0.94[0.81,0.99] \\ \text { Ozkutuk 2014 } & 9 & 0 & 3 & 329 & 0.75[0.43,0.95] \\ \text { Li 2017 } & 6 & 3 & 2 & 19 & 0.75[0.35,0.97] \\ \text { Sharma 2014 } & 1 & 0 & 2 & 52 & 0.33[0.01,0.91] \\ \text { Zeka 2011 } & 0 & 0 & 1 & 23 & 0.00[0.00,0.97]\end{array}$

Specificity (95\% Cl) Sensitivity $(95 \%$ Cl)Specificity $(95 \% \mathrm{Cl})$ $1.00[0.94,1.00]$

$1.00[0.74,1.00]$

$1.00[0.03,1.00]$

$0.33[0.01,0.91]$

$1.00[0.93,1.00]$ Not estimable

$1.00[0.03,1.00]$

$0.99[0.95,1.00]$

$0.99[0.92,1.00]$

$0.60[0.15,0.95]$

$0.89[0.85,0.93]$

$1.00[0.99,1.00]$

$0.86[0.65,0.97]$

$1.00[0.93,1.00]$

$1.00[0.85,1.00]$

Urine, Xpert MTB/RIF, composite reference standard

$\begin{array}{lrrrrrr}\text { Study } & \text { TP } & \text { FP } & \text { FN } & \text { TN } & \text { Sensitivity }(95 \% \mathrm{Cl}) & \text { Specificity }(95 \% \mathrm{Cl}) \\ \text { Chen 2019 } & 62 & 0 & 88 & 266 & 0.41[0.33,0.50] & 1.00[0.99,1.00] \\ \text { Sharma 2014 } & 1 & 0 & 2 & 44 & 0.33[0.01,0.91] & 1.00[0.92,1.00]\end{array}$

Sensitivity $(95 \%$ Cl)Specificity $(95 \% \mathrm{Cl})$

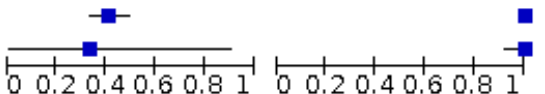

\section{Composite reference standard}

We did not identify any studies that evaluated Xpert Ultra in urine against a composite reference standard.

\section{Xpert MTB/RIF}

\section{Culture reference standard}

Fifteen studies evaluated Xpert MTB/RIF in urine against culture (Blaich 2014; Causse 2011; Chen 2019; Hanif 2011; Hillemann 2011; Kim 2015a; Li 2017; Malbruny 2011; Nataraj 2016; Ozkutuk 2014; Safianowska 2012; Sharma 2014; Suzana 2016; Zeka 2011; Zmak 2013). Xpert MTB/RIF sensitivity ranged from $0 \%$ to $100 \%$ (sensitivity of $0 \%$ was reported by Zeka 2011 that had only one culture positive, which was Xpert negative) and specificity from $33 \%$ to $100 \%$ (Figure 15). Six studies (Blaich 2014; Causse 2011; Malbruny 2011; Nataraj 2016; Sharma 2014; Zmak 2013) did not contribute data to the meta-analysis because either sensitivity or specificity was not estimable.

In urine, Xpert MTB/RIF pooled sensitivity and specificity against culture were $85.9 \%$ (71.4 to 94.3 ) and $98.1 \%$ (93.1 to 99.7) (9 studies; 943 participants, 72 (7.6\%) with tuberculosis); Table 2.

\section{Composite reference standard}

In urine, Xpert MTB/RIF sensitivity and specificity against a composite reference standard were 33\% (1 to 91) and 100\% (92 to
100) (Sharma 2014), and $41 \%$ (33 to 50 ) and $100 \%$ (99 to 100) (Chen 2019) (2 studies; 463 participants); Figure 15.

\section{G: Xpert Ultra and Xpert MTB/RIF testing in bone or joint aspirate for bone or joint tuberculosis}

Xpert Ultra

\section{Culture reference standard}

In bone or joint aspirate, Xpert Ultra sensitivity and specificity against culture were $88 \%$ (47 to 100) (specificity was not estimable) (Perez-Risco 2018), and 96\% (87 to 100) and 97\% (85 to 100) (Sun 2019) (2 studies; 94 participants, 60 (63.8\%) with tuberculosis); Appendix 9.

\section{Composite reference standard}

In bone or joint aspirate, Xpert Ultra sensitivity and specificity against a composite reference standard were $96 \%$ (91 to 99) and 97\% (85 to 100), (1 study; 145 participants; Sun 2019); Appendix 9.

\section{Xpert MTB/RIF}

\section{Culture reference standard}

Twelve studies evaluated Xpert MTB/RIF in bone or joint fluid for culture (Al-Ateah 2012; Blaich 2014; Gu 2015; Kim 2015a; Li 2017; Malbruny 2011; Nataraj 2016; Ozkutuk 2014; Peñata 2016; Safianowska 2012; Sun 2019; Suzana 2016). Xpert MTB/RIF 
sensitivity ranged from $96 \%$ to $100 \%$ and specificity ranged from $53 \%$ to $100 \%$; Appendix 9 .

In bone or joint aspirate, Xpert MTB/RIF pooled sensitivity and specificity against culture were $97.9 \%$ (93.1 to 99.6$)$ and $97.4 \%$ (80.2 to 100.0); (6 studies; 471 participants, 110 (23.4\%) with tuberculosis); Table 2

\section{Composite reference standard}

In bone or joint aspirate, Xpert MTB/RIF sensitivity and specificity against a composite reference standard were $82 \%$ (69 to 91) and $100 \%$ (69 to 100) (Gu 2015), and $94 \%$ ( 87 to 97 ) and $100 \%$ (90 to 100) (Sun 2019); (2 studies; 205 participants); Appendix 9.

\section{H: Xpert Ultra and Xpert MTB/RIF testing in tissue for bone or joint tuberculosis}

\section{Xpert Ultra}

\section{Culture reference standard}

We did not identify any studies that evaluated Xpert Ultra in tissue for bone or joint tuberculosis against culture.

\section{Composite reference standard}

We did not identify any studies that evaluated Xpert Ultra in tissue for bone or joint tuberculosis against a composite reference standard.

\section{Xpert MTB/RIF}

\section{Culture reference standard}

Three studies evaluated Xpert MTB/RIF in bone or joint tissue against culture. Xpert MTB/RIF sensitivity ranged from $50 \%$ to $100 \%$ and specificity ranged from $94 \%$ to $100 \%$ (Appendix 9).

In bone or joint tissue, Xpert MTB/RIF sensitivity and specificity $(95 \% \mathrm{Cl}$ ) against culture were $100 \%$ (3 to 100$)$ and $100 \%$ (48 to 100 )
(Malbruny 2011), 100\% (3 to 100) and 100\% (40 to 100) (Ozkutuk 2014), and 50\% (1 to 99) and 94\% (71 to 100) (Peñata 2016); (3 studies; 30 participants, 4 (13.3\%) with tuberculosis).

\section{Composite reference standard}

We did not identify any studies that evaluated Xpert MTB/RIF in tissue for bone or joint tuberculosis against a composite reference standard.

\section{$\mathrm{J}$ : Xpert Ultra and Xpert MTB/RIF testing in peritoneal fluid for peritoneal tuberculosis}

\section{Xpert Ultra}

\section{Culture reference standard}

In peritoneal fluid, Xpert Ultra sensitivity against culture was 33\% (1 to 91 ) and specificity was not estimable (Perez-Risco 2018) (1 study; 3 participants); Appendix 10.

\section{Composite reference standard}

We did not identify any studies that evaluated Xpert Ultra in peritoneal fluid against a composite reference standard.

\section{Xpert MTB/RIF}

\section{Culture reference standard}

Seventeen studies evaluated Xpert MTB/RIF in peritoneal fluid against culture (Al-Ateah 2012; Causse 2011; Iram 2015; Kim 2015a; Li 2017; Malbruny 2011; Ozkutuk 2014; Peñata 2016; Rufai 2017a; Safianowska 2012; Scott 2014; Sharma 2014; Suzana 2016; Ullah 2017; Vadwai 2011; Zeka 2011; Zmak 2013). Four studies (Al-Ateah 2012; Causse 2011; Iram 2015; Safianowska 2012) did not contribute data to the meta-analysis because sensitivity was not estimable. In individual studies, Xpert MTB/RIF sensitivity ranged from $33 \%$ to $100 \%$ and specificity ranged from $90 \%$ to $100 \%$; Figure 16 ; Appendix 10. 
Figure 16. Forest plots of Xpert MTB/RIF sensitivity and specificity for peritoneal TB (fluid and tissue) by reference standard. The squares represent the sensitivity and specificity of one study, the black line its confidence interval. FN: false-negative; FP: false-positive; TN: true-negative; TP: true-positive.

Peritoneal fluid, Xpert Ultra, culture

Study

Perez-Risco 2018
TP FP FN TN Sensit

Peritoneal fluid, Xpert MTB/RIF, culture
Sensitivity $[95 \%$ Cl $)$ Specificity $(95 \% \mathrm{Cl})$

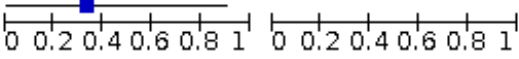

$\begin{array}{lrrrrr}\text { Study } & \text { TP } & \text { FP } & \text { FN } & \text { TN } & \text { Sensitivity }(95 \% \text { Cl) } \\ \text { Safianowska 2012 } & 0 & 0 & 0 & 8 & \text { Not estimable } \\ \text { Iram 2015 } & 0 & 0 & 0 & 7 & \text { Not estimable } \\ \text { Causse 2011 } & 0 & 0 & 0 & 20 & \text { Not estimable } \\ \text { Al-Ateah 2012 } & 0 & 0 & 0 & 4 & \text { Not estimable } \\ \text { Ullah 2017 } & 4 & 4 & 0 & 48 & 1.00[0.40,1.00] \\ \text { Suzana 2016 } & 2 & 2 & 0 & 12 & 1.00[0.16,1.00] \\ \text { Pe\&\#241;ata 2016 } & 1 & 0 & 0 & 14 & 1.00[0.03,1.00] \\ \text { Vadwai 2011 } & 2 & 0 & 0 & 9 & 1.00[0.16,1.00] \\ \text { Malbruny 2011 } & 1 & 0 & 0 & 2 & 1.00[0.03,1.00] \\ \text { Li 2017 } & 3 & 2 & 1 & 48 & 0.75[0.19,0.99] \\ \text { Rufai 2017a } & 12 & 0 & 5 & 50 & 0.71[0.44,0.90] \\ \text { Scott 2014 } & 19 & 3 & 13 & 104 & 0.59[0.41,0.76] \\ \text { Kim 2015a } & 4 & 0 & 5 & 50 & 0.44[0.14,0.79] \\ \text { Zmak 2013 } & 1 & 0 & 2 & 7 & 0.33[0.01,0.91] \\ \text { Sharma 2014 } & 3 & 1 & 13 & 85 & 0.19[0.04,0.46] \\ \text { Ozkutuk 2014 } & 0 & 0 & 2 & 40 & 0.00[0.00,0.84] \\ \text { Zeka 2011 } & 0 & 1 & 1 & 4 & 0.00[0.00,0.97]\end{array}$

Peritoneal tissue, Xpert MTB/RIF, culture

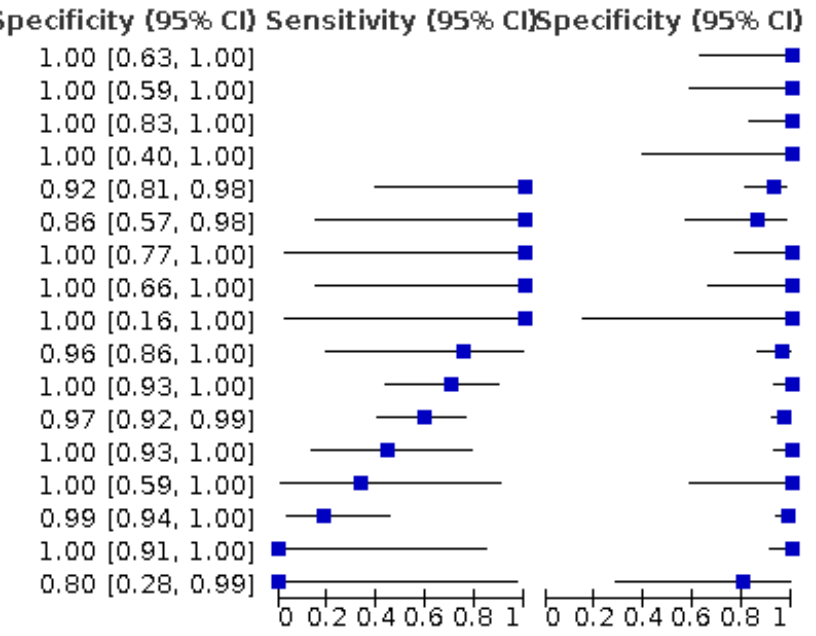

(95\% Cl) Sensitivity (95\% Cl)Specific $1.00[0.40,1.00]$ $0.92[0.81,0.98]$ $0.86[0.57,0.98]$ $1.00[0.77,1.00]$ $1.00[0.66,1.00]$ $1.00[0.16,1.00]$ $0.96[0.86,1.00]$ $1.00[0.93,1.00]$ $0.97[0.92,0.99]$ $1.00[0.93,1.00]$ $1.00[0.59,1.00]$ $.99[0.94,1.00]$ $0.80[0.28,0.99]$

Sensitivity $(95 \%$ Cl)Specificity $(95 \% \mathrm{Cl}$

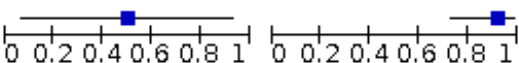

Pericardial fluid, Xpert MTB/RIF, culture

\begin{tabular}{|c|c|c|c|c|c|c|c|}
\hline Study & TP & FP & FN & TN & Sensitivity $(95 \% \mathrm{Cl})$ & Specificity $(95 \% \mathrm{Cl})$ & Sensitivity $(95 \%$ Cl)Specificity $(95 \% \mathrm{Cl}$ \\
\hline Al-Ateah 2012 & 0 & 0 & 0 & 3 & Not estimable & $1.00[0.29,1.00]$ & \\
\hline Safianowska 2012 & 0 & 0 & 0 & 1 & Not estimable & $1.00[0.03,1.00]$ & \\
\hline Pe\&\#241; ata 2016 & 0 & 0 & 0 & 2 & Not estimable & $1.00[0.16,1.00]$ & \\
\hline Kim 2015a & 0 & 0 & 0 & 22 & Not estimable & $1.00[0.85,1.00]$ & \\
\hline Causse 2011 & 0 & 0 & 0 & 12 & Not estimable & $1.00[0.74,1.00]$ & \\
\hline Ozkutuk 2014 & 0 & 0 & 0 & 18 & Not estimable & $1.00[0.81,1.00]$ & \\
\hline Vadwai 2011 & 0 & 0 & 0 & 1 & Not estimable & $1.00[0.03,1.00]$ & \\
\hline Zmak 2013 & 0 & 0 & 0 & 17 & Not estimable & $1.00[0.80,1.00]$ & \\
\hline Blaich 2014 & 1 & 0 & 0 & 0 & $1.00[0.03,1.00]$ & Not estimable & \\
\hline Ullah 2017 & 4 & 0 & 0 & 12 & $1.00[0.40,1.00]$ & $1.00[0.74,1.00]$ & \\
\hline Zeka 2011 & 1 & 0 & 0 & 5 & $1.00[0.03,1.00]$ & $1.00[0.48,1.00]$ & \\
\hline Pandie 2014 & 28 & 27 & 19 & 60 & $0.60[0.44,0.74]$ & $0.69[0.58,0.78]$ & $\rightarrow-$ \\
\hline Sharma 2014 & 1 & 1 & 3 & 15 & $0.25[0.01,0.81]$ & $0.94[0.70,1.00]$ & \\
\hline Suzana 2016 & 0 & 0 & 1 & 4 & $0.00[0.00,0.97]$ & $1.00[0.40,1.00]$ & \\
\hline
\end{tabular}

Pericardial fluid, Xpert MTB/RIF, composite reference standard

Study TP FP FN TN Sensitivity $(95 \% \mathrm{Cl})$ Specificity $(95 \% \mathrm{Cl})$ $\begin{array}{llllllll}\text { Pandie } 2014 & 41 & 0 & 14 & 5 & 0.75[0.61,0.85] & 1.00 & {[0.48,1.00]}\end{array}$ Sharma $2014 \quad 2 \quad 0 \quad 3 \quad 12 \quad 0.40[0.05,0.85] \quad 1.00[0.74,1.00]$

Blood, Xpert MTB/RIF, culture

$\begin{array}{lrrrcrr}\text { Study } & \text { TP } & \text { FP } & \text { FN } & \text { TN } & \text { Sensitivity }(95 \% \text { CI) } & \text { Specificity }(95 \% \mathrm{Cl}) \\ \text { Zmak 2013 } & 0 & 0 & 0 & 11 & \text { Not estimable } & 1.00[0.72,1.00] \\ \text { Feasey } 2013 & 5 & 4 & 4 & 61 & 0.56[0.21,0.86] & 0.94[0.85,0.98]\end{array}$

Sensitivity (95\% Cl)Specificity (95\% Cl)

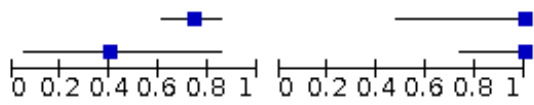

Sensitivity $(95 \%$ Cl)Specificity $(95 \% \mathrm{Cl})$ 00.20.40.60.81 
In peritoneal fluid, Xpert MTB/RIF pooled sensitivity and specificity against culture were $59.1 \%$ (42.1 to 76.2 ) and $97.6 \%$ (95.4 to 98.9 ),

(13 studies; 580 participants, 94 (16.2\%) with tuberculosis); Table 2.

\section{Composite reference standard}

We did not identify any studies that evaluated Xpert MTB/RIF in peritoneal fluid against a composite reference standard.

\section{K: Xpert Ultra and Xpert MTB/RIF testing in tissue for peritoneal tuberculosis}

\section{Xpert Ultra}

\section{Culture reference standard}

We did not identify any studies that evaluated Xpert Ultra in peritoneal tissue against culture.

\section{Composite reference standard}

We did not identify any studies that evaluated Xpert Ultra in peritoneal tissue against a composite reference standard.

\section{Xpert MTB/RIF}

\section{Culture reference standard}

In peritoneal tissue, Xpert MTB/RIF sensitivity and specificity against culture were $50 \%$ (7 to 93 ) and 92\% (73 to 99) (1 study; 28 participants; Bera 2015); Appendix 10.

\section{Composite reference standard}

We did not identify any studies that evaluated Xpert MTB/RIF in peritoneal tissue against a composite reference standard.

\section{L: Xpert Ultra and Xpert MTB/RIF testing in pericardial fluid for pericardial tuberculosis}

\section{Xpert Ultra}

\section{Culture reference standard}

We did not identify any studies that evaluated Xpert Ultra in pericardial fluid against culture.

\section{Composite reference standard}

We did not identify any studies that evaluated Xpert Ultra in pericardial fluid against a composite reference standard.

\section{Xpert MTB/RIF}

\section{Culture reference standard}

Fourteen studies evaluated Xpert MTB/RIF in pericardial fluid against culture (Al-Ateah 2012; Blaich 2014; Causse 2011; Kim 2015a; Ozkutuk 2014; Pandie 2014; Peñata 2016; Safianowska 2012; Sharma 2014; Suzana 2016; Ullah 2017; Vadwai 2011; Zeka 2011; Zmak 2013). Xpert MTB/RIF sensitivity ranged from $0 \%$ to $100 \%$ and specificity ranged from $69 \%$ to $100 \%$ (Appendix 10). Nine studies (Al-Ateah 2012; Blaich 2014; Causse 2011; Kim 2015a; Ozkutuk 2014; Peñata 2016; Safianowska 2012; Vadwai 2011; Zmak 2013) did not contribute data to the meta-analysis because either sensitivity or specificity was not estimable.

In pericardial fluid, Xpert MTB/RIF pooled sensitivity and specificity against culture were $61.4 \%$ (32.4 to 82.4 ) and $89.7 \%$ (74.9 to 99.0 ), (5 studies; 181 participants, 57 (31.5\%) with tuberculosis); Table 2; Appendix 10.

\section{Composite reference standard}

We did not identify any studies that evaluated Xpert MTB/RIF in pericardial fluid against a composite reference standard.

\section{M: Xpert Ultra and Xpert MTB/RIF testing in blood for disseminated tuberculosis}

Xpert Ultra

\section{Culture reference standard}

We did not identify any studies that evaluated Xpert Ultra in blood against culture.

\section{Composite reference standard}

We did not identify any studies that evaluated Xpert Ultra in blood against a composite reference standard.

\section{Xpert MTB/RIF}

\section{Culture reference standard}

Two studies evaluated Xpert MTB/RIF in blood against culture (Feasey 2013; Zmak 2013). However, only one of these studies reported tuberculosis culture-positives. Xpert MTB/RIF sensitivity and specificity against culture were $56 \%$ (21 to 86 ) and $94 \%$ (85 to 98) (1 study; 74 participants, 9 (12.2\%) with tuberculosis (Feasey 2013)); Appendix 10.

\section{Composite reference standard}

We did not identify any studies that evaluated Xpert MTB/RIF in blood against a composite reference standard.

\section{Nontuberculous mycobacteria}

For Xpert Ultra, two studies provided data on a variety of NTMs that grew from the specimens tested to look for evidence of cross-reactivity. Donovan 2020 assessed Xpert Ultra specificity in CSF from more than 100 participants with nontuberculous meningitis and found zero positive Xpert Ultra results in those with a probable or possible diagnosis of tuberculous meningitis and in any participant with a confirmed diagnosis of nontuberculous meningitis. Perez-Risco 2018 assessed Xpert Ultra specificity using 20 culture-positive NTM specimens (covering a total of 18 species) and found that Xpert Ultra was negative for all specimens.

For Xpert MTB/RIF, we previously reported that in 10 studies involving 6975 specimens with 141 NTMs, Xpert MTB/RIF was negative in all specimens (Kohli 2018).

\section{Detection of rifampicin resistance}

\section{Xpert Ultra and Xpert MTB/RIF testing for rifampicin resistance} Xpert Ultra

Five studies evaluated Xpert Ultra for detection of rifampicin resistance. Xpert Ultra sensitivity estimates varied from $50 \%$ to $100 \%$; specificity varied from $93 \%$ to $100 \%$; Figure 17 . One study reported zero participants with rifampicin resistance and thus sensitivity was not estimable (Chin 2019). Four studies contributed data to the bivariate meta-analysis (Sun 2019; Wang 2019; Wang 2020; Wu 2019). Xpert Ultra pooled sensitivity and specificity were $100.0 \%$ (95.1 to 100.0 ) and $100.0 \%$ (99.0 to 100.0), (4 studies; 129 participants, 24 (18.6\%) with rifampicin resistance); Table 2. 
Figure 17. Forest plots of Xpert Ultra and Xpert MTB/RIF sensitivity and specificity for rifampicin resistance. The squares represent the sensitivity and specificity of one study, the black line its confidence interval. FN: falsenegative; FP: false-positive; TN: true-negative; TP: true-positive.

Rifampicin resistance, Xpert MTB/RIF

Study

Blaich 2014

Ablanedo-Terrazas 2014

Hillemann 2011

Iram 2015

Feasey 2013

Ghariani 2015

Pandie 2014

Ozkutuk 2014

Lusiba 2014

Malbruny 2011

Sharma 2016

Safianowska 2012

Zmak 2013

Zeka 2011

Biadglegne 2014

Dhasmana 2014

Bera 2015

Al-Ateah 2012

Hanif 2011

Friedrich 2011

Gu 2015

Nhu 2014

Meldau 2014

Wang 2020

Rufai 2015

Pe\&\#241; ata 2016

Rufai $2017 \mathrm{~b}$

Vadwai 2011

Nataraj 2016

Sharma 2014

Li 2017

Du 2015

Ligthelm 2011

Rifampicin resistance, xpert Ultra

$\begin{array}{rrrrr}\text { TP } & \text { FP } & \text { FN } & \text { TN } & \text { Sensitivity [95\% CI] } \\ 0 & 0 & 0 & 17 & \text { Not estimable } \\ 0 & 1 & 0 & 14 & \text { Not estimable } \\ 0 & 1 & 0 & 24 & \text { Not estimable } \\ 0 & 0 & 0 & 4 & \text { Not estimable } \\ 0 & 0 & 0 & 5 & \text { Not estimable } \\ 0 & 0 & 0 & 75 & \text { Not estimable } \\ 0 & 0 & 0 & 28 & \text { Not estimable } \\ 0 & 1 & 0 & 31 & \text { Not estimable } \\ 0 & 0 & 0 & 25 & \text { Not estimable } \\ 0 & 0 & 0 & 12 & \text { Not estimable } \\ 0 & 0 & 0 & 7 & \text { Not estimable } \\ 0 & 0 & 0 & 3 & \text { Not estimable } \\ 0 & 0 & 0 & 7 & \text { Not estimable } \\ 0 & 0 & 0 & 21 & \text { Not estimable } \\ 2 & 1 & 0 & 26 & 1.00[0.16,1.00] \\ 1 & 0 & 0 & 26 & 1.00[0.03,1.00] \\ 1 & 0 & 0 & 1 & 1.00[0.03,1.00] \\ 2 & 0 & 0 & 14 & 1.00[0.16,1.00] \\ 1 & 0 & 0 & 10 & 1.00[0.03,1.00] \\ 1 & 0 & 0 & 4 & 1.00[0.03,1.00] \\ 6 & 0 & 0 & 18 & 1.00[0.54,1.00] \\ 3 & 0 & 0 & 104 & 1.00[0.29,1.00] \\ 1 & 0 & 0 & 4 & 1.00[0.03,1.00] \\ 5 & 0 & 0 & 21 & 1.00[0.48,1.00] \\ 1 & 0 & 0 & 17 & 1.00[0.03,1.00] \\ 1 & 0 & 0 & 28 & 1.00[0.03,1.00] \\ 3 & 0 & 0 & 22 & 1.00[0.29,1.00] \\ 39 & 5 & 1 & 80 & 0.97[0.87,1.00] \\ 28 & 0 & 1 & 121 & 0.97[0.82,1.00] \\ 26 & 3 & 1 & 211 & 0.96[0.81,1.00] \\ 11 & 0 & 1 & 47 & 0.92[0.62,1.00] \\ 9 & 2 & 1 & 31 & 0.90[0.55,1.00] \\ 1 & 0 & 1 & 26 & 0.50[0.01,0.99] \\ & & & & \\ & \end{array}$

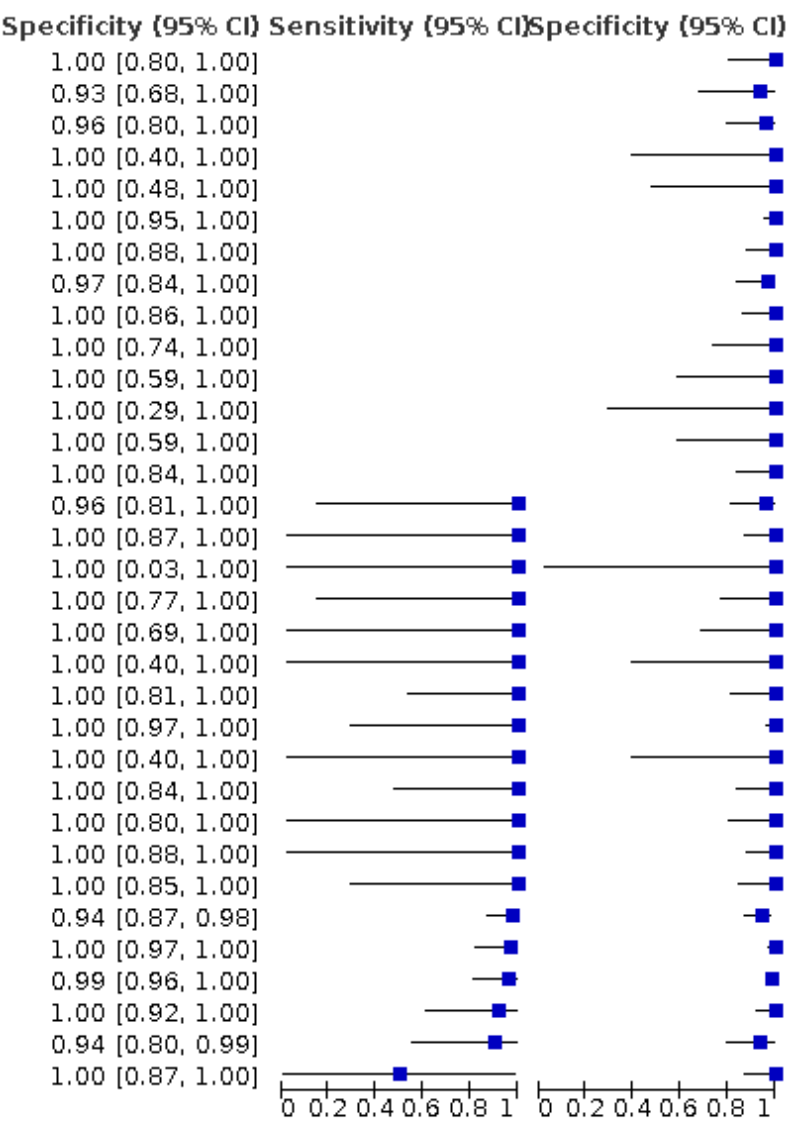

Sensitivity $(95 \%$ CI)Specificity $(95 \% \mathrm{CI})$

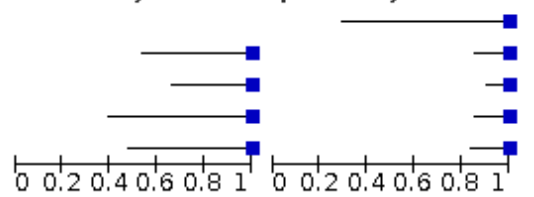

0.20 .40 .60 .8100 .20 .40 .60 .81

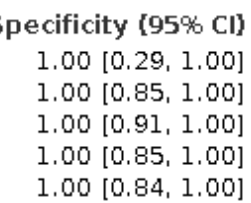

$\begin{aligned} \text { Not estimable } & 1.00[0.29,1.00] \\ 1.00[0.54,1.00] & 1.00[0.85,1.00] \\ 1.00[0.66,1.00] & 1.00[0.91,1.00] \\ 1.00[0.40,1.00] & 1.00[0.85,1.00] \\ 1.00[0.48,1.00] & 1.00[0.84,1.00]\end{aligned}$

\section{Xpert MTB/RIF}

Xpert MTB/RIF pooled sensitivity and specificity were $96.5 \%(91.9$ to 98.8 ) and $99.1 \%$ (98.0 to 99.7 ) (19 studies; 970 participants, 148 (15.3\%) with rifampicin resistance); Table 2; Figure 17.

\section{Indeterminate Xpert Ultra and Xpert MTB/RIF results for rifampicin} resistance

\section{Xpert Ultra}

Of the total 391 tests positive by Xpert Ultra, the proportion of indeterminate Xpert Ultra results for RIF resistance was 36.1\%. All of these indeterminate results were Xpert Ultra trace-positive.

\section{Xpert MTB/RIF}

We previously reported that for rifampicin resistance testing, of 1003 tests performed, the pooled proportion of indeterminate Xpert MTB/RIF results was $2.6 \%$ (95\% Crl 1.4 to 4.3 ) (Kohli 2018).

\section{DISCUSSION}

\section{Summary of main results}

This systematic review update summarizes the current literature and includes 69 unique studies on the accuracy of Xpert Ultra and Xpert MTB/RIF for extrapulmonary tuberculosis and rifampicin resistance. We identified 11 studies evaluating Xpert Ultra, an increase of 10 new studies since the original review (Kohli 2018). Unlike the original review, we limited inclusion to adults aged 15 
years and older. We also include a composite reference standard in addition to a culture reference standard, and have stratified all analyses by type of reference standard. Major findings from our review include the following.

- Xpert Ultra sensitivity for tuberculosis varied across different types of specimens (from $75.0 \%$ in pleural fluid to $89.4 \%$ in cerebrospinal fluid); Table 2

- Xpert MTB/RIF sensitivity for tuberculosis varied across different types of specimens (from $49.5 \%$ in pleural fluid to $97.9 \%$ in bone or joint aspirate); Table 2

- Xpert MTB/RIF specificity in cerebrospinal fluid, pleural fluid, urine, bone or joint aspirate, and peritoneal fluid was $\geq 96.9 \%$, against culture; overall, Xpert Ultra specificities were lower than those of Xpert MTB/RIF against culture, but against a composite reference standard results for both index tests were similar; Table 2

- In cerebrospinal fluid, Xpert Ultra sensitivity and specificity were $89.4 \%$ (79.1 to 95.6$)$ and $91.2 \%$ (83.2 to 95.7 ) against culture; Summary of findings 1 .

- In cerebrospinal fluid, Xpert MTB/RIF sensitivity and specificity were $71.1 \%$ (62.8 to 79.1 ) and $96.9 \%$ (95.4 to 98.0 ) against culture; Summary of findings 1

- In pleural fluid, Xpert Ultra sensitivity and specificity were $75.0 \%$ (58.0 to 86.4 ) and $87.0 \%$ (63.1 to 97.9 ) against culture; Summary of findings 2

- In pleural fluid, Xpert MTB/RIF sensitivity and specificity were $49.5 \%$ (39.8 to 59.9) and $98.9 \%$ (97.6 to 99.7) against culture; Summary of findings 2

- In lymph node aspirate, Xpert Ultra sensitivity and specificity were $70 \%$ (51 to 85 ) and 100\% (92 to 100) against a composite reference standard (1 study); Summary of findings 3

- In lymph node aspirate, Xpert MTB/RIF sensitivity and specificity were $81.6 \%$ (61.9 to 93.3 ) and $96.4 \%$ (91.3 to 98.6) against a composite reference standard; Summary of findings 3

- For rifampicin resistance, Xpert Ultra sensitivity and specificity were $100.0 \%$ (95.1 to 100.0 ) and $100.0 \%$ (99.0 to 100.0 ); Summary of findings 4

- For rifampicin resistance, Xpert MTB/RIF sensitivity and specificity were $96.5 \%$ (91.9 to 98.8 ) and $99.1 \%$ (98.0 to 99.7 ); Summary of findings 4

\section{Xpert Ultra and Xpert MTB/RIF testing in cerebrospinal fluid}

\section{(Summary of findings 1 )}

\section{Xpert Ultra}

Results of these studies indicate that in theory, for a population of 1000 people where 100 have tuberculosis meningitis on culture, 168 would be Xpert Ultra-positive: of these, 79 (47\%) would not have tuberculosis (false-positives); and 832 would be Xpert Ultra-negative: of these, 11 (1\%) would have tuberculosis (falsenegatives).

\section{Xpert MTB/RIF}

Results of these studies indicate that in theory, for a population of 1000 people where 100 have tuberculosis meningitis on culture, 99 would be Xpert MTB/RIF-positive: of these, 28 (28\%) would not have tuberculosis (false-positives); and 901 would be Xpert MTB/
RIF-negative: of these, 29 (3\%) would have tuberculosis (falsenegatives).

Rapid diagnosis of tuberculous meningitis is critical so that lifesaving treatment can be started promptly. Around 50\% of those affected die or experience disabling consequences (Thwaites 2013). Xpert Ultra was designed to improve tuberculosis detection, in particular in people with paucibacillary disease. The limit of detection for MTB is lower with Xpert Ultra (16 bacterial colony-forming units (cfu) per $\mathrm{mL}$ ) than with Xpert MTB/RIF (131 cfu per $\mathrm{mL}$ ) (Chakravorty 2017). In studies that compared Xpert Ultra and Xpert MTB/RIF in the same participants, we found Xpert Ultra to have higher pooled sensitivity $(89.0 \%)$ than Xpert MTB/RIF (62.2\%), and lower pooled specificity (91.0\%) than Xpert MTB/RIF (96.8\%) for tuberculous meningitis. In addition, in subgroup analyses we found slightly higher Xpert Ultra accuracy in studies that concentrated the cerebrospinal fluid (CSF): pooled sensitivity of $90.5 \%$ in concentrated specimens versus $88.4 \%$ in unconcentrated specimens; and pooled specificity of $91.9 \%$ in concentrated specimens versus $88.6 \%$ in unconcentrated specimens. We note that subgroup findings should be interpreted with caution, as there were only three studies and a small number of tuberculous meningitis cases included. The Tuberculous Meningitis International Research Consortium has recommended increasing the volume of CSF collected for diagnosis followed by centrifugation as a way of improving Xpert MTB/RIF assay sensitivity (Bahr 2016); however, we did not have sufficient data to investigate CSF collection volume. Increased Xpert MTB/RIF sensitivity in HIV-positive people compared with HIV-negative people has been reported, with the increased bacterial burden in tuberculosis and HIV co-infection proposed as the reason (Patel 2013). We had limited data to investigate this for Xpert Ultra as we identified only two studies in HIV-positive people, with sensitivities of 90\% (Bahr 2017) and 89\% (Cresswell 2020).

\section{Xpert Ultra and Xpert MTB/RIF testing in pleural fluid}

(Summary of findings 2)

\section{Xpert Ultra}

Results of these studies indicate that in theory, for a population of 1000 people where 100 have pleural tuberculosis on culture, 192 would be Xpert Ultra-positive: of these, 117 (61\%) would not have tuberculosis (false-positives); and 808 would be Xpert Ultra-negative: of these, 25 (3\%) would have tuberculosis (falsenegatives).

\section{Xpert MTB/RIF}

Results of these studies indicate that in theory, for a population of 1000 people where 100 have pleural tuberculosis on culture, 60 would be Xpert MTB/RIF-positive: of these, 10 (17\%) would not have tuberculosis (false-positives); and 940 would be Xpert MTB/ RIF-negative: of these, 50 (5\%) would have tuberculosis (falsenegatives).

With the bivariate model, we found Xpert Ultra to have higher pooled sensitivity (75.0\%) than Xpert MTB/RIF (49.6\%) and lower pooled specificity $(87.0 \%)$ than Xpert MTB/RIF (98.7\%) in pleural fluid against a culture reference standard, between-study comparison. Based on the latent class meta-analysis model, Xpert Ultra pooled sensitivity was comparable $(76.0 \%)$ and specificity higher $(99.5 \%)$ than what was obtained when culture was treated 
as having perfect accuracy. Xpert Ultra pooled sensitivity in pleural fluid was lower than that of CSF. One reason for the lower sensitivity of Xpert Ultra in pleural fluid could be the paucibacillary nature of pleural tuberculosis. Other possible reasons are contamination of blood or the presence of certain polymerase chain reaction (PCR) inhibitors in the pleural fluid (Pai 2004; Woods 2001). However, Theron and colleagues found that extrapulmonary specimens showed less evidence of PCR inhibition than pulmonary specimens, with bacterial load being more important for a positive Xpert MTB/ RIF result (Theron 2014b). Given that false-negative results were common (low sensitivity), a negative Xpert Ultra or Xpert MTB/RIF result may not be relied on to exclude tuberculosis.

\section{Xpert Ultra testing in lymph node aspirates}

\section{Xpert Ultra}

Results of these studies indicate that in theory, for a population of 1000 people where 100 have lymph node tuberculosis verified by a composite reference standard, 70 would be Xpert Ultra-positive: of these, $0(0 \%)$ would not have tuberculosis (false-positives); and 930 would be Xpert Ultra-negative: of these, 30 (3\%) would have tuberculosis (false-negatives).

\section{Xpert MTB/RIF}

Results of these studies indicate that in theory, for a population of 1000 people where 100 have lymph node tuberculosis verified by a composite reference standard, 118 would be Xpert MTB/RIFpositive: of these $37(31 \%)$ would not have tuberculosis (falsepositives); and 882 would be Xpert MTB/RIF-negative: of these 19 (2\%) would have tuberculosis (false-negatives).

Regarding Xpert testing for lymph node aspirate, it important to point out that although tissue biopsy provides material for histological examination which may be of substantial diagnostic value, a fluid specimen may be collected more easily. In addition, fine-needle aspiration of lymph nodes is well suited for use in resource-limited settings because the procedure is simple, easy to learn, minimally invasive, and inexpensive (Wright 2009b). Thus clinicians may want to consider fine-needle aspiration of lymph nodes before surgical biopsy.

In our review, using a standard bivariate meta-analysis model, Xpert MTB/RIF pooled specificity (defined by culture) in lymph node aspirate was $86.0 \%$, whereas with a composite reference standard pooled specificity increased to $95.9 \%$. Using a latent class meta-analysis model with informative priors, Xpert MTB/RIF pooled specificity increased to $99.5 \%$. In previous meta-analyses, Xpert MTB/RIF specificity for lymph node tuberculosis (aspirate and tissue) against culture as a reference standard was $94 \%$ (Denkinger 2014), 93\% (Maynard-Smith 2014), and 92\% (Penz 2015). See Table 8 . Using a composite reference standard (defined by the primary study authors), Denkinger 2014 found increased Xpert MTB/RIF specificity of $99 \%$ for lymph node tuberculosis (5 studies, 728 specimens). Thus, it appears that accuracy results depend in part on the choice of reference standard. Regarding the use of a composite reference standard, owing to differing definitions and difficulty in interpreting them, there is a risk of bias (Schiller 2016) (see section Strengths and weaknesses of the review).

We considered several reasons why the specificity of Xpert Ultra (78\%) and Xpert MTB/RIF (86.0\%) in lymph node aspirate against culture would be lower than in other extrapulmonary specimens.
Although not always reported, studies may have included participants receiving tuberculosis treatment. We previously reported that in a sensitivity analysis limiting inclusion to studies that involved participants not receiving tuberculosis treatment, specificity increased from $86 \%$ to $89 \%$ (Kohli 2018). We considered the type of culture used in the included studies because liquid culture is more sensitive than solid culture (American Thoracic Society 2000). Most studies used liquid culture or a combination of solid and liquid culture. The single study evaluating Xpert Ultra used liquid culture. Only two of the 15 studies (13\%) evaluating Xpert MTB/RIF exclusively used solid culture. Culture results may also be negative owing to inefficient specimen collection or errors in sampling, differing bacterial load, and contamination (Wright 2009b). Negative culture results in lymph node tuberculosis have previously been reported (Fontanilla 2011).

Another reason for negative culture results is that there may have been a decrease in live tuberculosis bacteria during processing with $\mathrm{N}$-acetyl-L-cysteine-sodium hydroxide, which is routinely used to homogenize, decontaminate, and liquefy non-sterile specimens, such as sputum, for mycobacterial culture (American Thoracic Society 2000). Harsh decontamination practices have been noted to contribute to false-negative culture results, especially in paucibacillary specimens (FIND 2017). Standards specify, "specimens collected from normally sterile sites may be placed directly into the culture medium" (American Thoracic Society 2000). CSF, pleural fluid, and lymph node aspirates are usually considered to be sterile specimens. It is our understanding that some laboratories do decontaminate sterile site specimens as a precaution against non-sterile collection procedures. In this review, $47 \%$ of the studies reported decontaminating lymph node aspirates before culture inoculation. We did not have sufficient data to further investigate laboratory practices.

In summary, several factors probably contributed to low Xpert MTB/ RIF specificity against culture in lymph node aspirate. The 'true' specificity of Xpert MTB/RIF in lymph node aspirate is likely to be higher owing to the aforementioned reasons. Xpert MTB/RIF specificity was higher against a composite reference standard and with application of latent class analysis, similar to that found in CSF, pleural fluid, and other specimens (Table 2; Table 3).

We investigated the prevalence of extrapulmonary tuberculosis (confirmed by culture) as a potential source of heterogeneity because changes in disease prevalence have often been found to be associated with other important changes, such as changes in the disease spectrum, which may affect diagnostic accuracy estimates (Leeflang 2013). For Xpert MTB/RIF, for pleural fluid and lymph node aspirate, we found that pooled sensitivity was higher in settings with higher tuberculosis prevalence. In all analyses, for both Xpert Ultra (CSF) and Xpert MTB/RIF (CSF, pleural fluid, and lymph node aspirate), specificity in settings with higher tuberculosis prevalence was similar or lower than in settings with lower tuberculosis prevalence. Findings from additional analyses are available in the previous version of this review (Kohli 2018).

\section{Xpert Ultra and Xpert MTB/RIF testing for rifampicin resistance}

(Summary of findings 4)

\section{Xpert Ultra}

Results of these studies indicate that in theory, for a population of 1000 people where 100 have rifampicin resistance, 100 would 
be Xpert Ultra-positive (resistant): of these, zero (0\%) would not have rifampicin resistance (false-positives); and 900 would be Xpert Ultra-negative (susceptible): of these, zero (0\%) would have rifampicin resistance.

\section{Xpert MTB/RIF}

Results of these studies indicate that in theory, for a population of 1000 people where 100 have rifampicin resistance, 105 would be Xpert MTB/RIF-positive (resistant): of these, 8 (8\%) would not have rifampicin resistance; and 895 would be Xpert MTB/RIF-negative (susceptible): of these, $3(0.3 \%)$ would have rifampicin resistance.

For detection of rifampicin resistance in extrapulmonary specimens, we found the sensitivity of Xpert Ultra (100\%) and Xpert MTB/RIF (96.7\%) and the specificity of Xpert Ultra (100\%) and Xpert MTB/RIF (99.1\%), to be comparable to estimates in pulmonary specimens: sensitivity (96\%) and specificity (98\%) (Horne 2019). We caution that the results for Xpert Ultra are based on only four studies, involving 129 participants, 24 (18.6\%) with rifampicin resistance. Nonetheless, these findings suggest that the use of Xpert Ultra and Xpert MTB/RIF in extrapulmonary specimens could assist in rapid diagnosis of rifampicin-resistant tuberculosis and early initiation of treatment for multidrug-resistant tuberculosis (MDR-TB).

Notably, concerns have been raised about rapid drug susceptibility testing (DST) methods, in particular automated mycobacteria growth indicator tube (MGIT) 960 for tuberculosis drug resistance using the recommended critical concentrations. As a priority, the WHO is planning to re-evaluate the critical concentrations for rifampicin (WHO 2018).

For Xpert Ultra, we found a high rate (36.1\%) of indeterminate rifampicin resistance results, all owing to trace call results. This finding was expected since, for trace call results, rifampicin resistance cannot be determined. Xpert Ultra incorporates two new multi-copy amplification targets (IS6110 and IS1081). Trace call indicates that only the multi-copy targets were detected, and not the tuberculosis-specific regions in the $r p o B$ gene. Resistance to rifampicin has mainly been associated mainly with mutations in a limited region of the $r p o B$ gene (Telenti 1993).

People-important outcomes, such as mortality, are especially relevant to patients, decision-makers, and the wider tuberculosis community. While performing this systematic review, we did not identify direct evidence of studies linking true-positives, falsepositives, true-negatives, and false-negatives to people-important outcomes when either Xpert Ultra or Xpert MTB/RIF was used to diagnose extrapulmonary tuberculosis. To our knowledge, for pulmonary tuberculosis, there have been two systematic reviews of randomized trials on the impact of the use of Xpert MTB/ RIF on health outcomes. Both reviews compared the effect of Xpert MTB/RIF and smear microscopy on all-cause mortality; Di Tanna and colleagues summarized the accuracy of Xpert MTB/ RIF in an individual patient-level data meta-analysis ( 3 trials, 8143 participants) (Di Tanna 2019) and Haraka and colleagues performed a random-effects meta-analysis, (5 trials, 10,409 participants (Haraka 2018; WHO Consolidated Guidelines (Module 3) 2020). In both reviews, Xpert MTB/RIF did not show a statistically significant effect on all-cause mortality, although the direction of effect was towards mortality reduction. Insufficient power to detect mortality in randomized trials measuring the impact of diagnostic tests on patient-important outcomes has been discussed previously as a limitation of such trials (Di Tanna 2019; Schumacher 2019). Larger sample sizes are needed to evaluate the effect of Xpert MTB/RIF on mortality, but achieving this is difficult in pragmatic situations. For example, Schumacher 2019 showed that a sample size of 31,000 participants would be needed if researchers were to plan a clusterrandomized diagnostic trial using the baseline mortality and effect size demonstrated by the individual patient data from Di Tanna 2019.

This review represents the most comprehensive review of the diagnostic accuracy of Xpert Ultra and Xpert MTB/RIF for extrapulmonary tuberculosis in adults. For Xpert MTB/RIF, our previous review (Kohli 2018) provides additional findings. These reviews provide evidence that may help countries to make decisions about scaling up the tests for programmatic management of tuberculosis and drug-resistant tuberculosis. Although the information in this review will help to inform such decisions, other factors such as resource requirements and feasibility (including stable electrical power supply, temperature control, and maintenance of the cartridge modules) will also be important considerations.

\section{Strengths and weaknesses of the review}

\section{Completeness of evidence}

This is a reasonably complete data set. We included any nonEnglish studies that we found from which we could obtain accuracy data. However, we acknowledge that we may have missed some studies despite the comprehensive search and our outreach to investigators. We included eight common forms of extrapulmonary tuberculosis in the review. However, for some of these forms, such as disseminated tuberculosis, data were insufficient to allow us to determine summary accuracy estimates. We did not include less common forms, such as cutaneous tuberculosis, ocular tuberculosis, female genital tuberculosis, and tuberculosis of the breast. We followed the Preferred Reporting Items for Systematic Reviews and Meta-Analyses of Diagnostic Test Accuracy (PRISMADTA) (Mclnnes 2018).

\section{Accuracy of the reference standards used}

In a systematic review of diagnostic test accuracy studies, the reference standard is the best available test to determine the presence or absence of the target condition. In this review, we used two reference standards: culture and a composite reference standard, both of which are known to be imperfect. While the composite reference standard is designed to have improved accuracy compared to culture alone, it may still lead to biased accuracy estimates of the index test, depending on various factors such as the accuracy of the different components; decision rules for combining them; prevalence of the target condition; and conditional dependence between the components and the index test (Schiller 2016). Conditional dependence between two imperfect tests arises when both tests make the same false-positive or false-negative errors more often than expected by chance (Naaktgeboren 2013). Hence, conditional dependence may arise between the index test and both reference standards we have used, as they are imperfect. As a consequence, we may overor underestimate the diagnostic accuracy of the index tests. An additional challenge with including a composite reference standard is that the definition of the composite reference standard may vary across studies, making it difficult to interpret the accuracy 
estimates. To adjust for the imperfect accuracy of culture, we applied a latent class model when evaluating Xpert MTB/RIF of studies. We added parameters for the sensitivity and specificity of culture and terms for conditional dependence to adjust for the dependence between Xpert MTB/RIF and culture among diseasepositive and disease-negative participants. In this way, we were able to improve estimation of both the pooled sensitivity and specificity of Xpert MTB/RIF, as well as between-study variability. An adequate number of studies is needed for a sufficiently robust model to estimate these additional parameters. We therefore found that we were unable to do the same for meta-analyses of the accuracy for Xpert Ultra owing to the small number of studies, many of which had small sample sizes resulting in zero cell counts.

Several factors may have contributed to false-negative culture results for the accuracy of the reference standard for lymph node aspirate in particular, including inefficient specimen collection and overly harsh decontamination. For this particular analysis, we were able to take advantage of the Bayesian estimation approach to incorporate prior information on Xpert MTB/RIF and culture specificity. This allowed us to make the best use of data from the included studies and our knowledge of the performance of Xpert MTB/RIF. We had insufficient data to apply the latent class model to data from the single study evaluating Xpert Ultra in lymph node aspirates.

Establishing a diagnosis of extrapulmonary tuberculosis would ideally include pursuing the diagnosis of pulmonary tuberculosis as well, because participants with tuberculosis may have both pulmonary and extrapulmonary tuberculosis and the lung may be the only site where the presence of tuberculosis can be established. Because of the difficulties involved in diagnosing HIVassociated tuberculosis, it is recommended that multiple cultures from sputum and other types of specimens be evaluated in people living with HIV (Bjerrum 2019; Shah 2016b). Given the limitations in the reference standard, we recommend that future studies consider using liquid culture because this is more sensitive than solid culture, and that researchers obtain multiple specimens for culture to confirm the diagnosis of extrapulmonary tuberculosis (Drain 2019).

Most studies included in this review used culture-based DST (either Löwenstein-Jensen (LJ) or mycobacteria growth indicator tube (MGIT) 960) as the reference standard for detection of rifampicin resistance. Concerns have been raised about rapid DST methods, using the recommended critical concentrations. The WHO is planning to prioritise a re-evaluation of the critical concentrations for rifampicin (WHO 2018).

We assessed the number of specimens with nontuberculous mycobacteria (NTMs) that were Xpert Ultra-positive. In two studies that reported 120 NTMs, Xpert Ultra was negative in all specimens. In the previous version of this review, among 10 studies that reported information comprising 141 NTMs, Xpert MTB/RIF was negative in all specimens (Kohli 2018).

\section{Quality and quality of reporting of the included studies}

Risk of bias was low for the participant selection, index test, and flow and timing domains and was high or unclear for the reference standard domain (most of these studies performed specimen sensitivity and specificity, for which there were a larger number in particular automated MGIT 960, for tuberculosis drug resistance

decontamination before culture inoculation). A limitation was that several studies included more than one specimen per participant, which artificially inflated the sample size of the study and may have led to overestimation or underestimation of the accuracy estimates. In general, studies were fairly well reported, although we corresponded with almost all primary study authors to ask for additional data and missing information. In several studies, accuracy data by site of extrapulmonary disease were not reported, and in a minority of studies, blinding was not reported. We strongly encourage the authors of future studies to follow the recommendations provided in the updated Standards for Reporting Diagnostic Accuracy (STARD) statement to improve the quality of reporting (Bossuyt 2015).

\section{Interpretability of subgroup analyses}

We investigated potential sources of heterogeneity in the different extrapulmonary specimens. Importantly, we found slightly higher Xpert Ultra accuracy in studies with concentrated cerebrospinal fluid (CSF) in comparison to unconcentrated specimens. We note that subgroup findings should be interpreted with caution, as there were only three studies and a small number of tuberculous meningitis cases included in these analyses.

\section{Comparison with other systematic reviews}

We are aware of several systematic reviews previously published on this topic that estimated summary accuracy of Xpert MTB/ RIF for distinct forms of extrapulmonary tuberculosis, as well as different forms of extrapulmonary tuberculosis combined (Table 8). We identified one systematic review that estimated summary accuracy of Xpert Ultra that found, for all forms of extrapulmonary tuberculosis combined, pooled sensitivity and specificity of $85.1 \%$ ( $95 \% \mathrm{Cl} 76.7$ to $90.8 \%$ ) and $95.7 \%$ (95\% $\mathrm{Cl} 87.9$ to $98.6 \%$ ), (7 studies; 1500 specimens) (Zhang 2020).

Compared with previous systematic reviews, our review extends the date of the search for potential studies for inclusion. Our strict inclusion criteria, e.g. excluding case-control studies, meant that some of the studies included in other reviews were excluded from ours.

\section{Applicability of findings to the review question}

For the participant selection domain, most studies had high or unclear concern for applicability because either participants were evaluated exclusively as inpatients in tertiary care or we were not sure about the clinical settings. We therefore cannot be sure about the applicability of our findings to primary care. Studies that take place in referral settings may include participants whose condition is more difficult to diagnose than are seen at lower levels of the health system. However, we recognize that classifying studies as primary, secondary, or tertiary care may not adequately account for differences in disease spectrum (Leeflang 2013). For the index and reference test domains, most studies had low concern for applicability.

\section{AUTHORS' CONCLUSIONS}

\section{Implications for practice}

In people presumed to have extrapulmonary tuberculosis, Xpert Ultra and Xpert MTB/RIF may be helpful in confirming the diagnosis. Sensitivity varies across different extrapulmonary specimens, while for most specimens specificity is high, the test rarely yielding a

Xpert MTB/RIF Ultra and Xpert MTB/RIF assays for extrapulmonary tuberculosis and rifampicin resistance in adults (Review) 49

Collaboration. 
positive result for people without tuberculosis. For tuberculous meningitis, Xpert Ultra had higher pooled sensitivity and lower pooled specificity than Xpert MTB/RIF against culture. Xpert Ultra and Xpert MTB/RIF had similar sensitivity and specificity for rifampicin resistance.

\section{Implications for research}

Future studies should perform comparisons of different tests, including Xpert Ultra, as this approach will reveal which tests (or strategies) yield superior diagnostic accuracy. For these studies, the preferred study design is one in which all participants receive all available diagnostic tests or are randomly assigned to receive one or another of the tests. Studies should include children and people living with HIV. Future research should acknowledge the concern associated with culture as a reference standard in paucibacillary specimens, and should consider ways to address this limitation.

Rapid point-of-care diagnostic tests for extrapulmonary tuberculosis are critically needed. Research groups should focus on developing diagnostic tests and strategies that use readilyavailable clinical specimens, such as urine, rather than specimens that require invasive procedures for collection.

\section{ACK N O WLEDGEMENTS}

The Academic Editors on this review were Dr Michael Eisenhut (Cochrane Infectious Diseases Group; CIDG) and Dr Mia SchmidtHansen (Cochrane Diagnostic Test Accuracy).

We are grateful to Vittoria Lutje, the CIDG Information Specialist, for help with the search strategy. The CIDG editorial base is funded by UK aid from the UK government for the benefit of low- and middle-income countries (project number 300342-104). The views expressed do not necessarily reflect the UK government's official policies.

We acknowledge Eleanor Ochodo, Centre for Evidence-based Health Care Department of Global Health, Stellenbosch, and Fredrick Haraka, Ifakara Health Institute, Bagamoyo, Tanzania, for the section on people-important outcomes. We thank Hannah Ryan, from the Tropical and Infectious Diseases Unit, Royal Liverpool University Hospital, for her help with the protocol. We thank Chunli Lu, from the Centre for Evidence-Based Chinese Medicine, Beijing University of Chinese Medicine, Beijing, and Marcela Perlwitz, Ivy Tech Community College of Indiana, West Lafayette, for help with translation. We thank all authors of the included studies for providing answers to our questions along with additional data. We thank the referees for their helpful comments. 


\section{R E F E R E N C E S}

\section{References to studies included in this review}

Ablanedo-Terrazas 2014 \{published data only\}

Ablanedo-Terrazas Y, Alvarado-de la Barrera C, HernandezJuan R, Ruiz-Cruz M, Reyes-Teran G. Xpert MTB/RIF for diagnosis of tuberculous cervical lymphadenitis in HIV-infected patients. Laryngoscope 2014;124(6):1382-5.

\section{Ajbani 2018 \{published data only\}}

Ajbani K, Kazi M, Naik S, Soman R, Shetty A, Rodrigues C. Utility of pyrosequencing for rapid detection of tubercular meningitis (TBM) and associated susceptibility directly from CSF specimens. Tuberculosis 2018;111:54-6.

\section{Al-Ateah 2012 \{published data only\}}

Al-Ateah SM, Al-Dowaidi MM, El-Khizzi NA. Evaluation of direct detection of Mycobacterium tuberculosis complex in respiratory and non-respiratory clinical specimens using the Cepheid Gene Xpert(R) system. Saudi Medical Journal 2012;33(10):1100-5.

\section{Antel 2020 \{published data only\}}

Antel K, Oosthuizen J, Malherbe F, Louw VJ, Nicol MP, Maartens G, et al. Diagnostic accuracy of the Xpert MTB/Rif Ultra for tuberculosis adenitis. BMC Infectious Diseases 2020;20(1):33.

\section{Azevedo 2018 \{published data only\}}

Azevedo RG, Dinallo FS, De Laurentis LS, Boulware DR, Vidal JE. Xpert MTB/RIF((R)) assay for the diagnosis of HIV-related tuberculous meningitis in Sao Paulo, Brazil. International Journal of Tuberculosis and Lung Disease 2018;22(6):706-7.

\section{Bahr 2015 \{published data only\}}

Bahr NC, Tugume L, Rajasingham R, Kiggundu R, Williams DA, Morawski B, et al. Improved diagnostic sensitivity for tuberculous meningitis with Xpert((R)) MTB/RIF of centrifuged CSF. International Journal of Tuberculosis and Lung Disease 2015;19(10):1209-15.

\section{Bahr 2017 \{published data only\}}

Bahr NC, Nuwagira E, Evans EE, Cresswell FV, Bystrom PV, Byamukama A, et al. Diagnostic accuracy of Xpert MTB/Rif Ultra for TB meningitis in HIV-infected adults: a prospective cohort study. Lancet Infectious Diseases 2017;18(1):68-75.

\section{Bera 2015 \{published data only\}}

Bera C, Michael JS, Burad D, Shirly SB, Gibikote S, Ramakrishna B, et al. Tissue Xpert TM MTB/Rif assay is of limited use in diagnosing peritoneal tuberculosis in patients with exudative ascites. Indian Journal of Gastroenterology 2015;34(5):395-8.

\section{Biadglegne 2014 \{published data only\}}

Biadglegne F, Mulu A, Rodloff A C, Sack U. Diagnostic performance of the Xpert MTB/RIF assay for tuberculous lymphadenitis on fine needle aspirates from Ethiopia. Tuberculosis (Edinburgh, Scotland) 2014;94(5):502-5.
Blaich 2014 \{published data only\}

Blaich A, Frei R. Performance of the Xpert MTB/RIF assay on nonrespiratory specimens and accuracy of this assay for detection of rifampin resistance in a low-prevalence setting. Journal of Clinical Microbiology 2014;52(2):706.

\section{Causse 2011 \{published data only\}}

Causse M, Ruiz P, Gutierrez-Aroca JB, Casal M. Comparison of two molecular methods for rapid diagnosis of extrapulmonary tuberculosis. Journal of Clinical Microbiology 2011;49(8):3065-7.

\section{Che 2017 \{published data only\}}

Che N, Yang X, Liu Z, Li K, Chen X. Rapid detection of cellfree Mycobacterium tuberculosis DNA in tuberculous pleural effusion. Journal of Clinical Microbiology 2017;55(5):1526-32.

\section{Chen 2019 \{published data only\}}

Chen C, Yang CG, Gao X, Lu ZZ, Tang FX, Cheng J, et al. Community-based active case finding for tuberculosis in rural western China: a cross-sectional study. International Journal of Tuberculosis and Lung Disease 2017;21(11):1134-9.

\section{Chin 2019 \{published data only\}}

Chin JH, Musubire AK, Morgan N, Pellinen J, Grossman S, Bhatt JM, et al. Xpert MTB/RIF Ultra for detection of Mycobacterium tuberculosis in cerebrospinal fluid. Journal of Clinical Microbiology 2019;57(6):pii: e00249-19.

Christopher 2013 \{published data only\} Christopher DJ, Schumacher SG, Michael JS, Luo R, Balamugesh T, Duraikannan P, et al. Performance of Xpert MTB/ RIF on pleural tissue for the diagnosis of pleural tuberculosis. European Respiratory Journal 2013;42(5):1427-9.

\section{Cresswell 2018 \{published data only\}}

Cresswell FV, Bangdiwala AS, Bahr NC, Trautner E, Nuwagira E, Ellis J, et al. Can improved diagnostics reduce mortality from tuberculous meningitis? Findings from a 6.5-year cohort in Uganda. Wellcome Open Research 2018;3:64.

\section{Cresswell 2020 \{published data only\}}

Cresswell FV, Tugume L, Bahr NC, Kwizera R, Bangdiwala AS, Musubire AK, et al. Xpert MTB/RIF Ultra for the diagnosis of HIVassociated tuberculous meningitis: a prospective validation study. Lancet Infectious Disease 2020;20(3):308-17.

Dhasmana 2014 \{published data only\}

Dhasmana DJ, Ross C, Bradley CJ, Connell DW, George PM, Singanayagam A, et al. Performance of Xpert MTB/RIF in the diagnosis of tuberculous mediastinal lymphadenopathy by endobronchial ultrasound. Annals of the American Thoracic Society 2014;11(3):392-6.

\section{Dhooria 2016 \{published data only\}}

Dhooria S, Gupta N, Bal A, Sehgal IS, Aggarwal A, Sethi S, et al. Role of Xpert MTB/RIF in differentiating tuberculosis from sarcoidosis in patients with mediastinal lymphadenopathy undergoing EBUS-TBNA: a study of 147 patients. Sarcoidosis Vasculitis and Diffuse Lung Disease 2016;33:258-266. 
Donovan 2020 \{published data only\}

Donovan J, Anh Thu DD, Phu NH, Dung VT, Quang TP, Nghia HDT, et al. Xpert MTB/RIF Ultra versus Xpert MTB/RIF for the diagnosis of tuberculous meningitis: a prospective, randomised, diagnostic accuracy study. Lancet Infectious Disease 2020;20:299-307.

\section{Du 2015 \{published data only\}}

Du J, Huang Z, Luo Q, Xiong G, Xu X, Li W, et al. Rapid diagnosis of pleural tuberculosis by Xpert MTB/RIF assay using pleural biopsy and pleural fluid specimens. Journal of Research In Medical Sciences 2015;20(1):26-31.

\section{El-Din 2019 \{published data only\}}

El-Din MG, Sobh E, Adawy Z, Farghaly N. Diagnostic utility of gene X-pert in the diagnosis of tuberculous pleural effusion. Infectious Diseases 2019;51(3):227-9.

\section{Feasey 2013 \{published data only\}}

Feasey NA, Banada PP, Howson W, Sloan DJ, Mdolo A, Boehme $\mathrm{C}$, et al. Evaluation of Xpert MTB/RIF for detection of tuberculosis from blood samples of HIV-infected adults confirms Mycobacterium tuberculosis bacteremia as an indicator of poor prognosis. Journal of Clinical Microbiology 2013;51(7):2311-6.

\section{Friedrich 2011 \{published data only\}}

Friedrich SO, Von Groote-Bidlingmaier F, Diacon AH. Xpert MTB/RIF assay for diagnosis of pleural tuberculosis. Journal of Clinical Microbiology 2011;49(12):4341-2.

\section{Ghariani 2015 \{published data only\}}

Ghariani A, Jaouadi T, Smaoui S, Mehiri E, Marouane C, Kammoun S, et al. Diagnosis of lymph node tuberculosis using the GeneXpert MTB/RIF in Tunisia. International Journal of Mycobacteriology 2015;4(4):270-5.

\section{Gu 2015 \{published data only\}}

Gu Y, Wang G, Dong W, Li Y, Ma Y, Shang Y, et al. Xpert MTB/RIF and GenoType MTBDRplus assays for the rapid diagnosis of bone and joint tuberculosis. International Journal of Infectious Diseases 2015;36:27-30.

\section{Hanif 2011 \{published data only\}}

Hanif SN, Eldeen HS, Ahmad S, Mokaddas E. GeneXpert(R) MTB/RIF for rapid detection of Mycobacterium tuberculosis in pulmonary and extra-pulmonary samples. International Journal of Tuberculosis and Lung Disease 2011;15(9):1274-5.

\section{Heemskerk 2018 \{published data only\}}

Heemskerk AD, Donovan J, Thu DD, Marais S, Chaidir L, Dung VT, et al. Improving the microbiological diagnosis of tuberculous meningitis: a prospective, international, multicentre comparison of conventional and modified ZiehlNeelsen stain, GeneXpert, and culture of cerebrospinal fluid. Journal of Infection 2018;77(6):509-15.

\section{Hillemann 2011 \{published data only\}}

Hillemann D, Rusch-Gerdes S, Boehme C, Richter E. Rapid molecular detection of extrapulmonary tuberculosis by the automated GeneXpert MTB/RIF system. Journal of Clinical Microbiology 2011;49(4):1202-5.

Iram 2015 \{published data only\}

Iram S, Zeenat A, Hussain S, Wasim Yusuf N, Aslam M. Rapid diagnosis of tuberculosis using Xpert MTB/RIF assay - report from a developing country. Pakistan Journal of Medical Sciences 2015;31(1):105-10.

Kim 2015a \{published data only\}

Kim YW, Kwak N, Seong MW, Kim EC, Yoo CG, Kim YW, et al. Accuracy of the Xpert(R) MTB/RIF assay for the diagnosis of extra-pulmonary tuberculosis in South Korea. International Journal of Tuberculosis and Lung Disease 2015;19(1):81-6.

\section{Li 2017 \{published data only\}}

Li Y, Pang Y, Zhang T, Xian X, Wang X, Yang J, et al. Rapid diagnosis of extrapulmonary tuberculosis with Xpert Mycobacterium tuberculosis/rifampicin assay. Journal of Medical Microbiology 2017;66(7):910-4.

\section{Liang 2019 \{published data only\}}

Liang Q, Pang Y, Yang Y, Li H, Guo C, Yang X, et al. An improved algorithm for rapid diagnosis of pleural tuberculosis from pleural effusion by combined testing with GeneXpert MTB/RIF and an anti-LAM antibody-based assay. BMC Infectious Diseases 2019;19(1):548.

\section{Ligthelm 2011 \{published data only\}}

Ligthelm LJ, Nicol MP, Hoek KG, Jacobson R, Van Helden PD, Marais BJ, et al. Xpert MTB/RIF for rapid diagnosis of tuberculous lymphadenitis from fine-needle-aspiration biopsy specimens. Journal of Clinical Microbiology 2011;49(11):3967-70.

\section{Lusiba 2014 \{published data only\}}

Lusiba JK, Nakiyingi L, Kirenga BJ, Kiragga A, Lukande R, Nsereko M, et al. Evaluation of Cepheid's Xpert MTB/Rif test on pleural fluid in the diagnosis of pleural tuberculosis in a high prevalence HIV/TB setting. PLOS One 2014;9(7):e102702. [DOI: 10.1371/journal.pone.0102702]

\section{Malbruny 2011 \{published data only\}}

Malbruny B, Le Marrec G, Courageux K, Leclercq R, Cattoir V. Rapid and efficient detection of Mycobacterium tuberculosis in respiratory and non-respiratory samples. International Journal of Tuberculosis and Lung Disease 2011;15(4):553-5.

\section{Meldau 2014 \{published data only\}}

Meldau R, Peter J, Theron G, Calligaro G, Allwood B, Symons G, et al. Comparison of same day diagnostic tools including Gene Xpert and unstimulated IFN-gamma for the evaluation of pleural tuberculosis: a prospective cohort study. BMC Pulmonary Medicine 2014;14:58. [DOI: 10.1186/1471-2466-14-58]

Meldau 2019 \{published data only\}

Meldau R, Randall P, Pooran A, Limberis J, Makambwa E, Dhansay M, et al. Same day tools, including Xpert Ultra and unstimulated IFN-gamma, for the rapid diagnosis of pleural tuberculosis - a prospective observational study. Journal of Clinical Microbiology 2019;57(9):e00614-19. 


\section{Metcalf 2018 \{published data only\}}

Metcalf T, Soria J, Montano SM, Ticona E, Evans CA, Huaroto L, et al. Evaluation of the GeneXpert MTB/RIF in patients with presumptive tuberculous meningitis. PLOS One 2018;13(6):e0198695.

\section{Nataraj 2016 \{published data only\}}

Nataraj G, Kanade S, Mehta P. Xpert((R)) MTB/RIF for improved case detection of extra-pulmonary TB in a tertiary care setting in urban India. International Journal of Tuberculosis and Lung Disease 2016;20(7):890-4.

Nhu 2014 \{published data only\}

Nhu NT, Heemskerk D, Thu do DA, Chau TT, Mai NT, Nghia HD, et al. Evaluation of GeneXpert MTB/RIF for diagnosis of tuberculous meningitis. Journal of Clinical Microbiology 2014;52(1):226-33.

\section{Ozkutuk 2014 \{published data only\}}

Ozkutuk N, Surucuoglu S. Evaluation of the Xpert MTB/RIF assay for the diagnosis of pulmonary and extrapulmonary tuberculosis in an intermediate-prevalence setting [Orta Prevalanslı Bölgede Akciğer ve Akciğer DışıTüberküloz Tanısında Xpert MTB/RIF Testinin Değerlendirilmesi]. Mikrobiyoloji Bulteni 2014;48(2):223-32.

\section{Pandie 2014 \{published data only\}}

Pandie S, Peter JG, Kerbelker ZS, Meldau R, Theron G, Govender U, et al. Diagnostic accuracy of quantitative PCR (Xpert MTB/RIF) for tuberculous pericarditis compared to adenosine deaminase and unstimulated interferon-gamma in a high burden setting: a prospective study. BMC Medicine 2014;12:101. [DOI: 10.1186/1741-7015-12-101]

\section{Patel 2013 \{published data only\}}

Patel VB, Theron G, Lenders L, Matinyena B, Connolly C, Singh R, et al. Diagnostic accuracy of quantitative PCR (Xpert MTB/ RIF) for tuberculous meningitis in a high burden setting: a prospective study. PLOS Medicine 2013;10(10):e1001536. [DOI: 10.1371/journal.pmed.1001536]

\section{Peñata 2016 \{published data only\}}

Peñata A, Salazar R, Castano T, Bustamante J, Ospina S. Molecular diagnosis of extrapulmonary tuberculosis and sensitivity to rifampicin with an automated real-time method [Diagnóstico molecular de tuberculosis extrapulmonary sensibilidad a rifampicina con un método automatizado en tiempo real]. Biomedica 2016;36(Suppl 1):78-89.

\section{Perez-Risco 2018 \{published data only\}}

Perez-Risco D, Rodriguez-Temporal D, Valledor-Sanchez I, Alcaide F. Evaluation of the Xpert MTB/RIF Ultra assay for direct detection of Mycobacterium tuberculosis complex in smear-negative extrapulmonary samples. Journal of Clinical Microbiology 2018;56(9):pii: e00659-18.

\section{Rakotoarivelo 2018 \{published data only\}}

Rakotoarivelo R, Ambrosioni J, Rasolofo V, Raberahona M, Rakotosamimanana N, Andrianasolo R, et al. Evaluation of the Xpert MTB/RIF assay for the diagnosis of smear-negative pulmonary and extrapulmonary tuberculosis in Madagascar. International Journal of Infectious Diseases 2018;69:20-5.

Rufai 2015 \{published data only\}

Rufai SB, Singh A, Kumar P, Singh J, Singh S. Performance of Xpert MTB/RIF Assay in diagnosis of pleural tuberculosis by use of pleural fluid samples. Journal of Clinical Microbiology 2015;53(11):3636-8.

Rufai 2017a \{published data only\}

Rufai SB, Singh S, Singh A, Kumar P, Singh J, Vishal A. Performance of Xpert MTB/RIF on ascitic fluid samples for detection of abdominal tuberculosis. Journal of Laboratory Physicians 2017;9(1):47-52.

Rufai 2017b \{published data only\}

Rufai SB, Singh A, Singh J, Kumar P, Sankar MM, Singh S. Diagnostic usefulness of Xpert MTB/RIF assay for detection of tuberculous meningitis using cerebrospinal fluid. Journal of Infection 2017;75(2):125-31.

\section{Safianowska 2012 \{published data only\}}

Safianowska A, Walkiewicz R, Nejman-Gryz P, GrubekJaworska $H$. Two selected commercially based nucleic acid amplification tests for the diagnosis of tuberculosis. Pneumonologia Alergologia Polska 2012;80(1):6-12.

\section{Sarfaraz 2018 \{published data only\}}

Sarfaraz S, Iftikhar S, Memon Y, Zahir N, Hereker FF, Salahuddin N. Histopathological and microbiological findings and diagnostic performance of GeneXpert in clinically suspected tuberculous lymphadenitis. International Journal of Infectious Diseases 2018;76:73-81.

\section{Scott 2014 \{published data only\}}

Scott LE, Beylis N, Nicol M, Nkuna G, Molapo S, Berrie L, et al. Diagnostic accuracy of Xpert MTB/RIF for extrapulmonary tuberculosis specimens: establishing a laboratory testing algorithm for South Africa. Journal of Clinical Microbiology 2014:52(6):1818-23.

\section{Sharma 2014 \{published data only\}}

Sharma SK, Kohli M, Chaubey J, Yadav RN, Sharma R, Singh BK, et al. Evaluation of Xpert MTB/RIF assay performance in diagnosing extrapulmonary tuberculosis among adults in a tertiary care centre in India. European Respiratory Journal 2014;44(4):1090-3.

\section{Sharma 2016 \{published data only\}}

Sharma JB, Kriplani A, Dharmendra S, Chaubey J, Kumar S, Sharma SK. Role of Gene Xpert in diagnosis of female genital tuberculosis: a preliminary report. European Journal of Obstetrics \& Gynecology and Reproductive Biology 2016;207:237-8.

\section{Sharma 2018 \{published data only\}}

Sharma K, Sharma M, Chaudhary L, Modi M, Goyal M, Sharma N, et al. Comparative evaluation of Xpert MTB/RIF assay with multiplex polymerase chain reaction for the diagnosis of tuberculous meningitis. Tuberculosis (Edinburgh, Scotland) 2018 Dec;113:38-42. 
Siddiqi 2019 \{published data only\}

Siddiqi OK, Birbeck GL, Ghebremichael M, Mubanga E, Love S, Buback C, et al. Prospective cohort study on performance of cerebrospinal fluid (CSF) Xpert MTB/RIF, CSF Lipoarabinomannan (LAM) Lateral Flow Assay (LFA), and urine LAM LFA for diagnosis of tuberculous meningitis in Zambia. Journal of Clinical Microbiology 2019;57(8):pii: e00652-19.

\section{Sun 2019 \{published data only\}}

Sun Q, Wang S, Dong W, Jiang G, Huo F, Ma Y, et al. Diagnostic value of Xpert MTB/RIF Ultra for osteoarticular tuberculosis. Journal of Infection 2019 August;79(2):153-8.

\section{Suzana 2016 \{published data only\}}

Suzana S, Ninan MM, Gowri M, Venkatesh K, Rupali P, Michael JS. Xpert MTB/Rif for the diagnosis of extrapulmonary tuberculosis - an experience from a tertiary care centre in South India. Tropical Medicine \& International Health 2016;21(3):385-92.

\section{Tadesse 2015 \{published data only\}}

Tadesse M, Abebe G, Abdissa K, Aragaw D, Abdella K, Bekele A, et al. GeneXpert MTB/RIF assay for the diagnosis of tuberculous lymphadenitis on concentrated fine needle aspirates in high tuberculosis burden settings. PLOS One 2015;10(9):e0137471. [DOI: 10.1371/journal.pone.0137471]

\section{Trajman 2014 \{published data only\}}

Trajman A, Da Silva Santos Kleiz de Oliveira EF, Bastos ML, Belo Neto E, Silva EM, Da Silva Lourenco MC, et al. Accuracy of polymerase chain reaction for the diagnosis of pleural tuberculosis. Respiratory Medicine 2014;108(6):918-23.

\section{Ullah 2017 \{published data only\}}

Ullah I, Javaid A, Masud H, Ali M, Basit A, Ahmad W, et al. Rapid detection of Mycobacterium tuberculosis and rifampicin resistance in extrapulmonary tuberculosis and sputum smearnegative pulmonary suspects using Xpert MTB/RIF. Journal of Medical Microbiology 2017;66(4):412-8.

\section{Vadwai 2011 \{published data only\}}

Vadwai V, Boehme C, Nabeta P, Shetty A, Alland D, Rodrigues C. Xpert MTB/RIF: a new pillar in diagnosis of extrapulmonary tuberculosis? Journal of Clinical Microbiology 2011;49(7):2540-5.

\section{Van Rie 2013 \{published data only\}}

Van Rie A, Page-Shipp L, Mellet K, Scott L, Mkhwnazi M, Jong E, et al. Diagnostic accuracy and effectiveness of the Xpert MTB/ RIF assay for the diagnosis of HIV-associated lymph node tuberculosis. European Journal of Clinical Microbiology \& Infectious Diseases 2013;32(11):1409-15.

\section{Wang 2019 \{published data only\}}

Wang G, Wang S, Jiang G, Yang X, Huang M, Huo F, et al. Xpert MTB/RIF Ultra improved the diagnosis of paucibacillary tuberculosis: a prospective cohort study. Journal of Infection 2019;78(4):311-6.
Wang 2020 \{published data only\}

Wang G, Wang S, Yang X, Sun Q, Jiang G, Huang M, et al. Accuracy of Xpert MTB/RIF Ultra for the diagnosis of pleural TB in a multicenter cohort study. Chest 2020;157(2):268-75.

\section{Wu 2019 \{published data only\}}

Wu X, Tan G, Gao R, Yao L, Bi D, Guo Y, et al. Assessment of the Xpert MTB/RIF Ultra assay on rapid diagnosis of extrapulmonary tuberculosis. International Journal of Infectious Diseases 2019;81:91-6

\section{Zeka 2011 \{published data only\}}

Zeka AN, Tasbakan S, Cavusoglu C. Evaluation of the GeneXpert MTB/RIF assay for rapid diagnosis of tuberculosis and detection of rifampin resistance in pulmonary and extrapulmonary specimens. Journal of Clinical Microbiology 2011;49(12):4138-41.

Zmak 2013 \{published data only\}

Zmak L, Jankovic M, Jankovic VK. Evaluation of Xpert MTB/RIF assay for rapid molecular diagnosis of tuberculosis in a twoyear period in Croatia. International Journal of Mycobacteriology 2013;2(3):179-82.

\section{References to studies excluded from this review}

\section{Abong 2019 \{published data only\}}

Abong J, Dalay V, Langley I, Tomeny E, Marcelo D, Mendoza V, et al. Use of GeneXpert and the role of an expert panel in improving clinical diagnosis of smear-negative tuberculosis cases. PLOS One 2019;14(12):e0227093.

\section{Adejumo 2018 \{published data only\}}

Adejumo Olusola Adedeji, Olusola-Faleye Bolanle, Adepoju Victor, Bowale Abimbola, Adesola Sunday, Falana Ayodeji, et al. Prevalence of rifampicin resistant tuberculosis and associated factors among presumptive tuberculosis patients in a secondary referral hospital in Lagos Nigeria. African Health Sciences 2018;18(3):472-8.

\section{Afsar 2018 \{published data only\}}

Afsar I, Gunes M, Er H, Sener AG. Comparison of culture, microscopic smear and molecular methods in diagnosis of tuberculosis. Revista Espanola de Quimioterapia 2018;31(5):435-8.

\section{Ahmad 2018 \{published data only\}}

Ahmad R, Changeez M, Khan JS, Qureshi U, Tariq M, Malik S, et al. Diagnostic accuracy of peritoneal fluid GeneXpert in the diagnosis of intestinal tuberculosis, keeping histopathology as the gold standard. Cureus 2018;10(10):e3451.

\section{Akhter 2019 \{published data only\}}

Akhter N, Sumalani KK, Chawla D, Ahmed Rizvi N. Comparison between the diagnostic accuracy of Xpert MTB/Rif assay and culture for pleural tuberculosis using tissue biopsy. ERJ Open Research 2019;5(3):00065-2019.

\section{Ali 2018 \{published data only\}}

Ali A, Munir MA, Sarwar MI, Khaliq S, Munir MK, Rehman S. Tuberculous pleural effusions: efficacy utilization and 
comparison of various diagnostic techniques. Pakistan Journal of Medical and Health Sciences 2018;12(4):1570-3.

\section{Allahyartorkaman 2019 \{published data only\}}

Allahyartorkaman M, Mirsaeidi M, Hamzehloo G, Amini S, Zakiloo M, Nasiri MJ. Low diagnostic accuracy of Xpert MTB/ RIF assay for extrapulmonary tuberculosis: a multicenter surveillance. Scientific Reports 2019;9(1):18515.

\section{Alvarez Uria 2012 \{published data only\}}

Alvarez-Uria G, Azcona JM, Midde M, Naik PK, Reddy S, Reddy R. Rapid diagnosis of pulmonary and extrapulmonary tuberculosis in HIV-infected patients. Comparison of LED fluorescent microscopy and the GeneXpert MTB/RIF assay in a district hospital in India. Tuberculosis Research and Treatment 2012;2012:932862. [DOI: 10.1155/2012/932862]

\section{Andrey 2015 \{published data only\}}

Andrey DO, Hinrikson H, Renzi G, Hibbs J, Adler D, Schrenzel J. Xpert((R)) MTB/RIF assay sensitivity with different methods of CSF processing for the diagnosis of TB meningitis. International Journal of Tuberculosis and Lung Disease 2015;19(12):1555-6.

\section{Armand 2011 \{published data only\}}

Armand S, Vanhuls P, Delcroix G, Courcol R, Lemaitre N. Comparison of the Xpert MTB/RIF test with an IS6110-TaqMan real-time PCR assay for direct detection of Mycobacterium tuberculosis in respiratory and nonrespiratory specimens. Journal of Clinical Microbiology 2011;49(5):1772-6.

\section{Arockiaraj 2015 \{published data only\}}

Arockiaraj J, Amritanand R, Krishnan V, Sundararaj G, Michael J. GeneXpert polymerase chain reaction (PCR) Test: role in the diagnosis of tubercular spondylodiscitis. Spine Journal 2015;15(10 Suppl 1):200S-201S.

\section{Arockiaraj 2017 \{published data only\}}

Arockiaraj J, Michael JS, Amritanand R, David KS, Krishnan V. The role of Xpert MTB/RIF assay in the diagnosis of tubercular spondylodiscitis. European Spine Journal 2017;26(12):3162-9.

\section{Arockiaraj 2019a \{published data only\}}

Arockiaraj J, Karthik R, Michael J S, Amritanand R, David KS, Krishnan V, et al. 'Need of the hour': early diagnosis and management of multidrug resistant tuberculosis of the spine: an analysis of 30 patients from a "high multidrug resistant tuberculosis burden" country. Asian Spine Journal 2019;13(2):265-71.

\section{Arockiaraj 2019b \{published data only\}}

Arockiaraj J, Robert M, Rose W, Amritanand R, David KS, Krishnan V. Early detection and analysis of children with multidrug-resistant tuberculosis of the spine. Asian Spine Journal 2019;13(1):77-85.

\section{Atherton 2018 \{published data only\}}

Atherton RR, Cresswell FV, Ellis J, Skipper C, Tadeo KK, Mugumya G, et al. Detection of Mycobacterium tuberculosis in urine by Xpert MTB/RIF Ultra: a useful adjunctive diagnostic tool in HIV-associated tuberculosis. International Journal of Infectious Diseases 2018;75:92-4.

\section{Aydemir 2019 \{published data only\}}

Aydemir O, Karakece E, Koroglu M, Altindis M, Terzi HA. Comparison of the GeneXpert MTB/RIF test and conventional methods in the diagnosis of Mycobacterium tuberculosis. Clinical Laboratory 2019;65(1):1-6.

Bablishvili 2015 \{published data only\}

Bablishvili N, Tukvadze N, Avaliani Z, Blumberg HM, Kempker RR. A comparison of the Xpert MTB/RIF and GenoTypeMTBDRplus assays in Georgia. International Journal of Tuberculosis and Lung Disease 2015;19(6):676-8.

\section{Bahr 2018a \{published data only\}}

Bahr NC, Halupnick R, Linder G, Kiggundu R, Nabeta HW, Williams DA, et al. Delta-like 1 protein, vitamin D binding protein and fetuin for detection of Mycobacterium tuberculosis meningitis. Biomarkers in Medicine 2018;12(7):707-16.

\section{Bahr 2018b \{published data only\}}

Bahr NC, Nuwagira E, Evans EE, Cresswell FV, Bystrom PV, Byamukama A, et al. Diagnostic accuracy of Xpert MTB/RIF Ultra for tuberculous meningitis in HIV-infected adults: a prospective cohort study. Lancet Infectious Diseases 2018;18(1):68-75.

\section{Bahr 2019 \{published data only\}}

Bahr NC, Meintjes G, Boulware DR. Inadequate diagnostics: the case to move beyond the bacilli for detection of meningitis due to Mycobacterium tuberculosis. Journal of Medical Microbiology 2019;68(5):755-60.

\section{Baikunje 2019 \{published data only\}}

Baikunje N, Behera D, Rajwanshi A, Sharma M, Sharma A, Sharma K. Comparative evaluation of loop-mediated isothermal amplification (LAMP) assay, GeneXpert MTB/Rif and multiplex PCR for the diagnosis of tubercular lymphadenitis in HIV-infected patients of North India. Molecular and Cellular Probes 2019;48:101459.

\section{Bajrami 2016 \{published data only\}}

Bajrami R, Mulliqi G, Kurti A, Lila G, Raka L. Comparison of GeneXpert MTB/RIF and conventional methods for the diagnosis of tuberculosis in Kosovo. Journal of Infection in Developing Countries 2016;10(4):418-22.

\section{Balcha 2014 \{published data only\}}

Balcha TT, Sturegard E, Winqvist N, Skogmar S, Reepalu A, Jemal ZH, et al. Intensified tuberculosis case-finding in HIVpositive adults managed at Ethiopian health centers: diagnostic yield of Xpert MTB/RIF compared with smear microscopy and liquid culture. PLOS One 2014;9(1):e85478. [DOI: 10.1371/ journal.pone.0085478]

Bankar 2018 \{published data only\}

Bankar S, Set R, Sharma D, Shah D, Shastri J. Diagnostic accuracy of Xpert MTB/RIF assay in extrapulmonary tuberculosis. Indian Journal of Medical Microbiology 2018;36(3):357-63.

\section{Bemba 2017 \{published data only\}}

Bemba EL, Moukassa D, Ouedraogo AR, Okombi FH, Bopaka RG, Koumeka PP, et al. Performance of GeneXpert MTB / RIF in the 
diagnosis of pleural tuberculosis in Brazzaville: a preliminary report [Performance du GeneXpert MTB/RIF dans le diagnostic de la tuberculose pleurale à Brazzaville: étude préliminaire]. Health Sciences and Diseases 2017;18(3):21-7.

Ben Saad 2018 \{published data only\}

Ben Saad S, Kallel N, Gharsalli H, Kwas H, El Gharbi L, Ghedira H, et al. Cold abscess in the immunocompetent subject. Tunisie Medicale 2018;96(5):302-6.

\section{Bhardwaj 2019 \{published data only\}}

Bhardwaj A, Khan S, Kumar A, George L, Mehta A, Radhakrishnan K. Assessing the utility of GeneXpert MTB/ Rif assay in a tertiary care centre in Southern India with established microscopy and liquid culture facilities. Journal of the Association of Physicians of India 2019;67(8):31-4.

Bhatia 2016 \{published data only\}

Bhatia R, Dayal R, Jindal S, Agarwal D, Goyal A. GeneXpert for diagnosis of tubercular meningitis. Indian Journal of Pediatrics 2016;83(11):1353-5.

\section{Bholla 2016 \{published data only\}}

Bholla M, Kapalata N, Masika E, Chande H, Jugheli L, Sasamalo M, et al. Evaluation of Xpert(R) MTB/RIF and Ustar EasyNAT TB IAD for diagnosis of tuberculous lymphadenitis of children in Tanzania: a prospective descriptive study. BMC Infectious Diseases 2016;16:246. [DOI: 10.1186/s12879-016-1578z]

\section{Biadglegne 2013 \{published data only\}}

Biadglegne F, Tesfaye W, Sack U, Rodloff AC. Tuberculous lymphadenitis in Northern Ethiopia: in a public health and microbiological perspectives. PLOS One 2013;8(12):e81918. [DOI: 10.1371/journal.pone.0081918]

\section{Bilgin 2016 \{published data only\}}

Bilgin K, Yanik K, Karadag A, Odabasi H, Tas H, Gunaydin M. Comparison of a real-time polymerase chain reaction-based system and Erlich-Ziehl-Neelsen method with culture in the identification of Mycobacterium tuberculosis. Turkish Journal of Medical Sciences 2016;46(1):203-6.

\section{Borraz-Noriega 2018 \{published data only\}}

Borraz-Noriega D, Robledo-Pascual JC, Torres-Perez JA, FloresBarrientos OI. Utility of nucleic acids detection GeneXpert tuberculosis (MTB/RIF) in respiratory and non-respiratory hospital reference samples. Medicina Interna de Mexico 2018;34(3):381-7.

\section{Boyles 2018 \{published data only\}}

Boyles T. Xpert Ultra's place in the diagnosis of tuberculous meningitis. Lancet Infectious Diseases 2018;18(3):248-9.

\section{Bunsow 2014 \{published data only\}}

Bunsow E, Ruiz-Serrano MJ, Lopez Roa P, Kestler M, Viedma DG, Bouza E. Evaluation of GeneXpert MTB/RIF for the detection of Mycobacterium tuberculosis and resistance to rifampin in clinical specimens. Journal of Infection 2014;68(4):338-43.

\section{Celik 2015 \{published data only\}}

Celik C, Gozel MG, Bakici MZ, Berk S, Ozsahin SL, Gulturk E. Applicability of Xpert MTB/RIF assay for routine diagnosis of tuberculosis: a four-year single-center experience. Turkish Journal of Medical Sciences 2015;45(6):1329-34.

\section{Chaidir 2018 \{published data only\}}

Chaidir L, Annisa J, Dian S, Parwati I, Alisjahbana A, Purnama F, et al. Microbiological diagnosis of adult tuberculous meningitis in a ten-year cohort in Indonesia. Diagnostic Microbiology and Infectious Disease 2018;91(1):42-6.

Chakraborty 2019 \{published data only\}

Chakraborty A, Ramaswamy S, Shivananjiah A J, Puttaswamy RB, Chikkavenkatappa N. The role of genexpert in the diagnosis of tubercular pleural effusion in India. Advances in Respiratory Medicine 2019;87(5):276-80.

\section{Chen 2016 \{published data only\}}

Chen ZF, Lao HL, Li XH, Wang J, Chen Q, Wang ZX, et al. Experimental study of GeneXpert((R)) system in the diagnosis of extra-pulmonary tuberculosis. Chinese Journal of Tuberculosis and Respiratory Diseases 2016;39(7):529-33.

\section{Chhajed 2019 \{published data only\}}

Chhajed PN, Vaidya PJ, Mandovra NP, Chavhan VB, Lele TT, Nair R, et al. EBUS-TBNA in the rapid microbiological diagnosis of drug-resistant mediastinal tuberculous lymphadenopathy. ERJ Open Research 2019;5(4):00008-2019.

Christopher 2018 \{published data only\}

Christopher DJ, Dinakaran S, Gupta R, James P, Isaac B, Thangakunam B. Thoracoscopic pleural biopsy improves yield of Xpert MTB/RIF for diagnosis of pleural tuberculosis. Respirology 2018;23(7):714-7.

\section{Coetzee 2014 \{published data only\}}

Coetzee L, Nicol MP, Jacobson R, Schubert PT, Van Helden PD, Warren RM, et al. Rapid diagnosis of pediatric mycobacterial lymphadenitis using fine needle aspiration biopsy. Pediatric Infectious Disease Journal 2014;33(9):893-6.

\section{Coleman 2015 \{published data only\}}

Coleman M, Finney LJ, Komrower D, Chitani A, Bates J, Chipungu GA, et al. Markers to differentiate between Kaposi's sarcoma and tuberculous pleural effusions in HIV-positive patients. International Journal of Tuberculosis and Lung Disease 2015;19(2):144-50.

\section{Creswell 2019 \{published data only\}}

Creswell J, Qin ZZ, Gurung R, Lamichhane B, Yadav DK, Prasai MK, et al. The performance and yield of tuberculosis testing algorithms using microscopy, chest $x$-ray, and Xpert MTB/RIF. Journal of Clinical Tuberculosis and Other Mycobacterial Diseases 2019;14:1-6.

Dahale 2019 \{published data only\}

Dahale AS, Puri AS, Kumar A, Dalal A, Agarwal A, Sachdeva S. Tissue Xpert (R) MTB/RIF assay in peritoneal tuberculosis: to be (done) or not to be (done). Cureus 2019;11(6):e5009. 
Das 2019 \{published data only\}

Das A, Anupurba S, Mishra OP, Banerjee T, Tripathi R. Evaluation of Xpert MTB/RIF assay for diagnosis of tuberculosis in children. Journal of Tropical Pediatrics 2019;65(1):14-20.

\section{Deggim 2013 \{published data only\}}

Deggim V, Somoskovi A, Voit A, Bottger EC, Bloemberg GV. Integrating the Xpert MTB/RIF assay into a diagnostic workflow for rapid detection of Mycobacterium tuberculosis in a low-prevalence area. Journal of Clinical Microbiology 2013;51(7):2396-9.

\section{Dharan 2016 \{published data only\}}

Dharan NJ, Blakemore R, Sloutsky A, Kaur D, Alexander RC, Ghajar M, et al. Performance of the G4 Xpert MTB/RIF assay for the detection of Mycobacterium tuberculosis and rifampin resistance: a retrospective case-control study of analytical and clinical samples from high- and low-tuberculosis prevalence settings. BMC Infectious Diseases 2016;16:764. [DOI: 10.1186/ s12879-016-2039-4]

\section{Diallo 2016 \{published data only\}}

Diallo AB, Kollo Al, Camara M, Lo S, Ossoga GW, Mbow M, et al. Performance of GeneXpert MTB / RIF in the diagnosis of extrapulmonary tuberculosis in Dakar: 2010-2015 [Performance du GeneXpert MTB/RIF ${ }^{\circledR}$ dans le diagnostic de la tuberculose extra-pulmonaire à Dakar: 2010-2015]. Pan African Medical Journal 2016;25:129. [DOI: 10.11604/pamj.2016.25.129.10065]

\section{Diop 2016 \{published data only\}}

Diop SA, Massaly A, Ka D, Manga NM, Fortes-Deguenonvo L, Ndour CT, et al. Use of GeneXpert test for the diagnosis of tuberculosis in the department of infectious diseases of FANN University Hospital [Utilisation du test GeneXpert pour le diagnostic de la tuberculose au service desmaladies infectieuses du CHNU de Fann]. Pan African Medical Journal 2016;23:244. [DOI: 10.11604/pamj.2016.23.244.7442]

\section{Edwards 2016 \{published data only\}}

Edwards S, Glynn P, David MD, Kamesh L. Diagnosing tuberculous peritonitis early in patients on peritoneal dialysis: use of Xpert MTB/RIF assay. Peritoneal Dialysis International 2016;36(4):461.

\section{Ejeta 2018 \{published data only\}}

Ejeta E, Beyene G, Bonsa Z, Abebe G. Xpert MTB/RIF assay for the diagnosis of Mycobacterium tuberculosis and rifampicin resistance in high Human Immunodeficiency Virus setting in Gambella regional state, Southwest Ethiopia. Journal of Clinical Tuberculosis and Other Mycobacterial Diseases 2018;12:14-20.

\section{Erdem 2014 \{published data only\}}

Erdem H, Ozturk-Engin D, Elaldi N, Gulsun S, Sengoz G, Crisan A, et al. The microbiological diagnosis of tuberculous meningitis: results of Haydarpasa-1 study. Clinical Microbiology and Infection 2014;20(10):0600-8. [DOI: 10.1111/1469-0691.12478]

\section{Fanosie 2016 \{published data only\}}

Fanosie A, Gelaw B, Tessema B, Tesfay W, Admasu A, Yitayew G. Mycobacterium tuberculosis complex and HIV co-infection among extrapulmonary tuberculosis suspected cases at the
University of Gondar Hospital, Northwestern Ethiopia. PLOS One 2016;11(3):e0150646. [DOI: 10.1371/journal.pone.0150646]

Fantahun 2019 \{published data only\}

Fantahun M, Kebede A, Yenew B, Gemechu T, Mamuye Y, Tadesse M, et al. Diagnostic accuracy of Xpert MTB/RIF assay and non-molecular methods for the diagnosis of tuberculosis lymphadenitis. PLOS One 2019;14(9):e0222402.

\section{Floridia 2017 \{published data only\}}

Floridia M, Ciccacci F, Andreotti M, Hassane A, Sidumo Z, Magid NA, et al. Tuberculosis case finding with combined rapid point-of-care assays (Xpert MTB/RIF and Determine TB LAM) in HIV-positive individuals starting antiretroviral therapy in Mozambique. Clinical Infectious Diseases 2017;65(11):1878-83.

\section{García 2017 \{published data only\}}

Garcia P, Balcells ME, Castillo C, Miranda C, Geoffroy E, Román JC, et al. Evaluation of Xpert ${ }^{\circledR}$ MTB/RIF technique for Mycobacterium tuberculosis complex detection in extrarespiratory specimens [Evaluation of Xpert(R) MTB/RIF technique for Mycobacterium tuberculosis complex detection in extra-respiratory specimens]. Revista Chilena de Infectologia 2017;34(4):333-9.

\section{García Cañete 2017 \{published data only\}}

García Cañete P, Balcells ME, Castillo C, Miranda C, Geoffroy E, Román JC, et al. Evaluation of Xpert ${ }^{\circledR}$ MTB/RIF technique for Mycobacterium tuberculosis complex detection in extrarespiratory specimens. Revista Chilena de Infectologia 2017;34(4):333-9.

\section{Gascoyne-Binzi 2012 \{published data only\}}

Gascoyne-Binzi DM, Robinson GA, English A, Collyns TA. Comparison of on-demand PCR testing for Mycobacterium tuberculosis with culture and batched PCR. Clinical Microbiology and Infection 2012;18:543. [DOI: doi.org/10.1111/ j.1469-0691.2012.03802.x]

\section{Gati 2018 \{published data only\}}

Gati S, Chetty R, Wilson D, Achkar J M. Utilization and clinical value of diagnostic modalities for tuberculosis in a high HIV prevalence setting. American Journal of Tropical Medicine and Hygiene 2018;99(2):317-22.

\section{Gautam 2018 \{published data only\}}

Gautam H, Agrawal SK, Verma SK, Singh UB. Cervical tuberculous lymphadenitis: clinical profile and diagnostic modalities. International Journal of Mycobacteriology 2018;7(3):212-6.

\section{Gautam 2019 \{published data only\}}

Gautam H, Singla M, Jain R, Lodha R, Kabra SK, Singh UB. Point-of-care urine lipoarabinomannan antigen detection for diagnosis of tuberculosis in children. International Journal of Tuberculosis and Lung Disease 2019;23(6):714-9.

\section{Gounden 2018 \{published data only\}}

Gounden S, Perumal R, Magula NP. Extrapulmonary tuberculosis in the setting of HIV hyperendemicity at a tertiary hospital in 
Durban, South Africa. Southern African Journal of Infectious Diseases 2018;33(3):57-64.

\section{Gulla 2019 \{published data only\}}

Gulla KM, Gunathilaka G, Jat KR, Sankar J, Karan M, Lodha R, et al. Utility and safety of endobronchial ultrasound-guided transbronchial needle aspiration and endoscopic ultrasound with an echobronchoscope-guided fine needle aspiration in children with mediastinal pathology. Pediatric Pulmonology 2019;54(6):881-5.

\section{Gursoy 2016 \{published data only\}}

Gursoy NC, Yakupogullari Y, Tekerekoglu MS, Otlu B. Evaluation of the diagnostic performance of Xpert MTB/RIF test for the detection of Mycobacterium tuberculosis and rifampin resistance in clinical samples [Klinik örneklerden Mycobacterium tuberculosis saptanması ve rifampin direnci tespitinde Xpert MTB/RIF testinin tanısal performansının değerlendirilmesi]. Mikrobiyoloji Bulteni 2016;50(2):196-204.

\section{Habeenzu 2017 \{published data only\}}

Habeenzu C, Nakajima C, Solo E, Bwalya P, Kajino K, Miller M, et al. Evaluation of in-house loop-mediated isothermal amplification for tuberculosis diagnosis compared with Xpert MTB/RIF. Journal of Infection in Developing Countries 2017;11(6):440-4.

\section{Habous 2019 \{published data only\}}

Habous M, Elimam MA, Kumar R, Deesi ZA. Evaluation of GeneXpert Mycobacterium tuberculosis/rifampin for the detection of Mycobacterium tuberculosis complex and rifampicin resistance in nonrespiratory clinical specimens. International Journal of Mycobacteriology 2019;8(2):132-7.

\section{Hanifa 2017 \{published data only\}}

Hanifa Y, Fielding KL, Chihota VN, Adonis L, Charalambous S, Foster $\mathrm{N}$, et al. A clinical scoring system to prioritise investigation for tuberculosis among adults attending HIV clinics in South Africa. PLOS One 2017;12(8):e0181519.

\section{Held 2014 \{published data only\}}

Held M, Laubscher M, Zar HJ, Dunn RN. GeneXpert polymerase chain reaction for spinal tuberculosis: an accurate and rapid diagnostic test. Bone \& Joint Journal 2014;96-b(10):1366-9.

\section{Held 2016 \{published data only\}}

Held M, Laubscher M, Mears S, Dix-Peek S, Workman L, Zar H, et al. Diagnostic accuracy of the Xpert MTB/RIF assay for extrapulmonary tuberculosis in children with musculoskeletal infections. Pediatric Infectious Disease Journal 2016;35(11):1165-8.

\section{Held 2017 \{published data only\}}

Held M, Laubscher M, Workman L, Zar HJ, Dunn R. Diagnostic accuracy of GeneXpert MTB/RIF in musculoskeletal tuberculosis: high sensitivity in tissue samples of HIV-infected and HIV-uninfected patients. South African Medical Journal 2017;107(10):854-8.

\section{Ioannidis 2010 \{published data only\}}

Ioannidis P, Papaventsis D, Nikolaou S, Karabela S, Konstantinidou E, Marinou I, et al. Tuberculosis resistance detection rate to the two main anti-TB drugs, isoniazid and rifampicin, using molecular techniques: experience of the Hellenic National Reference Center for Mycobacteria. Acta Microbiologica Hellenica 2010;55(2):175-82.

\section{loannidis 2011 \{published data only\}}

Ioannidis P, Papaventsis D, Karabela S, Nikolaou S, Panagi M, Raftopoulou E, et al. Cepheid GeneXpert MTB/RIF assay for Mycobacterium tuberculosis detection and rifampin resistance identification in patients with substantial clinical indications of tuberculosis and smear-negative microscopy results. Journal of Clinical Microbiology 2011;49(8):3068-70.

\section{Jain 2017 \{published data only\}}

Jain A, Singh PK, Singh U, Kumar V. Initial screening of extrapulmonary tuberculosis using the Xpert MTB/RIF assay improves case detection rates. International Journal of Tuberculosis and Lung Disease 2017;21(4):478-80.

\section{Jing 2017 \{published data only\}}

Jing H, Lu ZM, Deng YF, Gao DC, Li L, Graviss EA, et al. Evaluation of Xpert MTB/RIF in detection of pulmonary and extrapulmonary tuberculosis cases in China. International Journal of Clinical and Experimental Pathology 2017;10(4):4847-51.

\section{Jipa 2017 \{published data only\}}

Jipa R, Olaru I, Manea E, Merisor S, Hristea A. Rapid clinical score for the diagnosis of tuberculous meningitis: a retrospective cohort study. Annals of Indian Academy of Neurology 2017;20(4):363-6.

\section{Jorstad 2018 \{published data only\}}

Jorstad MD, Marijani M, Dyrhol-Riise AM, Sviland L, Mustafa T. MPT64 antigen detection test improves routine diagnosis of extrapulmonary tuberculosis in a low-resource setting: a study from the tertiary care hospital in Zanzibar. PLOS One 2018;13(5):e0196723.

\section{Joythi 2019 \{published data only\}}

Joythi A, Ratageri V, Illalu S, Fattepur SR, Wari PK. The utility of CSF Xpert MTB/RIF in diagnosis of tubercular meningitis in children. Indian Journal of Pediatrics 2019;86(12):1089-93.

\section{Kanade 2018 \{published data only\}}

Kanade S, Nataraj G, Mehta P, Shah D. Pattern of missing probes in rifampicin resistant TB by Xpert MTB/RIF assay at a tertiary care centre in Mumbai. Indian Journal of Tuberculosis 2019;66(1):139-43.

\section{Kashyap 2019 \{published data only\}}

Kashyap B, Goyal N, Hyanki P, Singh NP, Khanna A. Cartridgebased nucleic acid amplification test: a novel rapid diagnostic tool to study the burden of tuberculosis from a tertiary care hospital. Tropical Doctor 2019;49(4):274-81. 


\section{Kendall 2019 \{published data only\}}

Kendall EA, Kamoga C, Kitonsa PJ, Nalutaaya A, Salvatore PP, Robsky $\mathrm{K}$, et al. Empiric treatment of pulmonary TB in the Xpert era: correspondence of sputum culture, Xpert MTB/RIF, and clinical diagnoses. PLOS One 2019;14(7):e0220251.

\section{Kerkhoff 2017 \{published data only\}}

Kerkhoff AD, Barr DA, Schutz C, Burton R, Nicol MP, Lawn SD, et al. Disseminated tuberculosis among hospitalised HIV patients in South Africa: a common condition that can be rapidly diagnosed using urine-based assays. Scientific Reports 2017;7(1):10931.

\section{Khadka 2019 \{published data only\}}

Khadka P, Thapaliya J, Basnet RB, Ghimire GR, Amatya J, Rijal BP. Diagnosis of tuberculosis from smear-negative presumptive TB cases using Xpert MTB/Rif assay: a crosssectional study from Nepal. BMC Infectious Diseases 2019;19(1):1090.

\section{Khan 2018 \{published data only\}}

Khan AS, Ali S, Khan MT, Ahmed S, Khattak Y, Abduljabbar, et al. Comparison of GeneXpert MTB/RIF assay and LED-FM microscopy for the diagnosis of extrapulmonary tuberculosis in Khyber Pakhtunkhwa, Pakistan. Brazilian Journal of Microbiology 2018;49(4):909-13.

\section{Kilfoil 2015 \{published data only\}}

Kilfoil KM, Mayne E, Scott L, Stevens W. A high burden human immunodeficiency virus and tuberculosis resource limited setting, gains from Including Xpert MTB/RIF in the diagnostic algorithm of fluid specimens submitted for exclusion of lymphoma by immunophenotypic analysis. PLOS One 2015;10(8):e0134404. [DOI: 10.1371/journal.pone.0134404]

\section{Kim 2014 \{published data only\}}

Kim CH, Woo H, Hyun IG, Kim C, Choi JH, Jang SH, et al. A comparison between the efficiency of the Xpert MTB/RIF assay and nested PCR in identifying Mycobacterium tuberculosis during routine clinical practice. Journal of Thoracic Disease 2014;6(6):625-31.

\section{Kim 2015b \{published data only\}}

Kim MJ, Nam YS, Cho SY, Park TS, Lee HJ. Comparison of the Xpert MTB/RIF Assay and real-time PCR for the detection of Mycobacterium tuberculosis. Annals of Clinical and Laboratory Science 2015;45(3):327-32.

\section{Kim 2015c \{published data only\}}

Kim CH, Hyun IG, Hwang YI, Kim DG, Lee CY, Lee MG, et al. Identification of Mycobacterium tuberculosis and rifampin resistance in clinical specimens using the Xpert MTB/RIF assay. Annals of Clinical and Laboratory Science 2015;45(1):32-8.

\section{Kotovich 2018 \{published data only\}}

Kotovich DS, Skryagina EM, Gurevich GL, Dyusmikeeva MI, Gorenok DI, Golaydo MM. New diagnostic opportunities when testing biological materials from those with pleural effusion caused by tuberculosis. Tuberculosis and Lung Diseases 2018;96(9):5-10.

\section{Koul 2018 \{published data only\}}

Koul AN, Kassana BA, Rather AR. Utility of GeneXpert in the diagnosis, reliance on urine microscopy and clinical characteristics of genitourinary tuberculosis at a tertiary care hospital. Indian Journal of Medical Microbiology 2018;36(1):93-6.

\section{Kumar 2017 \{published data only\}}

Kumar S, Bopanna S, Kedia S, Mouli P, Dhingra R, Padhan R, et al. Evaluation of Xpert MTB/RIF assay performance in the diagnosis of abdominal tuberculosis. Intestinal Research 2017;15(2):187-94

\section{Kumari 2019 \{published data only\}}

Kumari P, Lavania S, Tyagi S, Dhiman A, Rath D, Anthwal D, et al. A novel aptamer-based test for the rapid and accurate diagnosis of pleural tuberculosis. Analytical Biochemistry 2019;564-565:80-7.

\section{Kurbaniyazova 2017 \{published data only\}}

Kurbaniyazova G, Joncevska M, Kalon S, Kalmambetova G, Mohr T, Toktogonova A, et al. Results of Xpert MTB/RIF implementation in Kyrgyzstan. International Journal of Tuberculosis and Lung Disease 2017;21(3):333-7.

Kwak 2015 \{published data only\}

Kwak N, Seong MW, Kim EC, Yoo CG, Kim YW, Han SK, et al. Accuracy of the Xpert MTB/RIF assay for the diagnosis of extrapulmonary tuberculosis in South Korea. International Journal of Tuberculosis and Lung Disease 2015;19(1):81-6.

\section{Lawn 2012 \{published data only\}}

Lawn SD, Kerkhoff AD, Vogt M, Wood R. High diagnostic yield of tuberculosis from screening urine samples from HIV-infected patients with advanced immunodeficiency using the Xpert MTB/ RIF assay. Journal of Acquired Immune Deficiency Syndromes 2012;60(3):289-94.

\section{Lawn 2013 \{published data only\}}

Lawn SD, Kerkhoff AD, Vogt M, Wood R. HIV-associated tuberculosis: relationship between disease severity and the sensitivity of new sputum-based and urine-based diagnostic assays. BMC Medicine 2013;11:231. [DOI: 10.1186/1741-7015-11-231]

\section{Lawn 2015 \{published data only\}}

Lawn SD, Kerkhoff AD, Burton R, Schutz C, Van Wyk G, Vogt M, et al. Rapid microbiological screening for tuberculosis in HIVpositive patients on the first day of acute hospital admission by systematic testing of urine samples using Xpert MTB/RIF: a prospective cohort in South Africa. BMC Medicine 2015;13:192. [DOI: 10.1186/s12916-015-0432-2]

\section{Lawn 2017 \{published data only\}}

Lawn SD, Kerkhoff AD, Burton R, Schutz C, Boulle A, Vogt M, et al. Diagnostic accuracy, incremental yield and prognostic value of Determine TB-LAM for routine diagnostic testing for tuberculosis in HIV-infected patients requiring acute hospital admission in South Africa: a prospective cohort. BMC Medicine 2017;15(1):67. [DOI: 10.1186/s12916-017-0822-8] 


\section{Lee 2017 \{published data only\}}

Lee J, Choi SM, Lee CH, Lee SM, Yim JJ, Yoo CG, et al. The additional role of Xpert MTB/RIF in the diagnosis of intrathoracic tuberculous lymphadenitis. Journal of Infection and Chemotherapy 2017;23(6):381-4.

\section{Lemus-Minor 2018 \{published data only\}}

Lemus-Minor CG, Ovalle-Marroqui DF, Vazquez-Jimenez JG, Reales-Agüero DL, Sepulveda-Alcantara PM, RodriguezSánchez JR, et al. Comparison of the Purelyse - IS6110 nested PCR with the Xpert MTB/RIF test in clinical samples with suspected tuberculosis. Journal of Microbiological Methods 2018;152:48-51.

\section{Li 2018 \{published data only\}}

Li Y, Jia W, Lei G, Zhao D, Wang G, Qin S. Diagnostic efficiency of Xpert MTB/RIF assay for osteoarticular tuberculosis in patients with inflammatory arthritis in China. PLOS One 2018;13(6):e0198600.

\section{Li 2020 \{published data only\}}

Li X, Du W, Wang Y, Liu Z, Li K, Chen H, et al. Rapid diagnosis of tuberculosis meningitis by detecting mycobacterium tuberculosis cell-free DNA in cerebrospinal fluid. American Journal of Clinical Pathology 2020;153(1):126-30.

\section{Liu 2015 \{published data only\}}

Liu X, Huang Z, Du J. Rapid diagnosis of pleural tuberculosis by Xpert MTB/RIF assay. Zhonghua Jie He He Hu Xi Za Zhi [Chinese Journal of Tuberculosis and Respiratory Diseases] 2015;38(10):741-5.

\section{Liu 2019 \{published data only\}}

Liu XH, Xia L, Zhang AM, Zhang Y, Liu YH, Lu SH, et al. Increased diagnostic yield of tuberculous serositis by using serous fluid drainage flocky precipitate (SFDFP) as a testing sample. Scientific Reports 2019;9(1):1480.

\section{Lombardi 2017 \{published data only\}}

Lombardi G, Di Gregori V, Girometti N, Tadolini M, Bisognin F, Dal Monte P. Diagnosis of smear-negative tuberculosis is greatly improved by Xpert MTB/RIF. PLOS One 2017;12(4):e0176186. [DOI: 10.1371/journal.pone.0176186]

\section{Marouane 2014 \{published data only\}}

Marouane C, Smaoui S, Kammoun S, Slim L, MessadiAkrout F. Evaluation of GeneXpert MTB/RIF for the detection of Mycobacterium tuberculosis and resistance to rifampin in extra-pulmonary specimens. International Journal of Mycobacteriology 2014;4 Suppl 1:101.

\section{Marouane 2016 \{published data only\}}

Marouane C, Smaoui S, Kammoun S, Slim L, MessadiAkrout F. Evaluation of molecular detection of extrapulmonary tuberculosis and resistance to rifampicin with GeneXpert(R) MTB/RIF. Medecine et Maladies Infectieuses 2016;46(1):20-4.

\section{Massi 2017 \{published data only\}}

Massi MN, Biatko KT, Handayani I, Pratama MY, Septriani S, Nurdin GM, et al. Evaluation of rapid GeneXpert MTB/RIF method using DNA tissue specimens of vertebral bones in patients with suspected spondylitis TB. Journal of Orthopaedics 2017;14(1):189-91.

Mathew 2018 \{published data only\}

Mathew S, Pradhani MM, Philomina B. Role of molecular diagnostic test in diagnosing pulmonary and extrapulmonary tuberculosis. Journal of Evolution of Medical and Dental Sciences 2018;7(23):2777-81.

Mazzola 2016 \{published data only\}

Mazzola E, Arosio M, Nava A, Fanti D, Gesu G, Farina C. Performance of real-time PCR Xpert (R)MTB/RIF in diagnosing extrapulmonary tuberculosis. Infezioni in Medicina 2016;24(4):304-9.

\section{McMillen 2018 \{published data only\}}

McMillen T, Usiak SC, Chen LH, Gomez L, Ntiamoah P, Hameed MR, et al. Evaluation of the Xpert MTB/RIF performance on tissues: potential impact on airborne infection isolation at a tertiary cancer care center. Infection Control and Hospital Epidemiology 2018;39(4):462-6.

Mechal 2019 \{published data only\} Mechal Y, Benaissa E, El Mrimar N, Benlahlou Y, Bssaibis F, Zegmout A, et al. Evaluation of GeneXpert MTB/RIF system performances in the diagnosis of extrapulmonary tuberculosis. BMC infectious Diseases 2019;19(1):1069.

\section{Metaferia 2018 \{published data only\}}

Metaferia Y, Seid A, Fenta GM, Gebretsadik D. Assessment of extrapulmonary tuberculosis using Gene Xpert MTB/RIF assay and fluorescent microscopy and its risk factors at Dessie Referral Hospital, Northeast Ethiopia. BioMed Research International 2018;2018:8207098.

\section{Miller 2011 \{published data only\}}

Miller MB, Popowitch EB, Backlund MG, Ager EP. Performance of Xpert MTB/RIF RUO assay and IS6110 real-time PCR for Mycobacterium tuberculosis detection in clinical samples. Journal of Clinical Microbiology 2011;49(10):3458-62.

\section{Mishra 2017 \{published data only\}}

Mishra DR, Bhatta N, Lamsal M, Bhattarai N, Maskey R. Study of diagnostic utility of Xpert MTB/RIF test on pleural fluid in the evaluation of patients presenting with pleural tuberculosis in high prevalence setting. American Journal of Respiratory and Critical Care Medicine 2017;195:A2088. [www.atsjournals.org/doi/abs/10.1164/ajrccmconference.2017.195.1_MeetingAbstracts.A2088]

\section{Moure 2011 \{published data only\}}

Moure R, Muñoz L, Torres M, Santin M, Martín R, Alcaide F. Rapid detection of Mycobacterium tuberculosis complex and rifampin resistance in smear-negative clinical samples by use of an integrated real-time PCR method. Journal of Clinical Microbiology 2011;49(3):1137-9.

\section{Moure 2012 \{published data only\}}

Moure R, Martín R, Alcaide F. Effectiveness of an integrated real-time PCR method for detection of the Mycobacterium tuberculosis complex in smear-negative extrapulmonary 
samples in an area of low tuberculosis prevalence. Journal of Clinical Microbiology 2012;50(2):513-5.

\section{Negi 2019 \{published data only\}}

Negi SS, Singh P, Chandrakar S, Gaikwad U, Das P, Bhargava A, et al. Diagnostic evaluation of multiplex real time PCR, genexpert MTB/RIF assay and conventional methods in extrapulmonary tuberculosis. Journal of Clinical and Diagnostic Research 2019;13(1):DC12-6.

\section{Nhu 2013 \{published data only\}}

Nhu NT, Ha DT, Anh ND, Thu DD, Duong TN, Quang ND, et al. Evaluation of Xpert MTB/RIF and MODS assay for the diagnosis of pediatric tuberculosis. BMC Infectious Diseases 2013;13:31. [DOI: 10.1186/1471-2334-13-31]

\section{Omar 2019 \{published data only\}}

Omar A, Elfadl A E, Ahmed Y, Hosny M. Genexpert test and tuberculous pleural effusion: a new diagnostic method for an old medical problem. Egyptian Journal of Chest Diseases and Tuberculosis 2019;68(4):493-7.

\section{Pandey 2017 \{published data only\}}

Pandey S, Congdon J, McInnes B, Pop A, Coulter C. Evaluation of the GeneXpert MTB/RIF assay on extrapulmonary and respiratory samples other than sputum: a low burden country experience. Pathology 2017;49(1):70-4.

\section{Paramitha 2018 \{published data only\}}

Paramitha NA, Sribudiani Y, Rizal A. Comparison of positivity rate of MODS, Ziehl-Neelsen Staining, and GeneXpert Methods in M. tuberculosis detection among tuberculous meningitis patients. Majalah Kedokteran Bandung-Mkb-Bandung Medical Journal 2018;50(4):240-6.

\section{Park 2019 \{published data only\}}

Park JH, Jin CE, Koo B, Kwon JS, Cha HH, Kim JY, et al. A simple microfluidic assay for diagnosing tuberculous meningitis in HIV-uninfected patients. Journal of Clinical Microbiology 2019;57(5):pii: e01975-18.

\section{Patel 2014 \{published data only\}}

Patel VB, Connolly C, Singh R, Lenders L, Matinyenya B, Theron G, et al. Comparison of amplicor and GeneXpert MTB/ RIF tests for diagnosis of tuberculous meningitis. Journal of Clinical Microbiology 2014;52(10):3777-80.

\section{Patel 2020 \{published data only\}}

Patel J, Upadhyay M, Kundnani V, Merchant Z, Jain S, Kire N, et al. Diagnostic efficacy, sensitivity, and specificity of Xpert MTB/RIF assay for spinal tuberculosis and rifampicin resistance. Spine (Phila Pa 1976) 2020;45(3):163-9.

\section{Peter 2012 \{published data only\}}

Peter JG, Theron G, Muchinga TE, Govender U, Dheda K. The diagnostic accuracy of urine-based Xpert MTB/RIF in HIVinfected hospitalized patients who are smear-negative or sputum scarce. PLOS One 2012;7(7):e39966. [DOI: 10.1371/ journal.pone.0039966]

\section{Philip 2017 \{published data only\}}

Philip N, Wilkes C, Yeo TW, Rajahram GS, William T, John DV. Potential use of multiplex PCR in diagnosis of tuberculous meningitis. Tropical Biomedicine 2017;34(4):870-6.

\section{Piersimoni 2019 \{published data only\}}

Piersimoni C, Gherardi G, Gracciotti N, Pocognoli A. Comparative evaluation of Xpert MTB/RIF and the new Xpert MTB/RIF ultra with respiratory and extra-pulmonary specimens for tuberculosis case detection in a low incidence setting. Journal of Clinical Tuberculosis and Other Mycobacterial Diseases 2019;15:100094.

\section{Pink 2016 \{published data only\}}

Pink F, Brown TJ, Kranzer K, Drobniewski F. Evaluation of Xpert MTB/RIF for detection of Mycobacterium tuberculosis in cerebrospinal fluid. Journal of Clinical Microbiology 2016;54(3):809-11.

\section{Pohl 2016 \{published data only\}}

Pohl C, Rutaihwa LK, Haraka F, Nsubuga M, Aloi F, Ntinginya NE, et al. Limited value of whole blood Xpert (R) MTB/RIF for diagnosing tuberculosis in children. Journal of Infection 2016;73(4):326-35.

\section{Porcel 2013 \{published data only\}}

Porcel JM, Palma R, Valdes L, Bielsa S, San-Jose E, Esquerda A. Xpert(R) MTB/RIF in pleural fluid for the diagnosis of tuberculosis. International Journal of Tuberculosis and Lung Disease 2013;17(9):1217-9.

\section{Rachow 2012 \{published data only\}}

Rachow A, Clowes P, Saathoff E, Mtafya B, Michael E, Ntinginya EN, et al. Increased and expedited case detection by Xpert MTB/RIF assay in childhood tuberculosis: a prospective cohort study. Clinical Infectious Diseases 2012;54(10):1388-96.

\section{Raizada 2015 \{published data only\}}

Raizada N, Sachdeva KS, Swaminathan S, Kulsange S, Khaparde SD, Nair SA, et al. Piloting upfront Xpert MTB/RIF testing on various specimens under programmatic conditions for diagnosis of TB \& DR-TB in paediatric population. PLOS One 2015;10(10):e0140375. [DOI: 10.1371/journal.pone.0140375]

\section{Raizada 2018 \{published data only\}}

Raizada N, Khaparde SD, Rao R, Kalra A, Sarin S, Salhotra VS, et al. Upfront Xpert MTB/RIF testing on various specimen types for presumptive infant TB cases for early and appropriate treatment initiation. PLOS One 2018;13(8):e0202085.

Ramamurthy 2016 \{published data only\}

Ramamurthy K, Bhat S, Shenoy S, Rangnekar A. Xpert Mycobacterium tuberculosis/rifampicin assay: a boon in tuberculosis diagnostics. Asian Journal of Pharmaceutical and Clinical Research 2016;9(5):225-7.

Rathour 2019 \{published data only\}

Rathour JS, Mantan M, Khanna A, Hanif M. Evaluation of Gene Xpert assay in extrapulmonary tuberculosis in children. Journal of Evolution of Medical and Dental Sciences 2019;8(1):76-80. 
Razack 2014 \{published data only\}

Razack R, Louw M, Wright CA. Diagnostic yield of fine needle aspiration biopsy in HIV-infected adults with suspected mycobacterial lymphadenitis. South African Medical Journal 2014;104(1):27-8.

\section{Rebecca 2018 \{published data only\}}

Rebecca B, Chacko A, Verghese V, Rose W. Spectrum of pediatric tuberculosis in a tertiary care setting in South India. Journal of Tropical Pediatrics 2018;64(6):544-7.

\section{Reddy 2017 \{published data only\}}

Reddy R, Alvarez-Uria G. Molecular epidemiology of rifampicin resistance in mycobacterium tuberculosis using the GeneXpert MTB/RIF assay from a rural setting in India. Journal of Pathogens 2017;2017:6738095.

\section{Rindi 2017 \{published data only\}}

Rindi L, Ali G, Fabiani B, Fontanini G, Garzelli C. Detection of Mycobacterium tuberculosis from paraffin-embedded tissues by GeneXpert MTB/RIF. Tuberculosis (Edinb) 2017;106:53-5.

\section{Rossato Silva 2018 \{published data only\}}

Rossato Silva D, Sotgiu G, D'Ambrosio L, Rodrigues Pereira G, Silva Barbosa M, Dutra Dias NJ, et al. Diagnostic performances of the Xpert MTB/RIF in Brazil. Respiratory Medicine 2018;134:12-5.

\section{Ruiz 2017 \{published data only\}}

Ruiz P, Causse M, Vaquero M, Gutierrez J B, Casal M. Evaluation of a new automated abbott realtime MTB RIF/INH assay for qualitative detection of rifampicin/isoniazid resistance in pulmonary and extra-pulmonary clinical samples of mycobacterium tuberculosis. Infection and Drug Resistance 2017;10:463-7.

\section{Sachdeva 2018 \{published data only\}}

Sachdeva K, Shrivastava T. CBNAAT: a boon for early diagnosis of tuberculosis-head and neck. Indian Journal of Otolaryngology and Head and Neck Surgery 2018;70(4):572-7.

\section{Saeed 2017a \{published data only\}}

Saeed M, Ahmad M, Iram S, Riaz S, Akhtar M, Aslam M. GeneXpert technology. A breakthrough for the diagnosis of tuberculous pericarditis and pleuritis in less than 2 hours. Saudi Medical Journal 2017;38(7):699-705.

\section{Saeed 2017b \{published data only\}}

Saeed M, Iram S, Hussain S, Ahmed A, Akbar M, Aslam M. GeneXpert: a new tool for the rapid detection of rifampicin resistance in mycobacterium tuberculosis. Journal of the Pakistan Medical Association 2017;67(2):270-4.

\section{Saeed 2018 \{published data only\}}

Saeed M, Rasheed F, Iram S, Hussain S, Ahmad A, Riaz S, et al. False negativity of Ziehl-Neelsen smear microscopy: is the scaleup the [sic] worth it in developing countries? Journal of the College of Physicians and Surgeons--Pakistan 2018;28(3):201-5.

\section{Salvador 2015 \{published data only\}}

Salvador F, Los-Arcos I, Sanchez-Montalva A, Tortola T, Curran A, Villar A, et al. Epidemiology and diagnosis of tuberculous lymphadenitis in a tuberculosis low-burden country. Medicine 2015;94(4):e509. [DOI: 10.1097/MD.0000000000000509]

\section{Samuel 2018 \{published data only\}}

Samuel B, Michael J, Chandrasingh J, Kumar S, Devasia A, Kekre N. Efficacy and role of Xpert Mycobacterium tuberculosis/ rifampicin assay in urinary tuberculosis. Indian Journal of Urology 2018;34(4):268-72.

\section{Sanjuan Jimenez 2015 \{published data only\}}

Sanjuan-Jimenez R, Toro-Peinado I, Bermudez P, Colmenero JD, Morata P. Comparative study of a real-time PCR assay targeting senX3-regX3 versus other molecular strategies commonly used in the diagnosis of tuberculosis. PLOS One 2015;10(11):e0143025. [DOI: 10.1371/journal.pone.0143025]

\section{Schutz 2019 \{published data only\}}

Schutz C, Ward A, Burton R, Nicol M P, Blumenthal L, Meintjes G, et al. False rifampicin resistant results using Xpert MTB/RIF on urine samples in hospitalised HIV-infected patients. Southern African Journal of HIV Medicine 2019;20(1):978.

\section{Sekyere 2019 \{published data only\}}

Osei Sekyere J, Maphalala N, Malinga LA, Mbelle NM, Maningi NE. A comparative evaluation of the new Genexpert MTB/RIF Ultra and other rapid diagnostic assays for detecting tuberculosis in pulmonary and extra pulmonary specimens. Scientific Reports 2019;9(1):16587.

\section{Set 2018 \{published data only\}}

Set R, Bankar S, Sharma D, Shah D, Shastri J. Performance of Xpert MTB/RIF for detection of Mycobacterium tuberculosis and rifampicin resistance in pus aspirates. Indian Journal of Tuberculosis 2018;66(4):433-6.

\section{Set 2019 \{published data only\}}

Set R, Bankar S, Sharma D, Shah D, Shastri J. Performance of Xpert MTB/RIF for detection of Mycobacterium tuberculosis and rifampicin resistance in pus aspirates. Indian Journal of Tuberculosis 2019;66(4):433-6.

\section{Shah 2016a \{published data only\}}

Shah I, Gupta Y, Chhina AS, Shenoi A, Kumar RK, Patel A. Xpert MTB/RIF for diagnosis of tuberculosis and drug resistance in Indian children. Indian Pediatrics 2016;53(9):837-9.

\section{Shakeel 2018 \{published data only\}}

Shakeel K, Iram S, Akhtar M, Hussain S, Maryam H, Anwar A Diagnostic validation of rapid molecular detection of Mycobacterium tuberculosis in pus samples by GeneXpert. Journal of the Pakistan Medical Association 2018;68(1):33-7.

\section{Sharma 2017a \{published data only\}}

Sharma SK, Chaubey J, Singh BK, Sharma R, Mittal A, Sharma A. Drug resistance patterns among extra-pulmonary tuberculosis cases in a tertiary care centre in North India. International Journal of Tuberculosis and Lung Disease 2017;21(10):1112-7. 
Sharma 2019 \{published data only\}

Sharma K, Gupta A, Sharma M, Sharma A, Bansal R, Sharma SP, et al. The emerging challenge of diagnosing drug-resistant tubercular uveitis: experience of 110 eyes from North India. Ocular Immunology and Inflammation 2019;27:1-8.

\section{Singanayagam 2014 \{published data only\}}

Singanayagam A, Donaldson H, Kon OM. GeneXpert ${ }^{\mathrm{TM} M T B / R I F}$ in low prevalence settings: a UK laboratory perspective. Journal of Infection 2014;69(2):199-200.

\section{Singh 2016 \{published data only\}}

Singh UB, Pandey P, Mehta G, Bhatnagar AK, Mohan A, Goyal V, et al. Genotypic, phenotypic and clinical validation of GeneXpert in extra-pulmonary and pulmonary tuberculosis in India. PLOS One 2016;11(2):e0149258. [DOI: 10.1371/ journal.pone.0149258]

\section{Smith 2014 \{published data only\}}

Smith P, Van Esch A, Wallace M, Wood R, Bekker LG. GeneXpert TB 8: a point-of-care diagnostic pilot. South African Medical Journal 2014;104(8):524.

\section{Solomons 2015 \{published data only\}}

Solomons RS, Visser DH, Friedrich SO, Diacon AH, Hoek KG, Marais BJ, et al. Improved diagnosis of childhood tuberculous meningitis using more than one nucleic acid amplification test. International Journal of Tuberculosis and Lung Disease 2015;19(1):74-80.

\section{Solomons 2016 \{published data only\}}

Solomons RS, Visser DH, Marais BJ, Schoeman JF, Van Furth AM. Diagnostic accuracy of a uniform research case definition for TBM in children: a prospective study. International Journal of Tuberculosis and Lung Disease 2016;20(7):903-8.

\section{Soomro 2017 \{published data only\}}

Soomro NH, Baig S, Rao N, Zafar AA, Hashmat F, Awan SB, et al. GeneXpert of cervical lymph node specimens in the diagnosis of extrapulmonary TB and rifampicin resistance. Gomal Journal of Medical Sciences 2017;15(1):12-5.

\section{Sumayya 2019 \{published data only\}}

Sumayya, Durga K, Nithyananda BS, Fatima S, Krishnaiah A. Study of CBNAAT and anti-MPT64 detection in cytological and histopathological material for early diagnosis of TB lymphadenitis. Journal of Evolution of Medical and Dental Sciences 2019;8(47):3540-4

\section{Tahseen 2019 \{published data only\}}

Tahseen S, Ambreen A, Masood F, Qadir M, Hussain A, Jamil M, et al. Primary drug resistance in extra-pulmonary tuberculosis: a hospital-based prospective study from Pakistan. International Journal of Tuberculosis and Lung Diseases 2019;23(8):900-6.

\section{Talib 2019 \{published data only\}}

Talib A, Bhatty S, Mehmood K, Naim H, Haider I, Lal H, et al. GeneXpert in stool: diagnostic yield in intestinal tuberculosis. Journal of Clinical Tuberculosis and Mycobacterial Diseases 2019;17:100131.
Tang 2017 \{published data only\}

Tang L, Feng S, Gao R, Han C, Sun X, Bao Y, et al. A comparative study on the role of Xpert MTB/RIF in testing different types of spinal tuberculosis tissue specimens. Genetic Testing and Molecular Biomarkers 2017;21(12):722-6.

Tang 2018 \{published data only\}

Tang Y, Yin L, Tang S, Zhang H, Lan J. Application of molecular, microbiological, and immunological tests for the diagnosis of bone and joint tuberculosis. Journal of Clinical Laboratory Analysis 2018;32(2):e22260. [DOI: 10.1002/jcla.22260]

Teo 2011 \{published data only\}

Teo J, Jureen R, Chiang D, Chan D, Lin R. Comparison of two nucleic acid amplification assays, the Xpert MTB/RIF assay and the amplified mycobacterium tuberculosis direct assay, for detection of Mycobacterium tuberculosis in respiratory and nonrespiratory specimens. Journal of Clinical Microbiology 2011;49(10):3659-62

\section{Terzi 2019 \{published data only\}}

Terzi HA, Aydemir O, Karakece E, Koroglu M, Altindis M. Comparison of the GeneXpert(R) MTB/RIF test and conventional methods in the diagnosis of Mycobacterium tuberculosis. Clinical Laboratory 2019;65(1):10.7754/Clin.Lab.2018.180613. [DOI: 10.7754/Clin.Lab.2018.180613]

Theron 2014b \{published data only\}

Theron G, Peter J, Calligaro G, Meldau R, Hanrahan C, Khalfey H, et al. Determinants of PCR performance (Xpert MTB/RIF), including bacterial load and inhibition, for TB diagnosis using specimens from different body compartments. Scientific Reports 2014;4:5658. [DOI: 10.1038/srep05658]

\section{Tortoli 2012 \{published data only\}}

Tortoli E, Russo C, Piersimoni C, Mazzola E, Dal Monte P, Pascarella M, et al. Clinical validation of Xpert MTB/RIF for the diagnosis of extrapulmonary tuberculosis. European Respiratory Journal 2012;40(2):442-7.

\section{Toure 2017 \{published data only\}}

Toure NO, Wayzani M, Thiam K, Cisse MF, Mbaye FB. Contribution of the Xpert MTB/RIF to the etiological diagnosis of tuberculous pleurisy [Apport de l'Xpert MTB/RIF dans le diagnostic étiologique des pleurésies tuberculeuses]. Revue des Maladies Respiratoires 2017;34(7):758-64. [DOI: 10.1016/ j.rmr.2017.01.003]

\section{Uddin 2019 \{published data only\}}

Uddin MJ, Rahim MA, Hasan MN, Mazumder MK, Haq MM, Rahman MA, et al. Etiological evaluation of patients with lymphadenopathy by clinical, histopathological and microbiological assessment. Mymensingh Medical Journal 2019;28(4):854-61.

\section{Vallejo 2015 \{published data only\}}

Vallejo VP, Searle MA, Rodriguez DJ, Farga CV. Xpert ${ }^{\circledR}$ MTB/RIF assay for tuberculosis diagnosis [Ensayo Xpert ${ }^{\circledR}$ MTB/RIF en el diagnóstico de tuberculosis]. Revista Chilena de Enfermedades Respiratorias 2015;31(2):127-31. 


\section{Verghese 2016 \{published data only\}}

Verghese VP, Thomas L, Michael JS, Rose W, Jeyaseelan V. Accuracy of the Xpert MTB/RIF assay compared to the "gold standard" AFB culture in the diagnosis of tuberculosis in children in India. International Journal of Infectious Diseases 2016;45:343.

\section{Wang 2016a \{published data only\}}

Wang T, Feng GD, Pang Y, Liu JY, Zhou Y, Yang YN, et al. High rate of drug resistance among tuberculous meningitis cases in Shaanxi province, China. Scientific Reports 2016;6:25251. [DOI: 10.1038/srep25251]

\section{Wang 2016b \{published data only\}}

Wang T, Feng GD, Pang Y, Yang YN, Dai W, Zhang L, et al. Suboptimal specificity of modified Ziehl-Neelsen staining for quick identification of tuberculous meningitis. Frontiers in Microbiology 2016;7:2096-103.

\section{Wang 2018 \{published data only\}}

Wang G, Dong W, Lan T, Fan J, Tang K, Li Y, et al. Diagnostic accuracy evaluation of the conventional and molecular tests for spinal tuberculosis in a cohort, head-to-head study. Emerging Microbes \& Infections 2018;7(1):109.

\section{Wei 2016 \{published data only\}}

Wei G, Mu J, Wang G, Huo F, Dong L, Li Y, et al. The reliability analysis of Xpert-positive result for smear-negative and culturenegative specimen collected from bone and joint tuberculosis suspects. Journal of Thoracic Disease 2016;8(6):1205-9.

\section{Yang 2017 \{published data only\}}

Yang CQ, Liu XY, Du RH, Cao TZ, Dai XY. Diagnosis value with Xpert Mtb/RIF assay for cervical tuberculous lymphadenitis. Lin Chung Er Bi Yan Hou Tou Jing Wai Ke Za Zhi 2017;31(17):1338-40.

Yang 2019 \{published data only\}

Yang X, Che N, Duan H, Liu Z, Li K, Li H, et al. Cell-free Mycobacterium tuberculosis DNA test in pleural effusion for tuberculous pleurisy: a diagnostic accuracy study. Clinical Microbiology and Infection 2019;8:1089.e1-1089.e6.

\section{Yu 2020 \{published data only\}}

Yu G, Shen Y, Ye B, Chen D, Xu K. Comparison of CapitalBio Mycobacterium nucleic acid detection test and Xpert MTB/ RIF assay for rapid diagnosis of extrapulmonary tuberculosis. Journal of Microbiological Methods 2020;168:105780.

Yuan 2016 \{published data only\}

Yuan M, Lyu Y, Chen ST, Cai C, Li Y, Zhang ZG, et al. Evaluation of Xpert MTB/RIF for the diagnosis of extrapulmonary tuberculosis in China. Biomedical and Environmental Sciences 2016;29(8):599-602.

\section{Zhang 2016 \{published data only\}}

Zhang AM, Li F, Liu XH, Xia L, Lu SH. Application of Gene Xpert Mycobacterium tuberculosis DNA and resistance to rifampicin assay in the rapid detection of tuberculosis in children. Zhonghua Er Ke Za Zhi [Chinese Journal of Pediatrics] 2016;54(5):370-4.

\section{Zhou 2020 \{published data only\}}

Zhou Z, Zheng Y, Wang L. A comparative study on the value of Xpert MTB/RIF and T-SPOT.TB tests in the diagnosis of bone and joint tuberculosis. Clinica Chimica Acta 2020;500:115-9.

\section{Zurcher 2019 \{published data only\}}

Zurcher K, Ballif M, Kiertiburanakul S, Chenal H, Yotebieng M, Grinsztejn B, et al. Diagnosis and clinical outcomes of extrapulmonary tuberculosis in antiretroviral therapy programmes in low- and middle-income countries: a multicohort study. Journal of the International AIDS Society 2019;22(9):e25392.

\section{Additional references}

\section{American Thoracic Society 2000}

American Thoracic Society, the Centers for Disease Control and Prevention, Infectious Disease Society of America. Diagnostic Standards and Classification of Tuberculosis in Adults and Children. This official statement of the American Thoracic Society and the Centers for Disease Control and Prevention was adopted by the ATS Board of Directors, July 1999. This statement was endorsed by the Council of the Infectious Disease Society of America, September 1999. American Journal Respiratory and Critical Care Medicine 2000;161(4 Pt 1):1376-95.

\section{Bahr 2016}

Bahr NC, Marais S, Caws M, Van Crevel R, Wilkinson RJ, Tyagi JS, et al, Tuberculous Meningitis International Research Consortium. GeneXpert MTB/Rif to diagnose tuberculous meningitis: perhaps the first test but not the last. Clinical Infectious Diseases 2016;62(9):1133-5.

\section{Balshem 2011}

Balshem H, Helfand M, Schünemann HJ, Oxman AD, Kunz R, Brozek J, et al. GRADE guidelines: 3. Rating the quality of evidence. Journal of Clinical Epidemiology 2011;64(4):401-6.

\section{Banada 2010}

Banada PP, Sivasubramani SK, Blakemore R, Boehme C, Perkins MD, Fennelly K, et al. Containment of bioaerosol infection risk by the Xpert MTB/RIF assay and its applicability to point-of-care settings. Journal of Clinical Microbiology 2010;48(10):3551-7.

\section{Begg 1983}

Begg CB, Greenes RA. Assessment of diagnostic tests when disease verification is subject to selection bias. Biometrics 1983;39(1):207-15.

\section{Bjerrum 2019}

Bjerrum S, Schiller I, Dendukuri N, Kohli M, Nathavitharana RR, Zwerling AA, et al. Lateral flow urine lipoarabinomannan assay for detecting active tuberculosis in people living with HIV. Cochrane Database of Systematic Reviews 2019, Issue 10. Art. No: CD011420. [DOI: 10.1002/14651858.CD011420.pub3] 


\section{Blakemore 2010}

Blakemore R, Story E, Helb D, Kop J, Banada P, Owens MR, et al. Evaluation of the analytical performance of the Xpert MTB/RIF assay. Journal of Clinical Microbiology 2010;48(7):2495-501.

\section{Boehme 2010}

Boehme CC, Nabeta P, Hillemann D, Nicol MP, Shenai S, Krapp F, et al. Rapid molecular detection of tuberculosis and rifampin resistance. New England Journal of Medicine 2010;363(11):100515.

\section{Bossuyt 2015}

Bossuyt PM, Reitsma JB, Bruns DE, Gatsonis CA, Glasziou PP, Irwig L, et al. STARD 2015: an updated list of essential items for reporting diagnostic accuracy studies. BMJ 2015;351:h5527. [DOI: 10.1136/bmj.h5527]

\section{Branigan 2019}

Branigan D. Pipeline report 2019 tuberculosis diagnostics. www.treatmentactiongroup.org/wp-content/uploads/2019/12/ pipeline_tb_diagnotics_2019_db_final.pdf (accessed 4 April 2020).

\section{Broger 2020}

Broger T, Nicol MP, Székely R, Bjerrum S, Sossen B, Schutz C, et al. Diagnostic accuracy of a novel tuberculosis point-of-care urine lipoarabinomannan assay for people living with HIV: a meta-analysis of individual in- and outpatient data. PLOS Medicine 2020;17(5):e1003113.

\section{CDC 2018}

Centers for Disease Control and Prevention. Reported tuberculosis in the United States, 2018. www.cdc.gov/tb/ statistics/reports/2018/table15.htm (accessed 29 June 2020).

\section{Cepheid 2018}

Cepheid. Brochure: Xpert ${ }^{\circledR}$ MTB/RIF Ultra. www.cepheid.com/ en/tests/Critical-Infectious-Diseases/Xpert-MTB-RIF-Ultra (accessed 26 March 2020).

\section{Cepheid 2019}

Cepheid. Xpert ${ }^{\circledR}$ MTB/RIF. Two-hour detection of MTB and rifampin resistance mutations. www.cepheid.com/Package \%20Insert\%20Files/Xpert-MTB-RIF-ENGLISH-PackageInsert-301-1404-Rev-F.pdf (accessed 29 March 2020).

\section{Chakravorty 2017}

Chakravorty S, Simmons AM, Rowneki M, Parmar H, Cao Y, Ryan J, et al. The new Xpert MTB/RIF Ultra: improving detection of Mycobacterium tuberculosis and resistance to rifampin in an assay suitable for point-of-care testing. MBio 2017;8(4):e00812-17. [DOI: 10.1128/mBio.00812-17]

\section{Chang 2012}

Chang K, Lu W, Wang J, Zhang K, Jia S, Li F, et al. Rapid and effective diagnosis of tuberculosis and rifampicin resistance with Xpert MTB/RIF assay: a meta-analysis. Journal of Infection 2012;64(6):580-8.

\section{Chin 2019a}

Chin JH, Ssengooba W, Grossman S, Pellinen J, Wadda V. Xpert MTB/RIF Ultra: optimal procedures for the detection of Mycobacterium tuberculosis in cerebrospinal fluid. Journal of Clinical Tuberculosis and Other Mycobactaterial Diseases 2019;14:16-18.

\section{Chow 2002}

Chow KM, Chow VC, Hung LC, Wong SM, Szeto CC. Tuberculous peritonitis-associated mortality is high among patients waiting for the results of mycobacterial cultures of ascitic fluid samples. Clinical Infectious Diseases 2002;35(4):409-13.

\section{Chu 2006}

Chu H, Cole SR. Bivariate meta-analysis of sensitivity and specificity with sparse data: a generalized linear mixed model approach. Journal of Clinical Epidemiology 2006;59(12):1331-2.

\section{Conde 2003}

Conde MB, Loivos AC, Rezende VM, Soares SL, Mello FC, Reingold AL, et al. Yield of sputum induction in the diagnosis of pleural tuberculosis. American Journal of Respiratory and Critical Care Medicine 2003;167(5):723-5.

\section{Covidence 2017 [Computer program]}

Veritas Health Innovation Covidence systematic review software. Available at www.covidence.org. Melbourne: Veritas Health Innovation, 2017.

\section{Denkinger 2014}

Denkinger CM, Schumacher SG, Boehme CC, Dendukuri N, Pai M, Steingart KR. Xpert MTB/RIF assay for the diagnosis of extrapulmonary tuberculosis: a systematic review and metaanalysis. European Respiratory Journal 2014;44(2):435-46.

\section{Diacon 2003}

Diacon AH, Van de Wal BW, Wyser C, Smedema JP, Bezuidenhout J, Bolliger CT, et al. Diagnostic tools in tuberculous pleurisy: a direct comparative study. European Respiratory Journal 2003;22(4):589-91.

\section{Dinnes 2007}

Dinnes J, Deeks J, Kunst H, Gibson A, Cummins E, Waugh N, et al. A systematic review of rapid diagnostic tests for the detection of tuberculosis infection. Health Technology Assessment 2007;11(3):1-196.

\section{Di Tanna 2019}

Di Tanna GL, Khaki AR, Theron G, McCarthy K, Cox H, Mupfumi L, et al. Effect of Xpert MTB/RIF on clinical outcomes in routine care settings: individual patient data meta-analysis. Lancet Global Health 2019;7:e191-99.

\section{Dorman 2018}

Dorman SE, Schumacher SG, Alland D, Nabeta P, Armstrong DT, King B, et al. Xpert MTB/RIF Ultra for detection of Mycobacterium tuberculosis and rifampicin resistance: a prospective multicentre diagnostic accuracy study. Lancet Infectious Diseases 2018;18(1):76-84. 


\section{Drain 2019}

Drain PK, Gardiner J, Hannah H, Broger T, Dheda K, Fielding K, et al. Guidance for studies evaluating the accuracy of biomarkerbased nonsputum tests to diagnose tuberculosis. Journal of Infectious Diseases 2019;220(Suppl 3):S108-S115.

\section{FIND 2017}

Foundation for Innovative New Diagnsotics (FIND). Report for WHO: a multicentre non-inferiority diagnostic accuracy study of the Ultra assay compared to the Xpert MTB/RIF assay. www.finddx.org/publication/ultra-report/ (accessed 2 July 2020);(version 1.8):1-82.

\section{Fontanilla 2011}

Fontanilla JM, Barnes A, Von Reyn CF. Current diagnosis and management of peripheral tuberculous lymphadenitis. Clinical Infectious Diseases 2011;53(6):555-62.

\section{Global Laboratory Initiative 2017}

Global Laboratory Initiative. Planning for country transition to Xpert ${ }^{\circledR}$ MTB/RIF Ultra cartridges. www.stoptb.org/wg/gli/assets/ documents/gli_ultra.pdf (accessed 2 July 2020).

\section{Global Laboratory Initiative 2019}

Global Laboratory Intiaitive. Practical guide to implementing a quality assurance system for Xpert MTB/RIF testing. www.stoptb.org/wg/gli/assets/documents/Xpert-QAguide-2019.pdf (accessed 7 January 2020).

\section{Golden 2005}

Golden MP, Vikram HR. Extrapulmonary tuberculosis: an overview. American Family Physician 2005;72(9):1761-8.

\section{Gopinath 2010}

Gopinath K, Singh S. Non-tuberculous mycobacteria in TBendemic countries: are we neglecting the danger? PLOS Neglected Tropical Diseases 2010;4(4):e615. [DOI: 10.1371/ journal.pntd.0000615]

\section{GRADEpro GDT 2015 [Computer program]}

McMaster University (developed by Evidence Prime) GRADEpro GDT. Version accessed 2 July 2020. Hamilton (ON): McMaster University (developed by Evidence Prime), 2015. Available at gradepro.org.

\section{Gupta 2015}

Gupta RK, Lucas SB, Fielding KL, Lawn SD. Prevalence of tuberculosis in post-mortem studies of HIV-infected adults and children in resource-limited settings: a systematic review and meta-analysis. AIDS 2015;29(15):1987-2002.

\section{Gupta 2018}

Gupta R, Talwar P, Talwar P, Khurana S, Kushwaha S, Jalan N, et al. Diagnostic accuracy of nucleic acid amplification based assays for tuberculous meningitis: a meta-analysis. Journal of Infection 2018;77(4):302-13.

\section{Gupta-Wright 2018}

Gupta-Wright A, Corbett EL, Van Oosterhout JJ, Wilson D, Grint D, Alufandika-Moyo M, et al. Rapid urine-based screening for tuberculosis in HIV-positive patients admitted to hospital in Africa (STAMP): a pragmatic, multicentre, parallelgroup, double-blind, randomised controlled trial. Lancet 2018;392(10144):292-301. [PMID: 30032978]

\section{Haraka 2018}

Haraka F, Nathavitharana RR, Schumacher SG, Kakolwa M, Denkinger CM, Gagneux S, et al. Impact of diagnostic test Xpert MTB/RIF ${ }^{\circledR}$ on health outcomes for tuberculosis. Cochrane Database of Systematic Reviews 2018, Issue 2. Art. No: CD012972. [DOI: 10.1002/14651858.CD012972]

\section{Harbord 2007}

Harbord RM, Deeks JJ, Egger M, Whiting P, Sterne JA. A unification of models for meta-analysis of diagnostic accuracy studies. Biostatistics 2007;8(2):239-51.

\section{Hartmann 1967}

Hartmann G, Honikel KO, Knüsel F, Nüesch J. The specific inhibition of the DNA-directed RNA synthesis by rifamycin. Biochimica et Biophysica Acta 1967;145(3):843-4.

\section{Helb 2010}

Helb D, Jones M, Story E, Boehme C, Wallace E, Ho K, et al. Rapid detection of Mycobacterium tuberculosis and rifampin resistance by use of on-demand, near-patient technology. Journal of Clinical Microbiology 2010;48(1):229-37.

\section{Horne 2019}

Horne DJ, Kohli M, Zifodya JS, Schiller I, Dendukuri N, Tollefson D, et al. Xpert MTB/RIF and Xpert MTB/RIF Ultra for pulmonary tuberculosis and rifampicin resistance in adults. Cochrane Database of Systematic Reviews 2019, Issue 6. Art. No: CD009593. [DOI: 10.1002/14651858.CD009593]

\section{Iseman 2000}

Iseman MD. Extrapulmonary tuberculosis in adults. In: Iseman MD, editors(s). A Clinician's Guide to Tuberculosis. Philadelphia: Lippincott Williams and Wilkins, 2000:145-97.

\section{Kay 2020}

Kay AW, González Fernández L, Takwoingi Y, Eisenhut M, Vu RD, Steingart KR, et al. Xpert MTB/RIF and Xpert MTB/RIF Ultra assays for active tuberculosis and rifampicin resistance in children. Cochrane Database of Systematic Reviews 2020, Issue 8. Art. No: CD013359. [DOI: 10.1002/14651858.CD013359] [PMID: 32853411]

\section{Kilpatrick 1986}

Kilpatrick ME, Girgis NI, Yassin MW, Abu el Ella AA. Tuberculous meningitis-clinical and laboratory review of 100 patients. Journal of Hygiene (London) 1986;96(2):231-8.

\section{Leeflang 2013}

Leeflang MM, Rutjes AW, Reitsma JB, Hooft L, Bossuyt PM. Variation of a test's sensitivity and specificity with disease prevalence. Canadian Medical Association Journal 2013;185(11):E537-44. [DOI: 10.1503/cmaj.121286]

\section{Lewinsohn 2017}

Lewinsohn DM, Leonard MK, LoBue PA, Cohn DL, Daley CL, Desmond E, et al. Official American Thoracic Society/Infectious 
Diseases Society of America/Centers for Disease Control and Prevention Clinical Practice Guidelines: Diagnosis of Tuberculosis in Adults and Children. Clinical Infectious Diseases 2017;64(2):e1-33. [DOI: 10.1093/cid/ciw694]

\section{Li Y 2017}

Li S, Liu B, Peng M, Chen M, Yin W, Tang H, et al. Diagnostic accuracy of Xpert MTB/RIF for tuberculosis detection in different regions with different endemic burden: a systematic review and meta-analysis. PLOS One 2017;12(7):e0180725. [DOI: 10.1371/ journal.pone.0180725]

\section{Lunn 2009}

Lunn D, Spiegelhalter D, Thomas A, Best N. The BUGS project: evolution, critique, and future directions. Statistics in Medicine 2009;28(25):3049-67.

\section{Macaskill 2010}

Macaskill P, Gatsonis C, Deeks JJ, Harbord RM, Takwoingi Y. Chapter 10: Analysing and presenting results. In: Deeks JJ, Bossuyt PM, C Gatsonis (editors). Cochrane Handbook for Systematic Reviews of Diagnostic Test Accuracy Version 1.0. The Cochrane Collaboration, 2010. Available at: methods.cochrane.org/sdt/handbook-dta-reviews.

\section{Marais 2010}

Marais S, Thwaites G, Schoeman JF, Török ME, Misra UK, Prasad K, et al. Tuberculous meningitis: a uniform case definition for use in clinical research. Lancet Infectious Diseases 2010;10(11):803-12.

\section{Maynard-Smith 2014}

Maynard-Smith L, Larke N, Peters JA, Lawn SD. Diagnostic accuracy of the Xpert MTB/RIF assay for extrapulmonary and pulmonary tuberculosis when testing non-respiratory samples: a systematic review. BMC Infectious Diseases 2014;14:709. [DOI: 10.1186/s12879-014-0709-7]

\section{McInnes 2018}

McInnes MD, Moher D, Thombs BD, McGrath TA, Bossuyt PM, et al, anad the PRISMA-DTA Group. Preferred Reporting Items for a Systematic Review and Meta-analysis of Diagnostic Test Accuracy Studies: the PRISMA-DTA statement [published correction appears in JAMA. 2019 Nov 26;322(20):2026]. JAMA 2018;319(4):388-96. [DOI: 10.1001/jama.2017.19163]

\section{Miotto 2012}

Miotto P, Bigoni S, Migliori GB, Matteelli A, Cirillo DM. Early tuberculosis treatment monitoring by Xpert(R) MTB/RIF. European Respiratory Journal 2012;39(5):1269-71.

\section{Moher 2009}

Moher D, Liberati A, Tetzlaff J, Altman DG, The PRISMA Group (2009). Preferred reporting items for systematic reviews and meta-analyses: the PRISMA Statement. PLOS Medicine 6;7:e1000097. [DOI: 10.1371/journal.pmed1000097]

\section{Naaktgeboren 2013}

Naaktgeboren CA, Bertens LC, Van Smeden M, De Groot JA, Moons KG, Reitsma JB. Value of composite reference standards in diagnostic research. BMJ 2013;347:f5605.

\section{Nahid 2016}

Nahid P, Dorman SE, Alipanah N, Barry PM, Brozek JL, Cattamanchi A, et al. Official American Thoracic Society/ Centers for Disease Control and Prevention/Infectious Diseases Society of America clinical practice guidelines: treatment of drug-susceptible tuberculosis. Clinical Infectious Diseases 2016;63(7):e147-95. [DOI: 10.1093/cid/ciw376]

\section{Nathavitharana 2017}

Nathavitharana RR, Cudahy PG, Schumacher SG, Steingart KR, Pai M, Denkinger CM. Accuracy of line probe assays for the diagnosis of pulmonary and multidrug-resistant tuberculosis: a systematic review and meta-analysis. European Respiratory Journal 2017;49(1):pii: 1601075.

\section{Nelson 2004}

Nelson LJ, Wells CD. Global epidemiology of childhood tuberculosis. International Journal of Tuberculosis and Lung Disease 2004;8(5):636-47.

\section{Pai 2004}

Pai M, Flores LL, Hubbard A, Riley LW, Colford JM Jr. Nucleic acid amplification tests in the diagnosis of tuberculous pleuritis: a systematic review and meta-analysis. BMC Infectious Diseases 2004;4:6. [DOI: 10.1186/1471-2334-4-6]

\section{Pai 2008}

Pai M, Ling DI. Rapid diagnosis of extrapulmonary tuberculosis using nucleic acid amplification tests: what is the evidence? Future Microbiology 2008;3(1):1-4.

\section{Pai 2016}

Pai M, Behr M, Dowdy D, Dheda K, Divangahi M, Boehme CC, et al. Tuberculosis. Nature Reviews. Disease Primers 2016;2:16076. [DOI: 10.1038/nrdp.2016.76]

\section{Pang 2019}

Pang Y, An J, Shu W, Huo F, Chu N, Gao M, et al. Epidemiology of extrapulmonary tuberculosis among inpatients, China, 2008-2017. Emerging Infectious Diseases 2019;25:457-64.

\section{Penz 2015}

Penz E, Boffa J, Roberts DJ, Fisher D, Cooper R, Ronksley PE, et al. Diagnostic accuracy of the Xpert ${ }^{\circledR}$ MTB/RIF assay for extrapulmonary tuberculosis: a meta-analysis. International Journal of Tuberculosis and Lung Disease 2015;19(3):278-84.

\section{Perkins 2007}

Perkins MD, Cunningham J. Facing the crisis: improving the diagnosis of tuberculosis in the HIV era. Journal of Infectious Diseases 2007;196 Suppl 1:S15-27.

\section{Peter 2016}

Peter JG, Zijenah LS, Chanda D, Clowes P, Lesosky M, Gina P, et al. Effect on mortality of point-of-care, urine-based lipoarabinomannan testing to guide tuberculosis treatment initiation in HIV-positive hospital inpatients: a pragmatic, parallel-group, multicountry, open-label, randomised controlled trial. Lancet 2016;387(10024):1187-97. 


\section{Pormohammad 2019}

Pormohammad A, Nasiri MJ, McHugh TD, Riahi SM, Bahr NC. A systematic review and meta-analysis of the diagnostic accuracy of nucleic acid amplification tests for tuberculous meningitis. Journal of Clinical Microbiology 2019;57(6):e01113-18.

\section{R Core Team 2019 [Computer program]}

R Foundation for Statistical Computing R Core Team (2019). R: A language and environment for statistical computing. Version accessed after 12 June 2020. Vienna, Austria: R Foundation for Statistical Computing, 2019. Available at r-project.org/.

\section{Reitsma 2005}

Reitsma JB, Glas AS, Rutjes AW, Scholten RJ, Bossuyt PM, Zwinderman $\mathrm{AH}$. Bivariate analysis of sensitivity and specificity produces informative summary measures in diagnostic reviews. Journal of Clinical Epidemiology 2005;58(10):982-90.

\section{Reuter 2009}

Reuter H, Wood R, Schaaf HS, Donald PR. Overview of extrapulmonary tuberculosis in adults and children. In: Schaaf HS, Zumla A, editors(s). Tuberculosis: A Comprehensive Clinical Reference. 1st edition. Amsterdam: Elsevier Science Publishers, 2009:377-90.

\section{RevMan 2014 [Computer program]}

Nordic Cochrane Centre, The Cochrane Collaboration Review Manager 5 (RevMan 5). Version 5.3. Copenhagen: Nordic Cochrane Centre, The Cochrane Collaboration, 2014.

\section{Sandgren 2013}

Sandgren A, Hollo V, Van der Werf MJ. Extrapulmonary tuberculosis in the European Union and European economic area, 2002 to 2011. Euro Surveillance 2013;18(12):pii: 20431.

\section{Schiller 2016}

Schiller I, Van Smeden M, Hadgu A, Libman M, Reitsma JB, Dendukuri N. Bias due to composite reference standards in diagnostic accuracy studies. Statistics in Medicine 2016;35(9):1454-70.

\section{Schumacher 2016}

Schumacher S, Van Smeden M, Dendukuri N, Joseph L, Nicol M, Pai M, et al. Diagnostic test accuracy in childhood pulmonary tuberculosis: a Bayesian latent class analysis. American Journal of Epidemiology 2016;184(9):690-700.

\section{Schumacher 2019}

Schumacher SG, Denkinger CM. The impact of Xpert MTB/RIFdo we have a final answer? Lancet Global Health 2019;7(2):e161e162.

\section{Schünemann 2008}

Schünemann HJ, Oxman AD, Brozek J, Glasziou P, Jaeschke R, Vist GE, et al. Grading quality of evidence and strength of recommendations for diagnostic tests and strategies. $B M \mathrm{~J}$ 2008;336(7653):1106-10.

\section{Schünemann 2016}

Schünemann HJ, Mustafa R, Brozek J, Santesso N, AlonsoCoello P, Guyatt G, et al. GRADE Working Group. GRADE
Guidelines: 16. GRADE evidence to decision frameworks for tests in clinical practice and public health. Journal of Clinical Epidemiology 2016;76:89-98. [DOI: 10.1016/ j.jclinepi.2016.01.032]

\section{Schünemann 2020a}

Schünemann HJ, Mustafa R, Brozek J, Steingart KR, Leeflang M, Murad $\mathrm{MH}$, et al. GRADE guidelines: 21 part 1. Study design, risk of bias and indirectness in rating the certainty across a body of evidence for test accuracy. Journal of Clinical Epidemiology 2020;122:129-41. [DOI: 10.1016/j.jclinepi.2019.12.020]

\section{Schünemann 2020b}

Schünemann HJ, Mustafa R, Brozek J, Steingart KR, Leeflang M, Murad MH, et al. GRADE guidelines: 21 part 2. Inconsistency, Imprecision, publication bias and other domains for rating the certainty of evidence for test accuracy and presenting it in evidence profiles and summary of findings tables. Journal of Clinical Epidemiology 2020;122:142-52. [DOI: 10.1016/ j.jclinepi.2019.12.021]

\section{Sehgal 2016}

Sehgal IS, Dhooria S, Aggarwal AN, Behera D, Agarwal R. Diagnostic performance of Xpert MTB/RIF in tuberculous pleural effusion: systematic review and meta-analysis. Journal of Clinical Microbiology 2016;54(4):1133-6.

\section{Shah 2016b}

Shah M, Hanrahan C, Wang ZY, Dendukuri N, Lawn SD, Denkinger CM, et al. Lateral flow urine lipoarabinomannan assay for detecting active tuberculosis in HIV-positive adults. Cochrane Database of Systematic Reviews 2016, Issue 5. Art. No: CD011420. [DOI: 10.1002/14651858.CD011420.pub2]

\section{Shapiro 2020}

Shapiro AE, Ross JM, Schiller I, Kohli M, Dendukuri N, Steingart KR, et al. Xpert MTB/RIF and Xpert Ultra assays for pulmonary tuberculosis and rifampicin resistance in adults irrespective of signs or symptoms of pulmonary tuberculosis. Cochrane Database of Systematic Reviews 2020, Issue 7. Art. No: CD013694. [DOI: 10.1002/14651858.CD013694]

\section{Sharma 2004}

Sharma SK, Mohan A. Extrapulmonary tuberculosis. Indian Journal of Medical Research 2004;120(4):316-53.

\section{Sharma 2017b}

Sharma SK, Ryan H, Khaparde S, Sachdeva KS, Singh AD, Mohan A, et al. Index-TB guidelines: guidelines on extrapulmonary tuberculosis for India. Indian Journal of Medical Research 2017;145(4):448-63.

\section{Shen 2019}

Shen Y, Yu G, Zhong F, Kong X. Diagnostic accuracy of the Xpert MTB/RIF assay for bone and joint tuberculosis: a meta-analysis. PLOS One 2019;14(8):e0221427.

\section{Shu 2011}

Shu CC, Wang JT, Wang JY, Lee LN, Yu CJ. In-hospital outcome of patients with culture-confirmed tuberculous pleurisy: clinical 
impact of pulmonary involvement. BMC Infectious Diseases 2011;11:46. [DOI: 10.1186/1471-2334-11-46]

\section{Small 2011}

Small PM, Pai M. Tuberculosis diagnosis - time for a game change. New England Journal of Medicine 2011;363(111):1070-

\section{Stata 2017 [Computer program]}

Stata Statistical Software: Release 15. StataCorp. College Station, TX: StataCorp LP, 2017.

\section{Steingart 2014}

Steingart KR, Schiller I, Horne DJ, Pai M, Boehme CC, Dendukuri N. Xpert ${ }^{\circledR}$ MTB/RIF assay for pulmonary tuberculosis and rifampicin resistance in adults. Cochrane Database of Systematic Reviews 2014, Issue 1. Art. No: CD009593. [DOI: 10.1002/14651858.CD009593.pub3]

\section{Takwoingi 2013}

Takwoingi Y, Leeflang MM, Deeks JJ. Empirical evidence of the importance of comparative studies of diagnostic test accuracy. Annals of Internal Medicine 2013;158(7):544-54.

\section{Telenti 1993}

Telenti A, Imboden P, Marchesi F, Lowrie D, Cole S, Colston MJ, et al. Detection of rifampicin-resistance mutations in Mycobacterium tuberculosis. Lancet 1993;341(8846):647-50.

\section{Theron 2014a}

Theron G, Zijenah L, Chanda D, Clowes P, Rachow A, Lesosky M, et al. Feasibility, accuracy, and clinical effect of point-of-care Xpert MTB/RIF testing for tuberculosis in primary-care settings in Africa: a multicentre, randomised, controlled trial. Lancet 2014;383(9915):424-35.

\section{Thwaites 2013}

Thwaites GE, Van Toorn R, Schoeman J. Tuberculous meningitis: more questions, still too few answers. Lancet Neurology 2013;12(10):999-1010.

\section{Unitaid 2017}

Boyle D. Tuberculosis Diagnostics Technology and Market Landscape. 5th edition. Vernier: World Health Organization Unitaid Secretariat, 2017.

\section{Van Smeden 2014}

van Smeden M, Naaktgeboren CA, Reitsma JB, Moons KG, De Groot JA. Latent class models in diagnostic studies when there is no reference standard - a systematic review. American Journal of Epidemiology 2014;179(4):423-31.

\section{Vonasek 2020}

Vonasek B, Ness T, Takwoingi Y, Kay AW, Wyk SS, Ouellette L, et al. Screening tests for active pulmonary tuberculosis in children. Cochrane Database of Systematic Reviews 2020, Issue 7. Art. No: CD013693. [DOI: 10.1002/14651858.CD013693]

\section{Walzl 2018}

Walzl G, McNerney R, Du Plessis N, Bates M, McHugh TD, Chegou NN, et al. Tuberculosis: advances and challenges in development of new diagnostics and biomarkers. Lancet Infectious Diseases 2018;18(7):e199-e210.

\section{Webster 2014}

Webster AS, Shandera WX. The extrapulmonary dissemination of tuberculosis: a meta-analysis. International Journal of Mycobacteriology 2014;3:9-16.

\section{Whiting 2011}

Whiting PF, Rutjes AW, Westwood ME, Mallett S, Deeks JJ, Reitsma JB, et al. QUADAS-2: a revised tool for the quality assessment of diagnostic accuracy studies. Annals of Internal Medicine 2011;155(8):529-36.

\section{WHO 2010}

World Health Organization. Guidelines for treatment of tuberculosis, fourth edition. who.int/tb/ publications/2010/9789241547833/en/ 2010 (accessed 2 July 2020).

\section{WHO 2011}

World Health Organization. Rapid implementation of the Xpert MTB/RIF diagnostic test. Technical and operational 'Howto'. Practical considerations. who.int/tb/publications/tbamplificationtechnology-implementation/en/ 2011 (accessed 2 July 2020).

\section{WHO 2012}

World Health Organization. Updated interim critical concentrations for first-line and second-line DST (as of May 2012). www.stoptb.org/wg/gli/assets/documents/Updated $\% 20$ critical\%20concentration\%20table_1st\%20and\%202nd \%20line\%20drugs.pdf 2012 (accessed 2 July 2020).

\section{WHO 2014}

World Health Organization. Xpert MTB/RIF implementation manual. Technical and operational 'how-to' practical considerations. apps.who.int/iris/ bitstream/10665/112469/1/9789241506700_eng.pdf 2014 (accessed 2 July 2020).

\section{WHO 2017}

World Health Organization. Guidelines for the treatment of drug-susceptible tuberculosis and patient care (2017 update). www.who.int/tb/publications/2017/dstb_guidance_2017/en/ 2017 (accessed 1 April 2020).

\section{WHO 2018}

World Health Organization. Technical report on critical concentrations for drug susceptibility testing of medicines used in the treatment of drug-resistant tuberculosis (WHO/CDS/TB/2018.5). who.int/tb/publications/2018/ WHO_technical_report_concentrations_TB_drug_susceptibility/ en/ (accessed 2 July 2020).

\section{WHO Compendium 2018}

World Health Organization. Compendium of WHO guidelines and associated standards: ensuring optimum delivery of the cascade of care for patients with tuberculosis, second edition. www.who.int/tb/publications/ 
Compendium_WHO_guidelines_TB_2017/en/ (accessed 2 July 2020).

\section{WHO Consolidated Guidelines (Module 3) 2020}

World Health Organization. WHO consolidated guidelines on tuberculosis. Module 3: diagnosis - rapid diagnostics for tuberculosis detection. Licence: CC BY-NC-SA 3.0 IGO. who.int/publications/i/item/who-consolidated-guidelineson-tuberculosis-module-3-diagnosis---rapid-diagnostics-fortuberculosis-detection 2020 (accessed 2 July 2020).

\section{WHO Consolidated Guidelines (Module 4) 2020}

World Health Organization. WHO consolidated guidelines on tuberculosis. Module 4: treatment - drug-resistant tuberculosis treatment. Geneva: World Health Organization; 2020. Licence: CC BY-NC-SA 3.0 IGO. who.int/publications/i/ item/9789240007048 (accessed 2 July 2020).

\section{WHO END TB 2014}

World Health Organization. The END TB strategy. www.who.int/ tb/strategy/end-tb/en/ 2014 (accessed 1 April 2020).

\section{WHO Global TB Report 2020}

World Health Organization. Global Tuberculosis Report 2020. Licence: CC BY-NC-SA 3.0 IGO. Geneva: World Health Organization, 2020.

\section{WHO Operational Handbook Diagnosis (Module 3) 2020} World Health Organization. WHO operational handbook on tuberculosis. Module 3: diagnosis - rapid diagnostics for tuberculosis detection. Licence: CC BY-NC-SA 3.0 IGO. who.int/publications/i/item/who-operational-handbook-ontuberculosis-module-3-diagnosis---rapid-diagnostics-fortuberculosis-detection (accessed 6 August 2020).

\section{WHO Xpert MTB/RIF Policy 2011}

World Health Organization. Policy statement: automated real-time nucleic acid amplification technology for rapid and simultaneous detection of tuberculosis and rifampicin resistance: Xpert MTB/RIF system. WHO/HTM/TB/2011.4. www.who.int/tb/publications/tb-amplificationtechnologystatement/en/ 2011 (accessed 2 July 2020).

\section{WHO Xpert MTB/RIF Policy Update 2013}

World Health Organization. Automated real-time nucleic acid amplification technology for rapid and simultaneous detection of tuberculosis and rifampicin resistance: Xpert MTB/RIF system for the diagnosis of pulmonary and extrapulmonary TB in adults and children: policy update. WHO/HTM/TB/2013.14. apps.who.int/iris/handle/10665/112472 2013 (accessed 2 July 2020).

\section{WHO Xpert Ultra 2017}

World Health Organization. WHO meeting report of a technical expert consultation: non-inferiority analysis of Xpert MTB/ RIF Ultra compared to Xpert MTB/RIF. WHO/HTM/TB/2017.04. www.who.int/tb/publications/2017/XpertUltra/en/ 2017 (accessed 2 July 2020).

\section{Woodard 1982}

Woodard BH, Rosenberg SI, Farnham R, Adams DO. Incidence and nature of primary granulomatous inflammation in surgically removed material. American Journal of Surgical Pathology 1982;6(2):119-29.

\section{Woods 2001}

Woods GL. Molecular techniques in mycobacterial detection. In: Archives of Pathology \& Laboratory Medicine. Vol. 125. Geneva: World Health Organization, 2001:122-6.

\section{World Bank 2020}

World Bank. World bank list of economies. datahelpdesk.worldbank.org/knowledgebase/articles/906519world-bank-country-and-lending-groups 2019 (accessed 2 July 2020).

\section{Wright 2009a}

Wright CA, Bezuidenhout J. Histopathology and cytopathology. In: Schaaf HS, Zumla A, editors(s). Tuberculosis: A Comprehensive Clinical Reference. 1st edition. Amsterdam: Elsevier Science Publishers, 2009:205-15.

\section{Wright 2009b}

Wright CA, Hesseling AC, Bamford C, Burgess SM, Warren R, Marais BJ. Fine-needle aspiration biopsy: a first-line diagnostic procedure in paediatric tuberculosis suspects with peripheral lymphadenopathy? International Journal of Tuberculosis and Lung Disease 2009;13(11):1373-9.

\section{Yu 2019}

Yu G, Zhong F, Ye B, Xu X, Chen D, Shen Y. Diagnostic accuracy of the Xpert MTB/RIF assay for lymph node tuberculosis: a systematic review and meta-analysis. BioMed Research International 2019;2019:4878240.

\section{Zhang 2020}

Zhang M, Xue M, He JQ. Diagnostic accuracy of the new Xpert MTB/RIF Ultra for tuberculosis disease: a preliminary systematic review and meta-analysis. International Journal of Infectious Diseases 2020;90:35-45.

\section{References to other published versions of this review \\ Kohli 2017}

Kohli M, Schiller I, Dendukuri N, Ryan H, Dheda K, Denkinger CM, Schumacher SG, Steingart KR. Xpert ${ }^{\circledR}$ MTB/RIF assay for extrapulmonary tuberculosis and rifampicin resistance. Cochrane Database of Systematic Reviews 2017, Issue 8. Art. No: CD012768. [DOI: 10.1002/14651858.CD012768]

\section{Kohli 2018}

Kohli M, Schiller I, Dendukuri N, Dheda K, Denkinger CM, Schumacher SG, et al. Xpert ${ }^{\circledR}$ MTB/RIF assay for extrapulmonary tuberculosis and rifampicin resistance. Cochrane Database of Systematic Reviews 2018, Issue 8. Art. No: CD012768. [DOI: 10.1002/14651858.CD012768.pub2] 
CHARACTERISTICS OF STUDIES

Characteristics of included studies [ordered by study ID]

Ablanedo-Terrazas 2014

\section{Study characteristics}

Patient Sampling

Cross-sectional, consecutive, prospective

Patient characteristics and setting

Presenting signs and symptoms: HIV-positive patients with palpable cervical lymph nodes

Age: median 29 years [interquartile range (IQR) 24 to

36]

Sex, female: $12 \%$

Children: no

HIV infection: $100 \%$

Clinical setting: tertiary care centre (inpatient and outpatient)

Past history of TB: not reported

Patients on anti-TB treatment: no

Number of specimens evaluated: 15

Laboratory level: central

Country: Mexico

World Bank Income Classification: middle income

High TB burden: no

High TB/HIV burden: no

High MDR-TB burden: no

Xpert MTB/RIF

WHO standard operating procedure (SOP) or manufacturer's protocol followed: yes

Manufacturer's involvement: no

Target condition: lymph node (LN) TB

Reference standard for TB detection: Löwenstein-Jensen (LJ) and Mycobacterium growth indicator tube (MGIT)

Reference standard for rifampicin resistance: not reported

Speciation: yes

Decontamination: yes, $\mathrm{N}$-acetyl-L-cysteine-sodium hydroxide (NALC-NaOH) 
Ablanedo-Terrazas 2014 (Continued)

Comparative

Notes

Methodological quality

\begin{tabular}{lll}
\hline Item & $\begin{array}{l}\text { Authors' judge- } \\
\text { ment }\end{array}$ & $\begin{array}{l}\text { Risk of bias } \\
\text { Applicability } \\
\text { concerns }\end{array}$ \\
\hline
\end{tabular}

DOMAIN 1: Patient Selection

\begin{tabular}{ll}
\hline Was a consecutive or random sample of patients enrolled? & Yes \\
\hline Was a case-control design avoided? & Yes \\
\hline Did the study avoid inappropriate exclusions? & Yes \\
\hline Could the selection of patients have introduced bias?
\end{tabular}

Are there concerns that the included patients and setting do not Unclear match the review question?

\section{DOMAIN 2: Index Test (Xpert MTB/RIF)}

Were the index test results interpreted without knowledge of the results of Yes the reference standard?

If a threshold was used, was it pre-specified? Yes

Could the conduct or interpretation of the index test have introduced Low risk
bias?
bias?

Are there concerns that the index test, its conduct, or interpretation differ from the review question?

\section{DOMAIN 2: Index Test (Xpert Ultra)}

DOMAIN 3: Reference Standard

Is the reference standards likely to correctly classify the target condition? Unclear

Were the reference standard results interpreted without knowledge of the Unclear results of the index tests?

For rifampicin resistance testing, were the reference standard results in- Unclear terpreted without knowledge of the results of the index test?

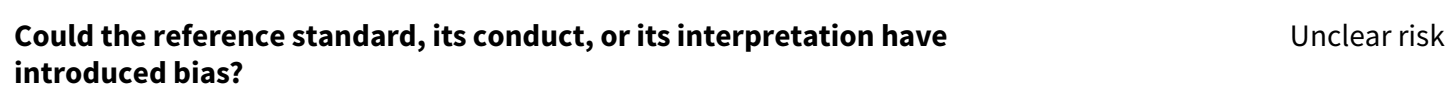

\section{Are there concerns that the target condition as defined by the refer-} ence standard does not match the question?

\section{DOMAIN 4: Flow and Timing}

Was there an appropriate interval between index test and reference stan- $\quad$ Yes
dard? dard? 
Ablanedo-Terrazas 2014 (Continued)

Did all patients receive the same reference standard?

Yes

Were all patients included in the analysis?

Yes

Could the patient flow have introduced bias?

Low risk

Ajbani 2018

\section{Study characteristics}

\section{Patient Sampling}

Patient characteristics and setting
Cross-sectional, consecutive, prospective

Presenting signs and symptoms: neck rigidity, vomiting, fever, headache and seizures

Age: 13 and older

Sex, female: $46 \%$

Children: no

HIV infection: not reported

Clinical setting: tertiary care centre (inpatient)

Past history of TB: not reported

Participants on anti-TB treatment: not reported

Number of specimens evaluated: 13

Laboratory level: central

Country: India

World Bank Income Classification: middle income

High TB burden: yes

High TB/HIV burden: yes

High MDR-TB burden: yes

Index tests

Xpert MTB/RIF

WHO SOP or manufacturer's protocol followed: not reported

Decontamination: no

\footnotetext{
Target condition and reference standard(s)
}

TB meningitis

MGIT

Speciation: yes

Flow and timing

Comparative 
Ajbani 2018 (Continued)

Notes

\section{Methodological quality}

\begin{tabular}{|c|c|c|c|}
\hline Item & $\begin{array}{l}\text { Authors' } \\
\text { judgement }\end{array}$ & Risk of bias & $\begin{array}{l}\text { Applicability } \\
\text { concerns }\end{array}$ \\
\hline
\end{tabular}

\section{DOMAIN 1: Patient Selection}

Was a consecutive or random sample of patients enrolled? Yes

Was a case-control design avoided? Yes

Did the study avoid inappropriate exclusions? Yes

Could the selection of patients have introduced bias? Low risk

Are there concerns that the included patients and setting do not match the review question?

\section{DOMAIN 2: Index Test (Xpert MTB/RIF)}

Were the index test results interpreted without knowledge of the results of Yes the reference standard?

If a threshold was used, was it pre-specified?

Could the conduct or interpretation of the index test have introduced L L risk
bias?
bias?

Are there concerns that the index test, its conduct, or interpretation differ from the review question?

\section{DOMAIN 2: Index Test (Xpert Ultra)}

\section{DOMAIN 3: Reference Standard}

\begin{tabular}{|c|}
\hline Is the reference standards likely to correctly classify the target condition? \\
\hline
\end{tabular}

For rifampicin resistance testing, were the reference standard results interpreted without knowledge of the results of the index test?

Could the reference standard, its conduct, or its interpretation have in- Low risk
troduced bias?

\section{Are there concerns that the target condition as defined by the reference standard does not match the question?}

\section{DOMAIN 4: Flow and Timing}

Was there an appropriate interval between index test and reference stan- Yes dard?

Did all patients receive the same reference standard?

Yes 
Ajbani 2018 (Continued)

Were all patients included in the analysis?

Yes

Could the patient flow have introduced bias?

Low risk

\section{Al-Ateah 2012}

\section{Study characteristics}

\begin{tabular}{|c|c|}
\hline Patient Sampling & Cross-sectional, consecutive, prospective \\
\hline \multirow[t]{15}{*}{ Patient characteristics and setting } & $\begin{array}{l}\text { Presenting signs and symptoms: patients suspected } \\
\text { of having extrapulmonary TB }\end{array}$ \\
\hline & Age: median 35 years \\
\hline & Sex, female: $45 \%$ \\
\hline & Children: $3 \%$ \\
\hline & HIV infection: $0 \%$ \\
\hline & $\begin{array}{l}\text { Clinical setting: tertiary care centre (laboratory-based } \\
\text { evaluation) }\end{array}$ \\
\hline & Past history of TB: not reported \\
\hline & Patients on anti-TB treatment: not reported \\
\hline & Number of specimens evaluated: 67 \\
\hline & Laboratory level: central \\
\hline & Country: Saudi Arabia \\
\hline & World Bank Income Classification: high income \\
\hline & High TB burden: no \\
\hline & High TB/HIV burden: no \\
\hline & High MDR-TB burden: no \\
\hline \multirow[t]{3}{*}{ Index tests } & Xpert MTB/RIF \\
\hline & WHO SOP or manufacturer's protocol followed: yes \\
\hline & Manufacturer's involvement: no \\
\hline \multirow[t]{4}{*}{ Target condition and reference standard(s) } & $\begin{array}{l}\text { Target condition: lymph node TB, pleural TB } \\
\text { Reference standards for TB detection: LJ and MGIT }\end{array}$ \\
\hline & $\begin{array}{l}\text { Reference standard for rifampicin resistance: MGIT- } \\
\text { drug susceptibility testing (DST) }\end{array}$ \\
\hline & Speciation: yes \\
\hline & Decontamination: yes, NALC-NaOH \\
\hline
\end{tabular}

Flow and timing 
Al-Ateah 2012 (Continued)

Comparative

Notes

Site of extrapulmonary disease was not reported for

16 tissue specimens and 10 abscesses

\section{Methodological quality}

\begin{tabular}{|c|c|c|c|}
\hline Item & $\begin{array}{l}\text { Authors' } \\
\text { judgement }\end{array}$ & Risk of bias & $\begin{array}{l}\text { Applicability } \\
\text { concerns }\end{array}$ \\
\hline
\end{tabular}

\section{DOMAIN 1: Patient Selection}

Was a consecutive or random sample of patients enrolled? Yes

Was a case-control design avoided? Yes

Did the study avoid inappropriate exclusions? Yes

Could the selection of patients have introduced bias? Low risk

Are there concerns that the included patients and setting do not match Unclear
the review question?

DOMAIN 2: Index Test (Xpert MTB/RIF)

Were the index test results interpreted without knowledge of the results of Yes the reference standard?

If a threshold was used, was it pre-specified? Yes

Could the conduct or interpretation of the index test have introduced Low risk
bias?

bias?

Are there concerns that the index test, its conduct, or interpretation dif-

fer from the review question?

DOMAIN 2: Index Test (Xpert Ultra)

\section{DOMAIN 3: Reference Standard}

Is the reference standards likely to correctly classify the target condition? Unclear

Were the reference standard results interpreted without knowledge of the Yes results of the index tests?

For rifampicin resistance testing, were the reference standard results inter- Yes preted without knowledge of the results of the index test?

Could the reference standard, its conduct, or its interpretation have in- Unclear risk
troduced bias?
troduced bias?

Are there concerns that the target condition as defined by the reference standard does not match the question?

\section{DOMAIN 4: Flow and Timing}


Al-Ateah 2012 (Continued)

Was there an appropriate interval between index test and reference stan- Yes dard?

Did all patients receive the same reference standard?

Yes

Were all patients included in the analysis?

Yes

\section{Could the patient flow have introduced bias?}

Low risk

Antel 2020

\section{Study characteristics}

\begin{tabular}{|c|c|}
\hline Patient Sampling & Cross-sectional, consecutive, prospective \\
\hline \multirow[t]{15}{*}{ Patient characteristics and setting } & $\begin{array}{l}\text { Presenting signs and symptoms: suspected tuberculosis } \\
\text { adenitis }\end{array}$ \\
\hline & Age: $\geq 18$ years; median 37 years (IQR 30 to 49 ) \\
\hline & Sex, female: $55 \%$ \\
\hline & Children: no \\
\hline & HIV infection: $51 \%$ \\
\hline & $\begin{array}{l}\text { Clinical setting: tertiary referral centre, inpatients and } \\
\text { outpatients, most participants ( } 84 \%) \text { were seen as outpa- } \\
\text { tients, high percentage received prior testing for tubercu- } \\
\text { losis, see note }\end{array}$ \\
\hline & Past history of TB: $24 \%$ \\
\hline & Patients on anti-TB treatment: $21 \%$ \\
\hline & Number of specimens evaluated: 99 \\
\hline & Laboratory level: central \\
\hline & Country: South Africa \\
\hline & World Bank Income Classification: middle income \\
\hline & High TB burden: yes \\
\hline & High TB/HIV burden: yes \\
\hline & High MDR-TB burden: yes \\
\hline Index tests & Xpert Ultra \\
\hline \multirow[t]{5}{*}{ Target condition and reference standard(s) } & $\begin{array}{l}\text { Target condition: lymph node tuberculosis, specimen col- } \\
\text { lected by fine-needle biopsy and core-needle biopsy }\end{array}$ \\
\hline & Reference standard: MGIT \\
\hline & Target condition: rifampicin resistance \\
\hline & Reference standard: MTBDRplus \\
\hline & Speciation: yes, MTBDRplus \\
\hline
\end{tabular}


Antel 2020 (Continued)

Decontamination: no

Flow and timing

Comparative

Notes

"A high proportion of participants had tuberculosis investigations prior to referral and the frequency of positive results were: sputum Xpert 3/22, urinary LAM $1 / 5$, and tuberculosis culture $(5 / 15)$ (by site: urine $0 / 1$, blood $1 / 2$, sputum $4 / 12$, lymph node $0 / 1$ (tissue)). Chest $x$-ray had been performed in $36 \%$ and reported as 'suggestive of tuberculosis' by the referring clinician in $28 \%$ of these."

\section{Methodological quality}

\begin{tabular}{lll}
\hline Item & $\begin{array}{l}\text { Authors' judge- } \\
\text { ment }\end{array}$ & Risk of bias $\begin{array}{l}\text { Applicability } \\
\text { concerns }\end{array}$ \\
\hline
\end{tabular}

\section{DOMAIN 1: Patient Selection}

\begin{tabular}{lc}
\hline Was a consecutive or random sample of patients enrolled? & Yes \\
\hline Was a case-control design avoided? & Yes \\
\hline Did the study avoid inappropriate exclusions? & Yes
\end{tabular}

Could the selection of patients have introduced bias?

Low risk

Are there concerns that the included patients and setting do not

Unclear match the review question?

\section{DOMAIN 2: Index Test (Xpert MTB/RIF)}

Were the index test results interpreted without knowledge of the results Yes of the reference standard?

If a threshold was used, was it pre-specified? Yes

Could the conduct or interpretation of the index test have intro- Low risk
duced bias?

\section{duced bias?}

Are there concerns that the index test, its conduct, or interpretation differ from the review question?

\section{DOMAIN 2: Index Test (Xpert Ultra)}

Were the index test results interpreted without knowledge of the results Yes

of the reference standard?
If a threshold was used, was it pre-specified?
Yes
Could the conduct or interpretation of the index test have intro- duced bias?
Low risk

Are there concerns that the index test, its conduct, or interpretation differ from the review question? 
Antel 2020 (Continued)

\section{DOMAIN 3: Reference Standard}

Is the reference standards likely to correctly classify the target condi- Yes tion?

Were the reference standard results interpreted without knowledge of Yes the results of the index tests?

For rifampicin resistance testing, were the reference standard results Yes interpreted without knowledge of the results of the index test?

Could the reference standard, its conduct, or its interpretation have Low risk
introduced bias?

Are there concerns that the target condition as defined by the refer-

Low concern ence standard does not match the question?

\section{DOMAIN 4: Flow and Timing}

Was there an appropriate interval between index test and reference Yes standard?

\begin{tabular}{lcl}
\hline Did all patients receive the same reference standard? & Yes \\
\hline Were all patients included in the analysis? & Yes & Low risk \\
\hline Could the patient flow have introduced bias? & \\
\hline
\end{tabular}

Azevedo 2018

\section{Study characteristics}

Patient Sampling

Cross-sectional, manner of participant selection not reported, retrospective

Patient characteristics and setting

Presenting signs and symptoms: patients with suspected meningitis

Age: > 16 years

Sex, female: not reported

Children: no

HIV infection: $100 \%$

Clinical setting: tertiary care centre (inpatient)

Past history of TB: not reported

Participants on anti-TB treatment: not reported

Number of specimens evaluated: 101

Laboratory level: central, university medical centre

Country: Brazil 
High TB burden: yes

High TB/HIV burden: yes

High MDR-TB burden: no

WHO SOP or manufacturer's protocol followed: not reported

\title{
Target condition and reference standard(s)
}

\author{
TB meningitis \\ Culture not otherwise specified; CRS: uniform case de- \\ finition \\ Speciation: not reported \\ Decontamination: no
}

\begin{abstract}
Flow and timing
\end{abstract}
Comparative

$$
\text { Notes }
$$

\section{Methodological quality}

\begin{tabular}{lll}
\hline Item & $\begin{array}{l}\text { Authors' } \\
\text { judgement }\end{array}$ & $\begin{array}{c}\text { Risk of bias } \\
\text { Concerns }\end{array}$ \\
\hline DOMAIN 1: Patient Selection & Unclear & Yes \\
\hline Was a consecutive or random sample of patients enrolled? & Yes & Unclear risk \\
\hline Was a case-control design avoided? & & \\
\hline Did the study avoid inappropriate exclusions? & & Low concern \\
\hline Could the selection of patients have introduced bias? & \\
\hline $\begin{array}{l}\text { Are there concerns that the included patients and setting do not match } \\
\text { the review question? }\end{array}$ & \\
\hline
\end{tabular}

\section{DOMAIN 2: Index Test (Xpert MTB/RIF)}

Were the index test results interpreted without knowledge of the results of Yes the reference standard?

If a threshold was used, was it pre-specified? Yes

\section{Could the conduct or interpretation of the index test have introduced bias?}

Are there concerns that the index test, its conduct, or interpretation dif-

fer from the review question? 
Azevedo 2018 (Continued)

DOMAIN 2: Index Test (Xpert Ultra)

\section{DOMAIN 3: Reference Standard}

Is the reference standards likely to correctly classify the target condition? Yes

Were the reference standard results interpreted without knowledge of the Unclear results of the index tests?

For rifampicin resistance testing, were the reference standard results inter-

preted without knowledge of the results of the index test?

\section{Could the reference standard, its conduct, or its interpretation have in- troduced bias? \\ Unclear risk}

Are there concerns that the target condition as defined by the reference

Unclear standard does not match the question?

\section{DOMAIN 4: Flow and Timing}

Was there an appropriate interval between index test and reference stan- $\quad$ Yes dard?

Did all patients receive the same reference standard? Yes

Were all patients included in the analysis?

Yes

\section{Study characteristics}

Patient Sampling Cross-sectional, consecutive, prospective

Patient characteristics and setting

Presenting signs and symptoms: HIV-infected patients presenting with symptoms of meningitis being evaluated for cryptococcal meningitis. All persons who were CSF cryptococcal antigen-negative had a TB workup

Age: median 40 years (IQR 30 to 45 )

Sex, female: $34 \%$

Children: no

HIV infection: $98 \%$

Clinical setting: tertiary care centre (Inpatient)

Past history of TB: $22 \%$

Participants on anti-TB treatment: yes, $11 \%$

Number of specimens evaluated: 80

Laboratory level: central 
Bahr 2015 (Continued)

Country: Uganda

World Bank Income Classification: low income

High TB burden: no

High TB/HIV burden: yes

High MDR-TB burden: no

Index tests

Xpert MTB/RIF

WHO SOP or manufacturer's protocol followed: yes

Manufacturer's involvement: no

Target condition and reference standard(s)

Target condition: TB meningitis

Reference standard for TB detection: LJ and MGIT

Reference standard for rifampicin resistance: MGIT-DST

Speciation: yes

Decontamination: no

Flow and timing

\section{Comparative}

Notes

Reference standards were culture and a TB meningitis

uniform case definition

\section{Methodological quality}

\begin{tabular}{lll}
\hline Item & $\begin{array}{l}\text { Authors' judge- } \\
\text { ment }\end{array}$ & $\begin{array}{l}\text { Risk of bias } \\
\text { Applicability } \\
\text { concerns }\end{array}$ \\
\hline
\end{tabular}

DOMAIN 1: Patient Selection

\begin{tabular}{lcl}
\hline Was a consecutive or random sample of patients enrolled? & Yes & Yes \\
\hline Was a case-control design avoided? & Yes & Low risk \\
\hline Did the study avoid inappropriate exclusions? & \\
\hline Could the selection of patients have introduced bias? & Low concern \\
\hline $\begin{array}{l}\text { Are there concerns that the included patients and setting do not } \\
\text { match the review question? }\end{array}$
\end{tabular}

\section{DOMAIN 2: Index Test (Xpert MTB/RIF)}

Were the index test results interpreted without knowledge of the results Yes of the reference standard?

If a threshold was used, was it pre-specified? Yes

\section{Could the conduct or interpretation of the index test have introduced bias?}

Low risk 
Bahr 2015 (Continued)

Are there concerns that the index test, its conduct, or interpretation differ from the review question?

\section{DOMAIN 2: Index Test (Xpert Ultra)}

\section{DOMAIN 3: Reference Standard}

Is the reference standards likely to correctly classify the target condition? Yes

Were the reference standard results interpreted without knowledge of Yes the results of the index tests?

For rifampicin resistance testing, were the reference standard results in- Yes terpreted without knowledge of the results of the index test?

\begin{tabular}{l}
$\begin{array}{l}\text { Could the reference standard, its conduct, or its interpretation have } \\
\text { introduced bias? }\end{array}$ \\
\hline
\end{tabular}

Are there concerns that the target condition as defined by the refer-

Low concern ence standard does not match the question?

\section{DOMAIN 4: Flow and Timing}

Was there an appropriate interval between index test and reference stan- Yes dard?

\begin{tabular}{lcl}
\hline Did all patients receive the same reference standard? & Yes \\
\hline Were all patients included in the analysis? & Yes & Low risk \\
\hline Could the patient flow have introduced bias? & \\
\hline
\end{tabular}

Bahr 2017

\section{Study characteristics}

Presenting signs and symptoms: HIV-infected patients presenting with symptoms of meningitis being evaluated for cryptococcal meningitis. All persons who were CSF cryptococcal antigen-negative had a TB workup

Age: TB meningitis: median 32 years (IQR 30 to 34); other meningitis: 34 years (IQR 29 to 43 )

Sex, female: $45 \%$

Children: no

HIV infection: $100 \%$

Clinical setting: tertiary care centre (inpatient)

Past history of TB: $6 \%$ 
Bahr 2017 (Continued)

\author{
Participants on anti-TB treatment: yes, 2\% \\ Number of specimens evaluated: 129 \\ Laboratory level: central \\ Country: Uganda \\ World Bank Income Classification: low income \\ High TB burden: no \\ High TB/HIV burden: yes \\ High MDR-TB burden: no
}

Index tests

Xpert MTB/RIF and Xpert Ultra

WHO SOP or manufacturer's protocol followed: yes

Manufacturer's involvement: no

\title{
Target condition and reference standard(s)
}
Target condition: TB meningitis
Reference standard for TB detection: MGIT
Reference standard for rifampicin resistance: MGIT- DST
Speciation: yes
Decontamination: no

\section{Flow and timing}

\section{Comparative}

\section{Notes}

This study evaluated Xpert MTB/RIF and Xpert MTB/ RIF Ultra.

Reference standards were culture and a TB meningitis uniform case definition

\section{Methodological quality}

\begin{tabular}{lll}
\hline Item & $\begin{array}{l}\text { Authors' } \\
\text { judgement }\end{array}$ & $\begin{array}{c}\text { Risk of bias } \\
\text { Applicability } \\
\text { concerns }\end{array}$ \\
\hline
\end{tabular}

DOMAIN 1: Patient Selection

Was a consecutive or random sample of patients enrolled?

Was a case-control design avoided?

Did the study avoid inappropriate exclusions?

Could the selection of patients have introduced bias?

Are there concerns that the included patients and setting do not match the review question?
Yes

Yes

Yes 
Bahr 2017 (Continued)

DOMAIN 2: Index Test (Xpert MTB/RIF)

Were the index test results interpreted without knowledge of the results of Yes the reference standard?

If a threshold was used, was it pre-specified?

Yes

\section{Could the conduct or interpretation of the index test have introduced} bias?

\section{Are there concerns that the index test, its conduct, or interpretation dif-}

fer from the review question?

\section{DOMAIN 2: Index Test (Xpert Ultra)}

Were the index test results interpreted without knowledge of the results of Yes

the reference standard?

If a threshold was used, was it pre-specified?

Yes

Could the conduct or interpretation of the index test have introduced Low risk
bias?

bias?

Are there concerns that the index test, its conduct, or interpretation dif-

fer from the review question?

\section{DOMAIN 3: Reference Standard}

Is the reference standards likely to correctly classify the target condition? Yes

Were the reference standard results interpreted without knowledge of the Yes results of the index tests?

For rifampicin resistance testing, were the reference standard results inter- Yes preted without knowledge of the results of the index test?

\section{Could the reference standard, its conduct, or its interpretation have in- Low risk troduced bias?}

\section{Are there concerns that the target condition as defined by the reference} standard does not match the question?

\section{DOMAIN 4: Flow and Timing}

Was there an appropriate interval between index test and reference stan- Yes dard?

Did all patients receive the same reference standard?

Yes

Were all patients included in the analysis?

Yes

Could the patient flow have introduced bias?

Low risk 
Bera 2015

\section{Study characteristics}

\section{Patient Sampling}

Patient characteristics and setting
Cross-sectional, consecutive, prospective

Presenting signs and symptoms: patients with exudative ascites (lymphocytic ascites and ascitic fluid protein content $>2.5 \mathrm{~g} / \mathrm{dL}$ )

Age: mean 43 years (standard deviation (SD) 15 years)

Sex, female: $29 \%$

Children: no

HIV infection: not reported

Clinical setting: tertiary care centre (outpatient)

Past history of TB: not reported

Patients on anti-TB treatment: not reported

Number of specimens evaluated: 28

Laboratory level: central

Country: India

World Bank Income Classification: middle income

High TB burden: yes

High TB/HIV burden: yes

High MDR-TB burden: yes

Index tests

Xpert MTB/RIF

WHO SOP or manufacturer's protocol followed: not reported

Manufacturer's involvement: no

Target condition and reference standard(s)
Target condition: peritoneal TB

Reference standard for TB detection: LJ and MGIT

Reference standard for rifampicin resistance: LJ and MGIT-DST

Speciation: not reported

Decontamination: no

Flow and timing

\section{Comparative}

Notes

"The study included only smear-negative specimens, however, the study excluded specimens that were negative for malignant cells on prior testing (i.e. cytology)"

\section{Methodological quality}

Xpert MTB/RIF Ultra and Xpert MTB/RIF assays for extrapulmonary tuberculosis and rifampicin resistance in adults (Review) 
Bera 2015 (Continued)

$\begin{array}{lll}\text { Item } & \begin{array}{l}\text { Authors'judge- } \\ \text { ment }\end{array} & \begin{array}{l}\text { Applisk of bias } \\ \text { concerns }\end{array}\end{array}$

DOMAIN 1: Patient Selection

Was a consecutive or random sample of patients enrolled? Yes

\begin{tabular}{ll}
\hline Was a case-control design avoided? & Yes
\end{tabular}

Did the study avoid inappropriate exclusions? No

Could the selection of patients have introduced bias? Unclear risk

Are there concerns that the included patients and setting do not
match the review question?

DOMAIN 2: Index Test (Xpert MTB/RIF)

Were the index test results interpreted without knowledge of the results Yes of the reference standard?

If a threshold was used, was it pre-specified? Yes Could the conduct or interpretation of the index test have intro- Low risk
duced bias?

Are there concerns that the index test, its conduct, or interpretation differ from the review question?

Unclear

DOMAIN 2: Index Test (Xpert Ultra)

\section{DOMAIN 3: Reference Standard}

Is the reference standards likely to correctly classify the target condi- Yes tion?

Were the reference standard results interpreted without knowledge of Yes the results of the index tests?

For rifampicin resistance testing, were the reference standard results Yes interpreted without knowledge of the results of the index test?

Could the reference standard, its conduct, or its interpretation have Low risk
introduced bias?

Are there concerns that the target condition as defined by the reference standard does not match the question?

\section{DOMAIN 4: Flow and Timing}

Was there an appropriate interval between index test and reference standard?

\begin{tabular}{ll}
\hline Did all patients receive the same reference standard? & Yes \\
\hline Were all patients included in the analysis? & Yes \\
\hline
\end{tabular}


Bera 2015 (Continued)

Biadglegne 2014

\section{Study characteristics}

\section{Patient Sampling}

Patient characteristics and setting
Cross-sectional, consecutive, prospective

Presenting signs and symptoms: patients with enlarged lymph nodes not responding to a 2-week course of antibiotics and clinically suspected for TB lymphadenitis

Age: $\leq 14$ years: $15 \%$; > 14 years: $85 \%$

Sex, female: $57 \%$

Children: $15 \%$

HIV infection: not reported

Clinical setting: tertiary care centres (multicentre study)

Past history of TB: not reported

Patients on anti-TB treatment: not reported

Number of specimens evaluated: 213

Laboratory level: intermediate

Country: Ethiopia

World Bank Income Classification: low income

High TB burden: yes

High TB/HIV burden: yes

High MDR-TB burden: yes
Xpert MTB/RIF

WHO SOP or manufacturer's protocol followed: yes

Manufacturer's involvement: no

Target condition: lymph node TB

Reference standard for TB detection: LJ and Gottsascker and BacT/ALERT 3D

Reference standard for rifampicin resistance: MTBDRplus and BacT-DST

Speciation: yes

Decontamination: yes, NALC-NaOH

Flow and timing

Comparative

Xpert MTB/RIF Ultra and Xpert MTB/RIF assays for extrapulmonary tuberculosis and rifampicin resistance in adults (Review) 
Biadglegne 2014 (Continued)

Notes

Total number of participants: 231 ; included: 213 (exclud-

ed: contaminated = 11 ; invalid /error $=7$ )

\section{Methodological quality}

\begin{tabular}{lll}
\hline Item & $\begin{array}{l}\text { Authors' judge- } \\
\text { ment }\end{array}$ & Risk of bias $\begin{array}{l}\text { Applicability } \\
\text { concerns }\end{array}$ \\
\hline
\end{tabular}

DOMAIN 1: Patient Selection

\begin{tabular}{lcc}
\hline Was a consecutive or random sample of patients enrolled? & Yes & Yes \\
\hline Was a case-control design avoided? & Low risk \\
\hline Did the study avoid inappropriate exclusions? & \\
\hline Could the selection of patients have introduced bias? & Unclear \\
\hline $\begin{array}{l}\text { Are there concerns that the included patients and setting do not } \\
\text { match the review question? }\end{array}$
\end{tabular}

\section{DOMAIN 2: Index Test (Xpert MTB/RIF)}

Were the index test results interpreted without knowledge of the results Yes of the reference standard?

If a threshold was used, was it pre-specified? Yes
$\begin{aligned} & \text { Could the conduct or interpretation of the index test have intro- } \\ & \text { duced bias? }\end{aligned}$

duced bias?

Are there concerns that the index test, its conduct, or interpretation differ from the review question?

DOMAIN 2: Index Test (Xpert Ultra)

\section{DOMAIN 3: Reference Standard}

Is the reference standards likely to correctly classify the target condi- Unclear tion?

Were the reference standard results interpreted without knowledge of Yes the results of the index tests?

For rifampicin resistance testing, were the reference standard results Yes interpreted without knowledge of the results of the index test?

\section{Could the reference standard, its conduct, or its interpretation have Unclear risk introduced bias?}

\section{Are there concerns that the target condition as defined by the refer- ence standard does not match the question?}

\section{DOMAIN 4: Flow and Timing}

Was there an appropriate interval between index test and reference standard? 
Biadglegne 2014 (Continued)

Did all patients receive the same reference standard?

Yes

Were all patients included in the analysis?

Yes

Could the patient flow have introduced bias?

Low risk

Blaich 2014

\section{Study characteristics}

\section{Patient Sampling}

Patient characteristics and setting
Cross-sectional, consecutive, prospective

Presenting signs and symptoms: patients with suspicion of extrapulmonary TB

Age: median 34 (IQR 30 to 52)

Sex, female: $46 \%$

Children: no

HIV infection: yes, $8 \%$

Clinical setting: university hospital (inpatient and outpatient)

Past history of TB: yes, $11 \%$

Patients on anti-TB treatment: no

Number of specimens evaluated: 20

Laboratory level: central

Country: Switzerland

World Bank Income Classification: high income

High TB burden: no

High TB/HIV burden: no

High MDR-TB burden: no
Xpert MTB/RIF

WHO SOP or manufacturer's protocol followed: yes for lymph node aspirate, bone and joint fluid, urine, peritoneal fluid, and lymph node tissue; no for CSF

Manufacturer's involvement: no

\footnotetext{
Target condition and reference standard(s)
}

\author{
Target condition: pleural TB, TB meningitis, lymph node \\ $T B$, pericardial TB, genitourinary TB, bone and joint TB \\ Reference standard for TB detection: LJ and MGIT \\ Reference standard for rifampicin resistance: MGIT-DST \\ Speciation: yes
}


Blaich 2014 (Continued)

Decontamination: yes, NALC-NaOH for all specimens except pleural fluid and CSF

Flow and timing

Comparative

\begin{tabular}{|c|c|c|c|}
\hline Notes & $\begin{array}{l}\text { Study included } 1 \\
\text { both aspirate an }\end{array}$ & $\begin{array}{l}\text { e marrow spe } \\
\text { sue }\end{array}$ & that consisted of \\
\hline \multicolumn{4}{|c|}{ Methodological quality } \\
\hline Item & $\begin{array}{l}\text { Authors' judge- } \\
\text { ment }\end{array}$ & Risk of bias & $\begin{array}{l}\text { Applicability } \\
\text { concerns }\end{array}$ \\
\hline
\end{tabular}

DOMAIN 1: Patient Selection

\begin{tabular}{lcc}
\hline Was a consecutive or random sample of patients enrolled? & Yes & Yes \\
\hline Was a case-control design avoided? & Yes & Low risk \\
\hline Did the study avoid inappropriate exclusions? & \\
\hline Could the selection of patients have introduced bias? & Unclear \\
\hline $\begin{array}{l}\text { Are there concerns that the included patients and setting do not } \\
\text { match the review question? }\end{array}$
\end{tabular}

\section{DOMAIN 2: Index Test (Xpert MTB/RIF)}

Were the index test results interpreted without knowledge of the re- Yes

sults of the reference standard?

If a threshold was used, was it pre-specified? Yes

Could the conduct or interpretation of the index test have intro- Low risk
duced bias?

Are there concerns that the index test, its conduct, or interpretation differ from the review question?

\section{DOMAIN 2: Index Test (Xpert Ultra)}

\section{DOMAIN 3: Reference Standard}

Is the reference standards likely to correctly classify the target condi- Unclear tion?

Were the reference standard results interpreted without knowledge of No the results of the index tests?

For rifampicin resistance testing, were the reference standard results Yes interpreted without knowledge of the results of the index test?

\section{Could the reference standard, its conduct, or its interpretation have introduced bias?


Blaich 2014 (Continued)

\section{Are there concerns that the target condition as defined by the ref- erence standard does not match the question?}

\section{DOMAIN 4: Flow and Timing}

Was there an appropriate interval between index test and reference Yes standard?

Did all patients receive the same reference standard? Yes

Were all patients included in the analysis?

Yes

Could the patient flow have introduced bias?

Low risk

Causse 2011

\section{Study characteristics}

Patient Sampling

Cross-sectional, consecutive, prospective

Patient characteristics and setting

Presenting signs and symptoms: not reported

Age: median 45 years, range 5 to 83 years

Sex, female: $31 \%$

Children: yes, $15 \%$

HIV infection: not reported

Clinical setting: tertiary care centre (inpatient)

Past history of TB: not reported

Patients on anti-TB treatment: not reported

Number of specimens evaluated: 261

Laboratory level: central

Country: Spain

World Bank Income Classification: high income

High TB burden: no

High TB/HIV burden: no

High MDR-TB burden: no

Index tests

Xpert MTB/RIF

WHO SOP or manufacturer's protocol followed: no

Manufacturer's involvement: no

Target condition and reference standard(s)

Target condition: pleural TB, lymph node TB, TB meningitis, peritoneal TB, pericardial TB, genitourinary TB 
Flow and timing

Comparative

Notes

Methodological quality

\begin{tabular}{|c|c|c|c|}
\hline Item & $\begin{array}{l}\text { Authors' } \\
\text { judgement }\end{array}$ & Risk of bias & $\begin{array}{l}\text { Applicability } \\
\text { concerns }\end{array}$ \\
\hline
\end{tabular}

DOMAIN 1: Patient Selection

\begin{tabular}{lcl}
\hline Was a consecutive or random sample of patients enrolled? & Yes & Yes \\
\hline Was a case-control design avoided? & Yes & Low risk \\
\hline Did the study avoid inappropriate exclusions? & \\
\hline Could the selection of patients have introduced bias? & Unclear \\
\hline $\begin{array}{l}\text { Are there concerns that the included patients and setting do not match } \\
\text { the review question? }\end{array}$
\end{tabular}

\section{DOMAIN 2: Index Test (Xpert MTB/RIF)}

Were the index test results interpreted without knowledge of the results of Yes the reference standard?

If a threshold was used, was it pre-specified?

Yes

Could the conduct or interpretation of the index test have introduced Low risk
bias?

Could the conduct or interpretation of the index test have introduced

Are there concerns that the index test, its conduct, or interpretation dif-

fer from the review question?

DOMAIN 2: Index Test (Xpert Ultra)

\section{DOMAIN 3: Reference Standard}

Is the reference standards likely to correctly classify the target condition? Unclear

Were the reference standard results interpreted without knowledge of the Yes results of the index tests?

For rifampicin resistance testing, were the reference standard results inter- Unclear preted without knowledge of the results of the index test? 
Causse 2011 (Continued)

Could the reference standard, its conduct, or its interpretation have introduced bias?

Are there concerns that the target condition as defined by the reference

Low concern standard does not match the question?

\section{DOMAIN 4: Flow and Timing}

Was there an appropriate interval between index test and reference stan- Yes dard?

Did all patients receive the same reference standard? Yes

Were all patients included in the analysis? Yes

Could the patient flow have introduced bias?

Low risk

Che 2017

\section{Study characteristics}

Patient Sampling

Cross-sectional, prospective, and consecutive

Patient characteristics and setting

Presenting signs and symptoms: patients with evidence of pleural effusion demonstrated by X-ray, suspected to have tuberculosis pleurisy

Age: median 44 years, range 18 to 83 years

Sex, female: $31 \%$

Children: no

HIV infection: $1 \%$

Clinical setting: tertiary care centre (inpatient)

Past history of TB: not reported

Patients on anti-TB treatment: not reported

Number of specimens evaluated: 78

Laboratory level: central

Country: China

World Bank Income Classification: middle income

High TB burden: yes

High TB/HIV burden: yes

High MDR-TB burden: yes 
Che 2017 (Continued)

Manufacturer's involvement: no

Target condition and reference standard(s)

Target condition: pleural TB

Reference standard for TB detection: MGIT

Reference standard for rifampicin resistance: not reported

Speciation: yes

Decontamination: no

Flow and timing

Comparative

Notes

Methodological quality

\begin{tabular}{|c|c|c|c|}
\hline Item & $\begin{array}{l}\text { Authors' } \\
\text { judgement }\end{array}$ & Risk of bias & $\begin{array}{l}\text { Applicability } \\
\text { concerns }\end{array}$ \\
\hline
\end{tabular}

DOMAIN 1: Patient Selection

Was a consecutive or random sample of patients enrolled?

Yes

Was a case-control design avoided? Yes

Did the study avoid inappropriate exclusions? Yes

Could the selection of patients have introduced bias?

Low risk

Are there concerns that the included patients and setting do not match the review question?

High

\section{DOMAIN 2: Index Test (Xpert MTB/RIF)}

Were the index test results interpreted without knowledge of the results of Yes the reference standard?

If a threshold was used, was it pre-specified? Yes

\section{Could the conduct or interpretation of the index test have introduced bias? \\ Low risk}

Are there concerns that the index test, its conduct, or interpretation differ from the review question?

\section{DOMAIN 2: Index Test (Xpert Ultra)}

\section{DOMAIN 3: Reference Standard}

Is the reference standards likely to correctly classify the target condition? Yes

Were the reference standard results interpreted without knowledge of the Yes results of the index tests? 
Che 2017 (Continued)

For rifampicin resistance testing, were the reference standard results inter- Unclear preted without knowledge of the results of the index test?

\section{Could the reference standard, its conduct, or its interpretation have in-} troduced bias?

Low risk

\section{Are there concerns that the target condition as defined by the reference} standard does not match the question?

Low concern

\section{DOMAIN 4: Flow and Timing}

Was there an appropriate interval between index test and reference stan- Yes dard?

Did all patients receive the same reference standard?

Yes

Were all patients included in the analysis?

Yes

Could the patient flow have introduced bias?

Low risk

Chen 2019

\section{Study characteristics}

\section{Patient Sampling}

Patient characteristics and setting
Cross-sectional, consecutive, prospective

Presenting signs and symptoms: patients who had symptoms suggestive of urinary tract TB or a urine abnormality

Age: mean 53 years (range, 19 to 85 )

Sex, female: $55 \%$

Children: no

HIV infection: $0 \%$

Clinical setting: multicentre, hospital-based

Past history of TB: $31 \%$

Participants on anti-TB treatment: no

Number of specimens evaluated: 302

Laboratory level: central

Country: China

World Bank Income Classification: middle income

High TB burden: yes

High TB/HIV burden: yes

High MDR-TB burden: yes 
Chen 2019 (Continued)

WHO SOP or manufacturer's protocol followed: yes

Target condition and reference standard(s)

Genitourinary TB

MGIT; CRS: culture or positive cystoscopy biopsy, or radiological signs

Speciation: yes

Decontamination: yes

Flow and timing

Comparative

Notes

Methodological quality

\begin{tabular}{lll}
\hline Item & $\begin{array}{l}\text { Authors' } \\
\text { judgement }\end{array}$ & $\begin{array}{c}\text { Risk of bias } \\
\text { Applicability } \\
\text { concerns }\end{array}$ \\
\hline
\end{tabular}

DOMAIN 1: Patient Selection

\begin{tabular}{lcc}
\hline Was a consecutive or random sample of patients enrolled? & Yes \\
\hline Was a case-control design avoided? & Yes \\
\hline Did the study avoid inappropriate exclusions? & Yes & Low risk \\
\hline Could the selection of patients have introduced bias? & \\
\hline $\begin{array}{l}\text { Are there concerns that the included patients and setting do not match } \\
\text { the review question? }\end{array}$
\end{tabular}

\section{DOMAIN 2: Index Test (Xpert MTB/RIF)}

Were the index test results interpreted without knowledge of the results of Yes the reference standard?

If a threshold was used, was it pre-specified? Yes

\section{Could the conduct or interpretation of the index test have introduced bias? \\ Low risk}

Are there concerns that the index test, its conduct, or interpretation differ from the review question?

\section{DOMAIN 2: Index Test (Xpert Ultra)}

\section{DOMAIN 3: Reference Standard}

Is the reference standards likely to correctly classify the target condition? Unclear

Were the reference standard results interpreted without knowledge of the Yes results of the index tests? 
Chen 2019 (Continued)

For rifampicin resistance testing, were the reference standard results interpreted without knowledge of the results of the index test?

\section{Could the reference standard, its conduct, or its interpretation have in- troduced bias? \\ Unclear risk}

\section{Are there concerns that the target condition as defined by the reference} standard does not match the question?

\section{DOMAIN 4: Flow and Timing}

Was there an appropriate interval between index test and reference stan- Yes dard?

Did all patients receive the same reference standard?

Yes

Were all patients included in the analysis?

Yes

Could the patient flow have introduced bias?

Low risk

Chin 2019

\section{Study characteristics}

\section{Patient Sampling}

Patient characteristics and setting
Cross-sectional, consecutive, prospective

Presenting signs and symptoms: patients with suspected TB meningitis admitted to the neurology ward

Age: adults, range 20 to 41 years

Sex, female: not reported

Children: not reported

HIV infection: $18 \%$

Clinical setting: tertiary care centre (inpatient)

Past history of TB: not reported

Participants on anti-TB treatment: 1 participant had received treatment

Number of specimens evaluated: 11

Laboratory level: central

Country: Uganda

World Bank Income Classification: low

High TB burden: no

High TB/HIV burden: yes

High MDR-TB burden: no 
Chin 2019 (Continued)

WHO SOP or manufacturer's protocol followed: no

$\begin{array}{ll}\text { Target condition and reference standard(s) } & \text { TB meningitis } \\ \text { MGIT } \\ \text { Speciation: not reported } \\ \text { Decontamination:no }\end{array}$

Flow and timing

Comparative

Notes "CSF $(2 \mathrm{ml})$ should be slowly pipetted directly into the Xpert Ultra cartridge. CSF should only be diluted with the manufacturer-supplied sample reagent if less than $2 \mathrm{ml}$ of CSF are available for Xpert Ultra testing." See the following article for full description of CSF processing details, Chin 2019a.

\section{Methodological quality}

\begin{tabular}{lll}
\hline Item & $\begin{array}{l}\text { Authors' } \\
\text { judgement }\end{array}$ & $\begin{array}{c}\text { Risk of bias } \\
\text { Applicability } \\
\text { concerns }\end{array}$ \\
\hline
\end{tabular}

\section{DOMAIN 1: Patient Selection}

Was a consecutive or random sample of patients enrolled? Yes

\begin{tabular}{lcc}
\hline Was a case-control design avoided? & Yes & Yes \\
\hline Did the study avoid inappropriate exclusions? & Low risk \\
\hline Could the selection of patients have introduced bias? & Low concern \\
\hline $\begin{array}{l}\text { Are there concerns that the included patients and setting do not match } \\
\text { the review question? }\end{array}$
\end{tabular}

DOMAIN 2: Index Test (Xpert MTB/RIF)

Were the index test results interpreted without knowledge of the results of Yes the reference standard?

If a threshold was used, was it pre-specified? Yes

Could the conduct or interpretation of the index test have introduced Low risk
bias?

\section{Are there concerns that the index test, its conduct, or interpretation dif-} fer from the review question?

\section{DOMAIN 2: Index Test (Xpert Ultra)}

Were the index test results interpreted without knowledge of the results of Yes the reference standard?

If a threshold was used, was it pre-specified? Yes 
Chin 2019 (Continued)

Could the conduct or interpretation of the index test have introduced

bias?

Are there concerns that the index test, its conduct, or interpretation dif-

fer from the review question?

\section{DOMAIN 3: Reference Standard}

Is the reference standards likely to correctly classify the target condition? Yes

Were the reference standard results interpreted without knowledge of the Yes results of the index tests?

For rifampicin resistance testing, were the reference standard results interpreted without knowledge of the results of the index test?

Could the reference standard, its conduct, or its interpretation have in-

Low risk troduced bias?

Are there concerns that the target condition as defined by the reference

Unclear standard does not match the question?

\section{DOMAIN 4: Flow and Timing}

Was there an appropriate interval between index test and reference stan- $\quad$ Yes dard?

Did all patients receive the same reference standard? Yes

Were all patients included in the analysis?

Yes

Could the patient flow have introduced bias?

Low risk

\section{Christopher 2013}

\section{Study characteristics}

Patient Sampling

Patient characteristics and setting
Cross-sectional, consecutive, prospective

Presenting signs and symptoms: clinical symptoms and radiographic evidence of a pleural effusion

Age: median 46 years (IQR 33 to 57 )

Sex, female: $20 \%$

Children: no

HIV infection: not reported

Clinical setting: tertiary care centre (Inpatient and outpatient)

Past history of TB: yes, $18 \%$

Patients on anti-TB treatment: not reported

Number of specimens evaluated against culture: 142 
Number of specimens evaluated against composite reference standard: 146

Laboratory level: central

Country: India

World Bank Income Classification: middle income

High TB burden: yes

High TB/HIV burden: yes

High MDR-TB burden: yes

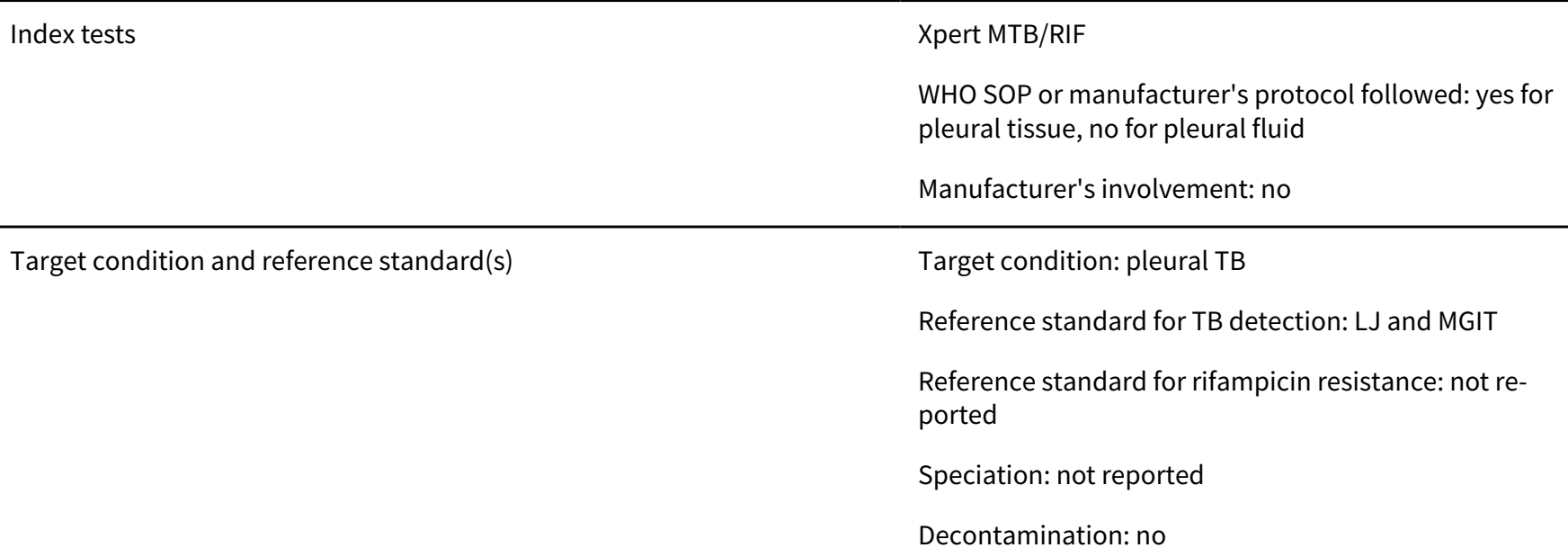

Flow and timing

Comparative

Notes

\section{Methodological quality}

\begin{tabular}{lll}
\hline Item & $\begin{array}{l}\text { Authors' judge- } \\
\text { ment }\end{array}$ & $\begin{array}{l}\text { Risk of bias } \\
\text { Applicability } \\
\text { concerns }\end{array}$ \\
\hline
\end{tabular}

DOMAIN 1: Patient Selection

\begin{tabular}{lcl}
\hline Was a consecutive or random sample of patients enrolled? & Yes & Yes \\
\hline Was a case-control design avoided? & Yes & Low risk \\
\hline Did the study avoid inappropriate exclusions? & \\
\hline Could the selection of patients have introduced bias? & Unclear \\
\hline $\begin{array}{l}\text { Are there concerns that the included patients and setting do not } \\
\text { match the review question? }\end{array}$
\end{tabular}

\section{DOMAIN 2: Index Test (Xpert MTB/RIF)}

Were the index test results interpreted without knowledge of the results of Yes the reference standard? 
Christopher 2013 (Continued)

\section{DOMAIN 2: Index Test (Xpert Ultra)}

\section{DOMAIN 3: Reference Standard}

Is the reference standards likely to correctly classify the target condition? Yes

Were the reference standard results interpreted without knowledge of the Yes results of the index tests?

For rifampicin resistance testing, were the reference standard results in- Unclear terpreted without knowledge of the results of the index test?

\section{Could the reference standard, its conduct, or its interpretation have Low risk introduced bias?}
Are there concerns that the target condition as defined by the refer- ence standard does not match the question?

\section{DOMAIN 4: Flow and Timing}

Was there an appropriate interval between index test and reference stan- Yes dard?

\begin{tabular}{lc}
\hline Did all patients receive the same reference standard? & Yes \\
\hline Were all patients included in the analysis? & Yes \\
\hline Could the patient flow have introduced bias? & Low risk \\
\hline
\end{tabular}

\section{Cresswell 2018}

\section{Study characteristics}

\section{Patient Sampling}

Patient characteristics and setting
Cohort, consecutive, prospective

Presenting signs and symptoms: patients with
headache and objective meningism
Age: median age 35 years (IQR 30 to 42 )
Sex, female: $39 \%$
Children: no
HIV infection: $4 \%$
Clinical setting: inpatient
Past history of TB: not reported

Xpert MTB/RIF Ultra and Xpert MTB/RIF assays for extrapulmonary tuberculosis and rifampicin resistance in adults (Review) 
Number of specimens evaluated: 118

Laboratory level: central

Country: Uganda

World Bank Income Classification: low income

High TB burden: no

High TB/HIV burden: yes

High MDR-TB burden: no

WHO SOP or manufacturer's protocol followed: not reported

Target condition and reference standard(s)

\section{TB meningitis}

\section{MGIT}

Speciation: not reported

Decontamination: no

\section{Flow and timing}

Comparative

Notes

Additonal information at clinicaltrials.gov/ct2/show/ NCT01075152

\section{Methodological quality}

\begin{tabular}{lll}
\hline Item & $\begin{array}{l}\text { Authors' } \\
\text { judgement }\end{array}$ & $\begin{array}{c}\text { Risk of bias } \\
\text { Applicability } \\
\text { concerns }\end{array}$ \\
\hline
\end{tabular}

\section{DOMAIN 1: Patient Selection}

\begin{tabular}{lcc}
\hline Was a consecutive or random sample of patients enrolled? & Yes \\
\hline Was a case-control design avoided? & Yes & Low risk \\
\hline Did the study avoid inappropriate exclusions? & \\
\hline Could the selection of patients have introduced bias?
\end{tabular}

Are there concerns that the included patients and setting do not match Low concern
the review question?

DOMAIN 2: Index Test (Xpert MTB/RIF)

Were the index test results interpreted without knowledge of the results of Yes the reference standard? 
Cresswell 2018 (Continued)

Could the conduct or interpretation of the index test have introduced

Low risk bias?

Are there concerns that the index test, its conduct, or interpretation dif-

Unclear fer from the review question?

\section{DOMAIN 2: Index Test (Xpert Ultra)}

\section{DOMAIN 3: Reference Standard}

Is the reference standards likely to correctly classify the target condition? Yes

Were the reference standard results interpreted without knowledge of the Yes results of the index tests?

For rifampicin resistance testing, were the reference standard results inter-

preted without knowledge of the results of the index test?

Could the reference standard, its conduct, or its interpretation have in- Low risk
troduced bias?

\section{Are there concerns that the target condition as defined by the reference} standard does not match the question?

\section{DOMAIN 4: Flow and Timing}

Was there an appropriate interval between index test and reference stan- Yes dard?

Did all patients receive the same reference standard? Yes

Were all patients included in the analysis? Yes

Could the patient flow have introduced bias? Low risk

Cresswell 2020

\section{Study characteristics}

Patient Sampling

Patient characteristics and setting
Cohort, consecutive, prospective

Presenting signs and symptoms: patients with suspected meningitis (headache for $>3$ days or altered mental status (Glasgow Coma Scale $<15$ ) with clinical signs of meningism at examination, i.e. neck stiffness or Kernig's sign

Age: median age 32 years (29 to 38 )

Sex, female: $42.6 \%$

Children: no

HIV infection: $96 \%$

Clinical setting: inpatient 
Past history of TB: not reported

Participants on anti-TB treatment: not reported

Number of specimens evaluated: 204

Laboratory level: central

Country: Uganda

World Bank Income Classification: low income

High TB burden: no

High TB/HIV burden: yes

High MDR-TB burden: no

Index tests

Xpert MTB/RIFand Xpert Ultra

WHO SOP or manufacturer's protocol followed: yes

Target condition and reference standard(s)

TB meningitis

MGIT

Speciation: yes

Decontamination: no

Flow and timing

\section{Comparative}

Notes

\section{Methodological quality}

\begin{tabular}{|c|c|c|c|}
\hline Item & $\begin{array}{l}\text { Authors' } \\
\text { judgement }\end{array}$ & Risk of bias & $\begin{array}{l}\text { Applicability } \\
\text { concerns }\end{array}$ \\
\hline
\end{tabular}

DOMAIN 1: Patient Selection

Was a consecutive or random sample of patients enrolled? Yes

Was a case-control design avoided? No

Did the study avoid inappropriate exclusions? Yes

Could the selection of patients have introduced bias?

Low risk

Are there concerns that the included patients and setting do not match the review question?

\section{DOMAIN 2: Index Test (Xpert MTB/RIF)}

Were the index test results interpreted without knowledge of the results of 
Cresswell 2020 (Continued)

Could the conduct or interpretation of the index test have introduced

Low risk

bias?

Are there concerns that the index test, its conduct, or interpretation dif-

Low concern fer from the review question?

\section{DOMAIN 2: Index Test (Xpert Ultra)}

Were the index test results interpreted without knowledge of the results of Yes the reference standard?

If a threshold was used, was it pre-specified?

Yes

\section{Could the conduct or interpretation of the index test have introduced bias?

Are there concerns that the index test, its conduct, or interpretation differ from the review question?

\section{DOMAIN 3: Reference Standard}

Is the reference standards likely to correctly classify the target condition? Yes

Were the reference standard results interpreted without knowledge of the Yes results of the index tests?

For rifampicin resistance testing, were the reference standard results inter- Yes preted without knowledge of the results of the index test?

Could the reference standard, its conduct, or its interpretation have introduced bias?

\section{Are there concerns that the target condition as defined by the reference standard does not match the question?}

\section{DOMAIN 4: Flow and Timing}

Was there an appropriate interval between index test and reference stan- Yes dard?

Did all patients receive the same reference standard? Yes

Were all patients included in the analysis? Yes

Could the patient flow have introduced bias?

Low risk

Dhasmana 2014

\section{Study characteristics}

Patient Sampling

Cross-sectional, consecutive, prospective

Patient characteristics and setting

Presenting signs and symptoms: all participants undergoing endobronchial ultrasound (EBUS) for mediastinal lymphadenopathy 
Sex, female: $37 \%$

Children: no

HIV infection: $7 \%$

Clinical setting: tertiary care centre (inpatient and outpatient)

Past history of TB: not reported

Patients on anti-TB treatment: no

Number of specimens evaluated: 116

Laboratory level: central

Country: United Kingdom

World Bank Income Classification: high income

High TB burden: no

High TB/HIV burden: no

High MDR-TB burden: no

WHO SOP or manufacturer's protocol followed: no

Manufacturer's involvement: no

Decontamination: yes, NALC-NaOH

Flow and timing

Comparative

Notes

\section{Methodological quality}

\begin{tabular}{|c|c|c|c|}
\hline Item & $\begin{array}{l}\text { Authors' } \\
\text { judgement }\end{array}$ & Risk of bias & $\begin{array}{l}\text { Applicability } \\
\text { concerns }\end{array}$ \\
\hline
\end{tabular}

DOMAIN 1: Patient Selection

Was a consecutive or random sample of patients enrolled?

Was a case-control design avoided?
Yes

Yes 
Dhasmana 2014 (Continued)

Did the study avoid inappropriate exclusions?

Yes

\section{Could the selection of patients have introduced bias?}

Low risk

Are there concerns that the included patients and setting do not match

Unclear the review question?

\section{DOMAIN 2: Index Test (Xpert MTB/RIF)}

Were the index test results interpreted without knowledge of the results of Yes the reference standard?

If a threshold was used, was it pre-specified? Yes

\section{Could the conduct or interpretation of the index test have introduced bias?}

Are there concerns that the index test, its conduct, or interpretation dif-

fer from the review question?

\section{DOMAIN 2: Index Test (Xpert Ultra)}

DOMAIN 3: Reference Standard

Is the reference standards likely to correctly classify the target condition? Unclear

Were the reference standard results interpreted without knowledge of the Yes results of the index tests?

For rifampicin resistance testing, were the reference standard results inter- Yes preted without knowledge of the results of the index test?

\section{Could the reference standard, its conduct, or its interpretation have in- Unclear risk} troduced bias?

Are there concerns that the target condition as defined by the reference standard does not match the question?

\section{DOMAIN 4: Flow and Timing}

Was there an appropriate interval between index test and reference stan- Yes dard?

Did all patients receive the same reference standard?

Yes

Were all patients included in the analysis?

Yes

\section{Could the patient flow have introduced bias?}

Low risk

Dhooria 2016

\section{Study characteristics}


Dhooria 2016 (Continued)

Patient characteristics and setting
Presenting signs and symptoms: patients with enlarged mediastinal or hilar lymph nodes $(\geq 1 \mathrm{~cm}$ in short axis) on computed tomography of the chest who underwent EBUS-guided transbronchial needle aspiration

Age: median 40 years, range 30 to 53 years

Sex, female: $43 \%$

Children: no

HIV infection: $0 \%$

Clinical setting: tertiary care centre (outpatient)

Past history of TB: not reported

Patients on anti-TB treatment: no

Number of specimens evaluated: 147

Laboratory level: central

Country: India

World Bank Income Classification: middle income

High TB burden: yes

High TB/HIV burden: yes

High MDR-TB burden: yes

Index tests

Xpert MTB/RIF

WHO SOP or manufacturer's protocol followed: yes

Manufacturer's involvement: no

Target condition and reference standard(s)

Target condition: lymph node TB

Reference standard for TB detection: MGIT

Reference standard for rifampicin resistance: not reported

Speciation: not reported

Decontamination: no

\section{Flow and timing}

Comparative

Notes

\section{Methodological quality}

\begin{tabular}{|c|c|c|c|}
\hline Item & $\begin{array}{l}\text { Authors' } \\
\text { judgement }\end{array}$ & Risk of bias & $\begin{array}{l}\text { Applicability } \\
\text { concerns }\end{array}$ \\
\hline
\end{tabular}

\section{DOMAIN 1: Patient Selection}


Dhooria 2016 (Continued)

Was a consecutive or random sample of patients enrolled?

Was a case-control design avoided? Yes

Did the study avoid inappropriate exclusions? Yes

\section{Could the selection of patients have introduced bias?}

Low risk

Are there concerns that the included patients and setting do not match

Unclear the review question?

DOMAIN 2: Index Test (Xpert MTB/RIF)

Were the index test results interpreted without knowledge of the results of Yes the reference standard?

If a threshold was used, was it pre-specified? Yes

\section{Could the conduct or interpretation of the index test have introduced} bias?

Are there concerns that the index test, its conduct, or interpretation dif-

fer from the review question?

\section{DOMAIN 2: Index Test (Xpert Ultra)}

\section{DOMAIN 3: Reference Standard}

Is the reference standards likely to correctly classify the target condition? Yes

Were the reference standard results interpreted without knowledge of the Yes results of the index tests?

For rifampicin resistance testing, were the reference standard results inter- Unclear preted without knowledge of the results of the index test?

\section{Could the reference standard, its conduct, or its interpretation have introduced bias?

\section{Are there concerns that the target condition as defined by the reference} standard does not match the question?

\section{DOMAIN 4: Flow and Timing}

Was there an appropriate interval between index test and reference stan- Yes dard?

Did all patients receive the same reference standard?

Yes

Were all patients included in the analysis?

Yes

\section{Could the patient flow have introduced bias?}

Low risk 
Donovan 2020

\section{Study characteristics}

\section{Patient Sampling}

Patient characteristics and setting
Cross-sectional, random, prospective

Presenting signs and symptoms: patients aged 16 years or older with suspected tuberculous meningitis based on clinical and CSF findings (clear or mildly cloudy CSF, plus $>5$ days of symptoms consistent with tuberculous meningitis 8 or low CSF glucose or raised CSF lactate concentrations)

Age: median age 42 (31 to 57 )

Sex, female: $40 \%$

Children: no

HIV infection: $17 \%$

Clinical setting: inpatient

Past history of TB: not reported

Participants on anti-TB treatment: not reported

Number of specimens evaluated: 205

Laboratory level: central

Country: Vietnam

World Bank Income Classification: middle income

High TB burden: yes

High TB/HIV burden: no

High MDR-TB burden: yes

Index tests

Xpert MTB/RIF and Xpert Ultra

WHO SOP or manufacturer's protocol followed: yes

MGIT

Speciation: yes

Decontamination: no

Flow and timing

Comparative

Notes

Methodological quality

\begin{tabular}{lll} 
Item & $\begin{array}{l}\text { Authors' } \\
\text { judgement }\end{array}$ & Risk of bias $\begin{array}{l}\text { Applicability } \\
\text { concerns }\end{array}$ \\
\hline
\end{tabular}


Donovan 2020 (Continued)

DOMAIN 1: Patient Selection

\begin{tabular}{lc}
\hline Was a consecutive or random sample of patients enrolled? & Yes \\
\hline Was a case-control design avoided? & Yes \\
\hline Did the study avoid inappropriate exclusions? & Yes \\
\hline Could the selection of patients have introduced bias? & Low risk \\
\hline $\begin{array}{l}\text { Are there concerns that the included patients and setting do not match } \\
\text { the review question? }\end{array}$
\end{tabular}

\title{
DOMAIN 2: Index Test (Xpert MTB/RIF)
}

Were the index test results interpreted without knowledge of the results of Yes the reference standard?

If a threshold was used, was it pre-specified?

Yes

\author{
Could the conduct or interpretation of the index test have introduced \\ bias?

Are there concerns that the index test, its conduct, or interpretation dif-

fer from the review question?

DOMAIN 2: Index Test (Xpert Ultra)

Were the index test results interpreted without knowledge of the results of Yes the reference standard?

If a threshold was used, was it pre-specified? Yes

\begin{tabular}{|c|c|}
\hline $\begin{array}{l}\text { Could the conduct or interpretation of the index test have introduced } \\
\text { bias? }\end{array}$ & Low risk \\
\hline
\end{tabular}

bias?

Are there concerns that the index test, its conduct, or interpretation dif-

fer from the review question?

\section{DOMAIN 3: Reference Standard}

Is the reference standards likely to correctly classify the target condition? Yes

Were the reference standard results interpreted without knowledge of the Yes results of the index tests?

For rifampicin resistance testing, were the reference standard results inter- Yes preted without knowledge of the results of the index test?

Could the reference standard, its conduct, or its interpretation have in- Low risk troduced bias?

Are there concerns that the target condition as defined by the reference standard does not match the question?

DOMAIN 4: Flow and Timing 
Donovan 2020 (Continued)

Was there an appropriate interval between index test and reference stan- Yes dard?

Did all patients receive the same reference standard?

Yes

Were all patients included in the analysis?

Yes

Could the patient flow have introduced bias?

Low risk

Du 2015

\section{Study characteristics}

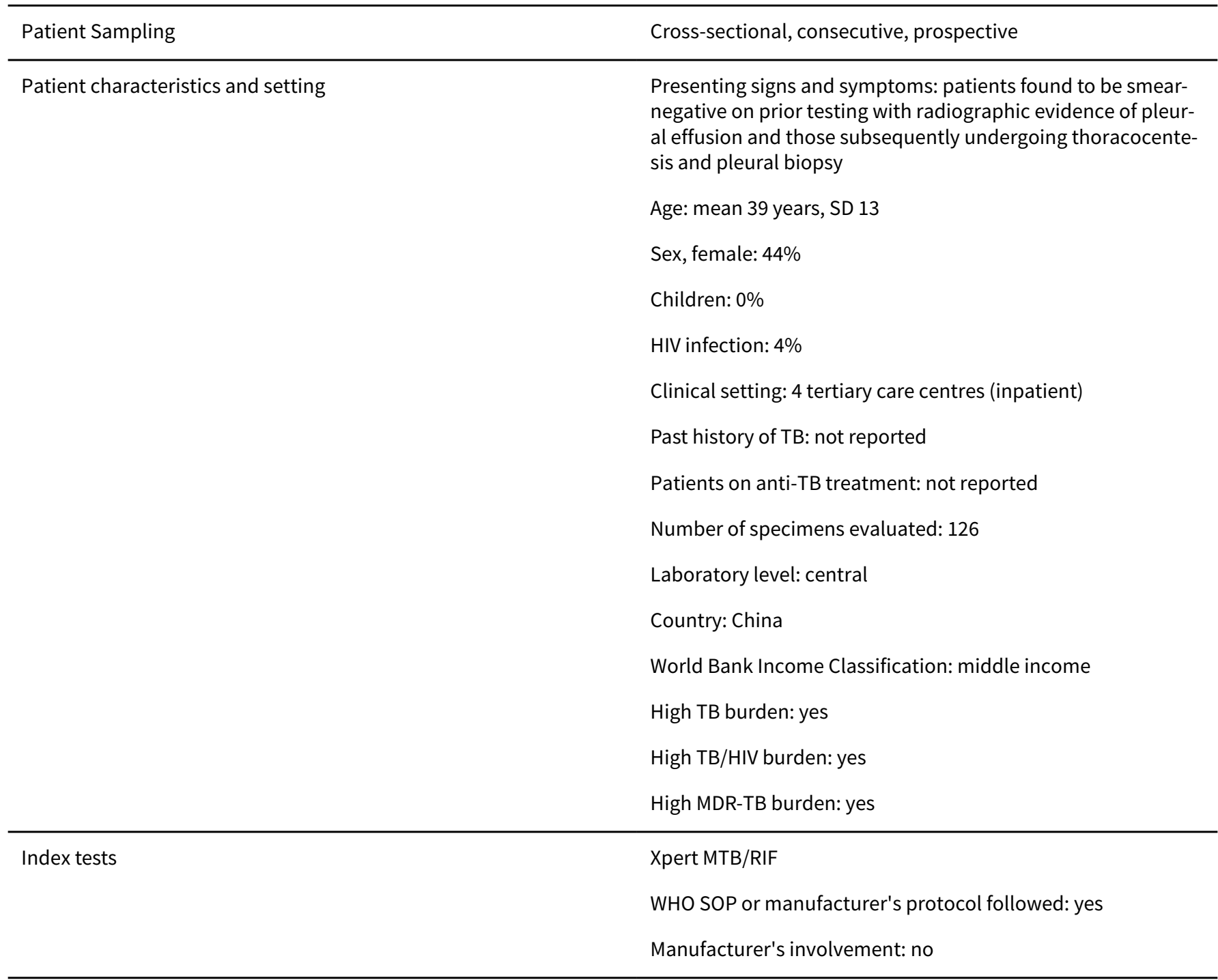

Target condition and reference standard(s)

Target condition: pleural TB

Reference standard for TB detection: LJ and MGIT

Reference standard for rifampicin resistance: MGIT-DST

Speciation: yes 
Du 2015 (Continued)

Decontamination: yes, NALC-NaOH

Flow and timing

Comparative

Notes

Study included specimens found to be smear-negative on prior testing. In the present study, 4 specimens were smear-positive specimens for pleural fluid and 15 were smear-positive for pleural tissue

The reference standard for both pleural fluid and pleural tissue was pleural biopsy culture

\section{Methodological quality}

\begin{tabular}{|c|c|c|c|}
\hline Item & $\begin{array}{l}\text { Authors' judge- } \\
\text { ment }\end{array}$ & Risk of bias & $\begin{array}{l}\text { Applicability con- } \\
\text { cerns }\end{array}$ \\
\hline
\end{tabular}

\section{DOMAIN 1: Patient Selection}

\begin{tabular}{lc}
\hline Was a consecutive or random sample of patients enrolled? & Yes \\
\hline Was a case-control design avoided? & Yes \\
\hline Did the study avoid inappropriate exclusions? & No
\end{tabular}

\begin{tabular}{ll}
\hline Could the selection of patients have introduced bias? High risk \\
\hline
\end{tabular}

Are there concerns that the included patients and setting do not match the review question?

\section{DOMAIN 2: Index Test (Xpert MTB/RIF)}

Were the index test results interpreted without knowledge of the re- Yes sults of the reference standard?

\begin{tabular}{l}
\hline If a threshold was used, was it pre-specified? \\
$\begin{array}{l}\text { Could the conduct or interpretation of the index test have intro- } \\
\text { duced bias? }\end{array}$ \\
\hline $\begin{array}{l}\text { Are there concerns that the index test, its conduct, or interpre- } \\
\text { tation differ from the review question? }\end{array}$ \\
\hline DOMAIN 2: Index Test (Xpert Ultra)
\end{tabular}

\section{DOMAIN 3: Reference Standard} Is the reference standards likely to correctly classify the target con- Unclear
dition?

Were the reference standard results interpreted without knowledge Yes of the results of the index tests?

For rifampicin resistance testing, were the reference standard re- Yes sults interpreted without knowledge of the results of the index test? 
Du 2015 (Continued)

Could the reference standard, its conduct, or its interpretation

Unclear risk have introduced bias?

Are there concerns that the target condition as defined by the

Low concern reference standard does not match the question?

\section{DOMAIN 4: Flow and Timing}

Was there an appropriate interval between index test and reference Yes standard?

\begin{tabular}{lc}
\hline Did all patients receive the same reference standard? & Yes \\
\hline Were all patients included in the analysis? & Yes \\
\hline Could the patient flow have introduced bias?
\end{tabular}

El-Din 2019

\section{Study characteristics}

Patient Sampling Cross-sectional. consecutive, prospective

Patient characteristics and setting

Presenting signs and symptoms: patients with suspected pleural TB based on clinical history and radiologic evidence of pleural effusion

Age: 32.7 years \pm 13.6

Sex, female: $31 \%$

Children: no

HIV infection: not reported

Clinical setting: not reported

Past history of TB: not reported

Participants on anti-TB treatment: not reported

Number of specimens evaluated: not reported

Laboratory level: not reported

Country: Egypt

World Bank Income Classification: middle income

High TB burden: no

High TB/HIV burden: no

High MDR-TB burden: no

Specimens processed according to manufacturer's instructions 
El-Din 2019 (Continued)

Target condition and reference standard(s)
Pleural TB

Confirmed TB was defined if acid-fast bacilli were detected by any mean (microscopic evaluation/mycobacterial culture (type not reported)) of either pleural tissue or pleural fluid

Speciation: not reported

Decontamination: no

Flow and timing

Comparative

Notes

Methodological quality

\begin{tabular}{lll}
\hline Item & $\begin{array}{l}\text { Authors' } \\
\text { judgement }\end{array}$ & $\begin{array}{l}\text { Risk of bias } \\
\text { Applicability } \\
\text { concerns }\end{array}$ \\
\hline
\end{tabular}

\section{DOMAIN 1: Patient Selection}

\begin{tabular}{lcl}
\hline Was a consecutive or random sample of patients enrolled? & Yes \\
\hline Was a case-control design avoided? & Yes \\
\hline Did the study avoid inappropriate exclusions? & Low risk \\
\hline Could the selection of patients have introduced bias?
\end{tabular}

Are there concerns that the included patients and setting do not match Unclear
the review question?

DOMAIN 2: Index Test (Xpert MTB/RIF)

Were the index test results interpreted without knowledge of the results of Yes the reference standard?

If a threshold was used, was it pre-specified? Yes

Could the conduct or interpretation of the index test have introduced Low risk
bias?

bias?

Are there concerns that the index test, its conduct, or interpretation differ from the review question?

\section{DOMAIN 2: Index Test (Xpert Ultra)}

\section{DOMAIN 3: Reference Standard}

\begin{tabular}{l}
\hline Is the reference standards likely to correctly classify the target condition? Yes \\
\hline $\begin{array}{l}\text { Were the reference standard results interpreted without knowledge of the Unclear } \\
\text { results of the index tests? }\end{array}$
\end{tabular} 
El-Din 2019 (Continued)

For rifampicin resistance testing, were the reference standard results interpreted without knowledge of the results of the index test?

\section{Could the reference standard, its conduct, or its interpretation have in-} troduced bias?

\section{Are there concerns that the target condition as defined by the reference} standard does not match the question?

Unclear risk

\section{DOMAIN 4: Flow and Timing}

Was there an appropriate interval between index test and reference stan- Yes dard?

Did all patients receive the same reference standard?

Yes

Were all patients included in the analysis?

Yes

Could the patient flow have introduced bias?

Low risk

Unclear

Low risk

Feasey 2013

\section{Study characteristics}

Patient Sampling

Patient characteristics and setting
Cross-sectional, consecutive, prospective

Presenting signs and symptoms: HIV-infected patients with clinical suspicion of tuberculosis

Age: mean 37 years, SD 11 years

Sex, female: $33 \%$

Children: no

HIV infection: $100 \%$

Clinical setting: tertiary care centre

Past history of TB: no

Patients on anti-TB treatment: no

Number of specimens evaluated: 74

Laboratory level: central

Country: Malawi

World Bank Income Classification: low income

High TB burden: no

High TB/HIV burden: no

High MDR-TB burden: no
Xpert MTB/RIF

WHO SOP or manufacturer's protocol followed: no 
Feasey 2013 (Continued)

Manufacturer's involvement: no

Target condition and reference standard(s)

Target condition: disseminated TB (blood)

Reference standard for TB detection: Bactec Myco/F Lytic culture

Reference standard for rifampicin resistance: not reported

Speciation: yes

Decontamination: yes, NALC-NaOH for sputum specimens

Flow and timing

Comparative

Notes

Methodological quality

\begin{tabular}{lll}
\hline Item & $\begin{array}{l}\text { Authors' } \\
\text { judgement }\end{array}$ & $\begin{array}{c}\text { Risk of bias } \\
\text { Applicability } \\
\text { concerns }\end{array}$ \\
\hline
\end{tabular}

DOMAIN 1: Patient Selection

\begin{tabular}{lcc}
\hline Was a consecutive or random sample of patients enrolled? & Yes \\
\hline Was a case-control design avoided? & Yes \\
\hline Did the study avoid inappropriate exclusions? & Yes & Low risk \\
\hline Could the selection of patients have introduced bias? & Unclear \\
\hline $\begin{array}{l}\text { Are there concerns that the included patients and setting do not match } \\
\text { the review question? }\end{array}$ & \\
\hline DOMAIN 2: Index Test (Xpert MTB/RIF)
\end{tabular}

Were the index test results interpreted without knowledge of the results of Yes the reference standard?

If a threshold was used, was it pre-specified? Yes

Could the conduct or interpretation of the index test have introduced Low risk
bias?

Are there concerns that the index test, its conduct, or interpretation differ from the review question?

DOMAIN 2: Index Test (Xpert Ultra)

\section{DOMAIN 3: Reference Standard}

Is the reference standards likely to correctly classify the target condition?

Yes 
Feasey 2013 (Continued)

Were the reference standard results interpreted without knowledge of the Yes results of the index tests?

For rifampicin resistance testing, were the reference standard results inter- Unclear preted without knowledge of the results of the index test?

\section{Could the reference standard, its conduct, or its interpretation have in- Low risk troduced bias?}

\section{Are there concerns that the target condition as defined by the reference} standard does not match the question?

\section{DOMAIN 4: Flow and Timing}

Was there an appropriate interval between index test and reference standard?

\begin{tabular}{ll}
\hline Did all patients receive the same reference standard? Yes \\
\hline
\end{tabular}

Were all patients included in the analysis? Yes

\section{Could the patient flow have introduced bias?}

Low risk

Friedrich 2011

\section{Study characteristics}

\section{Patient Sampling}

Patient characteristics and setting
Cross-sectional, consecutive, prospective

Presenting signs and symptoms: patients with undiagnosed pleural effusion and high clinical suspicion of pleural TB

Age: not reported

Sex, female: $36 \%$

Children: $0 \%$

HIV infection: $28 \%$

Clinical setting: tertiary care centre (inpatient and outpatient)

Past history of TB: not reported

Patients on anti-TB treatment: not reported

Number of specimens evaluated against culture: 24

Number of specimens evaluated against composite reference standard: 25

Laboratory level: central

Country: South Africa

World Bank Income Classification: middle income 
Friedrich 2011 (Continued)

$$
\begin{aligned}
& \text { High TB burden: yes } \\
& \text { High TB/HIV burden: yes } \\
& \text { High MDR-TB burden: yes }
\end{aligned}
$$

Index tests

\section{Xpert MTB/RIF}

WHO SOP or manufacturer's protocol followed: no

Manufacturer's involvement: no

Target condition: pleural TB

Reference standard for TB detection: MGIT

Reference standard for rifampicin resistance: MGIT

Speciation: yes

Decontamination: yes, NALC-NaOH

Flow and timing

\section{Comparative}

Notes

\section{Methodological quality}

\begin{tabular}{lll}
\hline Item & $\begin{array}{l}\text { Authors' } \\
\text { judgement }\end{array}$ & Risk of bias Applicability \\
concerns
\end{tabular}

\section{DOMAIN 1: Patient Selection}

Was a consecutive or random sample of patients enrolled? Yes

\begin{tabular}{ll}
\hline Was a case-control design avoided? & Yes \\
\hline
\end{tabular}

Did the study avoid inappropriate exclusions?

Yes

\section{Could the selection of patients have introduced bias?}

Low risk

Are there concerns that the included patients and setting do not match
the review question?

\section{DOMAIN 2: Index Test (Xpert MTB/RIF)}

Were the index test results interpreted without knowledge of the results of Yes the reference standard?

If a threshold was used, was it pre-specified? Yes

Could the conduct or interpretation of the index test have introduced bias?

Are there concerns that the index test, its conduct, or interpretation differ from the review question? 
Friedrich 2011 (Continued)

DOMAIN 2: Index Test (Xpert Ultra)

\section{DOMAIN 3: Reference Standard}

Is the reference standards likely to correctly classify the target condition? Unclear

Were the reference standard results interpreted without knowledge of the Yes results of the index tests?

For rifampicin resistance testing, were the reference standard results inter- Yes

preted without knowledge of the results of the index test?
Could the reference standard, its conduct, or its interpretation have in-
Unclear risk troduced bias?
Are there concerns that the target condition as defined by the reference standard does not match the question?

\section{DOMAIN 4: Flow and Timing}

Was there an appropriate interval between index test and reference stan- Yes
dard?

Did all patients receive the same reference standard? Yes

Were all patients included in the analysis?

Yes

\section{Study characteristics}

\begin{tabular}{|c|c|}
\hline Patient Sampling & Cross-sectional, consecutive, prospective \\
\hline \multirow[t]{11}{*}{ Patient characteristics and setting } & $\begin{array}{l}\text { Presenting signs and symptoms: patients with clinical } \\
\text { suspicion of TB }\end{array}$ \\
\hline & Age: mean 32 years, range 3 to 79 years \\
\hline & Sex, female: $68 \%$ \\
\hline & Children: $13 \%$ \\
\hline & HIV infection: no \\
\hline & $\begin{array}{l}\text { Clinical setting: tertiary care centre (inpatient and } \\
\text { outpatient) }\end{array}$ \\
\hline & Past history of TB: $18 \%$ \\
\hline & Patients on anti-TB treatment: yes, $3 \%$ \\
\hline & Number of specimens evaluated: 174 \\
\hline & Laboratory level: central \\
\hline & Country: Tunisia \\
\hline
\end{tabular}


High TB burden: no

High TB/HIV burden: no

High MDR-TB burden: no

WHO SOP or manufacturer's protocol followed: yes

Manufacturer's involvement: no

Target condition and reference standard(s)

\author{
Target condition: lymph node TB \\ Reference standard for TB detection: LJ and MGIT \\ Reference standard for rifampicin resistance: MGIT- \\ DST \\ Speciation: yes \\ Decontamination: yes, NALC-NaOH
}

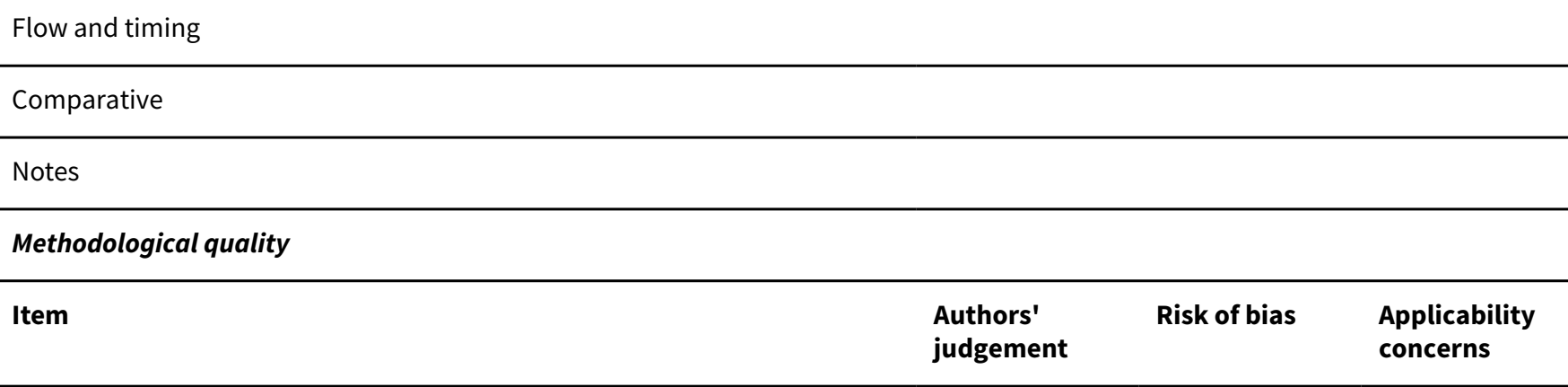

\title{
DOMAIN 1: Patient Selection
}

\begin{tabular}{lcl}
\hline Was a consecutive or random sample of patients enrolled? & Yes \\
\hline Was a case-control design avoided? & Yes & Yes \\
\hline Did the study avoid inappropriate exclusions? & Low risk \\
\hline Could the selection of patients have introduced bias? & \\
\hline $\begin{array}{l}\text { Are there concerns that the included patients and setting do not match } \\
\text { the review question? }\end{array}$
\end{tabular}

\section{DOMAIN 2: Index Test (Xpert MTB/RIF)}

Were the index test results interpreted without knowledge of the results of Yes the reference standard?

\section{Could the conduct or interpretation of the index test have introduced} bias? 
Ghariani 2015 (Continued)

Are there concerns that the index test, its conduct, or interpretation differ from the review question?

\section{DOMAIN 2: Index Test (Xpert Ultra)}

\section{DOMAIN 3: Reference Standard}

Is the reference standards likely to correctly classify the target condition? Unclear

Were the reference standard results interpreted without knowledge of the Yes results of the index tests?

For rifampicin resistance testing, were the reference standard results inter- Yes preted without knowledge of the results of the index test?

\begin{tabular}{|c|c|}
\hline $\begin{array}{l}\text { Could the reference standard, its conduct, or its interpretation have in- } \\
\text { troduced bias? }\end{array}$ & Unclear risk \\
\hline
\end{tabular}

Are there concerns that the target condition as defined by the reference

Low concern standard does not match the question?

\section{DOMAIN 4: Flow and Timing}

Was there an appropriate interval between index test and reference stan- Yes dard?

Did all patients receive the same reference standard? Yes

Were all patients included in the analysis? Yes

\section{Study characteristics}

Patient Sampling

Cross-sectional, manner of participant selection not reported, prospective

Patient characteristics and setting

\author{
Presenting signs and symptoms: patients with suspi- \\ cion of bone and joint TB \\ Age: median 42 years for TB patients, range 18 to 82 \\ years \\ Sex, female: $54 \%$ \\ Children: no \\ HIV infection: not reported \\ Clinical setting: tertiary care centre (inpatient) \\ Past history of TB: not reported \\ Patients on anti-TB treatment: yes, 100\% \\ Number of specimens evaluated: 60
}

Xpert MTB/RIF Ultra and Xpert MTB/RIF assays for extrapulmonary tuberculosis and rifampicin resistance in adults (Review) 
Gu 2015 (Continued)

\author{
Laboratory level: central \\ Country: China \\ World Bank Income Classification: middle income \\ High TB burden: yes \\ High TB/HIV burden: yes \\ High MDR-TB burden: yes
}

\begin{tabular}{ll}
\hline Index tests & Xpert MTB/RIF \\
& WHO SOP or manufacturer's protocol: yes \\
& Manufacturer's involvement: no \\
\hline Target condition and reference standard(s) & Target condition: bone and joint TB \\
& $\begin{array}{l}\text { Reference standard for TB detection: MGIT } \\
\text { Reference standard for rifampicin resistance: MGIT- } \\
\text { DST } \\
\text { Speciation: yes } \\
\text { Decontamination: yes, } \mathrm{NALC}-\mathrm{NaOH}\end{array}$ \\
\hline
\end{tabular}

Flow and timing

Comparative

Notes

Methodological quality

\begin{tabular}{lll}
\hline Item & $\begin{array}{l}\text { Authors' } \\
\text { judgement }\end{array}$ & Risk of bias $\begin{array}{c}\text { Applicability } \\
\text { concerns }\end{array}$ \\
\hline
\end{tabular}

DOMAIN 1: Patient Selection

\begin{tabular}{lcl}
\hline Was a consecutive or random sample of patients enrolled? & Unclear \\
\hline Was a case-control design avoided? & Yes & Yes \\
\hline Did the study avoid inappropriate exclusions? & Unclear risk \\
\hline Could the selection of patients have introduced bias? & High \\
\hline $\begin{array}{l}\text { Are there concerns that the included patients and setting do not match } \\
\text { the review question? }\end{array}$ & \\
\hline
\end{tabular}

\title{
DOMAIN 2: Index Test (Xpert MTB/RIF)
}

Were the index test results interpreted without knowledge of the results of Yes the reference standard?

If a threshold was used, was it pre-specified?

Yes 
Gu 2015 (Continued)

Could the conduct or interpretation of the index test have introduced

bias?

Are there concerns that the index test, its conduct, or interpretation dif-

Low concern

fer from the review question?

\section{DOMAIN 2: Index Test (Xpert Ultra)}

\section{DOMAIN 3: Reference Standard}

Is the reference standards likely to correctly classify the target condition? Unclear

Were the reference standard results interpreted without knowledge of the Yes results of the index tests?

For rifampicin resistance testing, were the reference standard results inter- Yes

preted without knowledge of the results of the index test?

\section{Could the reference standard, its conduct, or its interpretation have in- Unclear risk troduced bias?}

Are there concerns that the target condition as defined by the reference

Low concern standard does not match the question?

\section{DOMAIN 4: Flow and Timing}

Was there an appropriate interval between index test and reference stan- $\quad$ Yes dard?

Did all patients receive the same reference standard? Yes

Were all patients included in the analysis? Yes

Could the patient flow have introduced bias?

Low risk

Hanif 2011

\section{Study characteristics}

Patient Sampling Cross-sectional, consecutive, prospective

Patient characteristics and setting

Presenting signs and symptoms: patients with suspicion of TB due to symptoms such as fever, cough or weight loss or both, or because they were not responding to initial therapy for other diseases

Age: range 20 to 57 years

Sex, female: $39 \%$

Children: no

HIV infection: no

Clinical setting: national reference laboratory

Past history of TB: not reported 
Hanif 2011 (Continued)

Patients on anti-TB treatment: not reported

Number of specimens evaluated: 29

Laboratory level: central

Country: Kuwait

World Bank Income Classification: middle income

High TB burden: no

High TB/HIV burden: no

High MDR-TB burden: no

\begin{tabular}{ll}
\hline Index tests & Xpert MTB/RIF \\
& $\begin{array}{l}\text { WHO SOP or manufacturer's protocol: yes for lymph node } \\
\text { aspirate, pleural fluid, and urine; no for CSF } \\
\text { Manufacturer's involvement: no }\end{array}$ \\
\hline Target condition and reference standard(s) & $\begin{array}{l}\text { Target condition: TB meningitis, lymph node TB, pleural } \\
\text { TB, genitourinary TB }\end{array}$ \\
Reference standard for TB detection: LJ and MGIT \\
Reference standard for rifampicin resistance: LJ-DST and \\
MGIT-DST \\
Speciation: yes \\
Decontamination: no
\end{tabular}

Flow and timing

Comparative

Notes

\section{Methodological quality}

\begin{tabular}{lll}
\hline Item & $\begin{array}{l}\text { Authors' judge- } \\
\text { ment }\end{array}$ & $\begin{array}{l}\text { Risk of bias } \\
\text { Applicability } \\
\text { concerns }\end{array}$ \\
\hline
\end{tabular}

DOMAIN 1: Patient Selection

\begin{tabular}{lcc}
\hline Was a consecutive or random sample of patients enrolled? & Yes & Yes \\
\hline Was a case-control design avoided? & Yes & Low risk \\
\hline Did the study avoid inappropriate exclusions? & \\
\hline Could the selection of patients have introduced bias? & Unclear \\
\hline $\begin{array}{l}\text { Are there concerns that the included patients and setting do not } \\
\text { match the review question? }\end{array}$
\end{tabular}

\section{DOMAIN 2: Index Test (Xpert MTB/RIF)}


Hanif 2011 (Continued)

Were the index test results interpreted without knowledge of the results Yes of the reference standard?

If a threshold was used, was it pre-specified?

Yes

\section{Could the conduct or interpretation of the index test have intro- duced bias? \\ Low risk}

Are there concerns that the index test, its conduct, or interpretation
differ from the review question?

DOMAIN 2: Index Test (Xpert Ultra)

\section{DOMAIN 3: Reference Standard}

Is the reference standards likely to correctly classify the target condi- $\quad$ Yes tion?

Were the reference standard results interpreted without knowledge of No the results of the index tests?

For rifampicin resistance testing, were the reference standard results Yes interpreted without knowledge of the results of the index test?

Could the reference standard, its conduct, or its interpretation have High risk
introduced bias?
introduced bias?

\section{Are there concerns that the target condition as defined by the refer-} ence standard does not match the question?

\section{DOMAIN 4: Flow and Timing}

Was there an appropriate interval between index test and reference Yes standard?

\begin{tabular}{lc}
\hline Did all patients receive the same reference standard? & Yes \\
\hline Were all patients included in the analysis? & Yes \\
\hline
\end{tabular}

\section{Could the patient flow have introduced bias?}

Low risk

Heemskerk 2018

\section{Study characteristics}

\begin{tabular}{ll}
\hline Patient Sampling & Cross-sectional, consecutive, prospective \\
\hline Patient characteristics and setting & $\begin{array}{l}\text { Presenting signs and symptoms: patients who were of- } \\
\text { fered lumbar puncture as a part of routine care for sus- } \\
\text { pected brain infection }\end{array}$ \\
Age: $\geq 18$ years; median 37 years (IQR 28 to 50) \\
Sex, female: $43 \%$ \\
Children: no
\end{tabular}


Clinical setting: multicentre, hospital-based (both referral and local)

Past history of TB:

Participants on anti-TB treatment:

Number of specimens evaluated: 610

Laboratory level: central in South Africa

Country: South Africa, Vietnam, Indonesia

World Bank Income Classification: middle

High TB burden: South Africa yes; Vietnam yes; Indonesia yes

High TB/HIV burden: South Africa yes; Vietnam no; Indonesia yes

High MDR-TB burden: South Africa yes; Vietnam yes; Indonesia yes

WHO SOP or manufacturer's protocol followed: no (as performed in Nhu 2014)

Decontamination: no

Flow and timing

Comparative

Notes

Methodological quality

\begin{tabular}{|c|c|c|c|}
\hline Item & $\begin{array}{l}\text { Authors' judge- } \\
\text { ment }\end{array}$ & Risk of bias & $\begin{array}{l}\text { Applicability } \\
\text { concerns }\end{array}$ \\
\hline
\end{tabular}

\section{DOMAIN 1: Patient Selection}

\begin{tabular}{lcl}
\hline Was a consecutive or random sample of patients enrolled? & Yes & Yes \\
\hline Was a case-control design avoided? & Yes & Low risk \\
\hline Did the study avoid inappropriate exclusions? & \\
\hline Could the selection of patients have introduced bias?
\end{tabular}


Heemskerk 2018 (Continued)

Are there concerns that the included patients and setting do not match the review question?

\section{DOMAIN 2: Index Test (Xpert MTB/RIF)}

Were the index test results interpreted without knowledge of the results Yes of the reference standard?

If a threshold was used, was it pre-specified? Yes

Could the conduct or interpretation of the index test have introduced Low risk
bias?

bias?

Are there concerns that the index test, its conduct, or interpretation

differ from the review question?

\section{DOMAIN 2: Index Test (Xpert Ultra)}

\section{DOMAIN 3: Reference Standard}

Is the reference standards likely to correctly classify the target condition? Yes

Were the reference standard results interpreted without knowledge of Yes

the results of the index tests?

For rifampicin resistance testing, were the reference standard results in-

terpreted without knowledge of the results of the index test?

\section{Could the reference standard, its conduct, or its interpretation have introduced bias?

\section{Are there concerns that the target condition as defined by the refer-} ence standard does not match the question?

\section{DOMAIN 4: Flow and Timing}

Was there an appropriate interval between index test and reference stan- Yes dard?

\begin{tabular}{lc}
\hline Did all patients receive the same reference standard? & Yes \\
\hline Were all patients included in the analysis? & Yes \\
\hline
\end{tabular}

\section{Could the patient flow have introduced bias?}

Low risk

Hillemann 2011

\section{Study characteristics}

$$
\text { Patient Sampling }
$$

Patient characteristics and setting
Cross-sectional, consecutive, prospective

Presenting signs and symptoms: patients with suspected $M$ tuberculosis or nontuberculous mycobacterial infection on the basis of clinical criteria 
Hillemann 2011 (Continued)

\author{
Age: not reported \\ Sex, female: not reported \\ Children: $5 \%$ \\ HIV infection: not reported \\ Clinical setting: national reference laboratory \\ Past history of TB: not reported \\ Patients on anti-TB treatment: not reported \\ Number of specimens evaluated: 200 \\ Laboratory level: central \\ Country: Germany \\ World Bank Income Classification: high income \\ High TB burden: no \\ High TB/HIV burden: no \\ High MDR-TB burden: no
}

\begin{tabular}{ll}
\hline Index tests & Xpert MTB/RIF \\
& $\begin{array}{l}\text { WHO SOP or manufacturer's protocol followed: yes } \\
\text { Manufacturer's involvement: yes, donation of index } \\
\text { test }\end{array}$ \\
\hline Target condition and reference standard(s) & $\begin{array}{l}\text { Target condition: pleural TB, TB meningitis, genitouri- } \\
\text { nary TB }\end{array}$ \\
$\begin{array}{l}\text { Reference standard for TB detection: } \mathrm{LJ} \text { and MGIT } \\
\text { Reference standard for rifampicin resistance: MGIT- } \\
\text { DST } \\
\text { Speciation: yes } \\
\text { Decontamination: } y \text { es, } \mathrm{NALC}-\mathrm{NaOH}\end{array}$
\end{tabular}

Flow and timing

Comparative

\title{
Notes
}

\section{Methodological quality}

\begin{tabular}{|c|c|c|c|}
\hline Item & $\begin{array}{l}\text { Authors' } \\
\text { judgement }\end{array}$ & Risk of bias & $\begin{array}{l}\text { Applicability } \\
\text { concerns }\end{array}$ \\
\hline
\end{tabular}

\section{DOMAIN 1: Patient Selection}

Was a consecutive or random sample of patients enrolled?

Was a case-control design avoided?
Yes

Yes 
Hillemann 2011 (Continued)

Did the study avoid inappropriate exclusions?

Yes

\section{Could the selection of patients have introduced bias?}

Low risk

Are there concerns that the included patients and setting do not match

Unclear the review question?

\section{DOMAIN 2: Index Test (Xpert MTB/RIF)}

Were the index test results interpreted without knowledge of the results of Yes the reference standard?

If a threshold was used, was it pre-specified? Yes

\section{Could the conduct or interpretation of the index test have introduced} bias?

Are there concerns that the index test, its conduct, or interpretation dif-

fer from the review question?

\section{DOMAIN 2: Index Test (Xpert Ultra)}

DOMAIN 3: Reference Standard

Is the reference standards likely to correctly classify the target condition? Unclear

Were the reference standard results interpreted without knowledge of the Yes results of the index tests?

For rifampicin resistance testing, were the reference standard results inter- Yes preted without knowledge of the results of the index test?

\section{Could the reference standard, its conduct, or its interpretation have in- \\ Unclear risk} troduced bias?

Are there concerns that the target condition as defined by the reference standard does not match the question?

\section{DOMAIN 4: Flow and Timing}

Was there an appropriate interval between index test and reference stan- $\quad$ Yes dard?

Did all patients receive the same reference standard?

Yes

Were all patients included in the analysis?

Yes

\section{Could the patient flow have introduced bias?}

Low risk

Iram 2015

\section{Study characteristics}


Iram 2015 (Continued)

Patient characteristics and setting
Presenting signs and symptoms: patients with clinical presentation, radiological findings, and histopathological evidence of extrapulmonary TB

Age: mean 37 years, range 10 to 80 years

Sex, female: $41 \%$

Children: $3 \%$

HIV infection: $2 \%$

Clinical setting: teaching hospital

Past history of TB: $53 \%$

Patients on anti-TB treatment: yes, $3 \%$

Number of specimens evaluated: 18

Laboratory level: intermediate

Country: Pakistan

World Bank Income Classification: middle income

High TB burden: yes

High TB/HIV burden: no

High MDR-TB burden: yes
Xpert MTB/RIF

WHO SOP or manufacturer's protocol followed: yes

Manufacturer's involvement: no

Target condition and reference standard(s)

Target condition: pleural TB, peritoneal TB

Reference standard for TB detection: LJ

Reference standard for rifampicin resistance: LJ-DST

Speciation: not reported

Decontamination: no

Flow and timing

Comparative

Notes

Methodological quality

\begin{tabular}{lll}
\hline Item & $\begin{array}{l}\text { Authors' } \\
\text { judgement }\end{array}$ & Risk of bias \\
\hline DOMAIN 1: Patient Selection & $\begin{array}{l}\text { Applicability } \\
\text { concerns }\end{array}$ \\
\hline Was a consecutive or random sample of patients enrolled? & Yes \\
\hline
\end{tabular}


Iram 2015 (Continued)

Was a case-control design avoided?

Did the study avoid inappropriate exclusions?

Could the selection of patients have introduced bias?

Low risk

Are there concerns that the included patients and setting do not match the review question?

\section{DOMAIN 2: Index Test (Xpert MTB/RIF)}

Were the index test results interpreted without knowledge of the results of Yes the reference standard?

If a threshold was used, was it pre-specified?

Yes

\section{Could the conduct or interpretation of the index test have introduced bias?

Are there concerns that the index test, its conduct, or interpretation dif-

fer from the review question?

DOMAIN 2: Index Test (Xpert Ultra)

\section{DOMAIN 3: Reference Standard}

Is the reference standards likely to correctly classify the target condition? Yes

Were the reference standard results interpreted without knowledge of the Yes results of the index tests?

For rifampicin resistance testing, were the reference standard results inter- Yes preted without knowledge of the results of the index test?

\section{Could the reference standard, its conduct, or its interpretation have in- Low risk troduced bias?}

Are there concerns that the target condition as defined by the reference standard does not match the question?

\section{DOMAIN 4: Flow and Timing}

Was there an appropriate interval between index test and reference stan- $\quad$ Yes dard?

Did all patients receive the same reference standard?

Were all patients included in the analysis?
Yes

Yes

\section{Could the patient flow have introduced bias?}

Low risk

\section{Study characteristics}


Kim 2015a (Continued)

Patient Sampling

Cross-sectional, consecutive, retrospective

Patient characteristics and setting

Presenting signs and symptoms: not reported

Age: median 59 years (IQR 44 to 71 years)

Sex, female: $47 \%$

Children: $7 \%$

HIV infection: $1 \%$

Clinical setting: tertiary care centre

Past history of TB: $9 \%$

Patients on anti-TB treatment: no

Number of specimens evaluated: 1209

Laboratory level: central

Country: Korea

World Bank Income Classification: high income

High TB burden: no

High TB/HIV burden: no

High MDR-TB burden: no

Index tests

Xpert MTB/RIF

WHO SOP or manufacturer's protocol followed: yes

Manufacturer's involvement: no

Target condition and reference standard(s)

Target condition: lymph node TB, pleural TB, TB meningitis, peritoneal TB, pericardial TB, bone and joint $T B$, genitourinary $T B$

Reference standard for TB detection: MGIT

Reference standard for rifampicin resistance: LJ-DST

Speciation: yes

Decontamination: yes, NALC-NaOH

Flow and timing

\section{Comparative}

\section{Notes}

\section{Methodological quality}

\begin{tabular}{|c|c|c|c|}
\hline Item & $\begin{array}{l}\text { Authors' } \\
\text { judgement }\end{array}$ & Risk of bias & $\begin{array}{l}\text { Applicability } \\
\text { concerns }\end{array}$ \\
\hline
\end{tabular}

DOMAIN 1: Patient Selection 
Kim 2015a (Continued)

Was a consecutive or random sample of patients enrolled?

Was a case-control design avoided? Yes

Did the study avoid inappropriate exclusions?

Yes

\section{Could the selection of patients have introduced bias?}

Are there concerns that the included patients and setting do not match the review question?

\section{DOMAIN 2: Index Test (Xpert MTB/RIF)}

Were the index test results interpreted without knowledge of the results of Yes the reference standard?

If a threshold was used, was it pre-specified? Yes

\section{Could the conduct or interpretation of the index test have introduced bias?

\section{DOMAIN 2: Index Test (Xpert Ultra)}

\section{DOMAIN 3: Reference Standard}

Is the reference standards likely to correctly classify the target condition? Unclear

Were the reference standard results interpreted without knowledge of the Yes results of the index tests?

For rifampicin resistance testing, were the reference standard results inter- Yes preted without knowledge of the results of the index test?

\section{Could the reference standard, its conduct, or its interpretation have in- troduced bias?

\section{Are there concerns that the target condition as defined by the reference} standard does not match the question?

\section{DOMAIN 4: Flow and Timing}

Was there an appropriate interval between index test and reference stan- Yes dard?

Did all patients receive the same reference standard?

Were all patients included in the analysis?
Yes

Yes

\section{Could the patient flow have introduced bias?}

Low risk 


\section{Study characteristics}

\section{Patient Sampling}

Patient characteristics and setting
Cross-sectional, manner of participant selection not reported, prospective

Presenting signs and symptoms: patients with suspected extrapulmonary TB

Age: mean 48 years (SD 10 years)

Sex, female: $39 \%$

Children: no

HIV infection: not reported

Clinical setting: tertiary care centre

Past history of TB: not reported

Patients on anti-TB treatment: no

Number of specimens evaluated: 414

Laboratory level: central

Country: China

World Bank Income Classification: middle income

High TB burden: yes

High TB/HIV burden: yes

High MDR-TB burden: yes

Index tests

Xpert MTB/RIF

WHO SOP or manufacturer's protocol followed: yes for pleural fluid, bone and joint TB fluid, urine, and peritoneal fluid; no for CSF

Manufacturer's involvement: no

Target condition: pleural TB, TB meningitis, peritoneal $T B$, bone and joint $T B$, genitourinary $T B$

Reference standard for TB detection: LJ

Reference standard for rifampicin resistance: LJ-DST

Speciation: yes

Decontamination: yes, NALC-NaOH

Flow and timing

Comparative

\section{Notes}

\section{Methodological quality}


Li 2017 (Continued)

$\begin{array}{lll}\text { Item } & \begin{array}{l}\text { Authors' judge- } \\ \text { ment }\end{array} & \begin{array}{c}\text { Risk of bias } \\ \text { Applicability } \\ \text { concerns }\end{array}\end{array}$

DOMAIN 1: Patient Selection

\begin{tabular}{lcc}
\hline Was a consecutive or random sample of patients enrolled? & Unclear \\
\hline Was a case-control design avoided? & Yes & Yes \\
\hline Did the study avoid inappropriate exclusions? & Unclear risk \\
\hline Could the selection of patients have introduced bias? & Unclear \\
\hline $\begin{array}{l}\text { Are there concerns that the included patients and setting do not } \\
\text { match the review question? }\end{array}$ & \\
\hline
\end{tabular}

\section{DOMAIN 2: Index Test (Xpert MTB/RIF)}

Were the index test results interpreted without knowledge of the results of Yes the reference standard?

\begin{tabular}{l}
\hline If a threshold was used, was it pre-specified? \\
$\begin{array}{l}\text { Could the conduct or interpretation of the index test have introduced } \\
\text { bias? }\end{array}$ \\
$\begin{array}{l}\text { Are there concerns that the index test, its conduct, or interpretation } \\
\text { differ from the review question? }\end{array}$
\end{tabular}

DOMAIN 2: Index Test (Xpert Ultra)

\section{DOMAIN 3: Reference Standard}

Is the reference standards likely to correctly classify the target condition? Unclear

Were the reference standard results interpreted without knowledge of the Yes results of the index tests?

For rifampicin resistance testing, were the reference standard results in- Yes terpreted without knowledge of the results of the index test?

Could the reference standard, its conduct, or its interpretation have Unclear risk
introduced bias?

Are there concerns that the target condition as defined by the reference standard does not match the question?

\section{DOMAIN 4: Flow and Timing}

Was there an appropriate interval between index test and reference stan- Yes dard?

\begin{tabular}{lc}
\hline Did all patients receive the same reference standard? & Yes \\
\hline Were all patients included in the analysis? & Yes
\end{tabular}




\section{Study characteristics}

Patient Sampling

Patient characteristics and setting
Cross-sectional, manner of patient selection not reported, retrospective

Presenting signs and symptoms: patients with suspected pleural TB based on standard clinical and radiological criteria, including a persistent cough of 2 weeks or more, unexplained fever for 2 weeks or more, weight loss, and radiological evidence of pleural effusion

Age: mainly adult; $12 \%<25$ years

Sex, female: $22 \%$

Children: not reported

HIV infection: not reported

Clinical setting: national TB referral hospital

Past history of TB: not reported

Participants on anti-TB treatment: not reported

Number of specimens evaluated: 219

Laboratory level: central

Country: China

World Bank Income Classification: middle income

High TB burden: yes

High TB/HIV burden: yes

High MDR-TB burden: yes

Xpert MTB/RIF

WHO SOP or manufacturer's protocol followed: yes

Target condition and reference standard(s)

Pleural TB

CRS: clinically diagnosed and microbiologically confirmed

Comparative

Notes

\section{Methodological quality}

\section{Item}

Authors'

Risk of bias

judgement

Applicability concerns 
Liang 2019 (Continued)

DOMAIN 1: Patient Selection

\begin{tabular}{ll}
\hline Was a consecutive or random sample of patients enrolled? & Unclear \\
\hline Was a case-control design avoided? & Yes \\
\hline Did the study avoid inappropriate exclusions? & Yes \\
\hline Could the selection of patients have introduced bias? & Unclear risk \\
\hline $\begin{array}{l}\text { Are there concerns that the included patients and setting do not match } \\
\text { the review question? }\end{array}$
\end{tabular}

\title{
DOMAIN 2: Index Test (Xpert MTB/RIF)
}

Were the index test results interpreted without knowledge of the results of Yes the reference standard?

If a threshold was used, was it pre-specified? Yes

\author{
Could the conduct or interpretation of the index test have introduced \\ bias?

Are there concerns that the index test, its conduct, or interpretation differ from the review question?

DOMAIN 2: Index Test (Xpert Ultra)

\section{DOMAIN 3: Reference Standard}

Is the reference standards likely to correctly classify the target condition? Yes

Were the reference standard results interpreted without knowledge of the Yes results of the index tests?

For rifampicin resistance testing, were the reference standard results inter- Unclear preted without knowledge of the results of the index test?

\section{Could the reference standard, its conduct, or its interpretation have in- Low risk troduced bias?}

Are there concerns that the target condition as defined by the reference standard does not match the question?

\section{DOMAIN 4: Flow and Timing}

Was there an appropriate interval between index test and reference stan- Yes dard?

Did all patients receive the same reference standard?

Were all patients included in the analysis?
Yes

Yes 
Ligthelm 2011

\section{Study characteristics}

\section{Patient Sampling}

Patient characteristics and setting
Cross-sectional, consecutive, prospective

Presenting signs and symptoms: patients with suspicion of lymph node TB

Age: < 5 years $4 \% ; 5$ to 20 years $13 \%$; > 20 years $83 \%$

Sex, female: $58 \%$

Children: estimated $<15 \%$

HIV infection: $19 \%$

Clinical setting: university hospital (inpatient and outpatient)

Past history of TB: not reported

Patients on anti-TB treatment: not reported

Number of specimens evaluated: 48

Laboratory level: central

Country: South Africa

World Bank Income Classification: middle income

High TB burden: yes

High TB/HIV burden: yes

High MDR-TB burden: yes

Index tests

Xpert MTB/RIF

WHO SOP or manufacturer's protocol followed: yes

Manufacturer's involvement: no

Target condition and reference standard(s)
Target condition: lymph node TB

Reference standard for TB detection: MGIT

Reference standard for rifampicin resistance: MTBDRplus

Speciation: yes

Decontamination: no

\section{Flow and timing}

"It is unlikely that our patient cohort had exacerbated disease compared to patients presenting at primary health care clinics, as these patients are routinely referred from the primary health care clinic to the referral centre for FNAB (fine needle aspiration biopsy)" 
Ligthelm 2011 (Continued)

Methodological quality

\begin{tabular}{lll}
\hline Item & $\begin{array}{l}\text { Authors' judge- } \\
\text { ment }\end{array}$ & $\begin{array}{c}\text { Risk of bias } \\
\text { Applicability } \\
\text { concerns }\end{array}$ \\
\hline
\end{tabular}

DOMAIN 1: Patient Selection

\begin{tabular}{lcc}
\hline Was a consecutive or random sample of patients enrolled? & Yes & Yes \\
\hline Was a case-control design avoided? & Yes & Low risk \\
\hline Did the study avoid inappropriate exclusions? & \\
\hline Could the selection of patients have introduced bias? & Unclear \\
\hline $\begin{array}{l}\text { Are there concerns that the included patients and setting do not } \\
\text { match the review question? }\end{array}$
\end{tabular}

\section{DOMAIN 2: Index Test (Xpert MTB/RIF)}

Were the index test results interpreted without knowledge of the results Yes of the reference standard?

If a threshold was used, was it pre-specified? Yes

\section{Could the conduct or interpretation of the index test have intro- \\ Low risk} duced bias?

\section{Are there concerns that the index test, its conduct, or interpretation} differ from the review question?

\section{DOMAIN 2: Index Test (Xpert Ultra)}

\section{DOMAIN 3: Reference Standard}

Is the reference standards likely to correctly classify the target condi- Yes tion?

Were the reference standard results interpreted without knowledge of Yes the results of the index tests?

For rifampicin resistance testing, were the reference standard results Yes interpreted without knowledge of the results of the index test?

\section{Could the reference standard, its conduct, or its interpretation have introduced bias?}

\section{Are there concerns that the target condition as defined by the refer-} ence standard does not match the question?

\section{DOMAIN 4: Flow and Timing}

Was there an appropriate interval between index test and reference Yes standard? 
Ligthelm 2011 (Continued)

Were all patients included in the analysis?

Yes

Could the patient flow have introduced bias?

Low risk

\section{Lusiba 2014}

\section{Study characteristics}

\begin{tabular}{ll} 
Patient Sampling $\quad$ Cross-sectional, consecutive, prospective \\
\hline
\end{tabular}

Patient characteristics and setting

Presenting signs and symptoms: patients with suspected pleural TB based on clinical signs and symptoms and radiological evidence of a pleural effusion that was considered large enough for a pleural biopsy

Age: mean 34 years, SD 13 years

Sex, female: $43 \%$

Children: no

HIV infection: $45 \%$

Clinical setting: tertiary care centre (inpatient and outpatient)

Past history of TB: not reported

Patients on anti-TB treatment: no

Number of specimens evaluated: 116

Laboratory level: central

Country: Uganda

World Bank Income Classification: low income

High TB burden: no

High TB/HIV burden: yes

High MDR-TB burden: no

WHO SOP or manufacturer's protocol followed: no

Manufacturer's involvement: no

Speciation: not reported

Decontamination: no 
Lusiba 2014 (Continued)

Flow and timing

\section{Comparative}

Notes

\section{Methodological quality}

\begin{tabular}{|c|c|c|c|}
\hline Item & $\begin{array}{l}\text { Authors' } \\
\text { judgement }\end{array}$ & Risk of bias & $\begin{array}{l}\text { Applicability } \\
\text { concerns }\end{array}$ \\
\hline
\end{tabular}

DOMAIN 1: Patient Selection

Was a consecutive or random sample of patients enrolled? Yes

Was a case-control design avoided? Yes

Did the study avoid inappropriate exclusions? Yes

\begin{tabular}{ll}
\hline Could the selection of patients have introduced bias? Low risk
\end{tabular}

Are there concerns that the included patients and setting do not match
the review question?

\section{DOMAIN 2: Index Test (Xpert MTB/RIF)}

Were the index test results interpreted without knowledge of the results of Yes the reference standard?

\begin{tabular}{ll}
\hline If a threshold was used, was it pre-specified? & Yes
\end{tabular}

Could the conduct or interpretation of the index test have introduced Low risk
bias?

Could the conduct or interpretation of the index test have introduced Low risk

Are there concerns that the index test, its conduct, or interpretation differ from the review question?

DOMAIN 2: Index Test (Xpert Ultra)

\section{DOMAIN 3: Reference Standard}

Is the reference standards likely to correctly classify the target condition? Yes
$\begin{aligned} & \text { Were the reference standard results interpreted without knowledge of the Unclear } \\ & \text { results of the index tests? }\end{aligned}$

For rifampicin resistance testing, were the reference standard results inter- Yes preted without knowledge of the results of the index test?

Could the reference standard, its conduct, or its interpretation have in-
troduced bias?

\section{Are there concerns that the target condition as defined by the reference} standard does not match the question?

\section{DOMAIN 4: Flow and Timing}


Lusiba 2014 (Continued)

Was there an appropriate interval between index test and reference stan- Yes dard?

Did all patients receive the same reference standard? Yes

Were all patients included in the analysis? Yes

Malbruny 2011

\section{Study characteristics}

\section{Patient Sampling}

Cross-sectional, manner of participant selection by convenience, prospective

\section{Patient characteristics and setting}

Presenting signs and symptoms: patients with clinical suspicion of TB

Age: median 52 years

Sex, female: $40 \%$

Children: $7 \%$

HIV infection: not reported

Clinical setting: university hospital

Past history of TB: not reported

Patients on anti-TB treatment: not reported

Number of specimens evaluated: 67

Laboratory level: central

Country: France

World Bank Income Classification: high income

High TB burden: no

High TB/HIV burden: no

High MDR-TB burden: no

WHO SOP or manufacturer's protocol followed: no

Manufacturer's involvement: no joint TB, peritoneal TB, genitourinary TB

Reference standard for TB detection: MGIT and Coletsos slants

Reference standard for rifampicin resistance: MGITDST 
Malbruny 2011 (Continued)

Speciation: yes

Decontamination: no

Flow and timing

Comparative

Notes

Methodological quality

\begin{tabular}{|c|c|c|c|}
\hline Item & $\begin{array}{l}\text { Authors' } \\
\text { judgement }\end{array}$ & Risk of bias & $\begin{array}{l}\text { Applicability } \\
\text { concerns }\end{array}$ \\
\hline
\end{tabular}

DOMAIN 1: Patient Selection

Was a consecutive or random sample of patients enrolled?

Was a case-control design avoided?

Yes

Did the study avoid inappropriate exclusions?

Yes

Could the selection of patients have introduced bias?

High risk

Are there concerns that the included patients and setting do not match the review question?

Unclear

\section{DOMAIN 2: Index Test (Xpert MTB/RIF)}

Were the index test results interpreted without knowledge of the results of Yes the reference standard?

If a threshold was used, was it pre-specified?

Yes

\section{Could the conduct or interpretation of the index test have introduced bias?}

Are there concerns that the index test, its conduct, or interpretation differ from the review question?

\section{DOMAIN 2: Index Test (Xpert Ultra)}

\section{DOMAIN 3: Reference Standard}

Is the reference standards likely to correctly classify the target condition? Yes

Were the reference standard results interpreted without knowledge of the Yes results of the index tests?

For rifampicin resistance testing, were the reference standard results inter- Yes preted without knowledge of the results of the index test?

Could the reference standard, its conduct, or its interpretation have introduced bias? 
Malbruny 2011 (Continued)

\section{Are there concerns that the target condition as defined by the reference standard does not match the question?}

\section{DOMAIN 4: Flow and Timing}

Was there an appropriate interval between index test and reference stan-

Yes dard?

Did all patients receive the same reference standard?

Yes

Were all patients included in the analysis?

Yes

Could the patient flow have introduced bias?

Low risk

Meldau 2014

\section{Study characteristics}

Patient Sampling

Patient characteristics and setting
Cross-sectional, consecutive, prospective

Presenting signs and symptoms: patients presumed to have pleural TB with any symptoms, including cough, fever, night sweats, loss of weight, haemoptysis, and chest pain, along with features consistent with a pleural effusion on chest X-ray

Age: definitive TB: median 39 years (IQR 29 to 55 years); non-TB: median 61 years (IQR 54 to 69 years)

Sex, female: $40 \%$

Children: no

HIV infection: $15 \%$

Clinical setting: tertiary care hospital

Past history of TB: $13 \%$

Patients on anti-TB treatment: no

Number of specimens evaluated against culture: 76

Number of specimens evaluated against a composite reference standard: 88

Laboratory level: central

Country: South Africa

World Bank Income Classification: middle income

High TB burden: yes

High TB/HIV burden: yes

High MDR-TB burden: yes 
Manufacturer's involvement: no

Reference standard for TB detection: MGIT

Reference standard for rifampicin resistance: MGIT-DST

Speciation: yes

Decontamination: no

\section{Flow and timing}

\section{Comparative}

$$
\text { Notes }
$$

\section{Methodological quality}

\begin{tabular}{lll}
\hline Item & $\begin{array}{l}\text { Authors' judge- } \\
\text { ment }\end{array}$ & $\begin{array}{l}\text { Risk of bias } \\
\text { Applicability } \\
\text { concerns }\end{array}$ \\
\hline
\end{tabular}

DOMAIN 1: Patient Selection

\begin{tabular}{lcc}
\hline Was a consecutive or random sample of patients enrolled? & Yes & Yes \\
\hline Was a case-control design avoided? & Yes & Low risk \\
\hline Did the study avoid inappropriate exclusions? & \\
\hline Could the selection of patients have introduced bias? & Unclear \\
\hline $\begin{array}{l}\text { Are there concerns that the included patients and setting do not } \\
\text { match the review question? }\end{array}$
\end{tabular}

\section{DOMAIN 2: Index Test (Xpert MTB/RIF)}

Were the index test results interpreted without knowledge of the results Yes of the reference standard?

If a threshold was used, was it pre-specified? Yes

Could the conduct or interpretation of the index test have intro- Low risk
duced bias?
Are there concerns that the index test, its conduct, or interpretation differ from the review question?

DOMAIN 2: Index Test (Xpert Ultra)

\section{DOMAIN 3: Reference Standard}

Is the reference standards likely to correctly classify the target condi- $\quad$ Yes tion? 
Meldau 2014 (Continued)

Were the reference standard results interpreted without knowledge of Yes the results of the index tests?

For rifampicin resistance testing, were the reference standard results Yes interpreted without knowledge of the results of the index test?

\section{Could the reference standard, its conduct, or its interpretation have introduced bias? \\ Low risk}

Are there concerns that the target condition as defined by the reference standard does not match the question?

\section{DOMAIN 4: Flow and Timing}

Was there an appropriate interval between index test and reference Yes standard?

\begin{tabular}{lcl}
\hline Did all patients receive the same reference standard? & Yes & Yes \\
\hline Were all patients included in the analysis? & Low risk \\
\hline Could the patient flow have introduced bias? & \\
\hline
\end{tabular}

Meldau 2019

\section{Study characteristics}

\section{Patient Sampling}

Patient characteristics and setting
Cohort, consecutive, prospective

Presenting signs and symptoms: patients with any TB symptoms including any cough, fever, night sweats, loss of weight, haemoptysis or chest pain or both, and features consistent with a pleural effusion on chest $x$ ray

Age: Adult; median 39 years (IQR 28 to 57 )

Sex, female: $11 \%$

Children: not reported

HIV infection: definite TB: $7 \%$

Clinical setting: tertiary care hospital

Past history of TB: $6 \%$

Participants on anti-TB treatment: not reported

Number of specimens evaluated: 149

Laboratory level: central

Country: South Africa

World Bank Income Classification: middle

High TB burden: yes 
Meldau 2019 (Continued)

High TB/HIV burden: yes

High MDR-TB burden: yes

Index tests

Xpert MTB/RIF and Xpert Ultra

WHO SOP or manufacturer's protocol followed: yes

Target condition and reference standard(s)

Pleural tuberculosis

Composite reference standard: MGIT culture and/or histology

\begin{tabular}{llll}
\hline Flow and timing & \\
\hline Comparative & & \\
\hline Notes & Authors' & Risk of bias & Applicability \\
Methodological quality & judgement & concerns
\end{tabular}

\section{DOMAIN 1: Patient Selection}

Was a consecutive or random sample of patients enrolled? Yes

\begin{tabular}{ll}
\hline Was a case-control design avoided? & Yes \\
\hline
\end{tabular}

Did the study avoid inappropriate exclusions? Yes

\begin{tabular}{ll}
\hline Could the selection of patients have introduced bias? & Low risk
\end{tabular}

Are there concerns that the included patients and setting do not match Unclear
the review question?

\section{DOMAIN 2: Index Test (Xpert MTB/RIF)}

Were the index test results interpreted without knowledge of the results of Yes the reference standard?

If a threshold was used, was it pre-specified? Yes

Could the conduct or interpretation of the index test have introduced L L risk
bias?

bias?

Are there concerns that the index test, its conduct, or interpretation differ from the review question?

\section{DOMAIN 2: Index Test (Xpert Ultra)}

Were the index test results interpreted without knowledge of the results of Yes the reference standard?

If a threshold was used, was it pre-specified?

Yes 
Meldau 2019 (Continued)

Could the conduct or interpretation of the index test have introduced

Low risk bias?

Are there concerns that the index test, its conduct, or interpretation dif-

Low concern fer from the review question?

\section{DOMAIN 3: Reference Standard}

Is the reference standards likely to correctly classify the target condition? Yes

Were the reference standard results interpreted without knowledge of the Yes results of the index tests?

For rifampicin resistance testing, were the reference standard results interpreted without knowledge of the results of the index test?

\section{Could the reference standard, its conduct, or its interpretation have in- troduced bias?

\section{DOMAIN 4: Flow and Timing}

Was there an appropriate interval between index test and reference stan- Yes dard?

Did all patients receive the same reference standard? Yes

Were all patients included in the analysis?

Yes

\section{Could the patient flow have introduced bias?}

Low risk

Metcalf 2018

\section{Study characteristics}

Patient Sampling

Patient characteristics and setting
Cross-sectional, consecutive, prospective

Presenting signs and symptoms: patients presenting with a suspected diagnosis of TB meningitis

Age: 18 and older

Sex, female: $27 \%$

Children: No

HIV infection: $62 \%$

Clinical setting: inpatient

Past history of TB: $30 \%$

Participants on anti-TB treatment: no

Number of specimens evaluated: 37 
Metcalf 2018 (Continued)

\author{
Laboratory level: central \\ Country: Peru \\ World Bank Income Classification: middle income \\ High TB burden: no \\ High TB/HIV burden: no \\ High MDR-TB burden: yes
}

\begin{tabular}{ll}
\hline Index tests & Xpert MTB/RIF \\
& WHO SOP or manufacturer's protocol followed: yes \\
\hline Target condition and reference standard(s) & TB meningitis \\
& MGIT, Ogawa \\
& Speciation: not reported \\
\hline Flow and timing & \\
\hline Comparative & \\
\hline Notes & Risk of bias \\
\hline Methodological quality & $\begin{array}{l}\text { Applicability } \\
\text { concerns }\end{array}$ \\
\hline Item & judgement \\
\hline
\end{tabular}

\title{
DOMAIN 1: Patient Selection
}

Was a consecutive or random sample of patients enrolled? Yes

\begin{tabular}{lcc}
\hline Was a case-control design avoided? & Yes & Yes \\
\hline Did the study avoid inappropriate exclusions? & Low risk \\
\hline Could the selection of patients have introduced bias? & Low concern \\
\hline $\begin{array}{l}\text { Are there concerns that the included patients and setting do not match } \\
\text { the review question? }\end{array}$
\end{tabular}

\section{DOMAIN 2: Index Test (Xpert MTB/RIF)}

Were the index test results interpreted without knowledge of the results of Yes the reference standard?

If a threshold was used, was it pre-specified? Yes

\begin{tabular}{|c|c|}
\hline $\begin{array}{l}\text { Could the conduct or interpretation of the index test have introduced } \\
\text { bias? }\end{array}$ & Low risk \\
\hline
\end{tabular}

Are there concerns that the index test, its conduct, or interpretation differ from the review question? 
Metcalf 2018 (Continued)

DOMAIN 2: Index Test (Xpert Ultra)

\section{DOMAIN 3: Reference Standard}

Is the reference standards likely to correctly classify the target condition? Yes

Were the reference standard results interpreted without knowledge of the Unclear results of the index tests?

For rifampicin resistance testing, were the reference standard results inter- Unclear preted without knowledge of the results of the index test?
Could the reference standard, its conduct, or its interpretation have in- troduced bias?
Unclear risk
Are there concerns that the target condition as defined by the reference standard does not match the question?

\section{DOMAIN 4: Flow and Timing}

Was there an appropriate interval between index test and reference stan- Yes dard?

Did all patients receive the same reference standard? Yes

Were all patients included in the analysis? Yes

Could the patient flow have introduced bias?

Low risk

Nataraj 2016

\section{Study characteristics}

\begin{tabular}{ll}
\hline Patient Sampling & Cross-sectional, consecutive, prospective \\
\hline Patient characteristics and setting & $\begin{array}{l}\text { Presenting signs and symptoms: patients with clinical suspicion } \\
\text { of extrapulmonary TB }\end{array}$ \\
$\begin{array}{l}\text { Age: }<14 \text { years } 13 \% ; 15 \text { to } 45 \text { years } 52 \% ;>45 \text { years } 34 \% ; \text { range } 2 \\
\text { months to } 78 \text { years }\end{array}$ & $\begin{array}{l}\text { Sex, female: } 44 \% \\
\text { Children: } 13 \%\end{array}$ \\
& $\begin{array}{l}\text { HIV infection: not reported } \\
\text { Clinical setting: tertiary care centre (inpatient and outpatient) }\end{array}$ \\
Past history of TB: not reported \\
Patients on anti-TB treatment: not reported \\
Number of specimens evaluated: 494 \\
Laboratory level: intermediate \\
Country: India
\end{tabular}


High TB burden: yes

High TB/HIV burden: yes

High MDR-TB burden: yes

WHO SOP or manufacturer's protocol followed: yes

Manufacturer's involvement: no

Target condition and reference standard(s)

Target condition: pleural TB, lymph node TB, TB meningitis, bone and joint TB, genitourinary TB

Reference standard TB detection: LJ

Reference standard rifampicin resistance detection: LJ-DST

Speciation: yes

Decontamination: yes, NALC-NaOH

Flow and timing

Comparative

Notes Patients on treatment may have been included, although the number was not reported: "Of the two specimens that were smear-positive and smear-negative on both culture and Xpert, one was pleural fluid from a patient who had been receiving Category II anti-tuberculosis treatment for 2 months and the other was pus aspirated from an axillary lymph node"

\section{Methodological quality}

\begin{tabular}{|c|c|c|c|}
\hline Item & $\begin{array}{l}\text { Authors' judge- } \\
\text { ment }\end{array}$ & Risk of bias & $\begin{array}{l}\text { Applicability con- } \\
\text { cerns }\end{array}$ \\
\hline
\end{tabular}

DOMAIN 1: Patient Selection

\begin{tabular}{lll}
\hline Was a consecutive or random sample of patients enrolled? & Yes \\
\hline Was a case-control design avoided? & Yes & Yes \\
\hline Did the study avoid inappropriate exclusions? & Low risk \\
\hline Could the selection of patients have introduced bias? & \\
\hline $\begin{array}{l}\text { Are there concerns that the included patients and setting do } \\
\text { not match the review question? }\end{array}$ & \\
\hline
\end{tabular}

\section{DOMAIN 2: Index Test (Xpert MTB/RIF)}

Were the index test results interpreted without knowledge of the Yes results of the reference standard? 
Nataraj 2016 (Continued)

Could the conduct or interpretation of the index test have in-

troduced bias?

Are there concerns that the index test, its conduct, or inter-

Low concern pretation differ from the review question?

\section{DOMAIN 2: Index Test (Xpert Ultra)}

\section{DOMAIN 3: Reference Standard}

\section{Is the reference standards likely to correctly classify the target Unclear} condition?

Were the reference standard results interpreted without knowl- Yes edge of the results of the index tests?

For rifampicin resistance testing, were the reference standard re- Yes sults interpreted without knowledge of the results of the index test?

Could the reference standard, its conduct, or its interpreta- Unclear risk
tion have introduced bias?
tion have introduced bias?

\section{Are there concerns that the target condition as defined by the} reference standard does not match the question?

\section{DOMAIN 4: Flow and Timing}

Was there an appropriate interval between index test and refer- Yes ence standard?

\begin{tabular}{ll}
\hline Did all patients receive the same reference standard? & Yes \\
\hline Were all patients included in the analysis? & Yes \\
\hline
\end{tabular}

\section{Could the patient flow have introduced bias?}

Low risk

Nhu 2014

\section{Study characteristics}

\begin{tabular}{|c|c|}
\hline Patient Sampling & Cross-sectional, consecutive, prospective \\
\hline \multirow[t]{6}{*}{ Patient characteristics and setting } & $\begin{array}{l}\text { Presenting signs and symptoms: patients suspected } \\
\text { of having TB meningitis with at least } 5 \text { days of menin- } \\
\text { gitis symptoms, nuchal rigidity, and CSF abnormali- } \\
\text { ties }\end{array}$ \\
\hline & Age: $>18$ years \\
\hline & Sex, female: not reported \\
\hline & Children: no \\
\hline & HIV infection: $21 \%$ \\
\hline & Clinical setting: university hospital \\
\hline
\end{tabular}


Nhu 2014 (Continued)

Past history of TB: not reported

Patients on anti-TB treatment: no

Number of specimens evaluated: 379

Laboratory level: central

Country: Vietnam

World Bank Income Classification: middle income

High TB burden: yes

High TB/HIV burden: no

High MDR-TB burden: yes

\begin{tabular}{ll}
\hline Index tests & Xpert MTB/RIF \\
& $\begin{array}{l}\text { WHO SOP or manufacturer's protocol followed: no } \\
\text { Manufacturer's involvement: yes, donation of index } \\
\text { test }\end{array}$ \\
\hline Target condition and reference standard(s) & $\begin{array}{l}\text { Target condition: TB meningitis } \\
\text { Reference standard TB detection: MGIT }\end{array}$ \\
$\begin{array}{l}\text { Reference standard rifampicin resistance detection: } \\
\text { MGIT-DST and MTBDRplus } \\
\text { Speciation: yes }\end{array}$ \\
Decontamination: no
\end{tabular}

Flow and timing

Comparative

Analysis by uniform case definition also included

\section{Methodological quality}

\begin{tabular}{|c|c|c|c|}
\hline Item & $\begin{array}{l}\text { Authors' } \\
\text { judgement }\end{array}$ & Risk of bias & $\begin{array}{l}\text { Applicability } \\
\text { concerns }\end{array}$ \\
\hline
\end{tabular}

DOMAIN 1: Patient Selection

\begin{tabular}{lcc}
\hline Was a consecutive or random sample of patients enrolled? & Yes \\
\hline Was a case-control design avoided? & Yes & Yes \\
\hline Did the study avoid inappropriate exclusions? & Low risk \\
\hline Could the selection of patients have introduced bias? & Low concern \\
\hline $\begin{array}{l}\text { Are there concerns that the included patients and setting do not match } \\
\text { the review question? }\end{array}$
\end{tabular}

\section{DOMAIN 2: Index Test (Xpert MTB/RIF)}


Nhu 2014 (Continued)

Were the index test results interpreted without knowledge of the results of Yes the reference standard?

If a threshold was used, was it pre-specified?

Yes

Could the conduct or interpretation of the index test have introduced bias?

Are there concerns that the index test, its conduct, or interpretation dif-

fer from the review question?

DOMAIN 2: Index Test (Xpert Ultra)

\section{DOMAIN 3: Reference Standard}

Is the reference standards likely to correctly classify the target condition? Yes

Were the reference standard results interpreted without knowledge of the Yes results of the index tests?

For rifampicin resistance testing, were the reference standard results inter- Yes preted without knowledge of the results of the index test?

Could the reference standard, its conduct, or its interpretation have in- Low risk troduced bias?

Are there concerns that the target condition as defined by the reference standard does not match the question?

\section{DOMAIN 4: Flow and Timing}

Was there an appropriate interval between index test and reference stan- Yes dard?

Did all patients receive the same reference standard? Yes

Were all patients included in the analysis?

Yes

\section{Ozkutuk 2014}

\section{Study characteristics}

Patient Sampling Cross-sectional, consecutive, prospective

Patient characteristics and setting

Presenting signs and symptoms: not reported

Age: median 54 years, range 1 to 99 years

Sex, female: $47 \%$

Children: $3 \%$

HIV infection: not reported 
Clinical setting: tertiary care centre (inpatient and outpatient)

Past history of TB: not reported

Patients on anti-TB treatment: not reported

Number of specimens evaluated: 1022

Laboratory level: central

Country: Turkey

World Bank Income Classification: middle

High TB burden: no

High TB/HIV burden: no

High MDR-TB burden: no

\begin{tabular}{ll}
\hline Index tests & Xpert MTB/RIF \\
& WHO SOP or manufacturer's protocol followed: yes \\
& Manufacturer's involvement: no \\
\hline Target condition and reference standard(s) & $\begin{array}{l}\text { Target condition: pleural TB, lymph node TB, TB } \\
\text { meningitis, genitourinary TB, bone and joint TB, peri- } \\
\text { cardial TB, peritoneal TB } \\
\text { Reference standard TB detection: LJ and MGIT }\end{array}$ \\
Reference standard rifampicin resistance detection: \\
MGIT-DST \\
Speciation: yes \\
Decontamination: no
\end{tabular}

Flow and timing

Comparative

Notes

\section{Methodological quality}

\begin{tabular}{|c|c|c|c|}
\hline Item & $\begin{array}{l}\text { Authors' } \\
\text { judgement }\end{array}$ & Risk of bias & $\begin{array}{l}\text { Applicability } \\
\text { concerns }\end{array}$ \\
\hline
\end{tabular}

DOMAIN 1: Patient Selection

Was a consecutive or random sample of patients enrolled? Yes

Was a case-control design avoided? Yes

Did the study avoid inappropriate exclusions? Yes

Could the selection of patients have introduced bias?

Low risk 
Ozkutuk 2014 (Continued) Are there concerns that the included patients and setting do not match
the review question?

\section{DOMAIN 2: Index Test (Xpert MTB/RIF)}

Were the index test results interpreted without knowledge of the results of Yes the reference standard?

If a threshold was used, was it pre-specified? Yes

\section{Could the conduct or interpretation of the index test have introduced bias?

fer from the review question?

\section{DOMAIN 2: Index Test (Xpert Ultra)}

\section{DOMAIN 3: Reference Standard}

Is the reference standards likely to correctly classify the target condition? Yes

Were the reference standard results interpreted without knowledge of the Yes results of the index tests?

For rifampicin resistance testing, were the reference standard results inter- Yes preted without knowledge of the results of the index test?

\section{Could the reference standard, its conduct, or its interpretation have in- Low risk troduced bias?}

\section{Are there concerns that the target condition as defined by the reference standard does not match the question?}

\section{DOMAIN 4: Flow and Timing}

Was there an appropriate interval between index test and reference stan- Yes dard?

\begin{tabular}{lc}
\hline Did all patients receive the same reference standard? & Yes \\
\hline Were all patients included in the analysis? & Yes
\end{tabular}

\section{Could the patient flow have introduced bias?}

Low risk

\section{Pandie 2014}

\section{Study characteristics}

$$
\text { Patient Sampling }
$$

Patient characteristics and setting
Cross-sectional, consecutive, prospective

Presenting signs and symptoms: patients with presence of a large pericardial effusion amenable to safe pericardiocentesis (> $10 \mathrm{~mm}$ echo-free space around the heart in diastole) 
Pandie 2014 (Continued)

Age: median 34 years (IQR 29 to 42)

Sex, female: $38 \%$

Children: no

HIV infection: $74 \%$

Clinical setting: 4 district hospitals and 1 tertiary cen-

tre (inpatient)

Past history of TB: not reported

Patients on anti-TB treatment: no

Number of specimens evaluated: 134

Laboratory level: central

Country: South Africa

World Bank Income Classification: middle income

High TB burden: yes

High TB/HIV burden: yes

High MDR-TB burden: yes

WHO SOP or manufacturer's protocol followed: yes

Manufacturer's involvement: no

Target condition and reference standard(s)

Target condition: pericardial TB

Reference standard TB detection: MGIT

Reference standard rifampicin resistance detection: MTBDRplus

Speciation: yes

Decontamination: no

\section{Flow and timing}

Comparative

Notes

\section{Methodological quality}

\begin{tabular}{|c|c|c|c|}
\hline Item & $\begin{array}{l}\text { Authors' } \\
\text { judgement }\end{array}$ & Risk of bias & $\begin{array}{l}\text { Applicability } \\
\text { concerns }\end{array}$ \\
\hline
\end{tabular}

\section{DOMAIN 1: Patient Selection}

Was a consecutive or random sample of patients enrolled?

Yes

\begin{tabular}{ll}
\hline Was a case-control design avoided? & Yes \\
\hline Did the study avoid inappropriate exclusions? & Yes \\
\hline
\end{tabular}


Pandie 2014 (Continued)

Could the selection of patients have introduced bias?

Low risk

Are there concerns that the included patients and setting do not match

Low concern the review question?

\section{DOMAIN 2: Index Test (Xpert MTB/RIF)}

Were the index test results interpreted without knowledge of the results of Yes the reference standard?

If a threshold was used, was it pre-specified?

Yes

\section{Could the conduct or interpretation of the index test have introduced bias?

Are there concerns that the index test, its conduct, or interpretation dif-

fer from the review question?

\section{DOMAIN 2: Index Test (Xpert Ultra)}

\section{DOMAIN 3: Reference Standard}

Is the reference standards likely to correctly classify the target condition? Yes

Were the reference standard results interpreted without knowledge of the Yes results of the index tests?

For rifampicin resistance testing, were the reference standard results inter- Yes preted without knowledge of the results of the index test?

\section{Could the reference standard, its conduct, or its interpretation have in- Low risk troduced bias?}

Are there concerns that the target condition as defined by the reference standard does not match the question?

DOMAIN 4: Flow and Timing

Was there an appropriate interval between index test and reference stan- Yes dard?

Did all patients receive the same reference standard? Yes

Were all patients included in the analysis? Yes

Could the patient flow have introduced bias?

Low risk

\section{Patel 2013}

\section{Study characteristics}

\begin{tabular}{ll}
\hline Patient Sampling & Cross-sectional, consecutive, prospective \\
\hline Patient characteristics and setting & $\begin{array}{l}\text { Presenting signs and symptoms: patients with clinical } \\
\text { suspicion of meningitis }\end{array}$
\end{tabular}


Patel 2013 (Continued)

Age: mean 33 years (SD 9)

Sex, female: $61 \%$

Children: $2 \%$

HIV infection: $87 \%$

Clinical setting: tertiary care centre (inpatient and outpatient)

Past history of TB: $31 \%$

Patients on anti-TB treatment: no

Number of specimens evaluated: 59

Laboratory level: central

Country: South Africa

World Bank Income Classification: middle income

High TB burden: yes

High TB/HIV burden: yes

High MDR-TB burden: yes

WHO SOP or manufacturer's protocol followed: yes

Manufacturer's involvement: no

Target condition and reference standard(s)

Target condition: TB meningitis

Reference standard TB detection: MGIT

Reference standard rifampicin resistance detection: MGIT-DST

Speciation: yes

Decontamination: no

\section{Flow and timing}

Comparative

\begin{tabular}{|c|c|c|c|}
\hline Notes & \multicolumn{3}{|c|}{ Study used frozen specimens } \\
\hline \multicolumn{4}{|c|}{ Methodological quality } \\
\hline Item & $\begin{array}{l}\text { Authors' } \\
\text { judgement }\end{array}$ & Risk of bias & $\begin{array}{l}\text { Applicability } \\
\text { concerns }\end{array}$ \\
\hline
\end{tabular}

\section{DOMAIN 1: Patient Selection}

\begin{tabular}{ll} 
Was a consecutive or random sample of patients enrolled? & Yes \\
\hline Was a case-control design avoided? & Yes \\
\hline Did the study avoid inappropriate exclusions? & Yes \\
\hline
\end{tabular}


Patel 2013 (Continued)

Could the selection of patients have introduced bias?

Low risk

Are there concerns that the included patients and setting do not match

Low concern the review question?

\section{DOMAIN 2: Index Test (Xpert MTB/RIF)}

Were the index test results interpreted without knowledge of the results of Yes the reference standard?

If a threshold was used, was it pre-specified?

Yes

\section{Could the conduct or interpretation of the index test have introduced bias?

Are there concerns that the index test, its conduct, or interpretation dif-

fer from the review question?

DOMAIN 2: Index Test (Xpert Ultra)

\section{DOMAIN 3: Reference Standard}

Is the reference standards likely to correctly classify the target condition? Yes

Were the reference standard results interpreted without knowledge of the Yes results of the index tests?

For rifampicin resistance testing, were the reference standard results inter- Yes preted without knowledge of the results of the index test?

Could the reference standard, its conduct, or its interpretation have in- Low risk
troduced bias?
Could the reference standard, its conduct, or its interpretation have in- troduced bias?

Are there concerns that the target condition as defined by the reference standard does not match the question?

\section{DOMAIN 4: Flow and Timing}

Was there an appropriate interval between index test and reference stan- Yes dard?

Did all patients receive the same reference standard? Yes

Were all patients included in the analysis? Yes

Could the patient flow have introduced bias?

Low risk

Peñata 2016

\section{Study characteristics}

Patient Sampling

Cross-sectional, consecutive, prospective and retrospective 
Peñata 2016 (Continued)

Patient characteristics and setting
Presenting signs and symptoms: patients with clinical suspicion of extrapulmonary tuberculosis

Age: mean 42 years (SD 19), range 1 to 91 years

Sex, female: $39 \%$

Children: $7 \%$

HIV infection: $40 \%$

Clinical setting: university hospital

Past history of TB: not reported

Patients on anti-TB treatment: no

Number of specimens evaluated: 236

Laboratory level: intermediate

Country: Colombia

World Bank Income Classification: middle income

High TB burden: no

High TB/HIV burden: no

High MDR-TB burden: no

\section{Xpert MTB/RIF}

WHO SOP or manufacturer's protocol followed: yes

Manufacturer's involvement: no

Target condition: lymph node TB, pleural TB, TB meningitis, peritoneal TB, pericardial TB, bone and joint TB

Reference standard TB detection: Ogawa medium

Reference standard rifampicin resistance detection: Ogawa-DST

Speciation: not reported

Decontamination: unclear

\section{Flow and timing}

Comparative

\section{Notes}

Methodological quality

\begin{tabular}{|c|c|c|c|}
\hline Item & $\begin{array}{l}\text { Authors' } \\
\text { judgement }\end{array}$ & Risk of bias & $\begin{array}{l}\text { Applicability } \\
\text { concerns }\end{array}$ \\
\hline
\end{tabular}

\section{DOMAIN 1: Patient Selection}


Peñata 2016 (Continued)

Was a consecutive or random sample of patients enrolled?

Was a case-control design avoided? Yes

Did the study avoid inappropriate exclusions? Yes

\section{Could the selection of patients have introduced bias?}

Are there concerns that the included patients and setting do not match the review question?

\section{DOMAIN 2: Index Test (Xpert MTB/RIF)}

Were the index test results interpreted without knowledge of the results of Yes the reference standard?

If a threshold was used, was it pre-specified? Yes

\section{Could the conduct or interpretation of the index test have introduced bias?

\section{DOMAIN 2: Index Test (Xpert Ultra)}

\section{DOMAIN 3: Reference Standard}

Is the reference standards likely to correctly classify the target condition? Unclear

Were the reference standard results interpreted without knowledge of the No results of the index tests?

For rifampicin resistance testing, were the reference standard results inter- No preted without knowledge of the results of the index test?

\section{Could the reference standard, its conduct, or its interpretation have in- High risk troduced bias?}

\section{Are there concerns that the target condition as defined by the reference standard does not match the question?

\section{DOMAIN 4: Flow and Timing}

Was there an appropriate interval between index test and reference stan- Yes dard?

Did all patients receive the same reference standard?

Yes

Were all patients included in the analysis?

Yes

\section{Could the patient flow have introduced bias?}

Low risk 
Perez-Risco 2018

\section{Study characteristics}

Patient Sampling

Patient characteristics and setting
Study design unclear, manner of patient selection not reported, retrospective

Presenting signs and symptoms: Smear-negative extrapulmonary patients

Age: adult

Sex, female: not reported

Children: 0\%

HIV infection: not reported

Clinical setting: laboratory-based evaluation

Past history of TB: not reported

Participants on anti-TB treatment: not reported

Number of specimens evaluated: CSF 3; pleural fluid 24; urine 24; bone or joint fluid 24

Laboratory level: central

Country: Spain

World Bank Income Classification: high

High TB burden: no

High TB/HIV burden: no

High MDR-TB burden: no

\section{Xpert Ultra}

WHO SOP or manufacturer's protocol followed: yes

Target condition: TB meningitis, pleural TB, genitourinary $\mathrm{TB}$, bone or joint TB

Reference standard TB detection: MGIT and LJ culture

Speciation: yes

Decontamination: no

Flow and timing

\section{Comparative}

\section{Methodological quality}

\begin{tabular}{lll}
\hline Item & $\begin{array}{l}\text { Authors' } \\
\text { judgement }\end{array}$ & Risk of bias $\begin{array}{l}\text { Applicability } \\
\text { concerns }\end{array}$ \\
\hline
\end{tabular}


Perez-Risco 2018 (Continued)

DOMAIN 1: Patient Selection

\begin{tabular}{ll}
\hline Was a consecutive or random sample of patients enrolled? & Unclear \\
\hline Was a case-control design avoided? & Unclear \\
\hline Did the study avoid inappropriate exclusions? & No \\
\hline Could the selection of patients have introduced bias? & High risk \\
\hline $\begin{array}{l}\text { Are there concerns that the included patients and setting do not match } \\
\text { the review question? }\end{array}$
\end{tabular}

DOMAIN 2: Index Test (Xpert MTB/RIF)

DOMAIN 2: Index Test (Xpert Ultra)

Were the index test results interpreted without knowledge of the results of Yes the reference standard?

If a threshold was used, was it pre-specified? Yes

\section{Could the conduct or interpretation of the index test have introduced bias?

Are there concerns that the index test, its conduct, or interpretation dif-

fer from the review question?

\section{DOMAIN 3: Reference Standard}

Is the reference standards likely to correctly classify the target condition? Yes

Were the reference standard results interpreted without knowledge of the Unclear results of the index tests?

For rifampicin resistance testing, were the reference standard results inter-

preted without knowledge of the results of the index test?

\section{Could the reference standard, its conduct, or its interpretation have in- troduced bias? standard does not match the question?

\section{DOMAIN 4: Flow and Timing}

Was there an appropriate interval between index test and reference stan- $\quad$ Yes dard?

Did all patients receive the same reference standard?

Were all patients included in the analysis?
Yes

Yes 
Rakotoarivelo 2018

\section{Study characteristics}

\section{Patient Sampling}

Patient characteristics and setting
Cohort, consecutive, prospective

Presenting signs and symptoms: patients with a febrile illness and chronic respiratory symptoms, pleural effusion, chronic abdominal pain or ascites, chronic meningitis, or other symptoms suggestive of extrapulmonary TB

Age: adult; mean (SD) 38.7 years (15.2)

Sex, female: $36 \%$

Children: no

HIV infection: $12 \%$

Clinical setting: tertiary care centre

Past history of TB: not reported

Participants on anti-TB treatment: not reported

Number of specimens evaluated: CSF: 77; pleural: 50

Laboratory level: central

Country: Madagascar

World Bank Income Classification: low income

High TB burden: no

High TB/HIV burden: no

High MDR-TB burden: no

\section{Xpert MTB/RIF}

WHO SOP or manufacturer's protocol followed: yes

Target condition and reference standard(s)

TB meningitis; pleural TB

Reference standard: LJ

Composite reference standard: proven and probable cases (culture-positive cases or clinical response to anti-TB treatment without any other diagnosis or other treatment)

Speciaiton: yes

decontamination: yes

\section{Flow and timing}

Comparative

\section{Notes}

The criteria of Marais were used for the diagnosis of tuberculous meningitis (Marais 2010). This classification and stratification of cases was independent of Xpert MTB/RIF and the panel was blinded to these results. 
Rakotoarivelo 2018 (Continued)

Methodological quality

\begin{tabular}{|c|c|c|c|}
\hline Item & $\begin{array}{l}\text { Authors' judge- } \\
\text { ment }\end{array}$ & Risk of bias & $\begin{array}{l}\text { Applicability con- } \\
\text { cerns }\end{array}$ \\
\hline
\end{tabular}

DOMAIN 1: Patient Selection

\begin{tabular}{lcl}
\hline Was a consecutive or random sample of patients enrolled? & Yes & Yes \\
\hline Was a case-control design avoided? & Yes & \\
\hline Did the study avoid inappropriate exclusions? & Low risk \\
\hline Could the selection of patients have introduced bias? & \\
\hline $\begin{array}{l}\text { Are there concerns that the included patients and setting do not } \\
\text { match the review question? }\end{array}$ & Unclear \\
\hline
\end{tabular}

\section{DOMAIN 2: Index Test (Xpert MTB/RIF)}

Were the index test results interpreted without knowledge of the re- Yes sults of the reference standard?

If a threshold was used, was it pre-specified? Yes

\section{Could the conduct or interpretation of the index test have introduced bias?

\section{DOMAIN 2: Index Test (Xpert Ultra)}

\section{DOMAIN 3: Reference Standard}

Is the reference standards likely to correctly classify the target con- Unclear dition?

Were the reference standard results interpreted without knowledge Yes of the results of the index tests?

For rifampicin resistance testing, were the reference standard re- Yes sults interpreted without knowledge of the results of the index test?

\section{Could the reference standard, its conduct, or its interpretation Unclear risk have introduced bias?}

\section{Are there concerns that the target condition as defined by the} reference standard does not match the question?

\section{DOMAIN 4: Flow and Timing}

Was there an appropriate interval between index test and reference Yes standard? 
Rakotoarivelo 2018 (Continued)

Were all patients included in the analysis?

Yes

\section{Rufai 2015}

\section{Study characteristics}

Patient Sampling

Cross-sectional, manner of participant selection not reported, prospective

Patient characteristics and setting

Presenting signs and symptoms: patients with high suspicion of pleural TB. Enrolment was based on standard clinical and radiological criteria, including a persistent cough of 2 weeks or longer, unexplained fever for 2 weeks or longer, unexplained weight loss with or without night sweats, chest pain, and radiological evidence of pleural effusion

Age: men: mean 42 years (SD 19 years); women: mean 39 years (SD 19 years)

Sex, female: $28 \%$

Children: $6 \%$

HIV infection: no

Clinical setting: tertiary care centre

Past history of TB: not reported

Patients on anti-TB treatment: no

Number of specimens evaluated: 161

Laboratory level: central

Country: India

World Bank Income Classification: middle income

High TB burden: yes

High TB/HIV burden: yes

High MDR-TB burden: yes

\section{Xpert MTB/RIF}

WHO SOP or manufacturer's protocol followed: no

Manufacturer's involvement: no

Target condition and reference standard(s)

Target condition: pleural TB

Reference standard TB detection: MGIT

Reference standard rifampicin resistance detection: MGITDST

Speciation: yes 
Rufai 2015 (Continued)

Flow and timing

Comparative

Notes

Methodological quality

\begin{tabular}{lll}
\hline Item & $\begin{array}{l}\text { Authors' judge- } \\
\text { ment }\end{array}$ & $\begin{array}{l}\text { Risk of bias } \\
\text { Applicability } \\
\text { concerns }\end{array}$ \\
\hline
\end{tabular}

DOMAIN 1: Patient Selection

\begin{tabular}{ll}
\hline Was a consecutive or random sample of patients enrolled? & Unclear \\
\hline Was a case-control design avoided? & Yes \\
\hline Did the study avoid inappropriate exclusions? & Yes
\end{tabular}

Could the selection of patients have introduced bias? Unclear risk

Are there concerns that the included patients and setting do not match the review question?

Unclear

\title{
DOMAIN 2: Index Test (Xpert MTB/RIF)
}

Were the index test results interpreted without knowledge of the re- Yes sults of the reference standard?

If a threshold was used, was it pre-specified? Yes

Could the conduct or interpretation of the index test have intro- Low risk
duced bias?
duced bias?

Are there concerns that the index test, its conduct, or interpretation differ from the review question?

\section{DOMAIN 2: Index Test (Xpert Ultra)}

\section{DOMAIN 3: Reference Standard}

Is the reference standards likely to correctly classify the target condi- Unclear tion?

Were the reference standard results interpreted without knowledge of Yes the results of the index tests?

For rifampicin resistance testing, were the reference standard results Yes interpreted without knowledge of the results of the index test?

\author{
Could the reference standard, its conduct, or its interpretation Unclear risk \\ have introduced bias?
} Are there concerns that the target condition as defined by the ref-
erence standard does not match the question? 
Rufai 2015 (Continued)

DOMAIN 4: Flow and Timing

Was there an appropriate interval between index test and reference Yes standard?

Did all patients receive the same reference standard? Yes

Were all patients included in the analysis?

Yes

Could the patient flow have introduced bias?

Low risk

\section{Rufai 2017a}

\section{Study characteristics}

Patient Sampling

Cross-sectional, manner of participant selection not reported, prospective

Patient characteristics and setting

Presenting signs and symptoms: patients with clinical or radiological suspicion of abdominal TB

Age: men: mean 41 years (SD 19 years); women: mean 46 years (SD 20 years)

Sex, female: $36 \%$

Children: no

HIV infection: no

Clinical setting: tertiary care centre

Past history of TB: not reported

Patients on anti-TB treatment: no

Number of specimens evaluated: 67

Laboratory level: central

Country: India

World Bank Income Classification: middle income

High TB burden: yes

High TB/HIV burden: yes

High MDR-TB burden: yes

Index tests

Xpert MTB/RIF

WHO SOP or manufacturer's protocol followed: no

Manufacturer's involvement: no

\footnotetext{
Target condition and reference standard(s)
}

\section{Target condition: peritoneal TB}

Reference standard TB detection: MGIT 
Rufai 2017a (Continued)

Reference standard rifampicin resistance detection: MGIT-DST

Decontamination: yes, NALC-NaOH

Speciation: yes

Flow and timing

Comparative

Notes

\section{Methodological quality}

\begin{tabular}{|c|c|c|c|}
\hline Item & $\begin{array}{l}\text { Authors' } \\
\text { judgement }\end{array}$ & Risk of bias & $\begin{array}{l}\text { Applicability } \\
\text { concerns }\end{array}$ \\
\hline
\end{tabular}

\section{DOMAIN 1: Patient Selection}

\begin{tabular}{lcl}
\hline Was a consecutive or random sample of patients enrolled? & Unclear \\
\hline Was a case-control design avoided? & Yes & Yes \\
\hline Did the study avoid inappropriate exclusions? & Unclear risk
\end{tabular}

Are there concerns that the included patients and setting do not match Unclear
the review question?

DOMAIN 2: Index Test (Xpert MTB/RIF)

Were the index test results interpreted without knowledge of the results of Yes the reference standard?

If a threshold was used, was it pre-specified? Yes

Could the conduct or interpretation of the index test have introduced Low risk
bias?

bias?

Are there concerns that the index test, its conduct, or interpretation differ from the review question?

\section{DOMAIN 2: Index Test (Xpert Ultra)}

\section{DOMAIN 3: Reference Standard}

Is the reference standards likely to correctly classify the target condition? Unclear

Were the reference standard results interpreted without knowledge of the Yes results of the index tests?

For rifampicin resistance testing, were the reference standard results inter- Yes preted without knowledge of the results of the index test?

\section{Could the reference standard, its conduct, or its interpretation have in- troduced bias? \\ Unclear risk}


Rufai 2017a (Continued)

\section{Are there concerns that the target condition as defined by the reference standard does not match the question?}

\section{DOMAIN 4: Flow and Timing}

Was there an appropriate interval between index test and reference stan- Yes dard?

Did all patients receive the same reference standard? Yes

Were all patients included in the analysis?

Yes

Could the patient flow have introduced bias?

Low risk

Rufai 2017b

\section{Study characteristics}

Patient Sampling

Cross-sectional, manner of participant selection not reported, prospective

Patient characteristics and setting

Presenting signs and symptoms: fatigue, malaise, low-grade fever, confusion, nausea and vomiting, lethargy, irritability, and unconsciousness

Age: men: mean 38 years (SD 10 years); women: mean 34 years (SD 22 years)

Sex, female: $41 \%$

Children: 6\%

HIV infection: not reported

Clinical setting: tertiary care centre

Past history of TB: not reported

Patients on anti-TB treatment: yes, $4 \%$

Number of specimens evaluated: 267

Laboratory level: central

Country: India

World Bank Income Classification: middle income

High TB burden: yes

High TB/HIV burden: yes

High MDR-TB burden: yes 
Rufai 2017b (Continued)

Target condition and reference standard(s)

\author{
Target condition: TB meningitis \\ Reference standard TB detection: MGIT \\ Reference standard rifampicin resistance detection: \\ MGIT-DST \\ Speciation: yes \\ Decontamination: no
}

Flow and timing

Comparative

Notes

Methodological quality

\begin{tabular}{lll}
\hline Item & $\begin{array}{l}\text { Authors' } \\
\text { judgement }\end{array}$ & $\begin{array}{c}\text { Risk of bias } \\
\text { Applicability } \\
\text { concerns }\end{array}$ \\
\hline
\end{tabular}

DOMAIN 1: Patient Selection

\begin{tabular}{lcl}
\hline Was a consecutive or random sample of patients enrolled? & Unclear \\
\hline Was a case-control design avoided? & Yes & Yes \\
\hline Did the study avoid inappropriate exclusions? & Unclear risk \\
\hline Could the selection of patients have introduced bias? & Low concern \\
\hline $\begin{array}{l}\text { Are there concerns that the included patients and setting do not match } \\
\text { the review question? }\end{array}$ &
\end{tabular}

\title{
DOMAIN 2: Index Test (Xpert MTB/RIF)
}

Were the index test results interpreted without knowledge of the results of Yes

the reference standard?

If a threshold was used, was it pre-specified? Yes
Could the conduct or interpretation of the index test have introduced
bias?
Low risk

Are there concerns that the index test, its conduct, or interpretation dif-

fer from the review question?

\section{DOMAIN 2: Index Test (Xpert Ultra)}

\section{DOMAIN 3: Reference Standard}

Is the reference standards likely to correctly classify the target condition? Yes

Were the reference standard results interpreted without knowledge of the Yes results of the index tests?

For rifampicin resistance testing, were the reference standard results inter- Yes preted without knowledge of the results of the index test? 
Rufai 2017b (Continued)

Could the reference standard, its conduct, or its interpretation have in-

Low risk troduced bias?

Are there concerns that the target condition as defined by the reference

Low concern standard does not match the question?

\section{DOMAIN 4: Flow and Timing}

Was there an appropriate interval between index test and reference stan- Yes dard?

Did all patients receive the same reference standard? Yes

Were all patients included in the analysis? Yes

Could the patient flow have introduced bias?

Low risk

Safianowska 2012

\section{Study characteristics}

Patient Sampling

Cross-sectional, consecutive, prospective

Patient characteristics and setting

Presenting signs and symptoms: not reported

Age: not reported

Sex, female: $46 \%$

Children: no

HIV infection: no

Clinical setting: university hospital

Past history of TB: not reported

Patients on anti-TB treatment: not reported

Number of specimens evaluated: 51

Laboratory level: intermediate

Country: Poland

World Bank Income Classification: high income

High TB burden: no

High TB/HIV burden: no

High MDR-TB burden: no

WHO SOP or manufacturer's protocol followed: yes

Manufacturer's involvement: no 
Safianowska 2012 (Continued)

Target condition and reference standard(s)

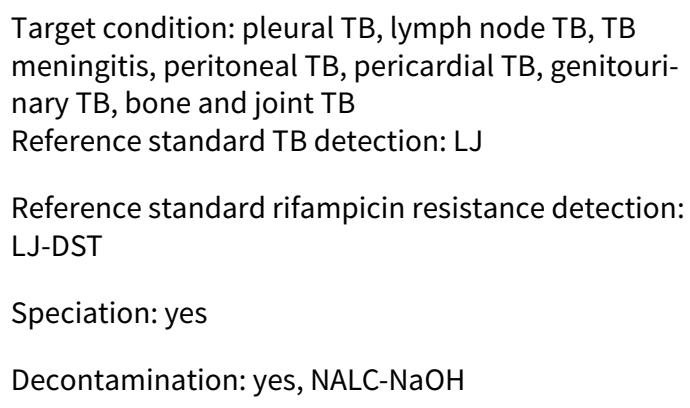

Target condition: pleural TB, lymph node TB, TB meningitis, peritoneal TB, pericardial TB, genitourinary $\mathrm{TB}$, bone and joint $\mathrm{TB}$ Reference standard TB detection: LJ

Reference standard rifampicin resistance detection: LJ-DST

Speciation: yes

Decontamination: yes, NALC-NaOH

\begin{tabular}{llll}
\hline Flow and timing & & \\
\hline Comparative & & \\
\hline Notes & Authors' & Risk of bias & Applicability \\
Methodological quality & judgement & concerns
\end{tabular}

DOMAIN 1: Patient Selection

\begin{tabular}{lcc}
\hline Was a consecutive or random sample of patients enrolled? & Yes & Yes \\
\hline Was a case-control design avoided? & Yes & Low risk \\
\hline Did the study avoid inappropriate exclusions? & Unclear \\
\hline Could the selection of patients have introduced bias? & \\
\hline $\begin{array}{l}\text { Are there concerns that the included patients and setting do not match } \\
\text { the review question? }\end{array}$
\end{tabular}

\section{DOMAIN 2: Index Test (Xpert MTB/RIF)}

Were the index test results interpreted without knowledge of the results of Yes the reference standard?

\section{Could the conduct or interpretation of the index test have introduced bias? fer from the review question?

\section{DOMAIN 2: Index Test (Xpert Ultra)}

\section{DOMAIN 3: Reference Standard}

Is the reference standards likely to correctly classify the target condition? Unclear

Were the reference standard results interpreted without knowledge of the No results of the index tests? 
Safianowska 2012 (Continued)

For rifampicin resistance testing, were the reference standard results inter- No preted without knowledge of the results of the index test?

\section{Could the reference standard, its conduct, or its interpretation have in-} troduced bias?

High risk

\section{Are there concerns that the target condition as defined by the reference} standard does not match the question?

Low concern

\section{DOMAIN 4: Flow and Timing}

Was there an appropriate interval between index test and reference stan- Yes dard?

Did all patients receive the same reference standard?

Yes

Were all patients included in the analysis?

Yes

Could the patient flow have introduced bias?

Low risk

Sarfaraz 2018

\section{Study characteristics}

Patient Sampling

Patient characteristics and setting
Cohort, manner of selection not reported, prospective

Presenting signs and symptoms: patients presenting with 1 or more superficial lymph nodes (i.e. cervical, axillary, and inguinal nodes) measuring $>2 \mathrm{~cm}$ in largest diameter and persisting for more than 1 month, with or without constitutional symptoms of fever, anorexia, and weight loss

Age: > 14 years of age; median 23 years (IQR 18 to 32 )

Sex, female: $79 \%$

Children: no

HIV infection: $1 \%$

Clinical setting: outpatient

Past history of TB: not reported

Participants on anti-TB treatment: not reported

Number of specimens evaluated: 261

Laboratory level: central

Country: Pakistan

World Bank Income Classification: middle income

High TB burden: yes

High TB/HIV burden: no 
Sarfaraz 2018 (Continued)

High MDR-TB burden: yes

Index tests

Xpert MTB/RIF

WHO SOP or manufacturer's protocol followed: yes

Target condition and reference standard(s)

Lymph node TB, tissue

MGIT; LJ

Composite reference standard includes histopathology

Rifampicin resistance

MGIT-DST

Speciation: not reported

Decontamination: yes (NALC-NaOH)

Flow and timing

Comparative

Notes

\section{Methodological quality}

\begin{tabular}{|c|c|c|c|}
\hline Item & $\begin{array}{l}\text { Authors' judge- } \\
\text { ment }\end{array}$ & Risk of bias & $\begin{array}{l}\text { Applicability } \\
\text { concerns }\end{array}$ \\
\hline
\end{tabular}

\section{DOMAIN 1: Patient Selection}

\begin{tabular}{ll}
\hline Was a consecutive or random sample of patients enrolled? & Unclear \\
\hline Was a case-control design avoided? & Yes \\
\hline Did the study avoid inappropriate exclusions? & Yes
\end{tabular}

\section{Could the selection of patients have introduced bias?}

Unclear risk

Are there concerns that the included patients and setting do not
match the review question?

\section{DOMAIN 2: Index Test (Xpert MTB/RIF)}

Were the index test results interpreted without knowledge of the results Yes of the reference standard?

If a threshold was used, was it pre-specified? Yes

Could the conduct or interpretation of the index test have intro- Low risk
duced bias?

Are there concerns that the index test, its conduct, or interpretation differ from the review question?

\section{DOMAIN 2: Index Test (Xpert Ultra)}


Sarfaraz 2018 (Continued)

\section{DOMAIN 3: Reference Standard}

Is the reference standards likely to correctly classify the target condi- Unclear tion?

Were the reference standard results interpreted without knowledge of Unclear the results of the index tests?

For rifampicin resistance testing, were the reference standard results Yes interpreted without knowledge of the results of the index test?

Could the reference standard, its conduct, or its interpretation have Unclear risk
introduced bias?

Are there concerns that the target condition as defined by the refer-
ence standard does not match the question?

\section{DOMAIN 4: Flow and Timing}

Was there an appropriate interval between index test and reference Yes standard?

\begin{tabular}{lcl}
\hline Did all patients receive the same reference standard? & Yes \\
\hline Were all patients included in the analysis? & Yes & Low risk \\
\hline Could the patient flow have introduced bias? & \\
\hline
\end{tabular}

\section{Study characteristics}

\begin{tabular}{ll}
\hline Patient Sampling & Cross-sectional, consecutive, prospective \\
\hline Patient characteristics and setting & Presenting signs and symptoms: not reported \\
Age: median 39 years, range $<1$ year to 96 years \\
Sex, female: $45 \%$
\end{tabular}


Scott 2014 (Continued)

$$
\begin{aligned}
& \text { High TB/HIV burden: yes } \\
& \text { High MDR-TB burden: yes }
\end{aligned}
$$

WHO SOP or manufacturer's protocol followed: yes for lymph node aspirate, pleural fluid, and peritoneal fluid; no for CSF

Manufacturer's involvement: no

\footnotetext{
Target condition and reference standard(s)
}
Target condition: pleural TB, lymph node TB, TB meningitis, peritoneal TB
Reference standard TB detection: MGIT
Reference standard rifampicin resistance detection: MGIT-DST and MTBDRplus
Speciation: yes
Decontamination: no

\begin{tabular}{llll}
\hline Flow and timing & \\
\hline Comparative & & \\
\hline Notes & Authors' & Risk of bias & Applicability \\
Item & judgement & concerns
\end{tabular}

\section{DOMAIN 1: Patient Selection}

\begin{tabular}{lcc}
\hline Was a consecutive or random sample of patients enrolled? & Yes & Yes \\
\hline Was a case-control design avoided? & Yes & Low risk \\
\hline Did the study avoid inappropriate exclusions?
\end{tabular}

Are there concerns that the included patients and setting do not match

Unclear the review question?

\section{DOMAIN 2: Index Test (Xpert MTB/RIF)}

Were the index test results interpreted without knowledge of the results of Yes the reference standard?

\begin{tabular}{|c|c|}
\hline $\begin{array}{l}\text { Could the conduct or interpretation of the index test have introduced } \\
\text { bias? }\end{array}$ & Low risk \\
\hline
\end{tabular}

If a threshold was used, was it pre-specified? Yes bias?

Are there concerns that the index test, its conduct, or interpretation differ from the review question? 
Scott 2014 (Continued)

DOMAIN 2: Index Test (Xpert Ultra)

\section{DOMAIN 3: Reference Standard}

Is the reference standards likely to correctly classify the target condition? Yes

Were the reference standard results interpreted without knowledge of the Yes results of the index tests?

For rifampicin resistance testing, were the reference standard results inter- Yes

preted without knowledge of the results of the index test?

\section{Could the reference standard, its conduct, or its interpretation have in- troduced bias? \\ Low risk}

Are there concerns that the target condition as defined by the reference

Low concern standard does not match the question?

DOMAIN 4: Flow and Timing

Was there an appropriate interval between index test and reference stan- Yes dard?

Did all patients receive the same reference standard? Yes

Were all patients included in the analysis?

Yes

Could the patient flow have introduced bias?

Low risk

Sharma 2014

\section{Study characteristics}

\begin{tabular}{ll}
\hline Patient Sampling & Cross-sectional, consecutive, prospective \\
\hline Patient characteristics and setting & $\begin{array}{l}\text { Presenting signs and symptoms: patients with clinical } \\
\text { suspicion of EPTB }\end{array}$ \\
Age: mean 35 years (SD 15 years) & Sex, female: $50 \%$ \\
Children: no & HIV infection: not reported \\
Clinical setting: tertiary care centre & Past history of TB: not reported \\
Patients on anti-TB treatment: no & Number of specimens evaluated: 1139 \\
Laboratory level: central & Country: India
\end{tabular}


High TB burden: yes

High TB/HIV burden: yes

High MDR-TB burden: yes

WHO SOP or manufacturer's protocol: yes for body fluids and LN tissue; no for CSF

Manufacturer's involvement: no

\section{Target condition and reference standard(s)}

Target condition: pleural TB, lymph node TB, TB meningitis, peritoneal $T B$, pericardial $T B$, genitourinary TB

Reference standard TB detection: LJ and MGIT

Reference standard rifampicin resistance detection: LJ-DST

Speciation: yes

Decontamination: yes, NALC-NaOH (for all specimens except CSF, pleural fluid, and urine)

\begin{tabular}{llll}
\hline Flow and timing & & \\
\hline Comparative & & \\
\hline Notes & Authors' & Risk of bias & $\begin{array}{l}\text { Applicability } \\
\text { concerns }\end{array}$
\end{tabular}

DOMAIN 1: Patient Selection

Was a consecutive or random sample of patients enrolled? Yes

Was a case-control design avoided? Yes

Did the study avoid inappropriate exclusions? Yes

Could the selection of patients have introduced bias? Low risk

Are there concerns that the included patients and setting do not match
the review question?

\section{DOMAIN 2: Index Test (Xpert MTB/RIF)}

Were the index test results interpreted without knowledge of the results of Yes the reference standard? 
Sharma 2014 (Continued)

Could the conduct or interpretation of the index test have introduced bias?

Are there concerns that the index test, its conduct, or interpretation differ from the review question?

\section{DOMAIN 2: Index Test (Xpert Ultra)}

\section{DOMAIN 3: Reference Standard}

Is the reference standards likely to correctly classify the target condition? Unclear

Were the reference standard results interpreted without knowledge of the Yes results of the index tests?

For rifampicin resistance testing, were the reference standard results inter- Yes preted without knowledge of the results of the index test?

\section{Could the reference standard, its conduct, or its interpretation have in- Unclear risk troduced bias?}

\section{Are there concerns that the target condition as defined by the reference} standard does not match the question?

\section{DOMAIN 4: Flow and Timing}

Was there an appropriate interval between index test and reference stan- $\quad$ Yes dard?

Did all patients receive the same reference standard? Yes

Were all patients included in the analysis? Yes

Could the patient flow have introduced bias? $\quad$ Low risk

Sharma 2016

\section{Study characteristics}

Patient Sampling

Cross-sectional, consecutive, prospective

Patient characteristics and setting

Presenting signs and symptoms: women being evaluated for infertility and suspected to have TB

Age: mean 29 years, range 19 to 41 years

Sex, female: $100 \%$

Children: no

HIV infection: not reported

Clinical setting: tertiary care centre

Past history of TB: not reported

Patients on anti-TB treatment: no 
Laboratory level: central

Country: India

World Bank Income Classification: middle income

High TB burden: yes

High TB/HIV burden: yes

High MDR-TB burden: yes

\title{
Xpert MTB/RIF
}

WHO SOP or manufacturer's protocol: yes

Manufacturer's involvement: no

\section{Target condition and reference standard(s)}

\author{
Target condition: genitourinary TB \\ MGIT-DST \\ Speciation: yes \\ Decontamination: yes, NALC-NaOH
}

Reference standard TB detection: LJ and MGIT

Reference standard rifampicin resistance detection:

Flow and timing

Comparative

Notes

\section{Methodological quality}

\begin{tabular}{lll}
\hline Item & $\begin{array}{l}\text { Authors' } \\
\text { judgement }\end{array}$ & $\begin{array}{c}\text { Risk of bias } \\
\text { Applicability } \\
\text { concerns }\end{array}$ \\
\hline
\end{tabular}

\section{DOMAIN 1: Patient Selection}

\begin{tabular}{lcc}
\hline Was a consecutive or random sample of patients enrolled? & Yes \\
\hline Was a case-control design avoided? & Yes & \\
\hline Did the study avoid inappropriate exclusions? & Low risk \\
\hline Could the selection of patients have introduced bias?
\end{tabular}

Are there concerns that the included patients and setting do not match Unclear
the review question?

DOMAIN 2: Index Test (Xpert MTB/RIF)

Were the index test results interpreted without knowledge of the results of Yes the reference standard? 
Sharma 2016 (Continued)

Could the conduct or interpretation of the index test have introduced

bias?

Are there concerns that the index test, its conduct, or interpretation dif-

Low concern fer from the review question?

\section{DOMAIN 2: Index Test (Xpert Ultra)}

\section{DOMAIN 3: Reference Standard}

Is the reference standards likely to correctly classify the target condition? Unclear

Were the reference standard results interpreted without knowledge of the Yes results of the index tests?

For rifampicin resistance testing, were the reference standard results inter- Yes preted without knowledge of the results of the index test?

\section{Could the reference standard, its conduct, or its interpretation have in- Unclear risk troduced bias?}

\section{Are there concerns that the target condition as defined by the reference} standard does not match the question?

\section{DOMAIN 4: Flow and Timing}

Was there an appropriate interval between index test and reference stan- Yes dard?

Did all patients receive the same reference standard? Yes

Were all patients included in the analysis? Yes

Could the patient flow have introduced bias?

Low risk

Sharma 2018

\section{Study characteristics}

Patient Sampling

Patient characteristics and setting
Cross-sectional, random selection, prospective

Presenting signs and symptoms: participants with persistent cough and unexplained fever for 2 weeks or more, unexplained weight loss, pleuritic chest pain, anorexia - among others, positive Mantoux test and the suggestive radiological findings

Age: mean 39 years (range, 18 to 60 )

Sex, female: $64 \%$

Children: no

HIV infection: $0 \%$

Clinical setting: university hospital 
Sharma 2018 (Continued)

\author{
Past history of TB: \\ Participants on anti-TB treatment: no \\ Number of specimens evaluated: 78 \\ Laboratory level: central \\ Country: India
}

World Bank Income Classification: middle-income

High TB burden: yes

High TB/HIV burden: yes

High MDR-TB burden: yes

\begin{tabular}{ll}
\hline Index tests & Xpert MTB/RIF \\
& WHO SOP or manufacturer's protocol: yes \\
\hline Target condition and reference standard(s) & $\begin{array}{l}\text { Pleural tuberculosis, pleural fluid } \\
\text { Composite reference standard: combination of smear, } \\
\text { culture, clinical findings, radiology, histology, cytol- } \\
\text { ogy, response to ATT }\end{array}$ \\
Speciation: yes \\
decontamination: no
\end{tabular}

Flow and timing

Comparative

Notes

\title{
Methodological quality
}

\begin{tabular}{lll}
\hline Item & $\begin{array}{l}\text { Authors' } \\
\text { judgement }\end{array}$ & $\begin{array}{c}\text { Risk of bias } \\
\text { Applicability } \\
\text { concerns }\end{array}$ \\
\hline
\end{tabular}

\section{DOMAIN 1: Patient Selection}

Was a consecutive or random sample of patients enrolled? Yes

\begin{tabular}{ll}
\hline Was a case-control design avoided? & Yes \\
\hline
\end{tabular}

Did the study avoid inappropriate exclusions? Yes

Could the selection of patients have introduced bias?

Low risk

Are there concerns that the included patients and setting do not match
the review question?

DOMAIN 2: Index Test (Xpert MTB/RIF)

Were the index test results interpreted without knowledge of the results of Yes the reference standard? 
Sharma 2018 (Continued)

If a threshold was used, was it pre-specified?

Yes

Could the conduct or interpretation of the index test have introduced Low risk
bias?

bias?

Are there concerns that the index test, its conduct, or interpretation dif-

fer from the review question?

Low concern

DOMAIN 2: Index Test (Xpert Ultra)

\section{DOMAIN 3: Reference Standard}

Is the reference standards likely to correctly classify the target condition? Yes

Were the reference standard results interpreted without knowledge of the Yes results of the index tests?

For rifampicin resistance testing, were the reference standard results interpreted without knowledge of the results of the index test?

\section{Could the reference standard, its conduct, or its interpretation have in-} troduced bias?

Are there concerns that the target condition as defined by the reference standard does not match the question?

\section{DOMAIN 4: Flow and Timing}

Was there an appropriate interval between index test and reference stan- Yes dard?

\begin{tabular}{lc}
\hline Did all patients receive the same reference standard? & Yes \\
\hline Were all patients included in the analysis? & Yes \\
\hline Could the patient flow have introduced bias? & Low risk \\
\hline
\end{tabular}

\section{Siddiqi 2019}

\section{Study characteristics}

\section{Patient Sampling}

Patient characteristics and setting
Cohort, manner of patient selection not reported, prospective

Presenting signs and symptoms: presented with signs and symptoms
concerning for TB meningitis and already received a lumbar puncture
as part of routine care
Age: age 18 years and older; TB meningitis culture positive: median 35
years (IQR 30 to 41 )
Sex, female: $36 \%$
Children: no
HIV infection: $86 \%$
Clinical setting: university teaching hospital 
Siddiqi 2019 (Continued)

Past history of TB: $20 \%$

Participants on anti-TB treatment: not reported

Number of specimens evaluated: 550

Laboratory level: central

Country: Zambia

World Bank Income Classification: middle income

High TB burden: yes

High TB/HIV burden: yes

High MDR-TB burden: no

\begin{tabular}{ll}
\hline Index tests & Xpert MTB/RIF \\
& WHO SOP or manufacturer's protocol followed: yes \\
\hline Target condition and reference standard(s) & TB meningitis \\
MGIT & Rifampicin resistance \\
& Samples found to be rifampin resistant by Xpert MTB/RIF had confirma- \\
& tory DST for rifampin and isoniazid conducted separately \\
& Speciation: yes \\
& Decontamination: No
\end{tabular}

Flow and timing

Comparative

Notes

After a lumbar puncture was completed, study staff identified patients from the microbiology laboratories who had $3 \mathrm{ml}$ of excess CSF remaining after routine testing composed of Gram stain, India ink stain, cryptococcal antigen testing, and bacterial culture on a blood agar plate.

A composite reference standard was defined as probable TB meningitis = patients with a CSF white blood cell count between 10 and 500, CSF total protein of $100 \mathrm{mg} / \mathrm{dl}$, and CSF glucose of $40 \mathrm{mg} / \mathrm{dl}$. These values were adapted from a uniform case definition of probable TBM for use in clinical research (Marais 2010). However, sensitivity and specificity were only determined using culture as the reference standard

\section{Methodological quality}

\begin{tabular}{llll}
\hline Item & Authors' judgement & Risk of bias & $\begin{array}{l}\text { Applicability con- } \\
\text { cerns }\end{array}$ \\
\hline
\end{tabular}

\section{DOMAIN 1: Patient Selection}

Was a consecutive or random sample of patients en- Unclear rolled? 
Siddiqi 2019 (Continued)

Did the study avoid inappropriate exclusions?

No

Could the selection of patients have introduced bias?

Are there concerns that the included patients and setting do not match the review question?

\section{DOMAIN 2: Index Test (Xpert MTB/RIF)}

Were the index test results interpreted without knowledge Yes of the results of the reference standard?

If a threshold was used, was it pre-specified?

Yes

\section{Could the conduct or interpretation of the index test} have introduced bias?

\section{Are there concerns that the index test, its conduct, or} interpretation differ from the review question?

\section{DOMAIN 2: Index Test (Xpert Ultra)}

\section{DOMAIN 3: Reference Standard}

\section{Is the reference standards likely to correctly classify the Yes} target condition?

\section{Were the reference standard results interpreted without Yes} knowledge of the results of the index tests?

For rifampicin resistance testing, were the reference stan- Unclear dard results interpreted without knowledge of the results of the index test?
Could the reference standard, its conduct, or its inter- pretation have introduced bias?

\section{Are there concerns that the target condition as defined by the reference standard does not match the ques-} tion?

\section{DOMAIN 4: Flow and Timing}

Was there an appropriate interval between index test and Yes reference standard?

\begin{tabular}{ll}
\hline Did all patients receive the same reference standard? & Yes \\
\hline Were all patients included in the analysis? & Yes \\
\hline
\end{tabular}

\section{Study characteristics}


Sun 2019 (Continued)

Patient Sampling

Patient characteristics and setting
Cohort, consecutive, prospective

Presenting signs and symptoms: patients with symptoms such as pain, swelling in the joints, tenderness, effusion, restriction of movements, and systematic symptoms such as fever, loss of weight/appetite, elevated erythrocyte sedimentation rate, and cough, breathlessness, and history of TB

Age: osteoarticular TB: median 51 years (range 16 to 86)

Sex, female: osteoarticular TB: 55\%

Children: no

HIV infection: $0 \%$

Clinical setting: national level TB referral centre

Past history of TB: not reported

Participants on anti-TB treatment: not reported

Number of specimens evaluated: 166

Laboratory level: central

Country: China

World Bank Income Classification: middle income

High TB burden: yes

High TB/HIV burden: yes

High MDR-TB burden: yes

Index tests

Xpert MTB/RIF and Xpert Ultra

WHO SOP or manufacturer's protocol followed: yes

\section{Target condition and reference standard(s)}

Bone or joint TB, fluid

\section{MGIT}

Composite reference standard: clinical, laboratory, histopathological, radiological and $\geq 6$ months' follow-up

Rifampicin resistance

LJ-DST

Speciation: yes

Decontamination: Yes

Flow and timing

\section{Comparative}


Sun 2019 (Continued)

Methodological quality

\begin{tabular}{lll}
\hline Item & $\begin{array}{l}\text { Authors' } \\
\text { judgement }\end{array}$ & Risk of bias $\begin{array}{l}\text { Applicability } \\
\text { concerns }\end{array}$ \\
\hline
\end{tabular}

DOMAIN 1: Patient Selection

Was a consecutive or random sample of patients enrolled?

Yes

Was a case-control design avoided? Yes

Did the study avoid inappropriate exclusions? Yes

Could the selection of patients have introduced bias? Low risk

Are there concerns that the included patients and setting do not match

Unclear the review question?

\section{DOMAIN 2: Index Test (Xpert MTB/RIF)}

Were the index test results interpreted without knowledge of the results of Yes the reference standard?

If a threshold was used, was it pre-specified? Yes

\section{Could the conduct or interpretation of the index test have introduced bias? \\ Low risk}

Are there concerns that the index test, its conduct, or interpretation differ from the review question?

\section{DOMAIN 2: Index Test (Xpert Ultra)}

Were the index test results interpreted without knowledge of the results of Yes the reference standard?

If a threshold was used, was it pre-specified?

Yes

\section{Could the conduct or interpretation of the index test have introduced bias?

Are there concerns that the index test, its conduct, or interpretation dif-

fer from the review question?

\section{DOMAIN 3: Reference Standard}

Is the reference standards likely to correctly classify the target condition? Unclear

Were the reference standard results interpreted without knowledge of the Yes results of the index tests?

For rifampicin resistance testing, were the reference standard results inter- Unclear preted without knowledge of the results of the index test?

\section{Could the reference standard, its conduct, or its interpretation have in- troduced bias?


Sun 2019 (Continued)

\section{Are there concerns that the target condition as defined by the reference standard does not match the question?}

\section{DOMAIN 4: Flow and Timing}

Was there an appropriate interval between index test and reference stan- Yes dard?

Did all patients receive the same reference standard? Yes

Were all patients included in the analysis?

Yes

\section{Study characteristics}

Patient Sampling

Patient characteristics and setting
Cross-sectional, consecutive, prospective

Presenting signs and symptoms: patients with signs and symptoms suggestive of extrapulmonary TB

Age: median 34 years

Sex, female: $39 \%$

Children: $0.06 \%$

HIV infection: $7 \%$

Clinical setting: tertiary care centre

Past history of TB: not reported

Patients on anti-TB treatment: not reported

Number of specimens evaluated: 215

Laboratory level: central

Country: India

World Bank Income Classification: middle income

High TB burden: yes

High TB/HIV burden: yes

High MDR-TB burden: yes

Index tests

WHO SOP or manufacturer's protocol followed: yes for lymph node tissue and pleural tissue; no for pleural fluid, bone and joint fluid, urine, peritoneal fluid, pericardial fluid, and CSF

Manufacturer's involvement: no 
Suzana 2016 (Continued)

Target condition and reference standard(s)
Target condition: pleural TB, lymph node TB, TB meningitis, peritoneal $T B$, pericardial TB, genitourinary TB, bone and joint TB

Reference standard TB detection: LJ and MGIT

Reference standard rifampicin resistance detection: LJDST and MGIT-DST

Speciation: yes

Decontamination: no

Flow and timing

\section{Comparative}

\section{Notes}

\section{Methodological quality}

\begin{tabular}{lll}
\hline Item & $\begin{array}{l}\text { Authors' judge- } \\
\text { ment }\end{array}$ & $\begin{array}{l}\text { Risk of bias } \\
\text { Applicability } \\
\text { concerns }\end{array}$ \\
\hline
\end{tabular}

DOMAIN 1: Patient Selection

\begin{tabular}{lc}
\hline Was a consecutive or random sample of patients enrolled? & Yes \\
\hline Was a case-control design avoided? & Yes \\
\hline Did the study avoid inappropriate exclusions? & Yes
\end{tabular}

Could the selection of patients have introduced bias? Low risk

Are there concerns that the included patients and setting do not
match the review question?
match the review question?

\section{DOMAIN 2: Index Test (Xpert MTB/RIF)}

Were the index test results interpreted without knowledge of the results Yes of the reference standard?
If a threshold was used, was it pre-specified?
Could the conduct or interpretation of the index test have intro- duced bias?
Low risk

Are there concerns that the index test, its conduct, or interpretation
differ from the review question?

DOMAIN 2: Index Test (Xpert Ultra)

\section{DOMAIN 3: Reference Standard}

Is the reference standards likely to correctly classify the target condi- Yes tion?

Were the reference standard results interpreted without knowledge of Yes the results of the index tests? 
Suzana 2016 (Continued)

For rifampicin resistance testing, were the reference standard results Yes interpreted without knowledge of the results of the index test?

\section{Could the reference standard, its conduct, or its interpretation have introduced bias?}

Low risk

\section{Are there concerns that the target condition as defined by the refer-} ence standard does not match the question?

Low concern

\section{DOMAIN 4: Flow and Timing}

Was there an appropriate interval between index test and reference Yes standard?

\begin{tabular}{lc}
\hline Did all patients receive the same reference standard? & Yes \\
\hline Were all patients included in the analysis? & Yes
\end{tabular}

Could the patient flow have introduced bias?

Low risk

Tadesse 2015

\section{Study characteristics}

Patient Sampling

Patient characteristics and setting
Cross-sectional, consecutive, prospective

Presenting signs and symptoms: people with presumptive lymph node TB

Age: $\leq 15$ years $15 \%$; > 15 years $85 \%$

Sex, female: $53 \%$

Children: $15 \%$

HIV infection: not reported

Clinical setting: university hospital (outpatient)

Past history of TB: not reported

Patients on anti-TB treatment: not reported

Number of specimens evaluated: 136

Laboratory level: central

Country: Ethiopia

World Bank Income Classification: low income

High TB burden: yes

High TB/HIV burden: yes

High MDR-TB burden: yes
Xpert MTB/RIF

WHO SOP or manufacturer's protocol followed: yes 
Reference standard rifampicin resistance detection: not reported

Speciation: yes

Decontamination: yes, NALC-NaOH

Flow and timing

Comparative

\begin{tabular}{|c|c|c|c|}
\hline Notes & \multicolumn{3}{|c|}{ Study used frozen specimens } \\
\hline \multicolumn{4}{|c|}{ Methodological quality } \\
\hline Item & $\begin{array}{l}\text { Authors' } \\
\text { judgement }\end{array}$ & Risk of bias & $\begin{array}{l}\text { Applicability } \\
\text { concerns }\end{array}$ \\
\hline
\end{tabular}

\section{DOMAIN 1: Patient Selection}

\begin{tabular}{lcc}
\hline Was a consecutive or random sample of patients enrolled? & Yes \\
\hline Was a case-control design avoided? & Yes \\
\hline Did the study avoid inappropriate exclusions? & Yes & Low risk \\
\hline Could the selection of patients have introduced bias? & \\
\hline
\end{tabular}

Are there concerns that the included patients and setting do not match the review question?

\section{DOMAIN 2: Index Test (Xpert MTB/RIF)}

Were the index test results interpreted without knowledge of the results of Yes the reference standard?

If a threshold was used, was it pre-specified? Yes

\begin{tabular}{|c|c|}
\hline $\begin{array}{l}\text { Could the conduct or interpretation of the index test have introduced } \\
\text { bias? }\end{array}$ & Low risk \\
\hline
\end{tabular}

Are there concerns that the index test, its conduct, or interpretation differ from the review question?

\section{DOMAIN 2: Index Test (Xpert Ultra)}

\section{DOMAIN 3: Reference Standard}

Is the reference standards likely to correctly classify the target condition? Unclear

Were the reference standard results interpreted without knowledge of the Yes results of the index tests? 
Tadesse 2015 (Continued)

For rifampicin resistance testing, were the reference standard results inter- Unclear preted without knowledge of the results of the index test?

\section{Could the reference standard, its conduct, or its interpretation have in- troduced bias? \\ Unclear risk}

\section{Are there concerns that the target condition as defined by the reference} standard does not match the question?

\section{DOMAIN 4: Flow and Timing}

Was there an appropriate interval between index test and reference stan- Yes dard?

Did all patients receive the same reference standard?

Yes

Were all patients included in the analysis?

Yes

Could the patient flow have introduced bias?

Low risk

Trajman 2014

\section{Study characteristics}

\begin{tabular}{|c|c|}
\hline Patient Sampling & Cross-sectional, consecutive, prospective \\
\hline \multirow[t]{15}{*}{ Patient characteristics and setting } & $\begin{array}{l}\text { Presenting signs and symptoms: patients with a pleural effusion } \\
\text { needing thoracentesis }\end{array}$ \\
\hline & Age: median 50 years (IQR 40 to 57) \\
\hline & Sex, female: $20 \%$ \\
\hline & Children: no \\
\hline & HIV infection: $5 \%$ \\
\hline & Clinical setting: secondary health facility (inpatient) \\
\hline & Past history of TB: not reported \\
\hline & Patients on anti-TB treatment: not reported \\
\hline & Number of specimens evaluated: 85 \\
\hline & Laboratory level: central \\
\hline & Country: Brazil \\
\hline & World Bank Income Classification: middle income \\
\hline & High TB burden: yes \\
\hline & High TB/HIV burden: yes \\
\hline & High MDR-TB burden: no \\
\hline \multirow[t]{2}{*}{ Index tests } & Xpert MTB/RIF \\
\hline & WHO SOP or manufacturer's protocol followed: yes \\
\hline
\end{tabular}


Trajman 2014 (Continued)

Manufacturer's involvement: no

Target condition and reference standard(s)

Target condition: pleural TB

Reference standard TB detection: MGIT

Reference standard rifampicin resistance detection: MGIT-DST

Speciation: not reported

Decontamination: no

Flow and timing

Comparative

Notes Patients were excluded if they had bleeding disorders contraindicating thoracentesis, if the fluid volume was insufficient for storage, or if a final diagnosis could not be ascertained. One of the main limitations of the study was the high number of presumptive (non-confirmed) cases. The number of exclusions was also high: out of 203 eligible patients, 110 were excluded: 21 did not have a final diagnosis and 89 did not have sufficient fluid to store. "Cultures of pleural tissue, which could significantly improve accuracy of diagnosis, were not performed"

Study used frozen specimens

\begin{tabular}{llll}
\hline Methodological quality & & \\
\hline Item & $\begin{array}{l}\text { Authors' judge- } \\
\text { ment }\end{array}$ & Risk of bias & $\begin{array}{l}\text { Applicability con- } \\
\text { cerns }\end{array}$ \\
\hline
\end{tabular}

DOMAIN 1: Patient Selection

\begin{tabular}{lcc}
\hline Was a consecutive or random sample of patients enrolled? & Yes \\
\hline Was a case-control design avoided? & Yes & \\
\hline Did the study avoid inappropriate exclusions? & Yes & Low risk \\
\hline Could the selection of patients have introduced bias? & Low concern \\
\hline $\begin{array}{l}\text { Are there concerns that the included patients and setting do } \\
\text { not match the review question? }\end{array}$ \\
\hline DOMAIN 2: Index Test (Xpert MTB/RIF)
\end{tabular}
Were the index test results interpreted without knowledge of Yes
the results of the reference standard?

If a threshold was used, was it pre-specified?

Yes

Could the conduct or interpretation of the index test have Low risk
introduced bias?

Are there concerns that the index test, its conduct, or interpretation differ from the review question? 
Trajman 2014 (Continued)

\section{DOMAIN 3: Reference Standard}

Is the reference standards likely to correctly classify the target Yes condition?

Were the reference standard results interpreted without knowl- Yes

edge of the results of the index tests?

For rifampicin resistance testing, were the reference standard results interpreted without knowledge of the results of the in-

dex test?

\section{Could the reference standard, its conduct, or its interpreta- tion have introduced bias?

Are there concerns that the target condition as defined by
the reference standard does not match the question?

\section{DOMAIN 4: Flow and Timing}

Was there an appropriate interval between index test and refer- Yes ence standard?

\begin{tabular}{lll}
\hline Did all patients receive the same reference standard? & Unclear \\
\hline Were all patients included in the analysis? & No & High risk \\
\hline Could the patient flow have introduced bias? & & H \\
\hline
\end{tabular}

\section{Ullah 2017}

\section{Study characteristics}

Patient Sampling Cross-sectional, consecutive, prospective

Patient characteristics and setting

Presenting signs and symptoms: patients meeting the following criteria: previously TB-treated cases with both positive and negative smears; failure of Cat-I and Cat-II TB drugs; all smear-positive cases that remained positive by the end of the second month of TB treatment; TB/HIV co-infection cases; seriously ill patients; contacts of MDR-TB patients

Age: mean 34 years (SD 19 years), range 3 to 80 years

Sex, female: $51 \%$

Children: $14 \%$

HIV infection: not reported

Clinical setting: tertiary care centre

Past history of TB: $60 \%$

Patients on anti-TB treatment: yes, percentage not reported

Number of specimens evaluated: 168 
Ullah 2017 (Continued)

Laboratory level: central

Country: Pakistan

World Bank Income Classification: middle income

High TB burden: yes

High TB/HIV burden: no

High MDR-TB burden: yes

\begin{tabular}{ll}
\hline Index tests & Xpert MTB/RIF \\
& WHO SOP or manufacturer's protocol followed: no \\
& Manufacturer's involvement: no \\
\hline Target condition and reference standard(s) & $\begin{array}{l}\text { Target condition: lymph node TB, TB meningitis, peritoneal TB, peri- } \\
\text { cardial TB } \\
\text { Reference standard TB detection: Middlebrook } 7 \mathrm{H} 10\end{array}$ \\
& $\begin{array}{l}\text { Reference standard rifampicin resistance detection: } \text { Middlebrook } \\
7 \text { H10 } \\
\text { Speciation: not reported } \\
\text { Decontamination: no }\end{array}$ \\
&
\end{tabular}

Flow and timing

Comparative

Notes

Study included a highly selective population that met specified criteria: previously TB-treated cases with both positive and negative smears; failure of Cat-I and Cat-II TB drugs; all smear-positive cases that remained positive by the end of the second month of TB treatment; TB/HIV co-infection cases; seriously ill patients; contacts of MDR-TB patients

\section{Methodological quality}

\begin{tabular}{llll}
\hline Item Authors' judgement & Risk of bias & $\begin{array}{l}\text { Applicability con- } \\
\text { cerns }\end{array}$
\end{tabular}

\section{DOMAIN 1: Patient Selection}

Was a consecutive or random sample of patients enrolled? Yes

\begin{tabular}{ll}
\hline Was a case-control design avoided? & Yes \\
\hline Did the study avoid inappropriate exclusions? & No \\
\hline
\end{tabular}

\section{Could the selection of patients have introduced bias?}

High risk

Are there concerns that the included patients and set-
ting do not match the review question?

\section{DOMAIN 2: Index Test (Xpert MTB/RIF)}


Ullah 2017 (Continued)

Were the index test results interpreted without knowledge Yes of the results of the reference standard?

If a threshold was used, was it pre-specified?

Yes

\section{Could the conduct or interpretation of the index test} have introduced bias?

\section{Are there concerns that the index test, its conduct, or in-}

terpretation differ from the review question?

DOMAIN 2: Index Test (Xpert Ultra)

\section{DOMAIN 3: Reference Standard}

Is the reference standards likely to correctly classify the tar- Yes get condition?

Were the reference standard results interpreted without Yes knowledge of the results of the index tests?

For rifampicin resistance testing, were the reference standard results interpreted without knowledge of the results of the index test?

Could the reference standard, its conduct, or its interpretation have introduced bias?

Are there concerns that the target condition as defined by the reference standard does not match the question?

\section{DOMAIN 4: Flow and Timing}

Was there an appropriate interval between index test and Yes reference standard?

\begin{tabular}{ll}
\hline Did all patients receive the same reference standard? & Yes \\
\hline Were all patients included in the analysis? & Yes \\
\hline Could the patient flow have introduced bias? & Low risk \\
\hline
\end{tabular}

Vadwai 2011

\section{Study characteristics}

Patient Sampling

Cross-sectional, consecutive, prospective

Patient characteristics and setting

Presenting signs and symptoms: suspected extrapulmonary TB based on symptoms: brain: irritability, restlessness, neck stiffness, headache persistent for 2 to 3 weeks, vomiting, seizures, changes in mental condition or behaviour; intestinal tract, abdomen: abdominal pain, diarrhoea; lymph nodes: enlargement of lymph nodes, mass formation in the neck; cardiorespiratory: shortness of breath, hypertension, chest pain, dyspnoea; 
Vadwai 2011 (Continued)

endometrium: pelvic pain, pelvic mass, irregular periods, infertility; skin

(cutaneous): visible presence of ulcers or lesions, tender nodules

Age: median 37 years

Sex, female: $15 \%$

Children: $3 \%$

HIV infection: $3 \%$

Clinical setting: tertiary care centre

Past history of TB: not reported

Patients on anti-TB treatment: no

Number of specimens evaluated: 60

Laboratory level: central

Country: India

World Bank Income Classification: middle income

High TB burden: yes

High TB/HIV burden: yes

High MDR-TB burden: yes

\section{Xpert MTB/RIF}

WHO SOP or manufacturer's protocol followed: yes for pleural fluid, peritoneal fluid, pericardial fluid; no for CSF

Manufacturer's involvement: yes, in design, analysis, or manuscript production (David Alland is among a group of co-investigators who invented molecular beacons and receive income from licensees, including to Cepheid, for $M$ tuberculosis detection)

\footnotetext{
Target condition and reference standard(s)
}

Target condition: pleural TB, TB meningitis, peritoneal TB, pericardial TB Reference standard TB detection: LJ and MGIT

Reference standard rifampicin resistance detection: MGIT-DST

Speciation: yes

Decontamination: yes, NALC-NaOH

\section{Flow and timing}

Comparative

Notes

"Patients were enrolled only if they could provide detailed clinical history and radiological and histology/cytology reports, along with an adequate amount of specimen material"

\section{Methodological quality}

\section{Item}

Authors' judgement

Risk of bias

\section{Applicability con-} cerns 
Vadwai 2011 (Continued)

\section{DOMAIN 1: Patient Selection}

Was a consecutive or random sample of patients en- Yes rolled?

\begin{tabular}{ll} 
Was a case-control design avoided? & Yes \\
\hline Did the study avoid inappropriate exclusions? & Yes \\
\hline
\end{tabular}

Could the selection of patients have introduced L L risk
bias?

bias?

Are there concerns that the included patients and setting do not match the review question?

\section{DOMAIN 2: Index Test (Xpert MTB/RIF)}

Were the index test results interpreted without knowl- Yes

edge of the results of the reference standard?

If a threshold was used, was it pre-specified? Yes

Could the conduct or interpretation of the index test Low risk
have introduced bias?

Are there concerns that the index test, its conduct, or interpretation differ from the review question?

\section{DOMAIN 2: Index Test (Xpert Ultra)}

\section{DOMAIN 3: Reference Standard}

\section{Is the reference standards likely to correctly classify the Unclear} target condition?

Were the reference standard results interpreted with- Yes out knowledge of the results of the index tests?

\section{For rifampicin resistance testing, were the reference Yes} standard results interpreted without knowledge of the results of the index test?
Could the reference standard, its conduct, or its in- terpretation have introduced bias?

\section{Are there concerns that the target condition as de- fined by the reference standard does not match the question?}

\section{DOMAIN 4: Flow and Timing}

Was there an appropriate interval between index test Yes and reference standard?

\begin{tabular}{ll}
\hline Did all patients receive the same reference standard? & Yes \\
\hline Were all patients included in the analysis? & Yes \\
\hline
\end{tabular}


Vadwai 2011 (Continued)

Van Rie 2013

\section{Study characteristics}

\section{Patient Sampling}

Patient characteristics and setting
Cross-sectional, consecutive, prospective

Presenting signs and symptoms: HIV-infected patients with suspicion of LNTB

Age: mean 36 years, range 18 to 73 years

Sex, female: $49 \%$

Children: no

HIV infection: $100 \%$

Clinical setting: tertiary care centre (inpatient and outpatient)

Past history of TB: not reported

Patients on anti-TB treatment: no

Number of specimens evaluated: 344

Laboratory level: central

Country: South Africa

World Bank Income Classification: middle income

High TB burden: yes

High TB/HIV burden: yes

High MDR-TB burden: yes
Xpert MTB/RIF

WHO SOP or manufacturer's protocol followed: yes

Manufacturer's involvement: no

Target condition: lymph node TB

Reference standard TB detection: MGIT

Reference standard rifampicin resistance detection: MGIT-DST

Speciation: yes

Decontamination: no

Flow and timing

Comparative 
Van Rie 2013 (Continued)

Notes

Methodological quality

\begin{tabular}{|c|c|c|c|}
\hline Item & $\begin{array}{l}\text { Authors' } \\
\text { judgement }\end{array}$ & Risk of bias & $\begin{array}{l}\text { Applicability } \\
\text { concerns }\end{array}$ \\
\hline
\end{tabular}

DOMAIN 1: Patient Selection

Was a consecutive or random sample of patients enrolled? Yes

Was a case-control design avoided? Yes

Did the study avoid inappropriate exclusions? Yes

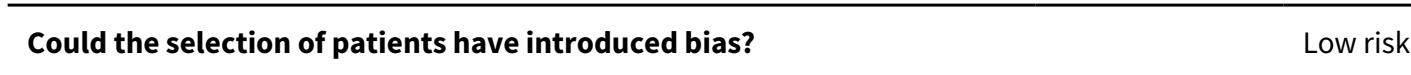

Are there concerns that the included patients and setting do not match Unclear

the review question?

DOMAIN 2: Index Test (Xpert MTB/RIF)

Were the index test results interpreted without knowledge of the results of Yes the reference standard?

If a threshold was used, was it pre-specified? Yes

Could the conduct or interpretation of the index test have introduced L L risk
bias?

bias?

Are there concerns that the index test, its conduct, or interpretation dif-

fer from the review question?

DOMAIN 2: Index Test (Xpert Ultra)

\section{DOMAIN 3: Reference Standard}

Is the reference standards likely to correctly classify the target condition? Yes

Were the reference standard results interpreted without knowledge of the Yes results of the index tests?

For rifampicin resistance testing, were the reference standard results inter- Yes preted without knowledge of the results of the index test?

Could the reference standard, its conduct, or its interpretation have in- Low risk
troduced bias?

Are there concerns that the target condition as defined by the reference Low concern
standard does not match the question?

\section{DOMAIN 4: Flow and Timing}

Was there an appropriate interval between index test and reference stan- Yes dard?

Did all patients receive the same reference standard?

Yes

Xpert MTB/RIF Ultra and Xpert MTB/RIF assays for extrapulmonary tuberculosis and rifampicin resistance in adults (Review) 
Van Rie 2013 (Continued)

Were all patients included in the analysis?

Yes

Could the patient flow have introduced bias?

Low risk

\section{Wang 2019}

\section{Study characteristics}

\section{Patient Sampling}

Patient characteristics and setting
Cohort, consecutive, prospective

Presenting signs and symptoms: adults who were offered lumbar puncture as a part of routine care for suspected brain infection; TB symptoms, chest pain, radiological evidence of pleural effusion, thoracoscopic examination that suggested TB

Age: 15 years and older; pleural tuberculosis TB 37 years (range, 15 to 89); TB meningitis 33 years (range, 15 to 83$)$

Sex, female: pleural tuberculosis: $20 \%$; TB meningitis: $44 \%$

Children: no

HIV infection: $0 \%$

Clinical setting: national level tuberculosis referral centre

Past history of TB: not reported

Participants on anti-TB treatment: not reported

Number of specimens evaluated: not reported

Laboratory level: central

Country: China

World Bank Income Classification: middle income

High TB burden country: yes

High MDR-TB burden country: yes

High TB/HIV burden country: yes

Prevalence of TB cases in the study:

Index tests Xpert MTB/RIF and Xpert Ultra

Target condition and reference standard(s)

\section{LJ and MGIT \\ Composite reference standard for pleural TB: com- posed of clinical, laboratory, histopathological, and radiological and follow-up features \\ Rifampicin resistance \\ LJ-DST}


Wang 2019 (Continued)

\section{Speciation: not reported}

Decontamination: no

Flow and timing

Comparative

Notes

Methodological quality

\begin{tabular}{lll}
\hline Item & $\begin{array}{l}\text { Authors' } \\
\text { judgement }\end{array}$ & $\begin{array}{c}\text { Risk of bias } \\
\text { Applicability } \\
\text { concerns }\end{array}$ \\
\hline
\end{tabular}

DOMAIN 1: Patient Selection

Was a consecutive or random sample of patients enrolled?

Was a case-control design avoided? Yes

Did the study avoid inappropriate exclusions?

Yes

Could the selection of patients have introduced bias?

Low risk

Are there concerns that the included patients and setting do not match the review question?

Unclear

\section{DOMAIN 2: Index Test (Xpert MTB/RIF)}

Were the index test results interpreted without knowledge of the results of Yes the reference standard?

If a threshold was used, was it pre-specified?

Yes

Could the conduct or interpretation of the index test have introduced L risk
bias?
bias?

Are there concerns that the index test, its conduct, or interpretation differ from the review question?

\section{DOMAIN 2: Index Test (Xpert Ultra)}

Were the index test results interpreted without knowledge of the results of Yes the reference standard?

If a threshold was used, was it pre-specified? Yes

Could the conduct or interpretation of the index test have introduced Low risk
bias?

Are there concerns that the index test, its conduct, or interpretation differ from the review question?

DOMAIN 3: Reference Standard

Is the reference standards likely to correctly classify the target condition? Yes 
Wang 2019 (Continued)

Were the reference standard results interpreted without knowledge of the Unclear results of the index tests?

For rifampicin resistance testing, were the reference standard results inter- Unclear preted without knowledge of the results of the index test?

Could the reference standard, its conduct, or its interpretation have in- Unclear risk
troduced bias?
troduced bias?

\section{Are there concerns that the target condition as defined by the reference} standard does not match the question?

\section{DOMAIN 4: Flow and Timing}

Was there an appropriate interval between index test and reference stan- Yes dard?

\begin{tabular}{ll}
\hline Did all patients receive the same reference standard? Yes \\
\hline
\end{tabular}

Were all patients included in the analysis? Yes

\section{Could the patient flow have introduced bias?}

Low risk

Wang 2020

\section{Study characteristics}

\begin{tabular}{|c|c|}
\hline Patient Sampling & Cohort, consecutive, prospective \\
\hline \multirow[t]{15}{*}{ Patient characteristics and setting } & $\begin{array}{l}\text { Presenting signs and symptoms: suspected pleural tu- } \\
\text { berculosis }\end{array}$ \\
\hline & Age: median 45 years; range: 15 to 89 \\
\hline & Sex, female: $32.5 \%$ \\
\hline & Children: no \\
\hline & HIV infection: $0 \%$ \\
\hline & Clinical setting: Unclear \\
\hline & Past history of TB: not reported \\
\hline & Participants on anti-TB treatment: not reported \\
\hline & Number of specimens evaluated: 139 \\
\hline & Laboratory level: central \\
\hline & Country: China \\
\hline & World Bank Income Classification: middle income \\
\hline & High TB burden country: yes \\
\hline & High MDR-TB burden country: yes \\
\hline & High TB/HIV burden country: yes \\
\hline
\end{tabular}


Wang 2020 (Continued)

Index tests

Xpert MTB/RIF and Xpert Ultra

WHO SOP or manufacturer's protocol followed: yes

Manufacturer's involvement: no

Target condition and reference standard(s)

\section{Pleural TB}

LJ and MGIT

Speciation: Yes

Decontamination: no

Flow and timing

Comparative

Notes

This study used fresh specimens for Xpert and frozen specimens for Xpert Ultra

\section{Methodological quality}

\begin{tabular}{|c|c|c|c|}
\hline Item & $\begin{array}{l}\text { Authors' } \\
\text { judgement }\end{array}$ & Risk of bias & $\begin{array}{l}\text { Applicability } \\
\text { concerns }\end{array}$ \\
\hline
\end{tabular}

\section{DOMAIN 1: Patient Selection}

\begin{tabular}{lcl}
\hline Was a consecutive or random sample of patients enrolled? & Yes \\
\hline Was a case-control design avoided? & Yes \\
\hline Did the study avoid inappropriate exclusions? & No & Low risk
\end{tabular}

Are there concerns that the included patients and setting do not match
the review question?

DOMAIN 2: Index Test (Xpert MTB/RIF)

Were the index test results interpreted without knowledge of the results of the reference standard?

If a threshold was used, was it pre-specified?

\section{Could the conduct or interpretation of the index test have introduced} bias?

\section{Are there concerns that the index test, its conduct, or interpretation dif-}

fer from the review question?

\section{DOMAIN 2: Index Test (Xpert Ultra)}

Were the index test results interpreted without knowledge of the results of Yes the reference standard? 
Wang 2020 (Continued)

Could the conduct or interpretation of the index test have introduced

Low risk bias?

Are there concerns that the index test, its conduct, or interpretation dif-

Low concern fer from the review question?

\section{DOMAIN 3: Reference Standard}

Is the reference standards likely to correctly classify the target condition? Yes

Were the reference standard results interpreted without knowledge of the Unclear results of the index tests?

For rifampicin resistance testing, were the reference standard results inter- Yes preted without knowledge of the results of the index test?

\section{Could the reference standard, its conduct, or its interpretation have in- Unclear risk troduced bias?}

\section{Are there concerns that the target condition as defined by the reference} standard does not match the question?

\section{DOMAIN 4: Flow and Timing}

Was there an appropriate interval between index test and reference stan- Yes dard?

\begin{tabular}{ll}
\hline Did all patients receive the same reference standard? Yes
\end{tabular}

Were all patients included in the analysis?

Yes

\section{Could the patient flow have introduced bias?}

Low risk

\section{Wu 2019}

\section{Study characteristics}

Patient Sampling

Cohort, manner of selection not reported, consecutive

Patient characteristics and setting

Presenting signs and symptoms: patients with suspi-
cion of extrapulmonary tuberculosis; diagnostic cri-
teria followed the WHO guidelines and was based on
a combination of clinical symptoms, radiological evi-
dence compatible with active TB, histological obser-
vations, lack of improvement in response to a course
of broad-spectrum antibiotics
Age: 16 years and older
Sex, female: $32 \%$
Children: no
HIV infection: $0 \%$
Clinical setting: tertiary-care hospital

Xpert MTB/RIF Ultra and Xpert MTB/RIF assays for extrapulmonary tuberculosis and rifampicin resistance in adults (Review)

Collaboration. 
Wu 2019 (Continued)

Past history of TB: not reported

Participants on anti-TB treatment: not reported

Number of specimens evaluated: lymph node fluid 52; pleural fluid: 119

Laboratory level: central

Country: China

World Bank Income Classification: middle income

High TB burden: yes

High TB/HIV burden: yes

High MDR-TB burden: yes

\begin{tabular}{ll} 
Index tests & $\begin{array}{l}\text { Xpert MTB/RIF and Xpert Ultra } \\
\text { WHO SOP or manufacturer's protocol followed: no } \\
\text { (see note) }\end{array}$ \\
\hline Target condition and reference standard(s) & $\begin{array}{l}\text { Lymph node tuberculosis; pleural tuberculosis } \\
\text { MGIT }\end{array}$ \\
$\begin{array}{l}\text { Speciation: not reported } \\
\text { Sterile specimens were directly processed. Non-ster- } \\
\text { ile specimens were pretreated with N-acetyl-L-cys- } \\
\text { teine-NaOH-Na citrate }\end{array}$
\end{tabular}

Flow and timing

Comparative

Notes

$4 \mathrm{ml}$ GeneXpert sample reagent was added to the remaining $1 \mathrm{ml}$ of each specimen

\section{Methodological quality}

\begin{tabular}{|c|c|c|c|}
\hline Item & $\begin{array}{l}\text { Authors' } \\
\text { judgement }\end{array}$ & Risk of bias & $\begin{array}{l}\text { Applicability } \\
\text { concerns }\end{array}$ \\
\hline
\end{tabular}

\section{DOMAIN 1: Patient Selection}

\begin{tabular}{lcc}
\hline Was a consecutive or random sample of patients enrolled? & Unclear \\
\hline Was a case-control design avoided? & Yes & Yes \\
\hline Did the study avoid inappropriate exclusions? & Unclear risk \\
\hline Could the selection of patients have introduced bias? & Unclear \\
\hline $\begin{array}{l}\text { Are there concerns that the included patients and setting do not match } \\
\text { the review question? }\end{array}$ & \\
\hline
\end{tabular}

\section{DOMAIN 2: Index Test (Xpert MTB/RIF)}


Wu 2019 (Continued)

Were the index test results interpreted without knowledge of the results of Yes the reference standard?

If a threshold was used, was it pre-specified?

Yes

Could the conduct or interpretation of the index test have introduced bias?

Are there concerns that the index test, its conduct, or interpretation dif-

fer from the review question?

DOMAIN 2: Index Test (Xpert Ultra)

Were the index test results interpreted without knowledge of the results of Yes the reference standard?

If a threshold was used, was it pre-specified?

Could the conduct or interpretation of the index test have introduced

bias?

Are there concerns that the index test, its conduct, or interpretation dif-

fer from the review question?

\section{DOMAIN 3: Reference Standard}

Is the reference standards likely to correctly classify the target condition? Yes

Were the reference standard results interpreted without knowledge of the Yes results of the index tests?

For rifampicin resistance testing, were the reference standard results interpreted without knowledge of the results of the index test?

\section{Could the reference standard, its conduct, or its interpretation have in- troduced bias?

\section{DOMAIN 4: Flow and Timing}

Was there an appropriate interval between index test and reference stan- Yes dard?

Did all patients receive the same reference standard?

Were all patients included in the analysis?
Yes

Yes

\section{Study characteristics}


Zeka 2011 (Continued)

Patient Sampling

Cross-sectional, consecutive, retrospective

Patient characteristics and setting

Presenting signs and symptoms: clinical findings of possible TB

Age: median 48 years

Sex, female: $42 \%$

Children: $13 \%$

HIV infection: $1 \%$

Clinical setting: tertiary care centre

Past history of TB: not reported

Patients on anti-TB treatment: no

Number of specimens evaluated: 149

Laboratory level: central

Country: Turkey

World Bank Income Classification: middle income

High TB burden: no

High TB/HIV burden: no

High MDR-TB burden: no

WHO SOP or manufacturer's protocol followed: no

Manufacturer's involvement: no

Target condition and reference standard(s)

Target condition: pleural TB, TB meningitis, genitouri-
nary TB, peritoneal TB, pericardial TB
Reference standard TB detection: LJ and BacT liquid
medium
Reference standard rifampicin resistance detection:
$7 \mathrm{H} 10$ agar media
Speciation: yes
Decontamination: no

Flow and timing

\section{Comparative}

\begin{tabular}{|c|c|c|c|}
\hline Notes & \multicolumn{3}{|c|}{ Study used frozen specimens } \\
\hline \multicolumn{4}{|c|}{ Methodological quality } \\
\hline Item & $\begin{array}{l}\text { Authors' } \\
\text { judgement }\end{array}$ & Risk of bias & $\begin{array}{l}\text { Applicability } \\
\text { concerns }\end{array}$ \\
\hline
\end{tabular}


Zeka 2011 (Continued)

DOMAIN 1: Patient Selection

\begin{tabular}{ll}
\hline Was a consecutive or random sample of patients enrolled? & Yes \\
\hline Was a case-control design avoided? & Yes \\
\hline Did the study avoid inappropriate exclusions? & Yes risk \\
\hline Could the selection of patients have introduced bias? & Unclear \\
\hline $\begin{array}{l}\text { Are there concerns that the included patients and setting do not match } \\
\text { the review question? }\end{array}$
\end{tabular}

\section{DOMAIN 2: Index Test (Xpert MTB/RIF)}

Were the index test results interpreted without knowledge of the results of Yes the reference standard?

If a threshold was used, was it pre-specified?

Yes

\section{Could the conduct or interpretation of the index test have introduced L L risk bias?}

Are there concerns that the index test, its conduct, or interpretation differ from the review question?

DOMAIN 2: Index Test (Xpert Ultra)

\section{DOMAIN 3: Reference Standard}

Is the reference standards likely to correctly classify the target condition? Yes

Were the reference standard results interpreted without knowledge of the Yes results of the index tests?

For rifampicin resistance testing, were the reference standard results inter- No preted without knowledge of the results of the index test?

\section{Could the reference standard, its conduct, or its interpretation have in- Low risk troduced bias?}

Are there concerns that the target condition as defined by the reference standard does not match the question?

\section{DOMAIN 4: Flow and Timing}

Was there an appropriate interval between index test and reference stan- Yes dard?

Did all patients receive the same reference standard?

Were all patients included in the analysis?
Yes

Yes 
Zmak 2013

\section{Study characteristics}

\section{Patient Sampling}

Cross-sectional, manner of participant selection not reported, prospective

\section{Patient characteristics and setting}

Presenting signs and symptoms: patients suspected of EPTB

Age: 15 years and older

Sex, female: not reported

Children: $13 \%$

HIV infection: not reported

Clinical setting: laboratory-based evaluation

Past history of TB: not reported

Patients on anti-TB treatment: not reported

Number of specimens evaluated: 176

Laboratory level: central

Country: Croatia

World Bank Income Classification: high income

High TB burden: no

High TB/HIV burden: no

High MDR-TB burden: no

WHO SOP or manufacturer's protocol followed: yes for pleural fluid, urine, peritoneal fluid, pericardial fluid, and blood; no for CSF

Manufacturer's involvement: no pericardial TB, genitourinary $T B$, disseminated TB Reference standard TB detection: LJ, Stonebrink, and MGIT

Reference standard rifampicin resistance detection: LJ-DST

Speciation: yes

Decontamination: no

\section{Flow and timing}

\section{Notes}

"Although the NRL performs a third-level laboratory service for the whole country, it is actually also involved in first and second-level laboratory work for several counties" 
Zmak 2013 (Continued)

\begin{tabular}{|c|c|c|c|}
\hline It & Authors' judge- & Risk of bias & $\begin{array}{l}\text { Applicability con- } \\
\text { cerns }\end{array}$ \\
\hline
\end{tabular}

DOMAIN 1: Patient Selection

\begin{tabular}{lcc}
\hline Was a consecutive or random sample of patients enrolled? & Unclear \\
\hline Was a case-control design avoided? & Yes & Yes \\
\hline Did the study avoid inappropriate exclusions? & & Unclear risk \\
\hline Could the selection of patients have introduced bias? & \\
\hline
\end{tabular}

Are there concerns that the included patients and setting do not
match the review question?

\section{DOMAIN 2: Index Test (Xpert MTB/RIF)}

Were the index test results interpreted without knowledge of the re- Yes sults of the reference standard?

\begin{tabular}{l}
\hline If a threshold was used, was it pre-specified? \\
$\begin{array}{l}\text { Could the conduct or interpretation of the index test have intro- } \\
\text { duced bias? }\end{array}$ \\
$\begin{array}{l}\text { Are there concerns that the index test, its conduct, or interpre- } \\
\text { tation differ from the review question? }\end{array}$ \\
\hline DOMAIN 2: Index Test (Xpert Ultra)
\end{tabular}

\section{DOMAIN 3: Reference Standard}

Is the reference standards likely to correctly classify the target con- Yes dition?

Were the reference standard results interpreted without knowledge No of the results of the index tests?

For rifampicin resistance testing, were the reference standard re- No sults interpreted without knowledge of the results of the index test?

Could the reference standard, its conduct, or its interpretation
have introduced bias?
have introduced bias?

Are there concerns that the target condition as defined by the
reference standard does not match the question?

\section{DOMAIN 4: Flow and Timing}

Was there an appropriate interval between index test and reference Yes standard?

\begin{tabular}{ll}
\hline Did all patients receive the same reference standard? & Yes \\
\hline Were all patients included in the analysis? & Yes \\
\hline
\end{tabular}


Zmak 2013 (Continued)

CSF: cerebrospinal fluid; DST: drug susceptibility testing; EBUS: endobronchial ultrasound; EPTB: extrapulmonary tuberculosis: IQR: interquartile ratio; LJ: Löwenstein-Jensen; LN: lymph node; MDR-TB: multi-drug-resistant tuberculosis; MGIT: mycobacteria growth indicator tube; NALC-NaOH: N-acetyl-L-cysteine-sodium hydroxide; SD: standard deviation; SOP: standard operating procedure; TB: tuberculosis; TBM: tuberculous meningitis; WHO: World Health Organization.

Characteristics of excluded studies [ordered by study ID]

\begin{tabular}{|c|c|}
\hline Study & Reason for exclusion \\
\hline Abong 2019 & Test other than Xpert MTB/RIF and Xpert Ultra \\
\hline Adejumo 2018 & Did not contain specimen for extrapulmonary TB \\
\hline Afsar 2018 & Did not contain data by site for extrapulmonary TB \\
\hline Ahmad 2018 & Inadequate reference standard \\
\hline Akhter 2019 & Test other than Xpert MTB/RIF and Xpert Ultra \\
\hline Ali 2018 & Inadequate reference standard \\
\hline Allahyartorkaman 2019 & Test other than Xpert MTB/RIF and Xpert Ultra \\
\hline Alvarez Uria 2012 & Inappropriate reference standard \\
\hline Andrey 2015 & Case report \\
\hline Armand 2011 & Case-control study \\
\hline Arockiaraj 2015 & Abstract; we included the published study (Arockiaraj 2017) in the review \\
\hline Arockiaraj 2017 & Includes both adults and children or no information about age of enrolment \\
\hline Arockiaraj 2019a & Case-control study \\
\hline Arockiaraj 2019b & Children \\
\hline Atherton 2018 & Case report \\
\hline Aydemir 2019 & Could not obtain; same publication as Terzi 2019 \\
\hline Bablishvili 2015 & Did not contain specimen for extrapulmonary TB \\
\hline Bahr 2018a & Test other than Xpert MTB/RIF and Xpert Ultra \\
\hline Bahr 2018b & Duplicate data for Bahr 2017 \\
\hline Bahr 2019 & Review \\
\hline Baikunje 2019 & Test other than Xpert MTB/RIF and Xpert Ultra \\
\hline Bajrami 2016 & Could not extract $2 \times 2$ values \\
\hline
\end{tabular}




\begin{tabular}{|c|c|}
\hline Study & Reason for exclusion \\
\hline Balcha 2014 & Did not contain specimen for extrapulmonary TB \\
\hline Bankar 2018 & Includes both adults and children or no information about age of enrolment \\
\hline Bemba 2017 & Inappropriate reference standard \\
\hline Ben Saad 2018 & Could not extract $2 \times 2$ values \\
\hline Bhardwaj 2019 & Test other than Xpert MTB/RIF and Xpert Ultra \\
\hline Bhatia 2016 & Could not extract $2 \times 2$ values \\
\hline Bholla 2016 & Children \\
\hline Biadglegne 2013 & Could not extract $2 \times 2$ values \\
\hline Bilgin 2016 & Could not extract $2 \times 2$ values \\
\hline Borraz-Noriega 2018 & Could not extract $2 \times 2$ values \\
\hline Boyles 2018 & Correspondence without original data \\
\hline Bunsow 2014 & Could not extract $2 \times 2$ values \\
\hline Celik 2015 & Could not extract $2 \times 2$ values \\
\hline Chaidir 2018 & Inappropriate reference standard \\
\hline Chakraborty 2019 & Xpert Ultra was not evaluated \\
\hline Chen 2016 & Could not extract $2 \times 2$ values \\
\hline Chhajed 2019 & Xpert Ultra was not evaluated \\
\hline Christopher 2018 & Could not extract $2 \times 2$ values \\
\hline Coetzee 2014 & Children \\
\hline Coleman 2015 & Case-control study \\
\hline Creswell 2019 & Did not contain specimen for extrapulmonary TB \\
\hline Dahale 2019 & Case-control study \\
\hline Das 2019 & Children \\
\hline Deggim 2013 & Fewer than 5 specimens for a given type of specimen (only 1 pleural fluid specimen) \\
\hline Dharan 2016 & Did not contain specimen for extrapulmonary TB \\
\hline Diallo 2016 & Includes both adults and children or no information about age of enrolment \\
\hline Diop 2016 & Inappropriate reference standard \\
\hline Edwards 2016 & Case report \\
\hline
\end{tabular}




\begin{tabular}{|c|c|}
\hline Study & Reason for exclusion \\
\hline Ejeta 2018 & Could not extract $2 \times 2$ values \\
\hline Erdem 2014 & Index test other than Xpert MTB/RIF \\
\hline Fanosie 2016 & Did not contain specimen for extrapulmonary TB \\
\hline Fantahun 2019 & Test other than Xpert MTB/RIF and Xpert Ultra \\
\hline Floridia 2017 & Could not extract $2 \times 2$ values \\
\hline García 2017 & Duplicate data for García Cañete 2017 \\
\hline García Cañete 2017 & Includes both adults and children or no information about age of enrolment \\
\hline Gascoyne-Binzi 2012 & Abstract; we could not extract data by form of extrapulmonary TB \\
\hline Gati 2018 & Could not extract $2 \times 2$ values \\
\hline Gautam 2018 & Inappropriate reference standard \\
\hline Gautam 2019 & Inappropriate reference standard \\
\hline Gounden 2018 & Could not extract $2 \times 2$ values \\
\hline Gulla 2019 & Could not extract $2 \times 2$ values \\
\hline Gursoy 2016 & Includes both adults and children or no information about age of enrolment \\
\hline Habeenzu 2017 & Did not contain specimen for extrapulmonary TB \\
\hline Habous 2019 & Includes both adults and children or no information about age of enrolment \\
\hline Hanifa 2017 & Could not extract $2 \times 2$ values \\
\hline Held 2014 & Bone tissue, specimen not included \\
\hline Held 2016 & Bone tissue, specimen not included \\
\hline Held 2017 & Bone tissue, specimen not included \\
\hline Ioannidis 2010 & Duplicate data \\
\hline Ioannidis 2011 & Includes both adults and children or no information about age of enrolment \\
\hline Jain 2017 & Inappropriate reference standard \\
\hline Jing 2017 & Includes both adults and children or no information about age of enrolment \\
\hline Jipa 2017 & Could not extract $2 \times 2$ values \\
\hline Jorstad 2018 & Inappropriate reference standard \\
\hline Joythi 2019 & Children \\
\hline Kanade 2018 & Did not contain data by site for extrapulmonary TB \\
\hline
\end{tabular}




\begin{tabular}{|c|c|}
\hline Study & Reason for exclusion \\
\hline Kashyap 2019 & Inappropriate reference standard \\
\hline Kendall 2019 & Case-control study \\
\hline Kerkhoff 2017 & Did not contain specimen for extrapulmonary TB \\
\hline Khadka 2019 & Test other than Xpert MTB/RIF and Xpert Ultra \\
\hline Khan 2018 & Includes both adults and children or no information about age of enrolment \\
\hline Kilfoil 2015 & Could not extract $2 \times 2$ values \\
\hline Kim 2014 & $\begin{array}{l}\text { Could not extract } 2 \times 2 \text { values; unclear if culture-positive; pleural fluid (3), CSF (2); peritoneal fluid } \\
\text { (1) }\end{array}$ \\
\hline Kim 2015b & Case-control study \\
\hline Kim 2015c & Could not extract $2 \times 2$ values \\
\hline Kotovich 2018 & Inappropriate reference standard \\
\hline Koul 2018 & Could not extract $2 \times 2$ values \\
\hline Kumar 2017 & Case-control study \\
\hline Kumari 2019 & Test other than Xpert MTB/RIF and Xpert Ultra \\
\hline Kurbaniyazova 2017 & Did not contain specimen for extrapulmonary TB \\
\hline Kwak 2015 & Duplicate data \\
\hline Lawn 2012 & Screening study \\
\hline Lawn 2013 & Could not extract $2 \times 2$ values \\
\hline Lawn 2015 & Screening study \\
\hline Lawn 2017 & Could not extract $2 \times 2$ values \\
\hline Lee 2017 & Duplicate data \\
\hline Lemus-Minor 2018 & Did not contain specimen for extrapulmonary TB \\
\hline Li 2018 & Inappropriate reference standard \\
\hline Li 2020 & Test other than Xpert MTB/RIF and Xpert Ultra \\
\hline Liu 2015 & Duplicate data \\
\hline Liu 2019 & Did not contain specimen for extrapulmonary TB \\
\hline Lombardi 2017 & Could not extract data by site of extrapulmonary TB \\
\hline Marouane 2014 & Abstract; we excluded the publication, Marouane 2016, because we could not extract $2 \times 2$ values \\
\hline
\end{tabular}




\begin{tabular}{|c|c|}
\hline Study & Reason for exclusion \\
\hline Marouane 2016 & Could not extract $2 \times 2$ values \\
\hline Massi 2017 & Includes both adults and children or no information about age of enrolment \\
\hline Mathew 2018 & Did not contain data by site for extrapulmonary TB \\
\hline Mazzola 2016 & Includes both adults and children or no information about age of enrolment \\
\hline McMillen 2018 & Did not contain data by site for extrapulmonary TB \\
\hline Mechal 2019 & Test other than Xpert MTB/RIF and Xpert Ultra \\
\hline Metaferia 2018 & Could not extract $2 \times 2$ values \\
\hline Miller 2011 & $\begin{array}{l}\text { Fewer than } 5 \text { specimens for a given type of specimen; lymph node biopsy ( } 3 \text { specimens, of which } 1 \\
\text { was culture-positive) and endometrial biopsy ( } 1 \text { specimen that was culture-positive) }\end{array}$ \\
\hline Mishra 2017 & Abstract; we did not identify a published study \\
\hline Moure 2011 & $\begin{array}{l}\text { Fewer than } 5 \text { specimens for a given type of specimen: CSF ( } 3 \text { specimens, all culture-negative); } \\
\text { pleural fluid ( } 4 \text { specimens, } 2 \text { culture-positive); lymph node aspirate ( } 1 \text { specimen, culture-negative); } \\
\text { urine ( } 2 \text { specimens, both culture-positive); peritoneal fluid ( } 2 \text {, both culture-negative) }\end{array}$ \\
\hline Moure 2012 & Case-control study \\
\hline Negi 2019 & Case-control study \\
\hline Nhu 2013 & Inappropriate reference standard \\
\hline Omar 2019 & Test other than Xpert MTB/RIF and Xpert Ultra \\
\hline Pandey 2017 & Includes both adults and children or no information about age of enrolment \\
\hline Paramitha 2018 & Could not extract $2 \times 2$ values \\
\hline Park 2019 & Inappropriate reference standard \\
\hline Patel 2014 & Duplicate data \\
\hline Patel 2020 & Test other than Xpert MTB/RIF and Xpert Ultra \\
\hline Peter 2012 & Case-control study \\
\hline Philip 2017 & Inappropriate reference standard \\
\hline Piersimoni 2019 & Could not extract $2 \times 2$ tables \\
\hline Pink 2016 & Includes both adults and children or no information about age of enrolment \\
\hline Pohl 2016 & Children \\
\hline Porcel 2013 & Case-control study \\
\hline Rachow 2012 & Did not contain specimen for extrapulmonary TB \\
\hline
\end{tabular}




\begin{tabular}{|c|c|}
\hline Study & Reason for exclusion \\
\hline Raizada 2015 & Inappropriate reference standard \\
\hline Raizada 2018 & Children \\
\hline Ramamurthy 2016 & Could not extract data by site of extrapulmonary TB \\
\hline Rathour 2019 & Children \\
\hline Razack 2014 & Test other than Xpert MTB/RIF and Xpert Ultra \\
\hline Rebecca 2018 & Children \\
\hline Reddy 2017 & Could not extract $2 \times 2$ values \\
\hline Rindi 2017 & Case-control study \\
\hline Rossato Silva 2018 & Did not contain specimen for extrapulmonary TB \\
\hline Ruiz 2017 & Did not contain data by site for extrapulmonary TB \\
\hline Sachdeva 2018 & Includes both adults and children or no information about age of enrolment \\
\hline Saeed 2017a & Includes both adults and children or no information about age of enrolment \\
\hline Saeed 2017b & Could not extract $2 \times 2$ values \\
\hline Saeed 2018 & Did not contain data by site for extrapulmonary TB \\
\hline Salvador 2015 & Case-control study \\
\hline Samuel 2018 & Inappropriate reference standard \\
\hline Sanjuan Jimenez 2015 & Case-control study \\
\hline Schutz 2019 & Test other than Xpert MTB/RIF and Xpert Ultra \\
\hline Sekyere 2019 & Could not extract $2 \times 2$ data \\
\hline Set 2018 & Did not contain data by site for extrapulmonary TB \\
\hline Set 2019 & Test other than Xpert MTB/RIF and Xpert Ultra \\
\hline Shah 2016a & Case-control study \\
\hline Shakeel 2018 & Did not contain data by site for extrapulmonary TB \\
\hline Sharma 2017a & Did not contain data by site for extrapulmonary TB \\
\hline Sharma 2019 & Did not include specimen of interest \\
\hline Singanayagam 2014 & Could not extract $2 \times 2$ values \\
\hline Singh 2016 & Could not extract $2 \times 2$ values \\
\hline Smith 2014 & Did not contain specimen for extrapulmonary TB \\
\hline
\end{tabular}




\begin{tabular}{|c|c|}
\hline Study & Reason for exclusion \\
\hline Solomons 2015 & Duplicate data \\
\hline Solomons 2016 & Includes both adults and children or no information about age of enrolment \\
\hline Soomro 2017 & Could not extract $2 \times 2$ values \\
\hline Sumayya 2019 & Test other than Xpert MTB/RIF and Xpert Ultra \\
\hline Tahseen 2019 & Test other than Xpert MTB/RIF and Xpert Ultra \\
\hline Talib 2019 & Test other than Xpert MTB/RIF and Xpert Ultra \\
\hline Tang 2017 & Could not extract $2 \times 2$ values \\
\hline Tang 2018 & Case-control study \\
\hline Teo 2011 & Includes both adults and children or no information about age of enrolment \\
\hline Terzi 2019 & Could not obtain; same publication as Aydemir 2019 \\
\hline Theron 2014b & Duplicate data \\
\hline Tortoli 2012 & Includes both adults and children or no information about age of enrolment \\
\hline Toure 2017 & Could not extract $2 \times 2$ values \\
\hline Uddin 2019 & Test other than Xpert MTB/RIF and Xpert Ultra \\
\hline Vallejo 2015 & Could not extract $2 \times 2$ values \\
\hline Verghese 2016 & Abstract; we did not identify a published study \\
\hline Wang 2016a & Could not extract $2 \times 2$ values \\
\hline Wang 2016b & Includes both adults and children or no information about age of enrolment \\
\hline Wang 2018 & Inadequate reference standard \\
\hline Wei 2016 & Inappropriate reference standard \\
\hline Yang 2017 & Could not obtain $2 \times 2$ values \\
\hline Yang 2019 & Test other than Xpert MTB/RIF and Xpert Ultra \\
\hline Yu 2020 & Test other than Xpert MTB/RIF and Xpert Ultra \\
\hline Yuan 2016 & Inappropriate reference standard \\
\hline Zhang 2016 & Could not extract $2 \times 2$ values \\
\hline Zhou 2020 & Test other than Xpert MTB/RIF and Xpert Ultra \\
\hline Zurcher 2019 & Test other than Xpert MTB/RIF and Xpert Ultra \\
\hline
\end{tabular}

TB: tuberculosis. 
ADDITIONAL TABLES

Table 1. Forms of extrapulmonary TB

\section{Form of extrapul- Characteristics monary TB}

Tuberculous meningitis

\section{Diagnostic speci- mens and means of collection}

Cerebrospinal fluid, acquired by lumbar puncture with or without radiological guidance; biopsy of tuberculoma, acquired surgically infection that occurs before tuberculous meningitis develops), change of consciousness at presentation, bulging anterior fontanel, and seizures (Thwaites 2013). Tuberculous meningitis is sometimes associated with a concurrent cerebral tuberculoma, or, more rarely, a tuberculous abscess

Pleural tuberculosis, also called TB pleurisy
TB infection of the pleura presents with gradual onset of pleuritic chest pain, shortness of breath, fever, night sweats, and weight loss. Chest X-ray may demonstrate unilateral or occasionally bilateral pleural effusion. The severity of symptoms is highly variable, with many patients experiencing spontaneous resolution of symptoms, while others may develop severe pleural effusions requiring drainage. Pleuro-pulmonary tuberculosis, in which parenchymal lung involvement is visible on a chest X-ray, is associated with higher mortality than isolated pleural infection, which appears to be rarely fatal (Shu 2011)
Pleural fluid; pleural biopsy, which may be performed via thoracoscopy or percutaneously with Abram's needle, with or without ultrasound guidance

Fine-needle aspiration of fluid from affected lymph node, with or without radiological guidance; surgical biopsy of superficial lymph nodes; endoscopic biopsy of deep lymph nodes with ultrasound guidance
Bone or joint tuberculosis
Tuberculosis of the lymph nodes may affect one node or a group of nodes, or multiple groups within a chain. Lymph node tuberculosis is relatively more common among children than adults. The most common presentation is of a single, firm, non-tender enlarged node in the neck, although any lymph node group can be affected. This may be accompanied by fever, weight loss, and night sweats, particularly in people with HIV. Patients with tuberculosis in deep lymph nodes, such as the mediastinal or mesenteric lymph nodes, may present with fever, night sweats, and weight loss, or, more rarely, with symptoms related to compression of adjacent structures. Over time lymph nodes become fluctuant and may discharge via a sinus to the skin or an adjacent viscus. It should be noted that lymphadenopathy may also be seen in other forms of tuberculosis as part of the immune response, but this is not usually caused by direct infection of the lymph nodes
Tuberculosis of bones or joints or both causes chronic pain, deformity, and disability, and tuberculosis of the cervical spine can be life-threatening. The usual presenting symptom is pain. Fever and weight loss, with or without signs of spinal cord compression, may be present. Patients with advanced disease may have severe pain, spinal deformity, paraspinal muscle wasting, and neurological deficit. Children may have failure to thrive and difficulty walking
Aspiration of joint fluid or periarticular abscesses; percutaneous computed tomography-guided biopsy of lesions is preferred, but some patients may require open biopsy

Urine; biopsy of affected organs, acquired under radiological guidance or surgically
Genitourinary tuberculosis
Tuberculosis of the genitourinary tract includes renal tuberculosis and tuberculosis of the reproductive system. Renal tuberculosis presents with flank pain, haematuria, and dysuria. Female genital tuberculosis presents with infertility (and may be otherwise asymptomatic), pelvic pain, and vaginal bleeding. Testicular tuberculosis presents with a scrotal mass and infertility 


\section{Table 1. Forms of extrapulmonary TB (Continued)}

Pericardial tubercu- Tuberculosis of the pericardium presents with fever, malaise, night sweats, and losis, also called TB pericarditis weight loss. Chest pain and shortness of breath are also commonly-experienced symptoms. Pericardial tuberculosis may be associated with pericardial effusion, which can be severe and lead to life-threatening tamponade. Some patients go on to develop pericardial constriction, which can lead to heart failure and death and may require surgical intervention even after mycobacterial cure
Pericardial fluid acquired by pericardiocentesis; pericardial biopsy, acquired under radiological guidance or surgically
Peritoneal tuberculosis
Tuberculosis of the peritoneum usually presents with pain and abdominal swelling, which may be accompanied by fever, weight loss, and anorexia
Ascitic fluid acquired by paracentesis; peritoneal biopsy (Chow 2002)
Disseminated tuberculosis, also called miliary tuberculosis. It has been proposed that the designation 'miliary TB' be restricted to disseminated TB with miliary shadows on chest radiograph (Reuter 2009)
Disseminated tuberculosis involves two or more distinctly separate sites. Manifestations may be varied, ranging from acute fulminant disease to non-specific symptoms of fever, weight loss, and weakness. HIV-positive people are more likely to have disseminated tuberculosis than HIV-negative people. In a systematic review of the prevalence of tuberculosis in post mortem evaluations of HIV-positive people, among adults disseminated tuberculosis was found in $88 \%$ of tuberculosis cases and was considered the cause of death in $91 \%$ of TB cases (Gupta 2015)
Blood; specimens acquired from affected extrapulmonary sites

Abbreviations: TB: tuberculosis.

We adapted the table from Sharma 2017b. 


\begin{tabular}{|c|c|c|c|c|c|c|c|c|}
\hline $\begin{array}{l}\text { Type of } \\
\text { specimen }\end{array}$ & Test & $\begin{array}{l}\text { Reference } \\
\text { standard }\end{array}$ & $\begin{array}{l}\text { Number } \\
\text { studies } \\
\text { (partici- } \\
\text { pants) }\end{array}$ & $\begin{array}{l}\text { Number } \\
\text { (\%) with } \\
\text { TB or ri- } \\
\text { fampicin } \\
\text { resistance }\end{array}$ & $\begin{array}{l}\text { Pooled sensitivity } \\
\text { (95\% Crl) }\end{array}$ & $\begin{array}{l}\text { Pooled specificity } \\
\text { (95\% Crl) }\end{array}$ & $\begin{array}{l}\text { Positive predictive } \\
\text { value }(95 \% \text { Crl) }\end{array}$ & $\begin{array}{l}\text { Negative predictive } \\
\text { value }(95 \% \mathrm{Crl})\end{array}$ \\
\hline CSF & Xpert Ultra & culture & $6(475)$ & $89(18.7)$ & $89.4 \%$ (79.1 to 95.6$)$ & $91.2 \%$ (83.2 to 95.7$)$ & $53.0 \%$ (36.6 to 69.6 ) & $98.7 \%$ (97.5 to 99.5$)$ \\
\hline CSF & Xpert Ultra & composite & $4(496)$ & $160(32.2)$ & $62.7 \%$ (45.7 to 77.0 ) & $99.1 \%$ (96.6 to 99.9$)$ & $87.9 \%(65.5$ to 99.0$)$ & $96.0 \%$ (94.2 to 97.5$)$ \\
\hline CSF & $\begin{array}{l}\text { Xpert MTB/ } \\
\text { RIF }\end{array}$ & culture & $30(3395)$ & $571(16.8)$ & $71.1 \%(62.8$ to 79.1$)$ & $96.9 \%$ (95.4 to 98.0$)$ & $71.8 \%$ (62.3 to 80.7$)$ & $96.8 \%$ (95.9 to 97.7$)$ \\
\hline CSF & $\begin{array}{l}\text { Xpert MTB/ } \\
\text { RIF }\end{array}$ & composite & $14(2203)$ & $862(39.1)$ & $42.3 \%$ (32.1 to 52.8$)$ & $99.8 \%(99.3$ to 100.0$)$ & $96.3 \%$ (87.2 to 100.0$)$ & $94.0 \%(93.0$ to 95.0$)$ \\
\hline CSF & $\begin{array}{l}\text { Ultra, direct } \\
\text { comparison }\end{array}$ & culture & $5(471)$ & $86(18.3)$ & $89.0 \%$ (77.9 to 95.2$)$ & $91.0 \%$ (82.7 to 95.6$)$ & $52.2 \%$ (35.6 to 69.0$)$ & $98.7 \%$ (97.3 to 99.4$)$ \\
\hline CSF & $\begin{array}{l}\text { Xpert MTB/ } \\
\text { RIF, direct } \\
\text { comparison }\end{array}$ & culture & $5(471)$ & $87(18.5)$ & $62.2 \%(43.7$ to 78.1$)$ & $96.8 \%$ (93.4 to 98.6$)$ & $68.4 \%$ (49.0 to 83.6$)$ & $95.8 \%$ (93.9 to 97.5$)$ \\
\hline $\begin{array}{l}\text { Pleural flu- } \\
\text { id }\end{array}$ & Xpert Ultra & culture & $4(398)$ & $158(39.7)$ & $75.0 \%$ (58.0 to 86.4 ) & $87.0 \%$ (63.1 to 97.9$)$ & $38.8 \%$ (17.9 to 79.5$)$ & $96.9 \%(94.5$ to 98.3$)$ \\
\hline $\begin{array}{l}\text { Pleural flu- } \\
\text { id }\end{array}$ & $\begin{array}{l}\text { Xpert MTB/ } \\
\text { RIF }\end{array}$ & culture & $25(3065)$ & $644(21.0)$ & $49.5 \%$ (39.8 to 59.9$)$ & $98.9 \%$ (97.6 to 99.7 ) & $83.2 \%$ (68.9 to 94.6$)$ & $94.6 \%(93.7$ to 95.7$)$ \\
\hline $\begin{array}{l}\text { Pleural flu- } \\
\text { id }\end{array}$ & $\begin{array}{l}\text { Xpert MTB/ } \\
\text { RIF }\end{array}$ & composite & $10(1024)$ & $616(60.1)$ & $\begin{array}{l}18.9 \% \\
(11.5 \text { to } 27.9)\end{array}$ & $\begin{array}{l}99.3 \% \\
\text { (98.1 to } 99.8 \text { ) }\end{array}$ & $73.6 \%$ (49.2 to 91.2 ) & $91.7 \%$ (91.0 to 92.5$)$ \\
\hline $\begin{array}{l}\text { Lymph } \\
\text { node aspi- } \\
\text { rate }\end{array}$ & $\begin{array}{l}\text { Xpert MTB/ } \\
\text { RIF }\end{array}$ & culture & $14(1588)$ & $627(39.5)$ & $88.9 \%$ (82.7 to 93.6$)$ & $86.2 \%$ (78.0 to 92.3$)$ & $41.7 \%$ (31.4 to 55.5$)$ & $98.6 \%$ (97.9 to 99.2$)$ \\
\hline $\begin{array}{l}\text { Lymph } \\
\text { node aspi- } \\
\text { rate }\end{array}$ & $\begin{array}{l}\text { Xpert MTB/ } \\
\text { RIF }\end{array}$ & composite & $4(679)$ & $377(55.5)$ & $\begin{array}{l}81.6 \% \\
\text { (61.9 to } 93.3 \text { ) }\end{array}$ & $\begin{array}{l}96.4 \% \\
\text { (91.3 to } 98.6)\end{array}$ & $71.0 \%$ (51.1 to 86.1$)$ & $97.9 \%$ (95.8 to 99.2 ) \\
\hline
\end{tabular}




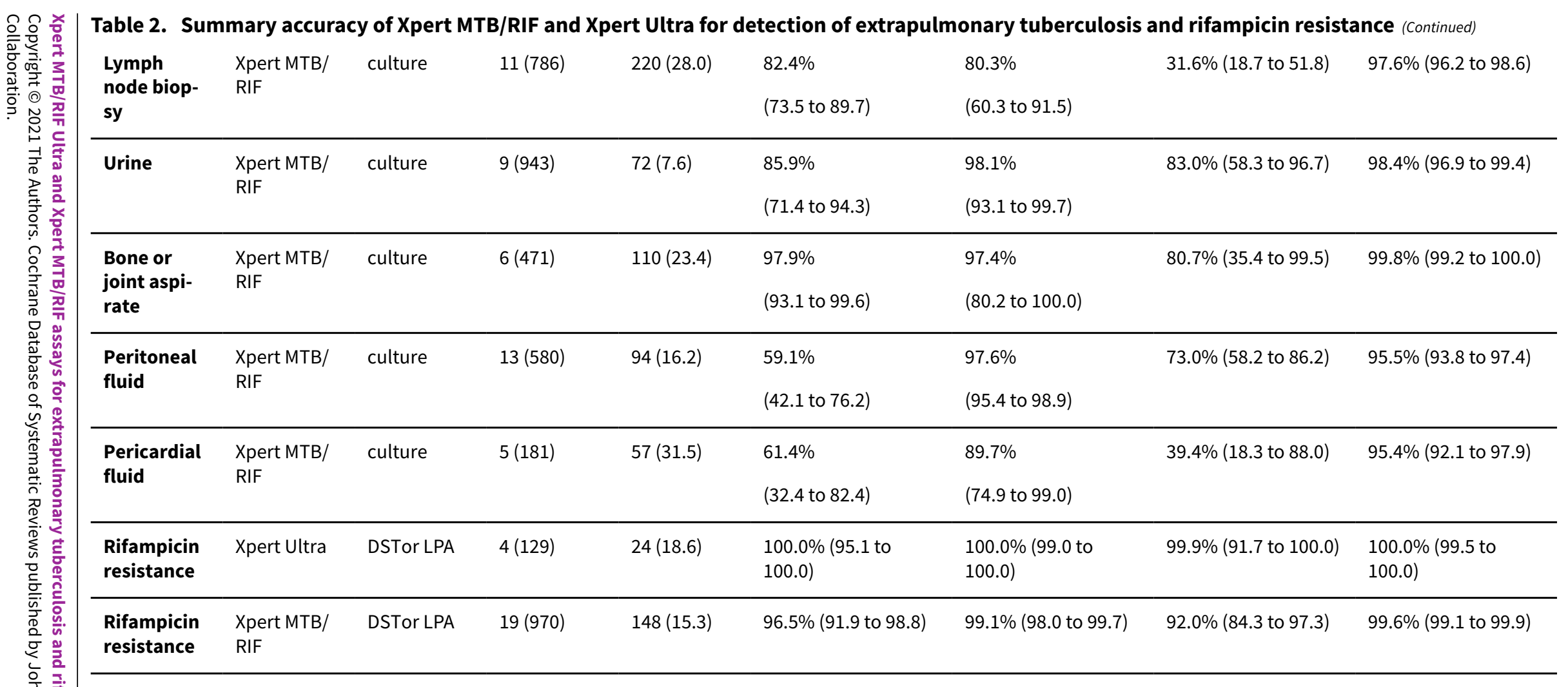

Abbreviations: Crl: credible interval; CSF: cerebrospinal fluid; LPA: Line probe assay; TB: tuberculosis.

Studies included in the table are limited to those that report data for both sensitivity and specificity; thus the number of studies (specimens) may differ slightly from those reported in the main text of the review. For tuberculosis detection, the reference standard was culture and a composite reference standard. For rifampicin resistance detection, the reference standards were culture-based drug susceptibility testing or line probe assay. Pooled sensitivity and pooled specificity are posterior median estimates. 
Table 3. Latent class meta-analysis

\begin{tabular}{|c|c|c|c|c|c|c|}
\hline $\begin{array}{l}\text { Form of extrapulmonary tu- } \\
\text { berculosis, type of specimen }\end{array}$ & $\begin{array}{l}\text { Num- } \\
\text { ber of } \\
\text { studies } \\
\text { (par- } \\
\text { tici- } \\
\text { pants) }\end{array}$ & $\begin{array}{l}\text { Cul- } \\
\text { ture-con- } \\
\text { firmed } \\
\text { tubercu- } \\
\text { losis }(\%)\end{array}$ & $\begin{array}{l}\text { Pooled sensi- } \\
\text { tivity ( } 95 \% \mathrm{Crl})\end{array}$ & $\begin{array}{l}\text { Pooled speci- } \\
\text { ficity }(95 \% \mathrm{Crl})\end{array}$ & $\begin{array}{l}\text { Positive predic- } \\
\text { tive value }(95 \% \\
\text { Crl) }\end{array}$ & $\begin{array}{l}\text { Negative pre- } \\
\text { dictive value } \\
\text { (95\% Crl) }\end{array}$ \\
\hline \multicolumn{7}{|c|}{ Accuracy estimates of Xpert MTB/RIF } \\
\hline $\begin{array}{l}\text { Tuberculous meningitis, } \\
\text { cerebrospinal fluid }\end{array}$ & $\begin{array}{l}30 \\
(3395)\end{array}$ & $\begin{array}{l}571 \\
(16.8)\end{array}$ & $\begin{array}{l}74.7 \%(65.5 \text { to } \\
84.0)\end{array}$ & $\begin{array}{l}99.5 \%(99.1 \text { to } \\
99.7)\end{array}$ & $\begin{array}{l}94.5 \%(89.7 \text { to } \\
96.9)\end{array}$ & $\begin{array}{l}97.3 \%(96.3 \text { to } \\
98.3)\end{array}$ \\
\hline Pleural tuberculosis, fluid & $\begin{array}{l}25 \\
(3065)\end{array}$ & $\begin{array}{l}644 \\
(21.0)\end{array}$ & $\begin{array}{l}53.1 \%(42.8 \text { to } \\
64.1)\end{array}$ & $\begin{array}{l}99.6 \%(99.3 \text { to } \\
99.8)\end{array}$ & $\begin{array}{l}93.7 \%(89.5 \text { to } \\
96.5)\end{array}$ & $\begin{array}{l}95.0 \%(94.0 \text { to } \\
96.2)\end{array}$ \\
\hline $\begin{array}{l}\text { Lymph node tuberculosis, as- } \\
\text { pirate }\end{array}$ & $\begin{array}{l}14 \\
(1588)\end{array}$ & $\begin{array}{l}627 \\
(39.5)\end{array}$ & $\begin{array}{l}91.5 \%(85.2 \text { to } \\
95.9)\end{array}$ & $\begin{array}{l}99.5 \% \\
\text { (99.1 to } 99.7)\end{array}$ & $\begin{array}{l}95.2 \%(91.4 \text { to } \\
97.5)\end{array}$ & $\begin{array}{l}99.1 \%(98.4 \text { to } \\
99.5)\end{array}$ \\
\hline \multicolumn{7}{|l|}{ Accuracy estimates of culture } \\
\hline $\begin{array}{l}\text { Tuberculous meningitis, } \\
\text { cerebrospinal fluid }\end{array}$ & $\begin{array}{l}30 \\
(3395)\end{array}$ & $\begin{array}{l}571 \\
(16.8)\end{array}$ & $\begin{array}{l}80.8 \%(72.5 \text { to } \\
88.5)\end{array}$ & $\begin{array}{l}99.2 \%(98.7 \text { to } \\
99.5)\end{array}$ & $\begin{array}{l}91.9 \%(86.9 \text { to } \\
95.1)\end{array}$ & $\begin{array}{l}97.9 \%(97.0 \text { to } \\
98.7)\end{array}$ \\
\hline Pleural tuberculosis, fluid & $\begin{array}{l}25 \\
(3065)\end{array}$ & $\begin{array}{l}644 \\
(21.0)\end{array}$ & $\begin{array}{l}89.5 \%(80.5 \text { to } \\
96.3)\end{array}$ & $\begin{array}{l}99.0 \%(98.2 \text { to } \\
99.5)\end{array}$ & $\begin{array}{l}90.8 \%(84.2 \text { to } \\
94.7)\end{array}$ & $\begin{array}{l}98.8 \%(97.9 \text { to } \\
99.6)\end{array}$ \\
\hline $\begin{array}{l}\text { Lymph node tuberculosis, as- } \\
\text { pirate }\end{array}$ & $\begin{array}{l}14 \\
(1588)\end{array}$ & $\begin{array}{l}627 \\
(39.5)\end{array}$ & $\begin{array}{l}82.9 \%(69.9 \text { to } \\
91.8)\end{array}$ & $\begin{array}{l}98.8 \%(97.8 \text { to } \\
99.4)\end{array}$ & $\begin{array}{l}88.7 \%(80.1 \text { to } \\
94.0)\end{array}$ & $\begin{array}{l}98.1 \%(96.7 \text { to } \\
99.1)\end{array}$ \\
\hline
\end{tabular}

Abbreviations: Crl: credible interval.

We generally used non-informative priors in the latent class model.

Accuracy estimates were determined using a bivariate random-effects approach for comparison.

Table 4. Accuracy of Xpert Ultra versus Xpert MTB/RIF in cerebrospinal fluid

\begin{tabular}{|c|c|c|c|c|}
\hline \multicolumn{5}{|c|}{ Detection of tuberculosis in CSF } \\
\hline $\begin{array}{l}\text { Test (studies, } \\
\text { participants) }\end{array}$ & Xpert Ultra $(5,471)$ & Xpert MTB/RIF $(5,471)$ & $\begin{array}{l}\text { Difference (Xpert Ultra minus } \\
\text { Xpert MTB/RIF) }\end{array}$ & $\begin{array}{l}\text { Probability (Xpert } \\
\text { Ultra minus Xpert } \\
\text { MTB/RIF) }\end{array}$ \\
\hline Sensitivity & $89.0 \%$ (77.9 to 95.2$)$ & $62.2 \%(43.7$ to 78.1$)$ & $26.2 \%(9.1$ to 44.4$)$ & 0.997 \\
\hline Specificity & $91.0 \%(82.7$ to 95.6$)$ & $96.8 \%$ (93.4 to 98.6 ) & $-5.6 \%(-12.9$ to -0.1$)$ & 0.022 \\
\hline
\end{tabular}

Table 5. Impact of concentrating cerebrospinal fluid on Xpert Ultra and Xpert MTB/RIF sensitivity and specificity

$\begin{array}{lll}\text { Covariate (number of studies, participants) } \quad \text { Pooled sensitivity (95\% Crl) } & \begin{array}{l}\text { Pooled specificity (95\% } \\ \text { Crl) }\end{array}\end{array}$

\section{Concentration step, Xpert Ultra}


Table 5. Impact of concentrating cerebrospinal fluid on Xpert Ultra and Xpert MTB/RIF sensitivity and

\begin{tabular}{|c|c|c|}
\hline $\begin{array}{l}\text { Zecificity (continued) } \\
\text { Zoncentrated specimen }(3,421)\end{array}$ & $90.5 \%(76.7$ to 97.0$)$ & $91.9 \%(84.5$ to 96.1$)$ \\
\hline Unconcentrated specimen $(3,54)$ & $88.4 \%(67.8$ to 97.5$)$ & $88.6 \%$ (58.4 to 99.0$)$ \\
\hline Difference (concentrated minus unconcentrated) & $2.6 \%(-13.9$ to 24.1$)$ & $3.4 \%(-9.5$ to 32.8$)$ \\
\hline Probability (concentrated minus unconcentrated) & 0.630 & 0.653 \\
\hline \multicolumn{3}{|l|}{ Concentration step, Xpert MTB/RIF } \\
\hline Concentrated specimen $(14,2279)$ & $77.6 \%(67.2$ to 85.9$)$ & $97.4 \%(96.1$ to 98.4$)$ \\
\hline Unconcentrated specimen $(17,1123)$ & $59.4 \%(48.3$ to 70.5$)$ & $96.8 \%(94.0$ to 98.7$)$ \\
\hline Difference (concentrated minus unconcentrated) & $18.4 \%(2.8$ to 32.1$)$ & $0.6 \%(-1.7$ to 3.6$)$ \\
\hline Probability (concentrated minus unconcentrated) & 0.989 & 0.696 \\
\hline
\end{tabular}

Abbreviations: Crl: credible interval.

Table 6. Impact of tuberculosis prevalence on Xpert Ultra and Xpert MTB/RIF sensitivity and specificity

$\begin{array}{lll}\text { Analysis, (number of studies, specimens) } & \begin{array}{l}\text { Pooled sensitivity (95\% } \\ \text { Crl) }\end{array} & \begin{array}{l}\text { Pooled specificity (95\% } \\ \text { Crl) }\end{array}\end{array}$
Pooled sensitivity (95\% $\quad$ Pooled
Crl)

\begin{tabular}{|c|c|c|}
\hline \multicolumn{3}{|l|}{ Cerebrospinal fluid, Xpert Ultra } \\
\hline Among studies with prevalence $\geq 30 \%(3,54)$ & $88.3 \%$ (68.3 to 97.0$)$ & $88.0 \%$ (64.3 to 97.9$)$ \\
\hline Among studies with prevalence $<30 \%(3,421)$ & $90.8 \%$ (77.3 to 96.9$)$ & $91.9 \%(82.5$ to 96.6$)$ \\
\hline Difference ( $\geq \mathbf{3 0} \%$ group minus $<30 \%$ group) & $-2.2 \%(-23.0$ to 13.5$)$ & $-3.8 \%(-27.7$ to 9.8$)$ \\
\hline Probability ( $\geq 30 \%$ group minus $<30 \%$ group) & 0.390 & 0.308 \\
\hline \multicolumn{3}{|l|}{ Cerebrospinal fluid, Xpert MTB/RIF } \\
\hline Among studies with prevalence $\geq 30 \%(6,610)$ & $67.0 \%(49.0$ to 81.5$)$ & $94.1 \%(86.8$ to 97.9$)$ \\
\hline Among studies with prevalence $<30 \%(24,2785)$ & $72.0 \%(62.4$ to 81.2$)$ & $97.3 \%$ (95.9 to 98.3$)$ \\
\hline Difference ( $\geq \mathbf{3 0} \%$ group minus $<30 \%$ group) & $-4.8 \%(-25.5$ to 12.1$)$ & $-3.1 \%(-10.5$ to 0.8$)$ \\
\hline Probability ( $\geq 30 \%$ group minus $<30 \%$ group) & 0.296 & 0.071 \\
\hline \multicolumn{3}{|l|}{ Cerebrospinal fluid, Xpert MTB/RIF } \\
\hline Among studies with prevalence $\geq 10 \%(19,2190)$ & $68.7 \%(58.5$ to 78.0$)$ & $95.1 \%(92.7$ to 96.8$)$ \\
\hline Among studies with prevalence $<10 \%(11,1205)$ & $74.2 \%(57.4$ to 86.6$)$ & $98.6 \%$ (97.5 to 99.3$)$ \\
\hline Difference ( $\geq 10 \%$ group minus $<10 \%$ group) & $-5.5 \%(-21.2$ to 13.3$)$ & $-3.5 \%(-6.0$ to -1.5$)$ \\
\hline Probability ( $\geq 10 \%$ group minus $<10 \%$ group) & 0.272 & 0.001 \\
\hline
\end{tabular}


Table 6. Impact of tuberculosis prevalence on Xpert Ultra and Xpert MTB/RIF sensitivity and specificity (Continued) Pleural fluid, Xpert MTB/RIF

\begin{tabular}{|c|c|c|}
\hline Among studies with prevalence $\geq 50 \%(6,627)$ & $20.7 \%(11.2$ to 33.7$)$ & $99.6 \%$ (97.9 to 99.9$)$ \\
\hline Among studies with prevalence $<50 \%(4,397)$ & $15.5 \%(6.5$ to 30.1$)$ & $99.0 \%$ (96.9 to 99.8$)$ \\
\hline Difference ( $\geq 50 \%$ group minus $<50 \%$ group) & $5.1 \%(-11.8$ to 21.2$)$ & $0.5 \%(-1.2$ to 2.7$)$ \\
\hline Probability ( $\geq \mathbf{5 0} \%$ group minus $<\mathbf{5 0} \%$ group) & 0.757 & 0.759 \\
\hline \multicolumn{3}{|l|}{ Lymph node aspirate, Xpert MTB/RIF } \\
\hline Among studies with prevalence $\geq 35(9,911)$ & $93.1 \%$ (88.9 to 96.3$)$ & $83.2 \%$ (69.5 to 92.1$)$ \\
\hline Among studies with prevalence $<35 \%(5,677)$ & $72.2 \%$ (64.9 to 87.2$)$ & $90.0 \%$ (78.3 to 95.9$)$ \\
\hline Difference ( $\geq 35 \%$ group minus $<35 \%$ group) & $15.7 \%(5.4$ to 28.6$)$ & $-6.4 \%(-21.3$ to 76$)$ \\
\hline Probability ( $\geq 35 \%$ group minus $<35 \%$ group) & 0.999 & 0.158 \\
\hline
\end{tabular}

Abbreviations: Crl: credible interval.

Prevalence refers to the percentage of culture-confirmed tuberculosis specimens or confirmed rifampicin-resistant specimens in the study. We selected prevalence levels to approximate the lower bound of the interquartile range or in consideration of the range of prevalences reported in the included studies.

Table 7. Sensitivity analyses, Xpert Ultra in cerebrospinal fluid

\begin{tabular}{|c|c|c|c|c|c|}
\hline Type of specimen & $\begin{array}{l}\text { Num- } \\
\text { ber of } \\
\text { studies } \\
\text { (speci- } \\
\text { mens) }\end{array}$ & $\begin{array}{l}\text { Pooled sensitivity } \\
\text { (95\% Crl) }\end{array}$ & $\begin{array}{l}\text { Pooled specificity } \\
(95 \% \mathrm{Crl})\end{array}$ & $\begin{array}{l}\text { Predicted sensitivi- } \\
\text { ty }(95 \% \mathrm{Crl})\end{array}$ & $\begin{array}{l}\text { Predicted specifici- } \\
\text { ty }(95 \% \mathrm{Crl})\end{array}$ \\
\hline All participants & $6(475)$ & $89.4 \%$ (79.1 to 95.6$)$ & $91.2 \%(83.2$ to 95.7$)$ & $53.0 \%$ (36.6 to 69.6 ) & $98.7 \%$ (97.5 to 99.5$)$ \\
\hline $\begin{array}{l}\text { Consecutive partici- } \\
\text { pant selection }\end{array}$ & $5(471)$ & $87.9 \%$ (76.4 to 94.6$)$ & $90.4 \%(81.1$ to 95.1$)$ & $88.0 \%$ (65.2 to 96.7$)$ & $90.5 \%$ (65.5 to 97.7$)$ \\
\hline $\begin{array}{l}\text { Reference standard } \\
\text { blinding }\end{array}$ & $4(432)$ & $88.5 \%$ (74.7 to 95.6$)$ & $89.1 \%$ (76.9 to 94.3$)$ & $88.6 \%$ (63.4 to 97.2$)$ & $89.2 \%(61.0$ to 97.1$)$ \\
\hline $\begin{array}{l}\text { Single specimen per } \\
\text { participant }\end{array}$ & $4(432)$ & $88.5 \%$ (74.7 to 95.6$)$ & $89.1 \%$ (76.9 to 94.3$)$ & $88.6 \%$ (63.4 to 97.2$)$ & $89.2 \%(61.0$ to 97.1$)$ \\
\hline
\end{tabular}

Abbreviations: Crl: credible interval. 


\begin{tabular}{|c|c|c|c|c|c|c|c|}
\hline Systematic & Search period & Index test & & & Accuracy against cult & Ire reference standard & \\
\hline & & & $\begin{array}{l}\text { tal number } \\
\text { of extrapul- } \\
\text { monary spec- } \\
\text { imens) }\end{array}$ & of specimens & $\begin{array}{l}\text { Tuberculous menin- } \\
\text { gitis }\end{array}$ & $\begin{array}{l}\text { Pleural tuberculosis } \\
\text { (pleural fluid) }\end{array}$ & $\begin{array}{l}\text { Lymph node tuber- } \\
\text { culosis }\end{array}$ \\
\hline Chang $2012^{a}$ & $\begin{array}{l}\text { Up to } 1 \text { October } \\
2011\end{array}$ & Xpert MTB/RIF & 7 (1058) & $\begin{array}{l}\text { Multiple forms com- } \\
\text { bined }\end{array}$ & Not reported & Not reported & Not reported \\
\hline $\begin{array}{l}\text { Denkinger } \\
2014 \text { b }\end{array}$ & $\begin{array}{l}\text { Up to } 15 \\
\text { October } 2013\end{array}$ & Xpert MTB/RIF & $18(4461)$ & $\begin{array}{l}\text { Lymph node, pleural } \\
\text { fluid, CSF }\end{array}$ & $\begin{array}{l}\text { Sensitivity } 81 \% \text {; } \\
\text { specificity } 98 \%\end{array}$ & $\begin{array}{l}\text { Sensitivity } 46 \% \text {; speci- } \\
\text { ficity } 99 \%\end{array}$ & $\begin{array}{l}\text { Sensitivity } 83 \% \text {; } \\
\text { specificity } 94 \%\end{array}$ \\
\hline $\begin{array}{l}\text { May- } \\
\text { nard-Smith } \\
2014\end{array}$ & $\begin{array}{l}\text { Up to } 6 \text { Novem- } \\
\text { ber } 2013\end{array}$ & Xpert MTB/RIF & $27(6026)$ & $\begin{array}{l}\text { Lymph node, pleural } \\
\text { fluid, CSF, other forms }\end{array}$ & $\begin{array}{l}\text { Median sensitivi- } \\
\text { ty } 85 \% \text { (IQR } 75 \% \\
\text { to } 100 \%) \text {; median } \\
\text { specificity } 100 \% \text { (IQR } \\
98 \% \text { to } 100 \% \text { ) }\end{array}$ & $\begin{array}{l}\text { Sensitivity } 34 \% \text {; speci- } \\
\text { ficity } 98 \%\end{array}$ & $\begin{array}{l}\text { Sensitivity } 96 \% \\
\text { specificity } 93 \%\end{array}$ \\
\hline Penz 2015 & $\begin{array}{l}\text { Up to } 15 \text { August } \\
2014\end{array}$ & Xpert MTB/RIF & $36(9523)$ & $\begin{array}{l}\text { Lymph node, pleural } \\
\text { fluid, CSF, other forms }\end{array}$ & $\begin{array}{l}\text { Sensitivity } 69 \% \text {; } \\
\text { specificity } 97 \%\end{array}$ & $\begin{array}{l}\text { Sensitivity } 37 \% \text {; speci- } \\
\text { ficity } 98 \%\end{array}$ & $\begin{array}{l}\text { Sensitivity } 87 \% \text {; } \\
\text { specificity } 92 \%\end{array}$ \\
\hline Sehgal 2016 & $\begin{array}{l}\text { Up to } 31 \text { August } \\
2015\end{array}$ & Xpert MTB/RIF & $24(2486)$ & Pleural fluid & Not applicable & $\begin{array}{l}\text { Sensitivity 51\%; } \\
\text { specificity } 99 \%\end{array}$ & Not applicable \\
\hline Li Y 2017 c & $\begin{array}{l}\text { Up to } 20 \text { June } \\
2015\end{array}$ & Xpert MTB/RIF & $\begin{array}{l}26 \text { (not re- } \\
\text { ported) }\end{array}$ & $\begin{array}{l}\text { Multiple forms com- } \\
\text { bined }\end{array}$ & Not reported & Not reported & Not reported \\
\hline Gupta 2018 & $\begin{array}{l}\text { Up to } 25 \text { March } \\
2017\end{array}$ & Xpert MTB/RIF & $33(8977)$ & CSF & $\begin{array}{l}\text { Sensitivity } 57 \% \\
\text { specificity } 98 \%\end{array}$ & Not reported & Not reported \\
\hline $\begin{array}{l}\text { Pormoham- } \\
\text { mad } 2019\end{array}$ & Upto 11 Nov 2018 & Xpert MTB/RIF & $\begin{array}{l}16 \text { (not re- } \\
\text { ported) }\end{array}$ & $\mathrm{CSF}$ & $\begin{array}{l}\text { Sensitivity } 61 \% \text {; } \\
\text { specificity } 99 \%\end{array}$ & Not reported & Not reported \\
\hline Yu 2019 & Up to 6 July 2018 & Xpert MTB/RIF & $21(1629)$ & Lymph node & Not reported & Not reported & $\begin{array}{l}\text { Sensitivity } 84 \% \\
\text { specificity: } 91 \%\end{array}$ \\
\hline Zhang $2020 \mathrm{~d}$ & $\begin{array}{l}\text { Up to } 20 \text { May } \\
2019\end{array}$ & Xpert Ultra & $7(1500)$ & Multiple specimens & Not reported & Not reported & Not reported \\
\hline
\end{tabular}


${ }^{a}$ For all forms of extrapulmonary tuberculosis combined, Chang 2012 reported pooled sensitivity and specificity of $80.4 \%$ (95\% confidence interval (CI) 75.0 to 85.1$)$ and $86.1 \%$ $(95 \% \mathrm{Cl} 83.5$ to 88.4$)$, respectively.

bUsing a composite reference standard, Denkinger 2014 found the following pooled sensitivity and specificity estimates: lymph node tuberculosis (aspirate or tissue) $81.2 \%$ (95\%

$\mathrm{Cl} 72.4$ to 87.7$)$ and $99.1 \%(95 \% \mathrm{Cl} 94.5$ to 99.9$)$; pleural tuberculosis $21.4 \%(95 \% \mathrm{Cl} 8.8$ to 33.9 ) and $100 \%$ (95\% Cl 99.4 to 100$)$; and meningeal TB $62.8 \%$ (95\% $\mathrm{Cl} 47.7$ to 75.8 ) and $98.8 \%$ ( $95 \% \mathrm{Cl} 95.7$ to 100$)$, respectively.

cFor both pulmonary and extrapulmonary tuberculosis, review authors included 106 studies involving 52,410 specimens. For all forms of extrapulmonary tuberculosis combined, Li Y 2017 reported pooled sensitivity and specificity of 80\% (95\% Cl 69 to 88) and 97\% (95\% Cl 94 to 98), respectively.

d Zhang 2020 included 7 studies involving 1500 specimens. For all forms of extrapulmonary tuberculosis combined, pooled sensitivity and specificity were $85.1 \%$ (95\% $\mathrm{Cl} 76.7$ to $90.8 \%$ ) and $95.7 \%(95 \% \mathrm{Cl} 87.9$ to $98.6 \%)$, respectively.

Shen 2019 provided pooled estimates for bone or joint tuberculosis. The review included 14 studies with 1884 specimens with a pooled sensitivity of $96 \%$ and specificity of $85 \%$. 
Table 9. Prespecified changes for review update 2021

\begin{tabular}{ll}
\hline Protocol section & Appraisal points \\
\hline $\begin{array}{l}\text { Background and re- } \\
\text { search question }\end{array}$ & $\begin{array}{l}\text { - Review and update Back- } \\
\text { ground section, including } \\
\text { supporting references to } \\
\text { take account of any changes } \\
\text { that may have occurred. This } \\
\text { should include updating any } \\
\text { new information and current } \\
\text { policy debates on the topic }\end{array}$
\end{tabular}

\section{Address here}

This review update will describe the burden of extrapulmonary tuberculosis worldwide based on the latest WHO Global Tuberculosis Report. The Background will describe the updated WHO guidelines on molecular methods for diagnosing tuberculosis, including Xpert MTB/RIF and Xpert Ultra. The WHO Meeting to update the guidelines took place 3 - 6 December 2019. This Cochrane Review update will have informed these guidelines

This is a diagnostic test accuracy review.

Participants, index tests, and target condition, will be the same as in Kohli 2018 except as follows:

The update will be restricted to adults ( 15 years and older). The reason for this is that we have a separate Cochrane Review underway that is evaluating the tests for extrapulmonary tuberculosis in children.

We will add a composite reference standard, defined as culture or clinical criteria as defined by the primary study authors. The addition of a composite reference standard was specifically requested by the WHO and Guideline members.

The primary objectives are to assess the diagnostic accuracy of Xpert UItra for the diagnosis of extrapulmonary tuberculosis and to assess the diagnostic accuracy of Xpert Ultra for the detection of rifampicin resistance in adults.

Secondary objectives are the following.

- to investigate potential sources of heterogeneity in test accuracy, including volume of CSF for TB meningitis and processing methods for lymph node TB

- to compare the accuracy of Xpert Ultra and Xpert MTB/RIF in studies that evaluated both tests.

Concerning patient outcomes, the Discussion will summarize and refer to key findings in the test-treatment Cochrane Review by Haraka et al. (currently undergoing peer review). Although the Haraka review relates to pulmonary tuberculosis, it is the only evidence on patient outcomes of which we are aware.

We will use QUADAS-2 to appraise methodological quality of included studies consistent with Kohli 2018.

If data are sufficient, we will perform meta-analyses using a bivariate random-effects model. The analyses will include:

1. Xpert Ultra for different forms of extrapulmonary tuberculosis, culture reference standard

2. Xpert Ultra for different forms of extrapulmonary tuberculosis, composite reference standard

3. Accuracy of Xpert Ultra versus Xpert MTB/RIF in studies that evaluated both tests

4. Xpert Ultra for detecting rifampicin resistance 
Table 9. Prespecified changes for review update 2021 (Continued)

The different forms (and corresponding specimens for diagnosis) of extrapulmonary TB include: tuberculous meningitis, lymph node tuberculosis, pleural tuberculosis, genitourinary tuberculosis, bone or joint tuberculosis.

We will create 'Summary of findings' tables for the two primary objectives of the review.

TB: tuberculosis.

This table was approved by the CIDG editorial team on 17 December 2019.

WHAT'S NEW

\begin{tabular}{lll}
\hline Date & Event & Description \\
\hline 11 January 2021 & $\begin{array}{l}\text { New citation required but conclusions } \\
\text { have not changed }\end{array}$ & We updated the literature search and included 22 new studies. \\
\hline 11 January 2021 & New search has been performed & $\begin{array}{l}\text { We have updated the review with more information. There are } \\
\text { no major changes to the conclusions. }\end{array}$ \\
\hline
\end{tabular}

\section{HISTORY}

Protocol first published: Issue 8, 2017

Review first published: Issue 8, 2018

\section{CONTRIBUTIONS OFAUTHORS}

MK and KRS wrote early drafts of the protocol.

CMD and SGS contributed methodological advice.

KD contributed clinical expertise.

CMD and SGS tailored QUADAS-2 to the review.

MK and KRS reviewed the studies and extracted accuracy data.

MK and KRS assessed the methodological quality of included studies.

IS, MY, and ND performed the statistical analyses.

All review authors interpreted the findings.

MK, ND, and KRS wrote the first draft of the review.

MK and KRS prepared the 'Summary of findings' tables.

All review authors contributed to the final manuscript.

\section{DECLARATIONS OF INTEREST}

We have no financial involvement with any organization or entity that has a financial interest in, or financial conflict with, the subject matter or materials discussed in the review apart from those disclosed.

MK received funding from USAID, administered by the World Health Organization Global Tuberculosis Programme, Switzerland.

IS received funding from USAID, administered by the World Health Organization Global Tuberculosis Programme, Switzerland.

ND's participation in this project was supported in part by the Canadian Institutes of Health Research.

MY's participation in this project was supported in part by the Canadian Institutes of Health Research.

KD has no interests to declare.

CMD worked for the Foundation for Innovative New Diagnostics (FIND) until April 2019 and has no other interests to declare.

SGS works for FIND. FIND is a not-for-profit foundation whose mission is to find diagnostic solutions to overcome diseases of poverty in low- and middle-income countries. FIND works closely with the private and public sectors and receives funding from donors and some 
of its industry partners. FIND has an independent Scientific Advisory Committee and organizational firewalls that protect it against any undue influences in its work or in publication of its findings. More information on FIND's policy and guidelines for working with private sector partners can be found at www.finddx.org/ops-gov/.

KRS received funding from USAID, administered by the World Health Organization Global Tuberculosis Programme, Switzerland, and Cochrane infectious Diseases Group, UK. She has received financial support from McGill University, Canada, and the World Health Organization (WHO) Global Tuberculosis Programme, Switzerland for the preparation of systematic reviews and educational materials, consultancy fees from Foundation for Innovative New Diagnostics (FIND), Switzerland (for the preparation of systematic reviews and GRADE tables), honoraria, and travel support to attend WHO guideline meetings.

\section{SOURCES OF SUPPORT}

\section{Internal sources}

- Liverpool School of Tropical Medicine, UK

\section{External sources}

- Foreign, Commonwealth and Development Office (FCDO), UK

Project number 300342-104

- United States Agency for International Development (USAID), USA

Development of this project was in part made possible with financial support from USAID administered by the World Health Organization Global TB Programme

- Canadian Institutes of Health Research (CIHR) grant PJT-156039: Evaluating diagnostic accuracy of tests and decision rules in the absence of a perfect reference test: application to extrapulmonary tuberculosis, Canada

\section{DIFFERENCES BETWEEN PROTOCOLAND REVIEW}

Table 9 describes prespecified changes for this review update.

Selection criteria: We included studies where at least $85 \%$ of the participants enrolled were adults, aged 15 years or older, with presumptive extrapulmonary tuberculosis or rifampicin-resistant tuberculosis from all settings and countries. Restricting the age group to adults differs from the original review, where we also included children (Kohli 2018). We did this because children are now included in a separate Cochrane Review, Xpert MTB/RIF and Xpert MTB/RIF Ultra assays for active tuberculosis and rifampicin resistance in children (Kay 2020). We excluded studies where we could not disaggregate data on adults from those in children and studies where we could not tell the age of the participants enrolled.

QUADAS-2: we modified QUADAS-2 as follows.

Participant selection domain, applicability: For tuberculous meningitis, owing to the severity of the illness, we judged 'low concern' if participants were evaluated as inpatients at tertiary care centres. In the original review, we judged tertiary care to be a setting of high concern.

Reference standard domain: We clarified that CSF, pleural fluid, and lymph node aspirates are usually considered to be sterile, and standards specify that these specimens may be placed directly into the culture medium. Overly processing specimens may lead to falsenegative cultures. We scored 'yes' if studies did not use $\mathrm{N}$-acetyl-L-cysteine-sodium hydroxide for processing sterile specimens and 'unclear' if studies used $\mathrm{N}$-acetyl-L-cysteine-sodium hydroxide.

Investigations of heterogeneity: For specimen volume, we restricted this analysis to CSF because it was most clinically meaningful. For other fluid specimen types, the manufacturer's instructions for sputum were usually followed, requiring $2 \mathrm{~mL}$ of input fluid for the Xpert cartridge. In terms of the WHO standard operating procedure for lymph node tissue, we did not investigate this further because $80 \%(8 / 10)$ of the included studies followed the WHO recommendations. In performing the review, it became clear that because a homogenization step is part of the WHO standard operating procedure for preparing tissue specimens, there was no need to perform an additional separate analysis to confirm the presence of a homogenization step. We removed condition of specimen (fresh or frozen) from the analysis, because we identified only six studies in the current review that used frozen specimens, and we had already performed an analysis of this possible source of heterogeneity for the Cochrane Review on Xpert for pulmonary tuberculosis (Steingart 2014).

We have tried to eliminate stigmatizing language, for example, by changing 'suspected tuberculosis' to 'presumptive tuberculosis'.

For Xpert Ultra accuracy for lymph node tuberculosis, owing to insufficient data, we were unable to investigate processing methods for lymph node aspirate. 
GRADE: We elaborated on the means of applying GRADE to publication bias: We rated publication bias as undetected (not serious) for several reasons: the comprehensiveness of the literature search and extensive outreach to tuberculosis researchers to identify studies; the presence of only studies that produced precise estimates of high accuracy despite small sample size; and our knowledge about studies that were conducted but not published.

Unlike our previous review, in this update we did not extract information on manufacturers' involvement and funding. As both Xpert Ultra and Xpert MTB/RIF are available at a concessional price for researchers in resource-limited settings, and well-established tests, especially Xpert MTB/RIF, industry donation is rarely pursued. We acknowledge that, in addition to funding, there are other reasons for conflicts of interest, but we did not have time to pursue these. We are aware of a new tool being developed for this purpose: TACIT (Tool for Addressing Conflicts of Interest in Trials: tacit.one). We plan to avail ourselves of this new tool in future updates.

Sensitivity analyses: We stated in our protocol that for Xpert Ultra we would perform a sensitivity analysis by limiting studies to those that included only untreated participants. This information was often not reported in the publications and we did not contact primary study authors specifically about this question. We were therefore unable to confirm that studies met this criterion.

For the 'Summary of findings' tables, we prioritised culture as the reference standard (best reference standard for tuberculosis), apart from lymph node aspirate where we provide evidence using a composite reference standard, because, based on findings from the original review (Kohli 2018), we believe a composite reference is preferable for estimating accuracy.

We added post hoc a sensitivity analysis limiting inclusion to studies that used one specimen per participant.

\section{IN DEX TERMS}

\section{Medical Subject Headings (MeSH)}

Antibiotics, Antitubercular [ ${ }^{\star}$ therapeutic use]; Bacterial Proteins [ ${ }^{\star}$ genetics]; DNA-Directed RNA Polymerases [ ${ }^{\star}$ genetics]; Drug Resistance, Bacterial [ ${ }^{\star}$ genetics]; False Negative Reactions; False Positive Reactions; Mycobacterium tuberculosis [drug effects] [" genetics] [isolation \& purification]; *Reagent Kits, Diagnostic; Reference Standards; Rifampin [*therapeutic use]; Sensitivity and Specificity; Tuberculosis [cerebrospinal fluid] [ ${ }^{*}$ diagnosis] [drug therapy]; Tuberculosis, Meningeal [cerebrospinal fluid] [drug therapy]

\section{MeSH check words}

Humans 